\title{
Zur Geschichte des Vokalismus der ersten Silbe im Tscheremissischen und in den permischen Sprachen.
}

EINLEITUNG.

Die vorliegende Arbeit schliesst-sich als Fortsetzung an die früheren Untersuchungen des Verfassers an, die sich mit Fragen der finnisch-ugrischen Vokalgeschichte befassen ${ }^{1}$. und beruht auf Vorlesungen über finnisch-ugrische Vokallehre, die in den Jahren 1948-1951 gehalten worden sind.

Die Vokalismen des Tscheremissischen und der permischen Sprachen bilden kein geschlossenes Ganzes, sondern zwej getrennte Kapitel der finnisch-ugrischen Vokallehre. Chronologisch jedoch folgen diese Kapitel unmittelbar aufeinander und in dem Material von beiden kann der Forscher einige gleichgerichtete Tendenzen unterscheiden. Also ist ein gewisser Zusammenhang zwischen den beiden Teilen der Untersuchung vorhanden.

1 Themen derselben sind u.a. die Vokalkombinationen der finnischugrischen Sprachen und der Ursprung der finnisch-ugrischen Vokalharmonie (Virittäjä 1948, SS. 129-144), der urfinnische Vokalismus, insbesondere in einer Prüfung der Hypothese über die urfi. Mittelvokale (ibid. und Vir. 1945, SS. 158-182), die Geschichte des lappischen Vokalismus besonders auf Grund ostlappischen Materials (MSFOu. LXXIX), die Entwicklung des Vokalismus der ersten Silbe im Mordwinischen (FUF XXIX 288-336), chronologische Beobachtungen über den Schwund der Vokale der zweiten Silbe im Lrmordwinischen (Archiv für vergl. Phonetik V, SS. 147-148), die Entwicklung der Vokale der zweiten Silbe sowie des Akzentuationssystems im Urtscheremissischen (FUF XXIX 231-244). 
Bei der Klärung der Geschichte der Vokale der ersten Silbe im Lappischen und Mordwinischen hat der Verfasser zeigen wollen, dass man in beiden Sprachen von einem solchen system ausgehen muss, wie es im Frühurfinnischen bestanden hat und in den Hauptzügen unverändert in den meisten ostseefinnischen Sprachen erhalten geblieben ist. Diesmal wird dasselbe Erklärungsprinzip auf das tscheremissische und permische Material angewandt. Obwohl nach der Auffassung des Verfassers zwischen dem frühurfinnischen Vokalismus und dem der finnisch-ugrischen Ursprache in Wirklichkeit keine grundsätzlichen Unterschiede bestanden haben können, ist in dieser Untersuchung aus methodischen Gründen eine Zurückführung der Vokale der zu erforschenden Sprachen auf die finnisch-ugrischen Grundformen oder ein Vergleich mit diesen vermieden worden. Wenn als Vergleichsobjekt ein »ursprünglicher» Laut hingestellt worden ist, d.h. ein der frühurfinnischen Vertretung entsprechender, so wird beim Tscheremissischen lediglich von vortscheremissischer und bei den permischen Sprachen von vorpermischer Vertretung gesprochen. Genau genommen ist es ja erst dann berechtigt, Rekonstruktionen von fi.-ugr. Grundformen vorzulegen, wenn die Forschung auf alle Sprachen unserer Sprachfamilie ausgedehnt worden ist, auch auf die ugrische Gruppe, bei der jedoch die Herleitung der Vokalvertretungen von dem vorugrischen Sprachstand trotz der verdienstvollen Spezialuntersuchungen über die Vokalgeschichte dieser Sprachen noch ziemliche Schwierigkeiten bereitet. Diese Feststellung musste der Verfasser bei seinen obenerwähnten Vorlesungen machen. Die Klärung der Lautverhältnisse der wichtigen obugrischen Gruppe hat sich bisher hauptsächlich auf das ostjakische Material gestützt, das schon in allen Einzelheiten für die Forscher in reicher Fülle greifbar ist, aber die endgültige Synthese muss für so lange verschoben werden, bis das augenblicklich noch recht mangelhaft bekannte Wogulische in derselben Weise zugänglich ist. Irrig ist die Auffassung, dass das Ostjakische sich hinsichtlich des Vokalismus gegenüber dem Wogulischen in der Stellung einer archaischen Schlüsselsprache befinde; die zukünftige Forschung 
wird zweifellos auch vom Wogulischen her ausserordentlich bedeutungsvolle Ausblicke auf das Urobugrische eröffnen.

Unser Forschungsmaterial, das auf den SS. 156-185 veriffentlicht ist, strebt nach möglichster Ausführlichkeit. Lediglich die naturgemäss berechtigte Einschränkung ist gemacht worden, dass meist nur solches Wortmaterial aufgenommen worden ist, für das eine sichere oder wenigstens sehr wahrscheinliche etymologische Erklärung gegeben werden kann. Wenn Material verwendet worden ist, das als unsicher bezeichnet wird, zeigt ein Fragezeichen vor dem betreffenden Wort an, dass die für dieses aufgestellte Etymologie nach der Meinung des Verfassers doch richtig sein kann, während zwei Fragezeichen anzeigen, dass sie sehr zweifelhaft ist. Einige Etymologien, die zu der zweiten Gruppe gerechnet werden, in der früheren Literatur aber im allgemeinen vorbehaltlose Anerkennung finden, sind eigentlich nur dazu aufgenommen worden, um dem Verfasser Gelegenheit zu geben, seine kritische Einstellung zu vermerken. Bei der Gruppierung der Wortartikel ist die ursprüngliche Qualität der Vokale der ersten Silbe berücksichtigt worden, wobei in grossen Zügen die Reihenfolge $a, o, \bar{o}, u, \bar{u}, \ddot{a}, e, \bar{e}, i, \bar{\imath}, \ddot{u}$ eingehalten wird. Zwischenrubriken, die das Material streng einteilen würden, sind weggelassen worden, damit die ziemlich zahlreichen Wörter keine grundsätzlichen Einordnungsschwierigkeiten verursachen, bei denen man nicht mit unbedingter Sicherheit entscheiden kann, welcher von zwei einander nahestehenden Vokalen in der ersten Silbe gestanden hat. Bei jedem Vokal ist zuerst das bis ins archaische Ostseefinnisch zurückreichende Wortgut genannt, das nach seinen finnischen Entsprechungen alphabetisch geordnet ist. Die zweite Gruppe bilden die Wörter, die Entsprechungen im Lappischen haben, aber nicht im Ostseefinnischen, und die dritte diejenigen, die Entsprechungen im Mordwinischen haben, aber weder im Lappischen noch im Ostseefinnischen. Zuletzt kommen die Wörter, die im Tscheremissischen und in den permischen Sprachen vorkommen, aber nicht in den westlicheren finnischugrischen Sprachen. Da unsere Untersuchung sich nicht auf die ugrische Sprachgruppe erstreckt, ist es als in diesem Fall 
überflüssig angesehen worden, die etymologischen Entsprechungen zu erwähnen, die aus diesen Sprachen für unser Wortmaterial beigebracht worden sind. Ebenso sind natürlich solche tscheremissischen bzw. permischen Wörter unberücksichtigt geblieben, die nur in den ugrischen Sprachen Entsprechungen haben. Da das Wortmaterial durchweg allgemein bekannt ist, sind gewöhnlich keine etymologischen Quellenhinweise aufgenommen worden. Zwecks Raumersparnis sind auch die in den ursprünglichen Quellen gegebenen Definitionen der Wörter zwanglos verkürzt worden; ebenso ist, wenn es die Klarheit nur irgend zuliess, die Bedeutung nur beim ersten Wort des jeweiligen Wortartikels gedruckt. Die wenigen etymologischen Beiträge, die der Verfasser jetzt nen hinzugefügt hat, finden sich unter den Nummern 232 (syrj.wotj.), 268 (tscher.). 294, 348 (tscher. $\ddot{a} \cdot r \delta \partial, e \cdot r \delta e$ ), 349, 427 (lp.).

Der Themenkreis dieser Untersuchung ist schon früher ganz. oder teilweise in der Literatur behandelt worden. Kurze Gesamtdarstellungen der Geschichte der Vokale der ersten Silbe im Tscheremissischen und in den permischen Sprachen haben. von der Vertretung in der uralischen bzw. finnisch-ugrischen Grundsprache ausgehend, GoмBocz ("A magyar $a$-hangok történetéhez", NyK XXXIX 229-274; nur die Vokale der urspr. hinteren Reihe werden dort behandelt), Lentisalo ("Zur Geschichte des Vokalismus der ersten Silbe im Uralischen vom qualitativen Standpunkt aus», FUF XXI 5-55) und STEINITz (in dem Werk "Geschichte des finnisch-ugrischen Vokalismus») veröffentlicht. In den Einzelheiten weisen diese Untersuchungen beträchtliche Unterschiede auf, aber gemeinsam ist ihnen ein hypothetischer. weitverbreiter Vokalwechsel als Erklärungsprinzip. Diese Erklärungsweise, die zu wenig die Sonderentwicklung in den verschiedenen Sprachen berücksichtigt, muss als willkürlich und nicht durch Tatsachen begründet angesehen werden. Diese seine Auffassung hat der Verfasser bei seiner Kritik der Steinitzschen Vokaltheorie in dem Aufsatz "Zur Frage nach der Entwicklung des Vokalismus der ersten Silbe in den finnisch-ugrischen Sprachen, insbesondere im Mordwinischen» (FUF XXIX 222-337) begründet. 
Die darin vorgebrachten Stellungnahmen, auf die hier nur hingewiesen sei, ohne sie zu wiederholen, bilden auch den grundsätzlichen Ausgangspunkt der vorliegenden Lntersuchung. Die Analyse des urtscheremissischen Vokalsystems, die Steinitz vorgenommen hat, ist in mancher Beziehung verdienstvoll; z.B. ist hier der Gegensatz zwischen Vollvokalen und reduzierten Vokalen zum ersten Mal klar aufgezeigt worden. Der Teil seines Werkes, der die permischen Sprachen behandelt, ist mehr skizzenartig.

Von den Untersuchungen, die sich ausschliesslich mit dem 'Tscheremissischen befassen, verdient zuerst die schematische Darstellung Erwähnung, in der GExET"z die Entwicklung der von ihm auf Grund der finnisch-lappisch-mordwinischen Beziehungen rekonstruierten Urvokale im Tscheremissischen behandelt ("Unkarin ensi tavumn vokaalien suhteet suomalaislappalais-mordvalaisiin» $=$ Das Verhältnis der Vokale der ersten Silbe im Ungarischen zu denen im Finnisch-LappischMordwinischen, S. 3). Die Tabelle von Genetz, zu der keinerlei Kommentar gegeben wird, ist mangelhaft und sogar fehlerhaft, was grösstenteils durch die geringe Menge und schlechte Qualität des dem Forscher zur Verfügung stehenden Materials verursacht stin dürfte. Die "Cseremisz nyelvtan" von BEкE enthält eine genaue Darstellung der Vokalverhältnisse in den tscheremissischen Dialekten auf Grund des Materials, das zur Zeit des Erscheinens dieses Werkes bekannt war. Die Übersicht ist im wesentlichen deskriptiv. Später hat Beke in seinem Aufsatz "Zur Lautgeschichte der tschuwassischen Lehnwörter im Tscheremissischen", FUF XXIII SS. $66^{-\cdots}$ 84, einige Einzclheiten der tscheremissischen Vokalgeschichte behandelt, u.a. die Frage des Ursprungs des Lautverhältnisses $\mathrm{W} a \sim \mathrm{O} o$. Diese und einige andere Fragen des tscheremissischen Vokalismus waren schon früher von RäsäNen in seiner Dissertation "Die tschuwassischen Lehnwörter im Tscheremissischen" beachtet worden. Das ebenerwähnte Lautverhältnis W $a \sim \mathrm{O} o$ betrachtet auch WIcınsaxx in seiner Kritik von Räsänens Werk (FUF XVI Anz. 32--50) im Licht eines umfangreichen Materials. Wichmanns "Tscheremissische Texte mit Wörterverzeichnis und grammatikalischem Abriss» enthält SS. 124-126 
eine gute Übersicht »Grundzüge der interdialektischen Vokalverhältnisse in der ersten Silbe. (Regelmässige Vertretung.)". die die Grundlage für alle spätere Forschung gebildet hat. Wichmann hat die Vokalverhältnisse in 16 Gruppen eingeteilt. welche Gruppierung noch jetzt wert ist, eingehalten zu werden.

Wenn wir zu den permischen Sprachen übergehen, müsse॥ wir wieder mit Genetz beginnen. Seine Schrift »Ost-permische Sprachstudien» (1897) ist zwar deskriptiv, enthält aber eine phonetisch sehr zuverlässige Darstellung der ostpermischen Vokalvertretung, die unleugbar einen wichtigen Platz in des Erforschung der permischen Vokalgeschichte einnimmt. Die sonderartige ostpermjakische Mundart konnte Genetz als erster Forscher im Jahr 1889 in dem Dorf Paršakova im Uralgebirge kennenlernen; dieses Dorf lag im Wolost Verh-Jazva. das zum damaligen Kreis Cerdyn gehörte, 100 Werst üstlich der Stadt Cerdyn. Die Einwohner dieses Dorfes befanden sich schon zu Genetz' Zeiten in einem Stadium der Russifizierung. Fast gleichzeitig mit der Untersuchung von Genetz erschien Wichuıxss Dissertation "Zur Geschichte des Vokalismus der ersten Silbe im Wotjakischen mit Rücksicht auf das Syrjänische». Dies Werk, dessen Hauptrerdienst in dem reichhaltigen Material liegt, bemüht sich um eine Erklärung der Vokalverhältnisse der ersten Silbe in den permischen Sprachen, indem es von der Grundlage des Urpermischen ausgeht. Leider hat Wichmann mechanisch die im vorhergehenden Jahr von SET̈̈LÄ vorgebrachte Theorie von dem Ablantwechsel der finnisch-ugrischen Vokale angewandt, der mit dem Stufenwechsel zusammenhängen sollte; diese Theorie führte die finnisch-ugrischt Vokialgeschichte für Jahrzehnte auf zum grossen Teil falsche Gleise. Buвrich erklärt in seinem kurzen, aber gerade als grundsätzliche Stellungnahme beachtenswerten Aufsatz " $\mathrm{k}$ вопросу о пермском вокализме» (Бюлтетень JOL KФJ' H 4 . 1929) mit Recht die Erklärungsweise Wichmanns für unbefriedigend. Nach Bubrichs Meinung hat der Vokalismus der ersten Silbe im Wotjakischen noch vor ziemlich kurzer Zeit dem Vokalismus der ersten Silbe im Syrjänischen nähergestanden als es heute der Fall ist; die Ursachen der Auseinanderentwicklung seien einige Lautveränderungen im Wotjakischen gewesen. 
Im Jahr 1930 veröffentlichte der syrjänische Forscher LyтkIx einen Aufsatz "Диалект Кобры" (Соборник комиссии по собпранию словаря и изучению диалектов коми языка, выпуск I, SS. 29 -47), der die Mundart der Ober-Sysola darstellt. Darin macht er darauf aufmerksam, dass es in diesem Dialekt zwei $o$-Vokale gibt, einen "gewöhnlichen" und einen geschlossenen, und zeigt, dass der Unterschied zwischen diesen beiden Lauten, allerdings zum Teil in veränderter Form, im Ostpermjakischen sichtbar ist. Fokos-Fuchs hat in seinem Aufsatz "Adalék a kétféle magyar $e$-hang történetéhez" (Nỵr. LXVI 42-46) seine Aufmerksamkeit auf die Bedeutung der zweierlei o-Vokale im SO-Dialekt für die allgemeine fi.-ugr. Vokalgeschichte gerichtet, vgl. unten S. 279. Von seinen Vorlesungen über die Geschichte der permischen Vokalismus veröffentlichte der Verfasser der vorliegenden Untersuchung im Jahr 1951 in der Zeitschrift "Virittäjä», SS. 440-452, eine Zusammenfassung unter dem Titel "Permiläisen vokaali- ja painotusopin alalta» (Aus der permischen Vokal- und Akzentuationslehre). Sie stimmt inhaltlich überein mit der jetzt erscheinenden Darstellung über dasselbe Thema, wenn sie auch erheblich knapper gefasst ist. Die Akzentverhältnisse sind allerdings darin ausführlicher behandelt als in dieser späteren Arbeit. Schliesslich sind zwei Arbeiten Lytkins, nämlich "Древнепермский язык" und "K вопросу о вокализме пермских языков". Труды Ннститута языюознания I, SS. 58-106, zu erwähnen (beide erschienen im Jahre 1952). In dem die Vokallehre behandelnden Teil der ersteren Untersuchung wird gezeigt. dass einige archaische Züge der Mundart der Ober-Sysola und des permjakischen Dialekts in altsỵjänischen Sprachdenkmälern Entsprechungen haben. Bei der Behandlung der Vokale wird auch den Akzentverhältnissen die gebührende Beachtung zuteil. Im letztgenannten Aufsatz werden die Spezialitäten des Vokalismus der Mundart der Ober-Sysola und des Ostpermjakischen gegen den Hintergrund der urpermischen Verhältnisse ausführlich behandelt. Obgleich dem Verf. diese ausgezeichnete Darstellung erst dann bekannt wurde, als seine eigene Untersuchung schon in Korrektur lag. konnte er sie doch einigermassen in Betracht ziehen. 
FORSCHUNGSMATER IAL.

1. ? TscherO oktâš 'Schlinge, Dohne', syrj. oltini, SO oltini 'eine Falle od. ein Fangeisen aufstellen' $\sim$ fi. ahtaa, lpSchw. ruoktinje, mordM aftan. (Das syrj. Wort ist sicher eine Entsprechung der fi.-lp.-mord. Wortfamilie, aber tscher. oktżš ist schwierig zu beurteilen, weil mundartlich auch eine Form optâš vorkommt, die eine Ableitung rom Verb optem 'stellen, aufladen, laden' sein kann. Wotj. oltini: juäz o. 'in die Tenne führen (das Getreide)' dürfte vom syrj. Wort getrennt sein, vgl. Verf., Kalevalaseuran Vuosikirja 31 S. 105.)

2. Syrj. (Wied.) vojny 'durchgehen od. ausreissen mit etw.,

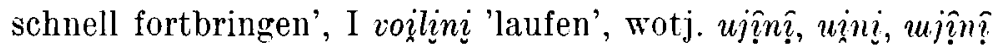
$\sim$ fi. ajaa, lp. vuoggjet.

3. 'Tscher. $\ddot{u} l-, \ddot{u} l \dot{\delta}$ 'das Untere, Unter-', syrj. $u l, u v$, wotj. ul, uи $\sim$ fi. ala, lp. vuolle, mord. al.

4. Wotj. ibini, îb? n⿳⺈ 'schiessen' $\sim$ fi. ampua, lp. ablbot.

5. Syrj. udni 'tränken, zu trinken geben', wotj. udịn? $\sim$ fi. antaa, lp. vuowldet, mord. andoms.

6. 'TscherW $\grave{o} \cdot \beta \hat{\partial}, O$ (Vasiljev) oßjo 'Schwiegervater' $\sim$ fi. appi, lp. vuop'pâ.

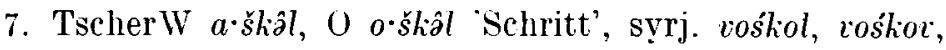
PO úśkol, wotj. (Wied.) utśkyl $\sim$ fi. askel, mordM aśkâllis ( E eśkellis).

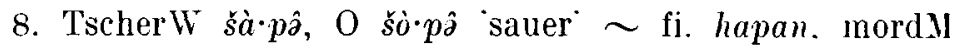
šà̀amă, E tšà̀amo.

9. Syrj. jukni 'teilen, trennen', wotj. Tukini, jukni $\sim \mathrm{fi}$. jakaa, lp. juokket, mord. jacoms.

10. Syrj. juskini 'abspannen', wotj. juskịn? jutsk?̣̂? $~$ fi. jaksaa, lpK ja $\bar{a}_{i} k s e-(<\mathrm{karel}$. ?). - N. B. mordM juksândâ'sich entschuhen, die Fussbekleidung abziehen', das $u$ in der ersten Silbe hat und am liebsten für eine Ableitung von einem urspr. Stamme ${ }^{*}$ joksa- zu halten ist.

11. TscherW jal, O jol 'Fuss' $\sim$ fi. jalka, lp. juollge, mord. jalgo.

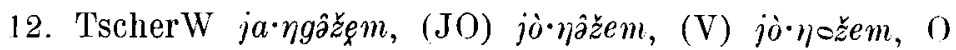


jònôžét’m 'kauen, wiederkauen, mahlen' $\sim$ fi. jauhaa, mord. jažams.

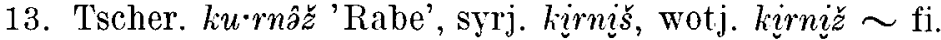
kaarne, lp. gārânâs, mordE kírentš, M krandâs.

14. Tscher. $k o \cdot \delta e m, \operatorname{ko} \delta e^{\cdot} m$ 'lassen, zurücklassen, verlassen', syrj. kolni, sO kolni, PO kül-, wotj. kiqlini, kįlni, (MU) litini $\sim$ fi. kadota, lp. guoddet, mord. kadoms.

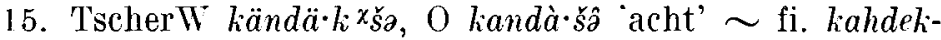
san, lp. gavce, gakilce-, mord. kavkso.

16. TscherW ko.em, O kue.m 'graben, schaufeln', syrj. koini, SO koini, wotj. kujani fi. kaivaa, lp goailvot. - Im Lappischen, anders als im Ostseefinnischen, ${ }^{*} o$ in der ersten silbe.

17. 'Tscher. kok, ko·ktât 'zwei', syrj. kịk 'zwei', I kikina-miss, I' kikjà'-mis 'acht' (aber in anderen Dialekten kclijà-mis), wotj. kik, kilkt- fi. kaksi, lp. guokilte, mord. karto.

18. 'I'scher. kol 'Fisch' $\sim$ fi. kala, lp. guolle, mord. kal.

19. Syrj. kulem, I kulem '10-15 Klafter langes Netz mit Flössen und Netzsenkern, usw.' $\sim$ fi. kalin.

20. Syrj. goz, SO goz'Paar', wotj. kuz fi. liansa, lp. ga $\bar{j}^{\lfloor} z^{e}$.

21. TscherW $k a \cdot n d e m$, O konde $m$ 'bringen' $\sim$ fi. kantaa, !p. guodldet, mord. landoms.

22. Wotj. kuațş̌àni, kuašstàni zusammenstürzen, zerfallen' $\sim$ ? fi. katketa.

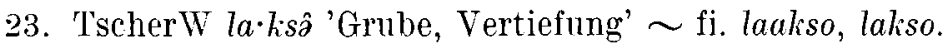

24. Syrj. lud, rud 'Viehweide, Wiese', wotj. lud fi. lansi, est. laas.

25. 'TscherW (K) mâšte $m,(J)$ mo•štèm, O moštę $m$ 'können, sich auf etw. verstehen' $\sim$ fi. mahtaa, mord. maštoms. 26. Syrj. moi 'Biber', wotj. mîn $\sim$ fi. majava, lp. maggjeg, mord. mijav ( $i \mathrm{im}$ Mordwinischen unregelmässig, wenigstens teilweise unter dem Einfluss des folgenden $j$ entstanden).

27. Tscher. mokx xs 'Leber', sỵj.-wotj. mus $\sim$ fi. maksa, IpSchw. muokse, mord. makso.

28. Tscher. mör, mò̀ rỏ 'Beere, Gartenerdbeere' $\sim$ fi. marja, lp. muorlje, mord. maŕ.

29. TscherW na.tšliâ, O no.tśliâ 'nass; regnerisch; Regenwetter' $\sim$ fi. nahkea, lp. njuoskâs, mord. natško. 
30. Tscher. $n \grave{u} \cdot \delta \hat{a}$ 'jüngere Schwester des Mannes od. del' Frau' $\sim$ fi. nato, $\operatorname{lpS} n \hat{\sigma} t{ }^{\prime} \varepsilon<$ fi. ?

31. TscherW pa•rmâ, O po•rmâ 'Pferdefliege, Bremse' fi. paarma, parma.

32. Syrj. bad', baidd 'Weide' 〜 fi. paju, ? $\operatorname{lpI}$ póăjò̆̀ (im Lappischen $*_{o}$ ).

33. Sỵrj. penni 'Beischlaf ausüben', wotj. pon?̣n? 'legen. anlegen, stellen, setzen' $\sim$ fi. panna, ? mord. pańems 'backen'.

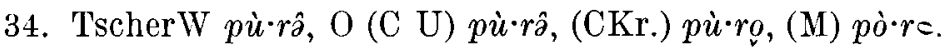
(B) poro', (BČ) pò ro 'gut, gesund; das Gute'; W parè $\cdot m a ̈ m$. $\mathrm{O}$ (C U) parema $\cdot m$, ( $\mathrm{M}$ B) porema'm 'genesen', syrj.-wotj. bur $\sim$ fi. para-: paras, lp. buorre, mord. paro.

35. Syrj.-wotj. pur 'Floss, Fähre' entweder fi. pari 'Schwarm, Schar' oder lp. boarlre (<* porava) 'Floss'.

36. TscherW pat, O pot 'Topf, Kessel' $\sim$ fi. pata, lp. batt $<$ fi. ?

37. TscherW (K) šo am, (JO V) šu am, O šua $m$ 'kommen'.

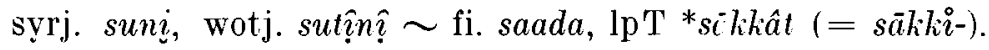
mordE sajems, sams, II ścvams, sajams.

38. Syrj. śorni, PO śórni 'Rede, Gespräch' fi. saarna 'Predigt', est. sarnane 'ähnlich', lp. sardne < fi.

39. ? Syrj. suk 'dicht, dick' $\sim$ fi. sakea, lp. suokkâd.

40. TscherW šò $l \hat{\partial}$ 'Dieb', W šòlâštam, O šolšta $m$ 'stehlen $\sim$ fi. sala, lp. suolle, mord. salams.

41. Tscher. šol, šò $\cdot l \hat{\partial}$ 'Ulme' $\sim$ fi. salaca, mordE śeleri, śelej. II säti (im Mordwinischen sekundäre Palatalisierung).

42. Syrj. śor, SO šor 'Sparren', wotj. śurị $\sim$ fi. sarja, est sarikas.

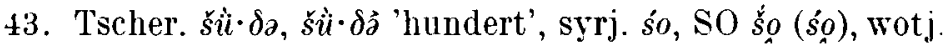

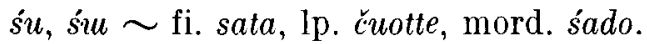

44. Wotj. tịlis, tịl?̣s 'Wiesen-, Feld- od. Waldhütte' $\sim$ fi. tala, talas.

45. TscherW $t u m, O t \grave{u} \cdot m \hat{a}$ 'Eiche' $\sim$ fi. tammi, mord. tumo.

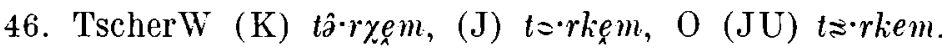
(CMU) turke $m$ 'ausstehen, aushalten, dulden, ertragen (z.B Kälte)' $\sim$ fi. tarjeta, tarkene-

47. Syrj. turin, turun 'Gras, Kraut: Heu', wotj. turịm. turị̂n $\sim$ fi. tarna, taarna, est. tarn. 
48. Syrj.-wotj. dugdini 'aufhören' $\sim$ fi. tauota, taukoa-. lp. duowlgot.

49. TscherWO ßaštar, O (JT M) ßoštar 'Ahorn' fi. vaahtera, vahtera, mord. ukštor.

50. Syrj. veini 'sinken', veitni, vetni 'senken', PO vijj-'untersinken', vót- 'eintauchen, ins Wasser senken', wotj. vîi? ni $\sim$ fi. vajota, lp. vuoggjot.

51. TscherW (K V) $\beta a \cdot l e m,(\mathrm{JO}) \beta o \cdot l e m$, O Bole $\cdot m$ 'helabsteigen, herunterklettern, herabsinken' $\sim$ fi. valkaa, valkama, lp. vuollget, mord. valgoms.

52. TscherW $\beta a \cdot l \gamma \partial \hat{\partial} \hat{a}, \mathrm{O} \beta 0^{\prime} l \gamma \partial \hat{\partial} \hat{\partial}$ 'Helle, Klarheit; hell. klar' $\sim$ fi. valkea, lp. viel'gâd (sekundäre Palatalisierung). : mord. valdo.

53. Syrj. važ 'alt', wotj. vuž $\sim$ fi. vanha.

54. Syrj. $v u g:$ ibe $\cdot s-v$. 'Türgriff', wotj. $v u g \sim$ fi. ranko. vanku, vanka, est. vang.

55. TscherW (K) $\beta u \cdot r \delta \hat{z},(\mathrm{JO} \mathrm{V}) \beta=\cdot r \delta o,(\mathrm{JP}) \beta \approx \cdot r \delta=$. 11 $\beta u \cdot r \delta \hat{\partial},(\mathrm{CKr}.) \beta \approx \cdot r \delta o,(\breve{C}) \beta a ̂ r t o ~ ' S c h a f t$, Stiel' $\sim$ fi. varsi.

56. Syrj. vartni 'dreschen, schlagen', vartan 'Dreschflegel' $\sim$ ?? fi. vartta, varsta 'Dreschflegel'.

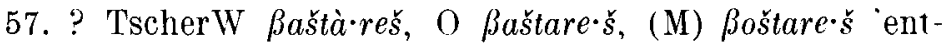
gegen; gegen, wider' $\sim$ fi. vasta- 'gegen-', lp. vuos'te-. : mordM vastă 'Stelle, Platz, Ort; Schlafstätte, Bett'.

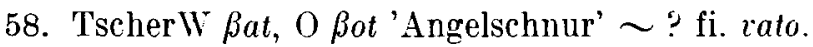

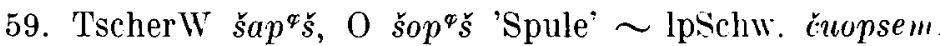

60. TscherW ka•tškam, O kotška $\sim$ lp. gas lket.

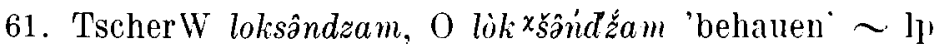
luok'čât, mord, lakśems.

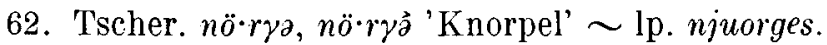

63. TscherW na.šmâ, O no.šmâ 'Gaumen, Kiemen', ș̣rjl noktšim, S nektśim, L loktśim lp. njuovčâ -klčâm-.

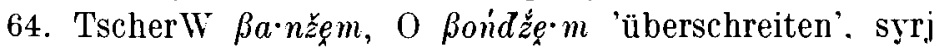

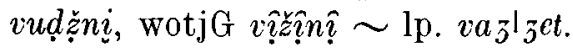

65. Tschero ßotkem 'entrinden (Birke od. Weide)' $\sim \mathrm{lp}$.

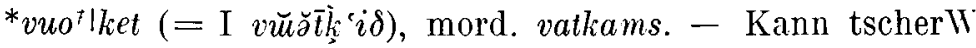
Baktam, O Boktam 'entrinden' wirklich eine metathetische Form ron ßotkem sein? Vgl. Beke NyK XLV 340. 
66. TscherW $a^{-}: a m, \grave{a} \cdot \gamma \hat{\partial} m$ usw., $\mathrm{O} o^{-}: o^{\prime} m, \dot{o} \cdot \gamma \hat{\partial} m$ usw., verneinendes Verb $\sim \operatorname{mordE} a$, at, M af 'nicht'.

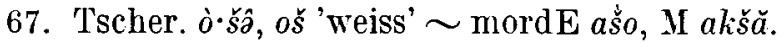

68. Syrj. ozir, SO ozir, L uzir 'reich', wotj. uzir $\sim$ mord. $\operatorname{azor}(o)<$ ar.

69. Syrj. vož, SO òz 'Deichsel', wotj. uajị̂z, vajẹ̌z $\sim$ mord. ažija, ǎ̌ja, ažj $\bar{\varepsilon}$. - Das Wort stammt vielleicht vom Arischen her:? *aiša. S. ToIvoxen FUF XIX 99.

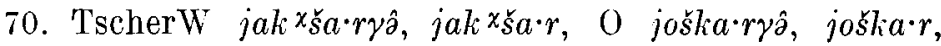
(U auch) jöškarye 'rot' mord. jakstere.

71. Tscher. kì̀·zâk 'Mitgift', syrj. koźin, SO koźin, PO kýzin, wotj. kużim $\sim$ mord. kaźems; kaźne, kaźme.

72. TscherO moštem 'müde werden', ? syrj. mudźni, I P midźni $\sim$ mord. maštoms 'verloren gehen; umkommen, verschwinden, ein Ende nehmen'.

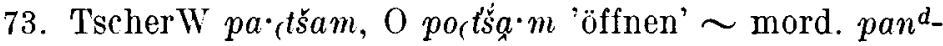
žoms.

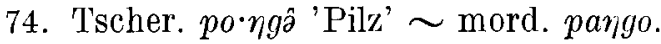

75. Syrj. sudźni 'reichen' itr., wotj. surdżini $\sim$ mord. saźi .

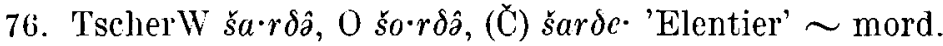
śardo.

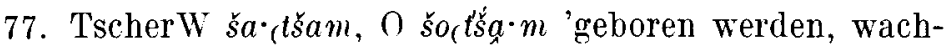

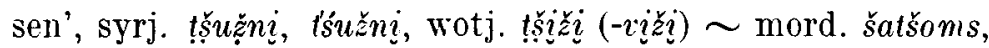
tšatšoms.

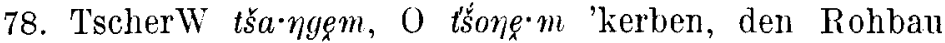
zimmern' $\sim$ mord. tšavoms, šavâms 'schlagen, erschlagen, prügeln'. - Dass das fi. Wort haava 'Wunde' hierher gehört, ist nicht wahrscheinlich.

79. Tscher. to šstâ 'alt (nicht neu)' $\sim$ mord. tašto.

80. TscherW $\beta a z$, O $\beta$ o $z$ 'Wurzel', syrj. vuz, wotj. vižzi, ข?ใน?

81. TscherW $j a \cdot \delta a m, ~ O ~ j o \delta a \cdot m$ 'fragen', syrj. juvalni, jualni, wotj. juàni.

82. TscherW $p a \cdot n d \hat{a}, \mathrm{O}$ po•ndâ 'Strauch, Pflanzenstengel', wotj. pud.

83. TscherW patš, O potśs 'Schwanz', sỵrj. bę̌, PO byž, wotj. biž, bîz. 
84. TscherW raž, O roź 'Loch', syrj. roź, SO ròź (VL U I ruź).

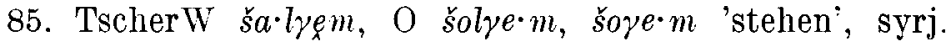
sulalni, sultni, wotj. sultîn ?, sultini; silini, silni.

86. 'TscherW ša'ptàr, O šo'ptâr 'Johannisbeere', syrj. seter, sęter, PO sétor, wotj. suter, sutär.

87. TscherW juž, 0 jù.žâ 'jemand' $\sim$ fi. joka, joku, lp. juokke, mord. ju- : juza toza.

88. Syrj. ju 'Fluss', wotj. ju: j.-sur $\sim$ fi. joki, lp. jokkâ, mord. jov. Lp. $\check{o}<{ }^{*} u$.

89. Syrj. juk' 'Haufen', wotj. juk, luk $\sim$ fi. joukko, lp. joawlko $<$ fi.

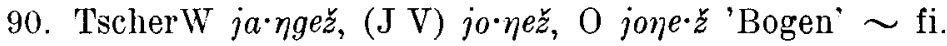

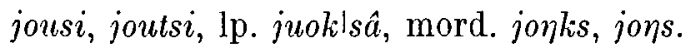

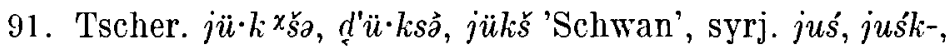
PO jyś, wotj. juś, juś $\sim$ fi. joutsen, lp. njuklčâ, mord. loksiij, lokśtim, lokśti.

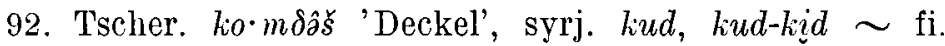
kansi (der Vokalismus unregelmässig), lp N goawlde, T kiâmte, mord. kundo.

93. Syrj. kia, kiva 'Röte am Himmel' $\sim$ fi. koi.

94. SyrjI kịr 'männlicher Hund' $\sim$ fi. koira.

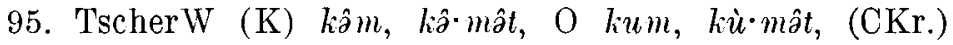
$k: m, k s \cdot m \hat{\imath} t$ 'drei', syrj. kujim, PO kvim, ord. koinmed, SO koimed, P kvimét 'drei'; ko-min, SO komin 'dreissig', wotj. kuinn(m-), kuinmè $\cdot \hat{\imath} ;$ kù̀-min $\sim$ fi. kolme, lp. golb'mâ, ord. goalmad, mord. kolmo. Vgl. S. 202.

96. TscherO kordem 'beräuchern; beim Opfern den Tisch mit einem brennenden Kienspan umkreisen' $\sim$ fi. kortata, kortaa, lp. goarldet, mordE kur'tams, kirtams, II kâr'ta'ms.

97. Syrj. kor, SO kor, PO kur 'Blatt', wotj. kuar $\sim$ fi. korva 'Ohr', korvalehti 'Ohrzipfel, Ohrmuschel'.

98. TscherW $k a \cdot s ̌ k \hat{\partial}, \mathrm{O}$ (U) $k a \cdot s ̌ k \hat{a}$, (UJ) koške, (USj US) kośkâ 'Stromschnelle', syrj. koś, kośk fi. koshi, lp. guoilkâ.

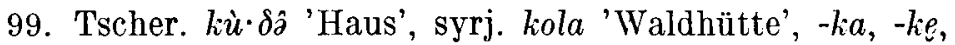
-ko, -ku : kerka, kerku 'Haus', vitśke, vitśko, vitśku 'Kirche', wotj. kua, kuala fi. kota, lp. goatte, mord. kud(o). 
100. 'Tscher. kutškâž, kutkâž 'Adler', syrj.-wotj. kuț̣̌ $\sim$ fi. liotka, lp. goas|kem, mord. kut'skan.

101. Tscher. kot 'Jahr, Zeit', ?? syrj. kad fi. kotva, IpSchw. kåtfo.

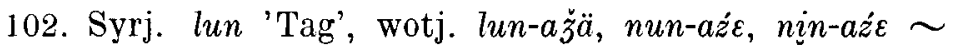
ii. louna, lounas.

103. Syrj. (P) munda 'wieviel?; soviel wie'; -min: ki-min

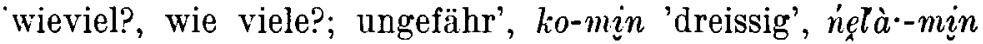
'vierzig'; -minda : si-minda 'soviel', wotj. minda 'soviel wie', linà-min 'dreissig' $\sim$ fi. moni.

104. Tscher. n⿳亠े冋 $\cdot r a, n \dot{o} \cdot r \grave{\partial}$ 'biegsam, geschmeidig' $\sim$ fi. norja.

105. Syrj. nuni, nulni 'tragen, forttragen', wotj. nuini, nuni $\sim$ ? fi. noutaa.

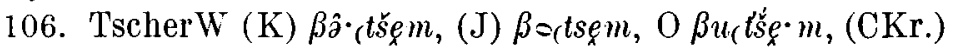
$\beta s_{\text {it }}$ 'sem 'warten' $\sim$ fi. odottaa, lpSchw. ådsotet, mord. utšoms.

107. 'Tscher. ßui 'Kopf' $\sim$ fi. oiva, lp. oai ve.

108. Tscher. $u k \chi_{s}$ 'Ast, Zweig',? wotj. usi 'Egge' $\sim$ fi. oksa, Ip. oak Ise.

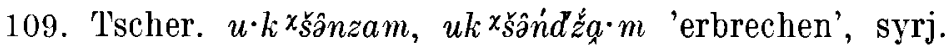
rosni, U vesni, SO osni, $\mathrm{P}$ esni, $\mathrm{PO}$ ysst-, wotj. eskini, MU ossini $\sim$ fi. oksentaa, lp. vuoklset, mord. uksnoms.

110. 'Tscher. $\grave{u} \cdot l \hat{\partial}$ 'es gibt, es ist'; W $\hat{\partial} \cdot l a m, \mathrm{O} u l a \cdot m,(\mathrm{CKr}$.)

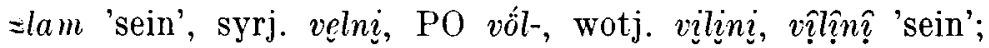
ral, vaiu 'war' $\sim$ fi. olla, mord. ulems.

111. 'Tscher. $u r$ 'Eichhörnchen', syrj. $u r \sim$ fi. orava, $1 p$. uarlre, mord. $u r(o)$.

112. 'TscherO uža 'Preis'; W (K) $\beta^{2} z ̌ a \cdot l e m,(J)=z ̌ a \cdot l e m$, 1) užalę· $m,(\mathrm{CKr}$.) ₹žalem 'verkaufen', syrj. vuz:vuz-vot 'Steuer, Abgabe', vuzalni 'verkaufen', vuzęs 'zu verkaufende Ware', wotj. vuz, vuzàni, vuzäs $\sim$ fi. ostaa, lp. oas'tet, oases.

113. TscherW $a \cdot n z \partial \hat{l}, \mathrm{O} \quad o^{\circ}$ 'ndżàl 'das vorn gelegene, das vordere, vorder-', syrj. vodź, SO vòddź, P odź, PO uź, wotj. aź, adź $\sim$ fi. otsa, liv. vēntsa.

114. ? Syrj. (auch SO) votni, PO vót- 'pflücken, sammeln; wickeln, knäueln' fi. ottaa, est. võtma.

115. Syrj. pož, SO pòz, PO puž 'Sieb'; požnalni, SO pòznalni, PO púznal-, wotj. puz, puž fi. pohtaa, pohtia, mord. ponžavioms. 
116. Tscher. püe•ryo 'Knabe, Mannsperson', syrj.-wotj. pi $\sim$ fi. poika, mord. ṕijo, bujo.

117. TscherW (K) $p u \cdot l-\beta u i, p a \hat{l} l-\beta u i,(\mathrm{~J} \mathrm{~V}) p u l-\beta u i$, O $p u l-$ $\beta u \cdot i,(\check{C} \mathrm{JT}) p \in l-\beta u i$ 'Knie' $\sim$ fi. polvi, lp. buollvâ, mordM pâlma $n^{d} \tilde{z} \varepsilon, \mathrm{E}$ pul'aza.

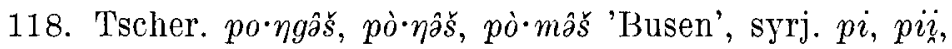
wotj. pij, pi, (Wred.) pöi $\sim$ fi. povi, lp. buog $\hat{a}$, mord. pongo, pov, povă.

119. Tscher. šur 'Horn', syrj. śur, wotj. śur, śur $\sim \mathrm{fi}$. sarvi, lp. coarlve, mord. śuro. - Lp., mord. <*sorva; die Vokalvertretung des fi. Wortes unregelmässig.

120. Tscher. šolkamá, šâlkamà 'Schnalle an der Hemdbrust', $\because$ syrj. śul: dod'-s'. 'Schlittenkufe', ? wotj. śul $\sim$ fi. solki, lp. ćulgum, mord. śulgamo.

121. TscherW tsa'ma, O t'soma. 'junges Hengstfüllen', syrj. t'śán, t'san $(m)-$, wotj. t'suni $\sim$ ? fi. sonni.

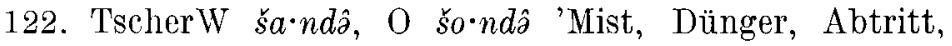
Urin' $\sim$ fi. sonta.

123. TscherO (MK) šu $s e \cdot m$ 'verfluchen, verwünschen'; W

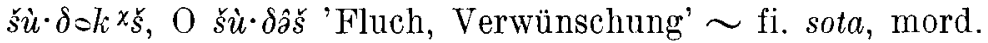
śudoms.

124. TscherW šò̀ : ala--šoè 'eine bunte Ente', O šue 'récze', sue 'Gänsejäger', syrj. śul-t'śež, wotj. śulî $\sim$ fi. sotka, lp. čoailge, mord. śulgo.

125. Tscher. šol, šò là 'Darm', syrj. śul, śuv, wotj. śul, śu $\sim$ fi. suoli, lp. čoalle, mord. śulo. - Lp., mord. < ${ }^{*}$ śola; die Vokalvertretung des fi. Wortes unregelmässig.

126. TscherW (J) to ktâ-lo: $\delta \circ$ 'Seetaucher (Colymbus arcticus)', syrjU tokti $\sim$ fi. tohtaja, lp. dovtâ - $k \mid t a \hat{a g}$-.

127. Tscher. tošta'm 'wagen' $\sim$ fi. tohtia, lp. duos ttât.

128. ? TscherW torlém 'zurechtweisen, schelten' $\sim$ fi. tora, torua, lp. doarro, doarrot, mord. turems.

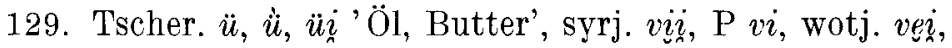
(MU J) vö $\sim$ fi. voi, lp. vuoggja, mordE oj, M vaj.

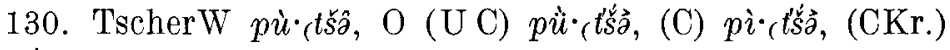
$p \hat{\imath}_{t} t^{\prime \prime} \varepsilon,(\mathrm{M})$ pù $\left(t_{s} \circ\right.$ 'Renntier', (syrj. pez 'junges, ungehörntes Renntier' wahrscheinlich < ostj.) wotj. pužei $\sim$ lp. boa $z^{\circ}$.

131. Syrj. pert, PO pyrt 'Kessel', wotj. purti $\sim$ lp. boarlte 
$\left(<{ }^{*}\right.$ portta) 'Gefäss aus Birkenrinde'. (Fi. pursi 'Boot' ist aus lautlichen Gründen hiervon getrennt zu halten.)

132. T'scher. to $r e \check{s}$, tore $\cdot \check{s}$ 'quer' $\sim$ lp. doares, mordE turks.

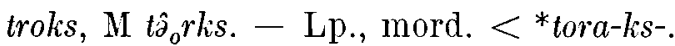

133. Tscher. ko škęm, koškę $m$ 'trocknen, trocken werden'. syrj. kośmini, sO kośmini, wotj. kuaś, kuaśmini $\sim$ lp. goikes. mord. kośke.

134. Syrj. ṅèz 'träge, langsam' $\sim$ lp. njoacce $<{ }^{*}$ no 135. SyrjPO nimál 'Hase' $\sim$ lp. njoammel, mord. numolo. - Lp., mord. <* nomala.

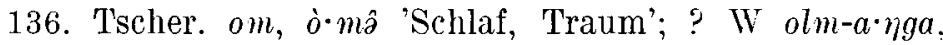

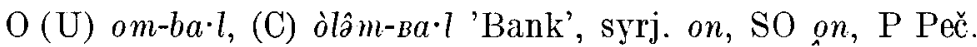
I U PO un 'Schlaf', wotj. un, um, (U) ? $m \sim$ lp. oadzet, mord. udoms. - Lp., mord. <*oda-.

137. Tscher. šok $x_{s}$ 'Ärmel', syrj. soi, SO soi 'Arm'; sos. SO sos, PO sus 'Ärmel', wotj. sui 'Arm', sajes 'Ärmel' $\sim$ lp. soaggje, soaggja $\left(<{ }^{*}\right.$ soja $)$.

138. 'Tscher. šuk $\chi_{s}$ 'Wurm' $\sim 1 p$. suoklsâ, mord. suks. Urform wahrscheinlich *sokse. - Im Mordwinischen mag suks statt ${ }^{*}$ soks darum entstanden sein, damit das lautliche Zusammenfallen des Wortes mit soks 'Ski' vermieden wurde.

139. Tscher. muš 'gebrechter Hanf' $\sim$ mord. muško < * moška.

140. Syrj. nur 'Mehlzusatz zur Suppe' mord. norox. noru.

141. Syrj. nort, (I) nort, (L) nork, PO nort 'langer Schlitten. usw.', wotj. nurt $\sim$ mord. nurdo $<{ }^{*}$ norta.

142. Syrj.-wotj. $p i: p i$-pu 'Espe' $\sim$ mord. poj, poju.

143. Tscher. $p u$. štâ 'Brei' $\sim$ mord. pušto < * pošta.

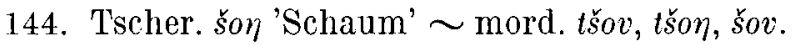

145. Tscher. šož 'Gerste', syrj. ț̣̌uz, t'suž, wotj. tššžjem $\sim$ mord. tšuž, šuž. - Urform wahrscheinlich *tšsoša bzw. *š้oša.

146. Syrj. tul, tuv 'Nagel, Pflock, Zapfen, Keil', wotj. tui $\sim$ mord. tulo $<*$ tola.

147. 'TscherW o'škô 'Pappel', O oško 'Schwarzpappel; Esche' $\sim$ mord. ukso, uks < ${ }^{*}$ oska $\left({ }^{*} o k s a\right)$. Vielleicht indogermanischen Ursprungs. 
148. TscherW $\beta a z$, O $\beta$ ǒ 'Verzweigungspunkt, Verzweigung', korno-ßoz 'Scheideweg', syrj. voz 'Flussarm am Oberlauf, grosse Gabel, gabelförmige Teilung, gabelförmig geteiltes Ende (eines Gegenstandes), Ast, Nebenfluss', tuin-voz 'Wegscheide, Wegkreuzung', SO kok-voz'Zwischenraum der Beine'

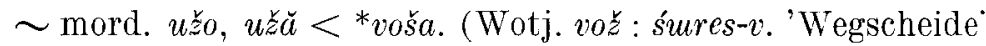
darf man nicht -- wie es z.B. UotiLA Syrj. Wortschatz s.v. vož tut - hiermit verbinden, weil seine lautgesetzliche Entsprechung syrj. vež : tui-v. 'Wegkreuzung, Wegscheide' ist.)

149. Tscher. $k u \cdot k$ žsa 'trocken', syrj. kos, SO kos, P kes, PO kys, wotj. kes.

150. Tscher. kù ·žâ 'lang', syrj.-wotj. kuź.

151. Tscher. no'lyâ'Ulme', wotj. nulo.

152. Tscher. o'ptem, opten $m$ 'bellen', syrj. utni, uvtni, wotj. utini, utni, uutini. - Liv. utà'b, das allgemein in diesen Zusammenlang gestellt wird, ist seiner Vokalvertretung $(u)$ nach etwas befremdend und kann anderen Ursprungs sein. Beide Wortfamilien sind natürlich ursprünglich deskriptiv.

153. Tscher. $\grave{o} \cdot z \hat{z}$ 'Hengst', syrj.-wotj. už.

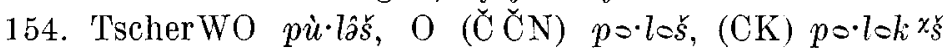
'Schulterbein, Schultergelenk, Achselwurzel; Oberarm; Schulter', syrj. puly : p.-pom.

155. 'Tscher. ko.lem, kolęm 'sterben', syrj. kulni, kuvni, wotj. kulini, kulni $\sim$ fi. kuolla, mord. kuloms.

156. Tscher. koz 'Fichte', syrj. koz, SO koz, P kez, PO kyz, wotj. kị̂z fi. kuusi, lp. guossâ, mord. kuz. - In diesem Worte gibt es zwei Typen: *kūse (> fi., mord.) $\sim{ }^{*} k \bar{o} s e$ (>lp.).

157. Tscher. nòे là : n.-pik $\times$ š 'Pfeil (mit knöcherner Spitze)',

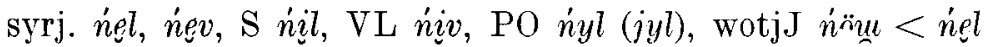
$\sim$ fi. nuoli, lp. njuollâ, mord. nal.

158. Syrj. pul, puv 'Preiselbeere' $\sim$ fi. puola, puolukka.

159. Syrj. rot's, SO rot's, PO rut's' 'Russe', wotj. d'źut'ś < ostseefi. *rōtsi bzw. *ruotsi.

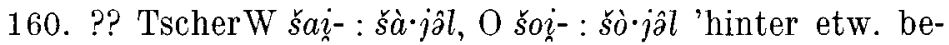
findlich', syrj.-wotj. sai $\sim$ fi. suoja, lp. suoggje < fi. ?

161. Syrj. sol, sov, SO sol, PO sul 'Salz', wotj. silal $\sim$ fi. suola, mord. sal.

2 - Finnisch-ugrische Forschungen 
162. TscherW (K) süm, (J V) šnnm, O šüm, (CK Č) šöm 'Fischschuppe', syrj. śem, PO śym, wotj. sem $\sim$ fi. suomu, lp. čuobmâ, mord. śav.

163. TscherW šün, O šün, sün, śün, šön, sön, (CMU) šin 'Sehne', syrj. segn, wotj. sẹn, (U) sịn $\sim$ fi. suoni, lp. suodnâ, mord. san.

164. Tscher. $l o \cdot m b \hat{a}$ 'Faulkirsche, Traubenkirsche', syrj. lem, POl'ym, wotj.l'em fi. tuomi, lp. duobmâ, mordM lajme, Elom.

165. SyrjI volōni, Ud. vola vnis, P vovavni, PO vólal-, S velalni, L vela $\cdot 7 n i, \mathrm{~V}$ velavni '(mit dem Messer) hobeln,

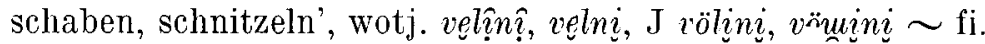
vuolla, Ip. vuollât, fuollât.

166. Syrj. ver, PO vyr 'Wald', wotj. vir 'Hügel, Anhöhe' $\sim$ fi. vuori 'Berg'.

167. Syrj. vo, SO vo 'Jahr'; vośa, SO ośa, PO úśa 'Sommergetreide', meim-u, PO möjm-u 'im vorigen Jahre', wotj.

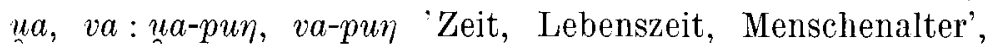
(MU) $u$ in tauböris' 'folgendes Jahr' $\sim$ fi. vuosi.

168. Tscher. tù·mâš 'Flicklappen', syrj. dęmni, degmas,

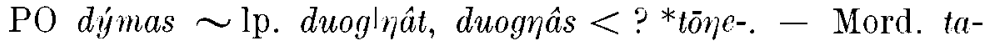
vadoms 'bedecken' ist aus lautlichen Gründen davon getrennt zu halten.

169. Syrj. seskini 'kauen', wotj. siskini, sịskîn ? $\sim$ lp. suosikât, mord. suskoms. - Die Urform wahrscheinlich *sōske-.

170. Tscher. šùer, šua·r 'hölzerner Mörser' fi. huhmar, huumar, mord. tšovar, šovar.

171. Syrj. ši, ši 'Spiess, Stachel', wotj. ši, (MU) $̌ ّ i \sim f i$. hui, est. hoi, oì, liv. vgi, lpSchw. ${ }^{*}$ suoggjâ (= $\mathrm{P}$ su.ojja $)$. - Im Worte gibt es zwei lautliche Varianten: ${ }^{*} u \sim *_{o}$ (> est., liv., lp.).

172. Syrj. (auch SO PO) jon, jonm- 'stark, kräftig', wotj. jun $\sim$ ?? fi. julma 'grausam'.

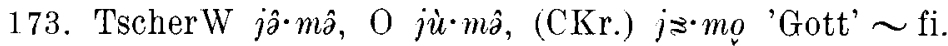
jumala.

174. TscherW kâ-, O $k u-$, (CKr.) $k \gg-$ Pronominalstamm,

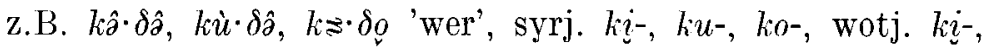
$k \hat{\imath}-, k u \sim$ fi. $k u-$, lp. gu-, ko-, mord. ko-. - Die permischen 
Stammvarianten können zum Teil Fortsetzungen des urspr. Stammes *ko- (z.B. fi. ko-s-ka, lp. goa-s 'wann') sein.

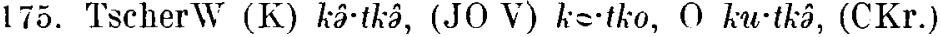
$k: t k o$ 'Ameise', syrj. kot : koddźul-k., k.-ko,dźul, SO kot : ko(dżul-k. est. kuklane, liv. kukki, lp. gotlkâ, mord. kotkodov.

176. Syrj. kilalni 'stromabwärts treiben' $\sim$ fi. kulkea, lp. gollgât, mord. kolgams, kol'gems.

177. Syrj. kim: sin-k. 'Augenhügel, Augenbraue', wotj. kimes, kimis fi. kulma, lp. gullbme < fi.

178. TscherW $k \hat{a} \cdot m \hat{a} k$, U $k \grave{u} \cdot m \hat{a} k$, (CKr.) $k \approx m s \cdot k$ 'verkehrt, umgestürzt', syrj. kimmi, wotj. kimàni $\sim$ fi. kumo : kumossa, lp. gobmo : gobmot, mord. komams.

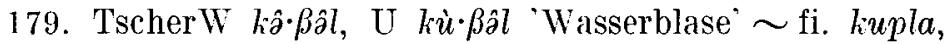
lp. *gowlle (= lpI ko B̀le).

180. Syrj. kusni 'auslöschen, erlöschen', wotj. kị̂s?̂n? $\sim$ est. *kus- : kustuma, lpR kopse-.

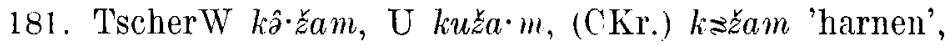
syrj. kud'ź, PO kyj, wotj. kid'ź, kị̂z, (U MU J) kiż fi. kusi, lp. gu $\check{z}|\check{z} \hat{a}, g \circ \check{z}| \check{z} \hat{a}$.

182. Syrj. kul'mini 'laichen' $\sim$ fi. kutea, lp. gođdât.

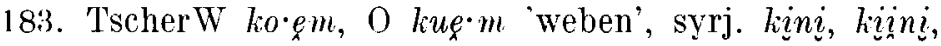
wotj. kuini $\sim$ fi. kutoa, lp. goddet, mord. kodams.

184. 'TscherW ka.ja, O koja. 'Fett', wotj. lei $\sim$ fi. kuu $\left.1<{ }^{*} k u i-\right)$, mord. kuja, kuj $\varepsilon^{\circ}$.

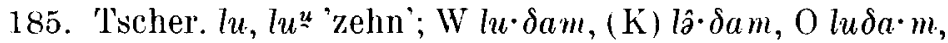
(CKr.) $l=\delta a m$ 'rechnen, lesen', syrj. lid, wotj. lid lịd $\sim$ fi. lukea, lp. lokkât, mordE lovoms, XI lucâms.

186. 'TscherW lâm, O lum, (CKr.) lミm 'Schnee', syrj. lịm, rim $m$, wotj. li $m \underline{i}, \operatorname{li} m \hat{\imath} \sim$ fi. lumi, lpS $* \operatorname{lob} m \hat{a}(=$ Wefs. $л u p m \varepsilon)$ $(<$ fi. ?), mord. lov, lor .

187. TscherW (K) lâेmęm, (JO V) lomem, (K) lo.mem, O lume $m$, (CK С̆) $l=m e m,(\mathrm{JT}) l 0^{\cdot} \cdot \mathrm{mem}$ in der Ausdrucksweise s!nzä'm là'mem'durch Hexerei verblenden' $\sim$ fi. lumo, lumota.

188. T'scher. $l u, l u^{u}$ 'Knochen', syrj. $l i$, wotj. $l \underline{\sim} \sim$ fi. $l u u$,

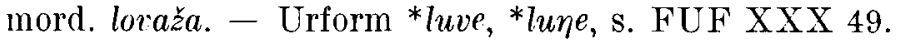

189. TscherW (K) môsškam, (JOV) ms.škam, O muška m,

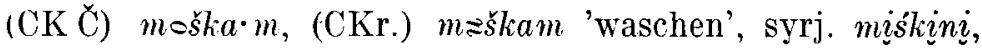
wotj. miśkị̂̂? $\sim$ est. mõskma, mord. muśkems. - Est. $\tilde{o}<*^{*}$. 
190. TscherW mo.am, O mua.m 'finden', ? syrj. moid, (auch PO) mod' 'Märchen', (auch SO) moidni 'Sagen erzählen', ? wotj. mad', mad?̣̂ni $\sim$ lüd.-olon. muja-, fi. muistaa, est. mõista, liv. moìstâ (im Ostseefinnischen also Doppelheit $u \sim o$ ), mord. mujems.

191. TscherW $m \hat{\partial} \cdot n \hat{\partial}$, O $m \dot{u} \cdot n \hat{\partial},(\mathrm{CKr}$.) $m \boldsymbol{\imath} \cdot n \emptyset$ 'Ei' $\sim$ fi. muna, lp. monne, mânne, mord. mona.

192. Syrj. něs, PO nyš 'Schlegel, Klöpfel', wotj. nuši. nuctș̣̂̂ $\sim$ ?? fi. nuija, (dial.) nuhja.

193. TscherW nôेlem, O nule $m$, (CKr.) nalem 'lecken'. syrj. nulni, wotj. ńulîn $\hat{\imath} \sim$ fi. nuolla, lp. njoallot, mord. nolams. - In diesem Worte sind die Vokalverhältnisse wahrscheinlich auf Grund von dessen affektivischer Bedeutung - ganz unregelmässig: $*_{\bar{o}}$ (ostseefi.) $\sim *_{o}$ (lp.) $\sim *^{*}$ (mord.).

194. Syrj. biț̣̌kini 'stechen', wotj. bitş̌kaltini $\sim$ fi. puhli, puhjeta, puhkaista.

195. TscherW pân, O pun, (CKr.) $p \approx n$ 'Haar, Daunen. Feder' $\sim$ fi. puna, mord. pona.

196. TscherW pâ·nęm, O pune्x·m, (CKr.) pænem 'zwirnen; flechten', syrj. pinnni, wotjG punịn $\hat{\imath}, \mathrm{ML}^{\top}$ pinini $\sim \mathrm{fi}$. punoa, lp. bodnet, bâdnet, mord. ponams.

197. Syrj. pirid'z' 'Brecheisen. Eishaue', wotj. pyrič $\sim$ fi. puras.

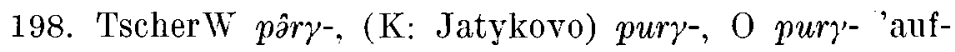
wirbeln, einstöbern', syrj. pira, pirżini, wotj. purdżizin $\sim \mathrm{fi}$. purku, lp. borlgâ.

199. TscherW pâेram, O pura $m$, (CKr.) psram 'beissen. kauen', syrj. purni, wotj. purini $\sim$ fi. purra, lp. borrât, mord. porems.

200. Syrj. pìdźirtni 'ausdrücken, auspressen', wotj. piźżtini $\sim$ fi. pusertaa.

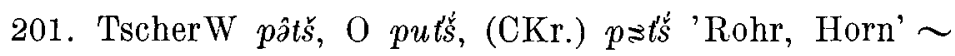
fi. putki, lp. boslkâ, mord. potško.

202. TscherW (K) š̀̂lla, (JO V) šolà, (JP) šs.là, O sulà, šulà, (CK Č) šolà. 3. Sg. Prs. 'tauen, schmelzen', syrj. sîl, siv; siln $\underline{i} \sim$ fi. sula, sulaa, mord. solams. 
203. TscherW šâ·rem, O šurèn, (CKr.) šærem 'stossen, stampfen' $\sim$ fi. survoa.

204. Tscher. šu: W $i \cdot m-s ̌ s, ~ O ~ i \cdot m \grave{\partial}-s ̌ u$ 'Nadelöhr', ? wotj. śu : ś. kwaśmem 'das Dursten' $\sim$ fi. suu, lpS *čuvvâ $(=\check{c} u w-$ $w \varepsilon)$. - Wahrscheinliche Urform *suve, obgleich auch *s'u möglich ist. Vgl. FUF XXX 50.

205. TscherW tâl, O tul, (CKr.) tミl 'Feuer', syrj. tìl, tiv : t.-kert 'Feuerstahl, Feuerzug', wotj. tịl, tịl $\sim$ fi. tuli, lp. dollâ, mord. tol.

206. 'Tscher. to.lam, tola'm 'kommen' $\sim$ fi. tulla, $1 \mathrm{pK}$ *dollât $(=$ tolli-) $<$ fi. ?

207. Tscher. ton, to'mo, tom 'Kern' $\sim$ fi. tuma, mord. tov, ton.

208. Syrj. tedni, PO tö́d- 'wissen', wotj. todịn $\hat{\imath} \sim$ fi. tuntea, lp. dowldât.

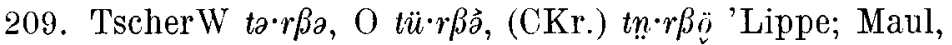
Schnauze', syrj. tirp, wotj. tirpi $\sim$ fi. turpa, mordE turva, torva, M târva.

210. TscherW $\beta a \cdot \delta a r$, O $\beta o \delta a \cdot r$, (CK C) $\beta a \delta a \cdot r$ 'Euter' $\sim$ fi. udar, utare, mord. odar.

211. Tscher. $i \cdot \ddot{a} m, \ddot{i j a} \cdot m$ 'schwimmen', syrj. uini 'waten, schwimmen', wotj. uj?̣̂n $\sim$ fi. uida, est. uju-, (dial.) oju-, "jo-, lp. vuoggjât, mord. ujems. - Im Worte hat es eine ${ }^{*} u$ und eine $*_{0}$-Variante gegeben. Die erstgenannte wird wenigstens von fi. uida, est. uju-, die letztere wenigstens von est. oju-, lp. vuoggjât vertreten.

212. SyrjI $i<* i l$ 'Zeltstange' $\sim$ fi. ulku, lp. (h)ollgâ.

213. Syrj. $o b$ 'heftiges Schneegestöber', ? wotj. $o \cdot b \varepsilon$ 'vollends' $\sim$ ?? fi. umpi, ?’ lp. oblbâ.

214. Syrj. vunni 'vergessen werden', wotj. vunini $\sim$ ? fi. unohtaa 'vergessen'. - Eine andere Möglichkeit ist, dass fi. unohtaa eine Ableitung von uni 'Schlaf, Traum' ist.

215. 'Tscher. ì, uu 'neu', syrj. vill, vil, P vil, wotj. vịl, vil $~$ fi. uusi, lp. ođâs, oddâ, mord. od. - Das Lappische und das Mordwinische deuten, anders als das Ostseefinnische, auf urspr. kurzes $* u$ hin.

216. Syrj. til, tiv 'Schwung- oder Schwanzfeder', wotj. tili $\sim$ Ip. dollge, mord. tolga. 
217. Syrj. kįzn! 'husten', wotj. kị̂zin? $\sim 1 p$. gossât, mord. kozams.

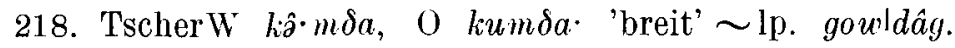

219. 'TscherW kâेmęm, O kumę $m$, (CKr.) $k s m e m$ 'die Augen schliessen, syrj. kunni, wotj. kị̂nị̂? $\sim$ mord. konams. - Fi. kyyny passt als vordervokalisches Wort sehr schlecht in diesen Zusammenhang.

220. TscherW $l \hat{a} p^{\varphi} \breve{s}, \quad$ O $l u p^{\varphi} \breve{s},(\mathrm{CKr}) \quad. l \approx p s ̌ s ~ ' P e i t s c h e ' \sim$ mord. lokšso.

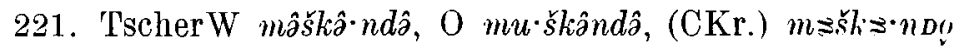
'Faust', wotj. mižzig mord. mokšânda, mokšna.

222. TscherW mô·rem, O mure•m, (CKr.) marem 'singen' $\sim$ mord. morams.

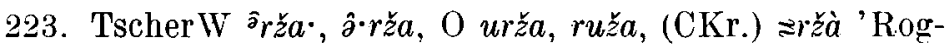
gen', syrj. ru,dźeg, rucdźeg, wotj. dźiżeg, dźeg mord. roź.

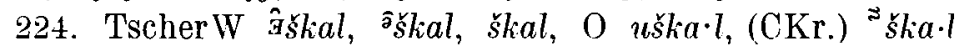
'Kuh', wotj. iskal, sikal mord. skal.

225. TscherW mâz, O muž 'Krankheit', syrj. mižz, wotj. $m \underline{i} z$.

226. TscherW (JO V) no ly $\diamond,(\mathrm{JP}) n \approx \cdot l \gamma \diamond, 0 n u \cdot l \gamma \hat{\partial},\left(\mathrm{J}^{\prime} \mathrm{T}\right)$ $n \diamond^{\prime} l \gamma o$, (P B M UJ CU்) nolyo ' 'Edel-, Weisstanne' (o in del' osttscher. Variante nolyo rührt offenbar von einer Vermischung mit no.lyó 'Ulme' her), syrj. nịl, n.-pu, wotj. nill-pu.

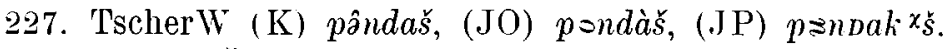
O pundàs, (CK ל̆) pondàš 'Grund. Boden', syrj. pidess, wotj. pides.

228. TscherW pầrem, O purę'm, (CKr.) parem 'hineingehen, eintreten', syrj. pirni, wotj. pirini.

229. TscherW pâš, O puš, (CKr.) psš 'Kahn, Boot', sỵrj. $p i z$, wotj. $p \hat{\imath} \tilde{z}$.

230. TscherW $t \hat{\partial} \cdot k t \hat{\partial}$ 'Spant, Rippe', syrj. tikk.

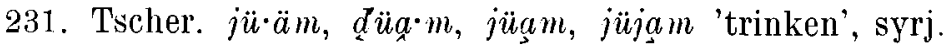

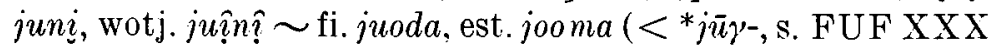
5-6), lp. jukkât.

232. Syrj. jur 'Kopf, Haupt, wotj. jir $\sim$ ? fi. juuri, : mord. jur.

233. Tscher. ko.lam, kola·m 'hören', sỵj. kilni, kivni, wotj. kilini, kị̂ln̂? $\sim$ fi. kuulla, lp. gullât, mord. kulems. 
234. Tscher. kut, kù ·dât 'sechs', syrj. kvaint, kvat', wotj. k'uat', kwat' fi. kuusi, lp. guttâ, mord. koto. - Im Worte Doppelheit $* \bar{u}(>$ fi., Ip. $) \sim * u(>$ mord.).

235̃. Tscher. $p u$ 'Brennholz, Holz', syrj.-wotj. $p u \sim$ fi. puu. Vgl. FUF XXX 1--2.

236. Tscher. šue $m$ 'rudern', syrj. sinni $\sim$ fi. soutaa $(<$ *sū $\gamma$-ta-, s. FUF XXX 13), lp. sulkât, suwldet.

237. Tscher. tul 'Sturm', syrj. tel, tev', PO tyl, wotj. tel,

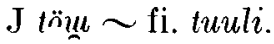

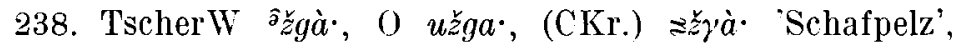
syrj. $i z$, PO $\theta z$, wotj. $i z, \hat{z} z \sim$ fi. $u u h i$.

239. 'Tscher. kon 'Aschlauge', syrj. kun, $k u n(m-) \sim 1 p$. gudnâ.

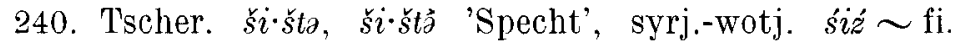
hähnä, häähnä, lp. čaihne.

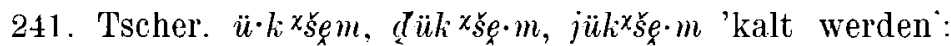

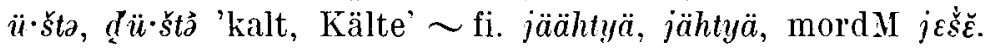

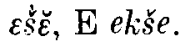

242. TscherW (K) jär, O jer 'See' fi. järvi, lp. jau're, mordM $j \varepsilon \dot{r} k_{\varepsilon} \breve{\varepsilon}, \varepsilon \dot{r} k \breve{\varepsilon}$, E eŕlie.

243. TscherW (K) jè Knötchen einer Pflanze', syrj. jez 'Volk, Leute, Menschen', jez-vi, Le jez-vi 'Sehne, Gelenk', PO jöz' Zeit', jöz-aj 'Schwie-

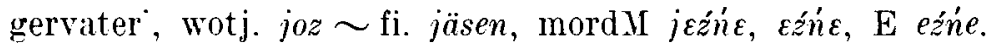

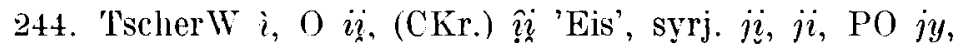
wotj. je $\sim$ fi. jäa $(<* j a ̈ \eta e)$, lp. jiegrâ. mordM $j \varepsilon j, \varepsilon j, \mathrm{E} e j$, $e v, e \dot{\eta}, i j$.

245. T'scher. ke·läm, liela·m 'waten', syrj. kelni, PO köl-, wotj. kolîn $\hat{\imath}$, kolni $\sim$ fi. kaalata, kahlata, lp. gallet, mordM kel'ams, E k'elems. - Urform nach der lappischen und mordwinischen Vertretung *kälä-. Im Ostseefinnischen exzeptionell $a h, \bar{a}$ statt $\ddot{a}$.

246. Syrj. kel 'Schwägerin', wotj. kali $\sim$ fi. käly, lp. gālŏjedne, mordM kel.

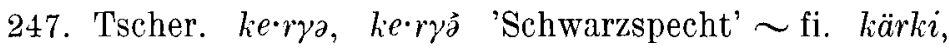
palokärki, mordM käríää.

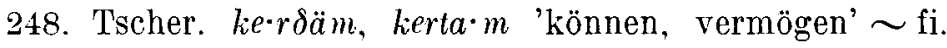
kärsiä, lp. gierldât, mord. hiŕdems. 
249. TscherWO kit, O (C) kât 'Hand', syrj. ki 'Hand'; ke-piś, PO ki-pis 'Fausthandschuh', wotj. li $\sim$ fi. käsi, lp. giettâ, mordM ked, E ked.

250. Syrj. køsjịni, PO kốsji- 'versprechen, wollen', wotj. kosini $\sim$ fi. käskeä.

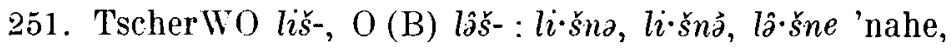
in der Nähe', usw. fi. lähi- : lähellä, läsnä.

252. TscherW (K) lä·ktäm, (J) le ktäm, O lekta $m$ 'hinausgehen', sỵj. lokni, lokt-, SO lokni, PO lók(t)-, L lakni, lakt-, wotj. liktini $\sim$ fi. lähteä, $\operatorname{lpK} *$ liekltât $(=$ likte- $)<\mathrm{fi}$.?

253. ? Syrj. let's, PO leć 'Schlinge, Dohne' fi. lämsä, lp. law! ̌̆e.

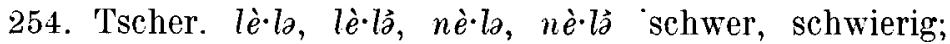
Schwere' $\sim$ fi. läyli, lps *liewlâs < fi. ?

255. 'Syrj. malalni 'anfühlen, betasten' $\sim$ estn. mälestada, wot. mäleht $\vec{a}$.

256. Syrj. remis' 'Wiedergekäutes', remiśtini 'wiederkäuen', wotj. džomeśtini $\sim$ ? fi. märe, märehtiä.

257. Wotj. naani (< na-al-) 'nachsehen, schauen, betrach-

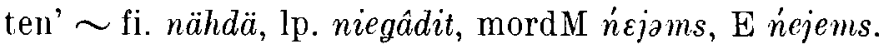

258. T'scherW nìna, O nì•nà, (B) nâne 'diese' (pl.), syrj. $n a \sim$ fi. nämä, mordM ń $\varepsilon$, nat, $\mathrm{E}$ ne, net.

259. 'Tscher. pük $\times s$ 'Haselnuss', wotj. paš : p.-pu, p.-muli; puš : p.-moli $\sim$ fi. pähkinä, mordM ṕcšť̌, E ṕešte.

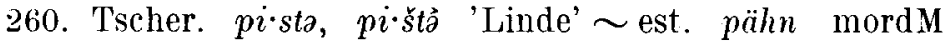
päšä, E pekšse.

261. 'Syrj. bi 'Feuer'; bia, bija 'feurig', biva 'Zunderbüchse' fi. päivä, lp. bailve.

262. Syrj. pek, PO pök 'Rogen' ? fi. päkikä.

263. Syrj. (auch SO PO) pon, pom 'Ende, Anfang, Spitze',

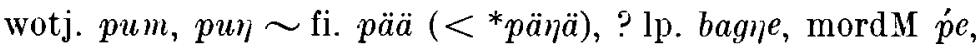
E ṕpe, p̈ä.

264. Syrj. reped 'Rauchloch', wotj. džopi fi. räppänä, reppänä, est. räpp, räpen, lp. rappen.

265. Syrj. sep, PO söp 'Galle', wotj. sep, (WIED.) söp fi.

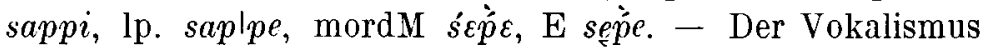
les Wortes im Ostseefinnischen ist ganz eigenartig; alle anderen Sprachen weisen klar auf die Urform *säppä hin. 


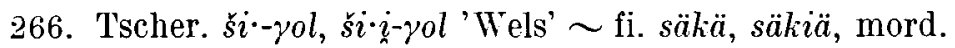
sïje, śijä.

267. TscherW ̌̌ä $\eta g \partial$ 'dürrer Ast eines Strauches od. eines kleinen Baumes'; $p u-s ̌$. 'wachsender Baum', $O \check{s} e \cdot \eta g \grave{\partial}$ in $p u-s ̌$. ibid. $\sim$ fi. sänki.

268. TscherW š̈̈ryem, O šerye.m 'öffnen, zerstreuen', ? wotj. (WIED.) sery-, serty-, sörty- 〜? fi. särkeä, ? mordM sääräda-, E sęrede-.

269. Tscher. šerena, šere ngà 'Rotauge, Plötze' $\sim$ fi. särki, mordM säárgäa, E ş̨ŕge.

270. Syrj. sin 'Kühling, Rohrkarpfen', wotj. son-t'šrig $\sim$ fi. säynäs, säyne, säynäjä, $\mathrm{lpK} * \operatorname{sie} n n \hat{a}-d n a \hat{g}-(=\operatorname{sivn} \mathrm{G}$.

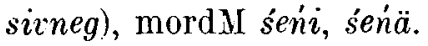

271. Tscher. tel, tè̀là 'Winter', syrj. tel, PO töl, P (WiED.) tol, wotj. tol $\sim$ fi. talvi (das fi. Wort exzeptionell hintervokalisch), lp. dallve, mordM tală, E t'el'e.

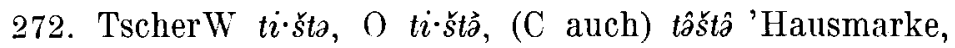
Namenszeichen' $\sim$ fi. tähti, lpR ${ }^{*}$ das'te $\left(=\mathrm{T} t \bar{a}_{i} s t e\right)$, mord M tešt

273. Tscher. ti, tii 'Laus', syrj. to $i$, SO toi, PO tyj, wotj. tei, (Munk.) täj $\sim$ fi. täi.

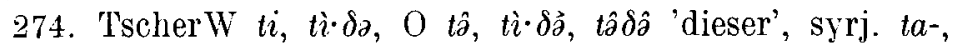
taje, wotj. ta fi. tämä, lp. dät 'dieser' (bzw. diet 'dieser hier'), mord $\mathrm{M} t^{\prime} \varepsilon, \mathrm{E}$ ie.

275. Syrj. ti 'Lunge', wotj. ti $\sim$ fi. täry (täty), mord. tevillav. 276. 'TscherW tsits, O titš, (CKr.) tẹtśs $\sim$ tîts's 'voll' $\sim$ fi. เäysi, $\operatorname{lpK} \mathrm{K}^{*}$ diew'dâ (= tivt, tivt, [attr.] tivdas, tivtas)<? ostseefi. 277. Tscher. $\beta i, \beta i i$ 'Kraft', syrj. -vi:jez-vi, wotj. $-v i$ :

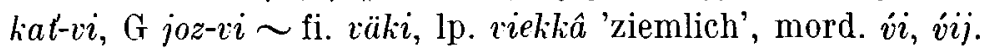

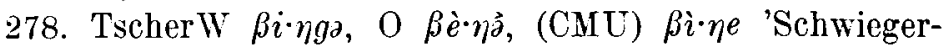
sohn', ? syrj. von, PO vun 'Bruder', ? wotj. vin, vị̂n 'jüngerer Bruder' fi. vävy, lp. vivvâ (< ? fi.), mordM ov.

279. Syrj. ai 'Vater, Grossvater', wotj. ai, aji $\sim$ fi. äijä, lp. aglgja.

280. Tscher. $i m, i \cdot m \grave{a}$ 'Nadel', syrj. jem, PO $i m \sim \mathrm{fi}$. äimä, lp. ailbme.

281. Syrj. oktini, $\mathrm{PO}$ ókti- 'abhauen, fällen' $\sim 1 \mathrm{pO}{ }^{*}$ akltet (= Sk. $\dot{a} \bar{z} \bar{t} e D)$ 'schlachten'. 
282. Tscher. $p \grave{e} \cdot l \partial, p \grave{e} \cdot l \grave{\partial}$ 'halb; Hälfte, der eine von einem Paar', syrj. pel, PO pöl 'Seite; eines von einem Paar', wotj. pal, pån 'Seite, Gegend; halb, Hälfte' lp. balle 'Seite: halb, Hälfte', mordM ṕl' $\bar{\varepsilon}$, E pele 'halb, Hälfte'.

283. Syrj. śelktalni, PO sólltal- 'Bastschuhe flechten od. flicken', wotj. śektànị (dial. śi-) lp. čiekltât, čikltât, čik'tet (im Lappischen ein interdialektaler Wechsel $i e \sim i$; der erstere. zweifelsohme ursprüngliche Typus erscheint im Pite- und Inarilappischen).

284. Syrj. lésédni, PO löśst- 'ordnen, bereiten, anpassen'. leśalni 'passen, tauglich sein', wotj. leśalni $\sim \operatorname{lp.~laččet~}(<$ : *läśä-).

285. 'Tscher. mel 'Brust', wotj. mil, mîl: m.-aź $\sim \mathrm{lp.}$ miellgh, mordM mäl'liä. (Das Wort melki der nördlichen Dialekte Finnlands ist aus dem Lappischen entlehnt. Woher stammt das fi. Wort mälli, das in PAasoxess Ost-tscher. Wb. s.v. mol erwähnt wird?)

286. 'TscherW (K) mäk x̌̆, (J V) mèk $x_{s}$, O mek $x_{s}$ 'trockenes faules Holz $\sim$ lp. mieslkât, mieskâs.

287. TscherW $j ! \cdot l m a, j a \cdot l m a, O(\mathrm{U}) j \vec{\partial} \cdot l m \vec{a},(\mathrm{BČur} . \mathrm{BC̆})$ jâlme, (P) ṅàlme, (UP) jïlmâ 'Zunge, Sprache $\sim$ lp. njallbme $\left(<{ }^{*}\right.$ ńälmä).

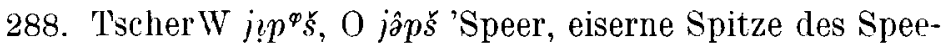

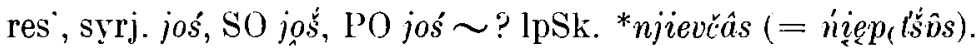
Das Lappische deutet ziemlich klar auf eine Urform mit * $\ddot{a}$ iu der ersten Silbe hin.

289. Syrj. ses 'Schmutz, Unreinigkeit, schmutzix, garstig'. wotj. ses $\sim 1 \mathrm{pO}{ }^{*}$ saklse (= T sā $\left.\bar{a}_{i} k e\right)$, mord. ş̨ks, sękśe. seksă.

290. Tscher W e.ltem, O öltż, elte. 'ein Armvoll', sỵrjF eve.dź, PO óliź, wotj. al $\sim \operatorname{mordM} a ̈ l^{\prime}, j a ̈ l$, E el, eleśt'.

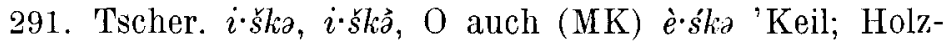
pflock' mordM äskä, E eske.

292. 'Tscher. kem 'Stiefel', syrj. kem, PO köm mordV

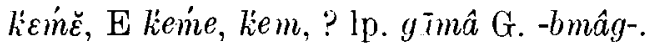

293. Tscher. kiš 'Fichtenharz, Harz' $\sim \operatorname{mordM} k i \varepsilon s ̌ c ̌ s, \mathrm{E}$ kekiše.

294. Syrj. seźnis 'ausziehen, herausziehen, ausreissen, abreissen' $\sim$ ? mord $\mathrm{X}$ śźzms, E seźems 'reissen, abreissen, zer- 
reissen'. (Hinsichtlich des anlautenden Konsonantismus besteht die Inkonsequenz syrj. $s \sim \operatorname{mord}$. $s$.)

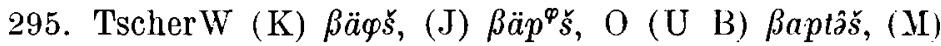
ßopt $\lesssim s ̌$ (o unregelmässig) 'Jagdnetz zum Fangen von Hasen. Hasennetz', syrjU vetes, wotj. (Muxk.) votäs.

296. TscherW (K) $\beta \ddot{a} \cdot r \gamma a,(\mathrm{JO} \mathrm{V}$ ) $\beta \dot{e} \cdot r \gamma \partial$, () $\beta e \cdot r \gamma \grave{\partial}$ 'Niere', syrj. verk.

297. TscherW (K) ki žęm 'frieren; sich erkälten', syri.

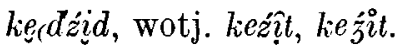

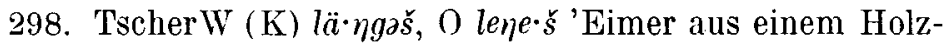
stück', wotj. laฑez.

299. Tscher. l’̀ $p_{\partial}$, lep 'Milz', syrj. (auch s() P(O) lop, wotj. lup.

300. 'L'scherW lü·lpa, O lölpö (lörpö, nölpô, nörpâ) 'Erle', syrj. lol-pu, PO lom-pu, wotj. lul-pu, (MU J) lunu-pu.

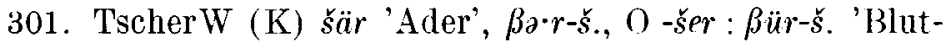
ader', wotj. ser : vir-s.

302. TscherW se'rmats, O še rmàtś, šörmnntśs 'Halfter', syrj. sermed, PO sérmot, wotj. sermet.

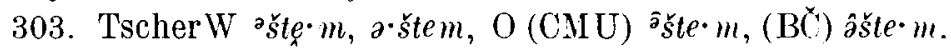
(CKr.) Îstem 'machen, tun', syrj. eštini, P ještinni, PO jéšti$\sim$ fi. ehtiä, lp. âsltât.

304. TscherW a.lem, O (U C) ile $\cdot m$, (UJ) $i 7 e \cdot m$, (M) $i t e m$.

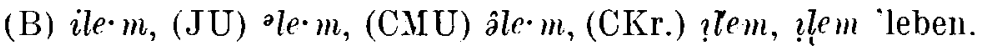

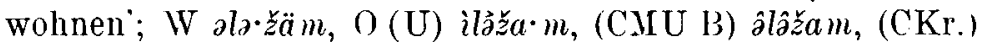

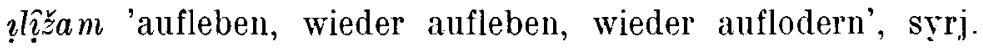
(auch SO) olni, PO ól-, wotj. ulini, ulịn $\imath \sim$ fi. elää, lp. rellet. 305. Tscher. $k \ddot{u} \cdot \ddot{a} m$, liüa $m$ 'sieden, reifen' $\sim$ est. keema $\left(<{ }^{*} k e i-\right.$, s. FUF XXX 45), fi. keittää.

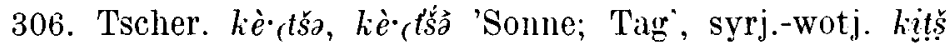
$\sim$ fi. kehä, est. keha, kihä, kiha, mord. ši, tši, tšsi.

307. TscherW kü, O kö, ke 'wer', syrj.-wotj. kin, kin $\sim \mathrm{fi}$. ken, lp. gi G. gēen, mord. ki, kije.

308. TscherW kar, O kür, (CKr.) knn 'Lindenbast', syrj. kor, SO kor, wotj. kur fi. keri, lp. gârrâ, mord. keŕ, käŕ, $k \varepsilon r$.

309. Syrj. (auch SO) korni, PO kór- 'bitten, einladen, herbeirufen', wotj. kurini $\sim$ ? fi. kerjätä. 
310. 'Tscher. ke•r $\delta_{\partial}: p \ddot{u} l \ddot{a}-\gamma e \cdot r \delta \partial$ 'vor ziemlich langer Zeit',

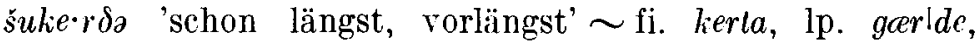
mordM kârda., E kirda $<$ balt.

311. ? Syrj. kos, kosk-, SO kos, PO kusk 'Kreuz (am menschlichen Körper), Lenden, Taille, Mitte des Körpers', wotj. kus $\sim$ fi. keski, mord. kieskă.

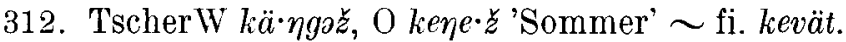

313. TscherW la॰štäš, O (UP) listaš, (USj) lištaš, (MK)

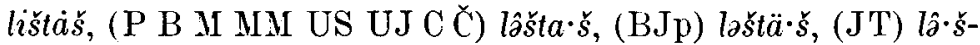
taś 'Blatt' $\sim$ fi. lehti, lp. lâsltâ.

314. Tscher. lü.en, lüe $\cdot m$, lüje $m$ 'schiessen', syrj. liinni,

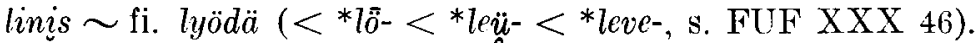

315. Syrj. lol, lov, SO lol, PO lul 'Atem, Geist', wotj. lul, lul $\sim$ fi. löyly $\left(<{ }^{*}\right.$ levls $)$, lp. liewllâ.

316. 'TscherW $m \ddot{a}$, O me 'wir', syrj.-wotj. $m i \sim$ fi. $m e$, lp. mi, mord. min.

317. Tscher. mük $\chi_{s}$ 'Biene', syrj. moš, V PO (mala-)muš, wotj. muš, muš $\sim$ fi. mehiläinen, mord. meš, mekš.

318. 'Syrj. mal' 'Ruder' $\sim$ fi. mela, lp. melle, mord. milä.

319. Tscher. $m i \cdot e m, m i e \cdot m, ~ m i j e \cdot m$ 'kommen, gehen', syrj. munni, PO munz, wotj. munini, minnini, mị̂ṇ̂̂ $\sim$ fi. mennä, (dial.) männä, est. minema, lp. mânnât.

320. ? Tscher. mü, mü $i$ 'Honig', syrj. ma, wotj. mu, mu $\sim$ fi. mesi, lp. miettâ (<? fi.), mord. med. Das tscher. Wort gehört vielleicht in einen anderen Zusammenhang, s. TorvoNeN JSFOU 56: 1 S. 20.

321. Syrj. nil, niv 'Tochter, Mädchen', wotj. nil, nîl $\sim \mathrm{fi}$. neiti, neito, lp. nieildâ.

322. TscherW nal, O (U BČur.) nâl, (CKr.) nı̣l, (M) nil, (UP) $n \grave{\imath} \cdot l a \hat{t}$, (BČ) nil 'vier', syrj. nol, SO nol, PO nul id.;

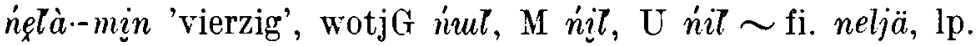
njalllie, mord. nile.

323. Syrj. pesni, PO ṕes- 'schlagen, klopfen, prügeln', wotj. posị̂n $\sim$ est. peksma (< ${ }^{*}$ peks $\left.\ddot{a}-\right)$, fi. pieksää, piestä (fi. ${ }^{*} \bar{e}$ dürfte sekundär gegenüber est. $e$ sein), mord. ṕiksâms, pivięms, pivtsęms.

324. Syrj. polni, poini, SO polni, PO púl- 'fürchten, sich 
fürchten', wotj. pulyny, puuiñ $\sim$ fi. pelätä, lp. bâllât, mord. pelems.

325. Tscher. pi, pi, pi⿱亠 'Hund'; W panè $\gamma \hat{a}$, O (JU M) $p i-$

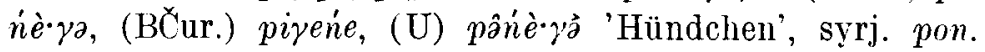
SO pon, PO pun, wotj. puni, puni, (U B) pq̣n? $\sim$ fi. peni. penikka, lp. b̄̄enâ G. -dnâg-, mord. ṕine.

326. Syrj. ber, PO bör 'zurück, wieder; Hinterteil', wotj. ber, (WIED.) bör fi. perä, est. pära, mord. ṕir $\varepsilon$, ṕira, ṕŕ $\varepsilon$.

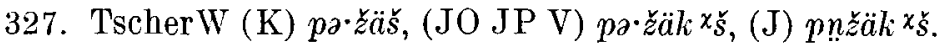

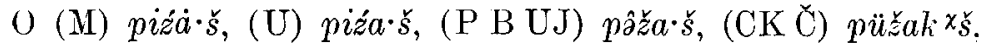

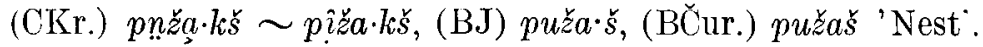
syrj. poz, SO pòz, PO poz, wotj. puz, (MU) piz $\sim$ fi. pesä, lp. basse, mord. ṕiză, ṕizę.

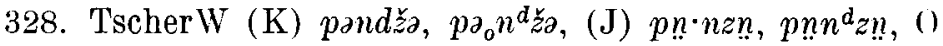

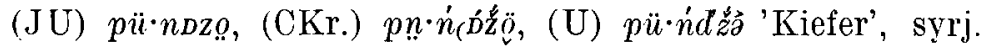
(auch SO) požem, wotj. pužim $\sim$ fi. petäjä, lp. bacce, mord. pitše, pitšs.

329. TscherW (K) raßaž, $\partial r \beta \partial \check{z},(\mathrm{~J}$ V) $r n \beta n \underline{z}, \mathrm{O}(\mathrm{P}$ B M UJ

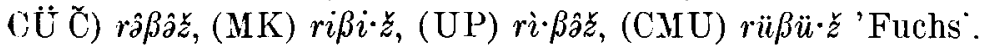

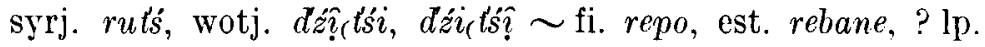
rievân, mord. riveś, irvíśs.

330. 'TscherW s’̀ $\delta \partial$ 'der, jener' $\sim$ fi. se, mordE śe.

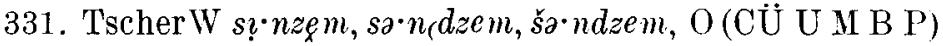

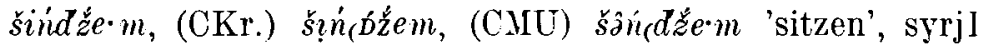
sidźni $\sim$ fi. seisoa, seistä. - Daneben gibt es hintervokalische Formen: estS saizma, 1p. éuo z̆l žot.

332. TscherO (M W Iснм.) šiši m, (M BEKE) šâšsââse, (P GEN.) šâšâm 'sieben', syrj. śiżim, sizziim, wotj. sizżim $\sim \mathrm{fi}$.

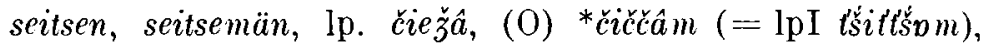
mord. siș'em.

333. TscherW (J) šalaž, O (JU) šalaž, (U) šŝ.lâz, (BČr. BČ) šâlâz 'der untere Teil des Rückens, das Kreuz', (M) ši la 'Rücken' $\sim$ fi. selliä, lp. čiellge.

334. Tscher. šü, šüi 'Hals', syrj. śi (-keď̌); śili, silili, wotj.

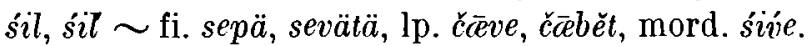

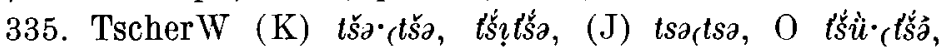
(CKr.) t's̆n $t^{\prime} s_{\hat{u}} \cdot$ 'Oheim, Mutterbruder', syrj. (auch SO) t'soz, ț̣̆oz̆, wotj. ț̦̌uz $\sim$ fi. setä, lp. čacce, mord. tšitše. 
336. Syrj. śoini, SO šoini, PO śúj- 'essen, fressen'; sodni,

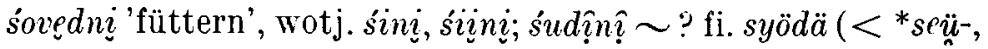
* seve-, s. FLF XXX 46), ? mord Éśems, sävims, M śevams, śivams.

337. 'T'scherW tä, O te 'ihr', syrj. ti, tije, wotj. $t i \sim$ fi. $t e$, lp. dì, mord. tịn, tin.

338. TscherW tor, O tür, (CKr.) tṇr 'Scheide' $\sim$ fi. terä, lp. derre.

339. Tscher. $\beta i \cdot s ̌ t ə, \beta i \cdot s t \grave{\partial}$ 'Spelt, Dinkel', ? wotj. vaź, vał $\sim$ fi. vehnä, mord. viiš, viěsä.

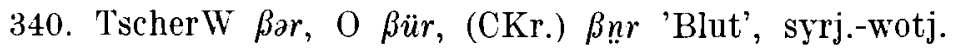
vir $\sim$ fi. veri, lp. vârrâ, mord. vér.

341. TscherW (K) Bär, (J) ßer, O Ber 'Stelle, Platz'; W

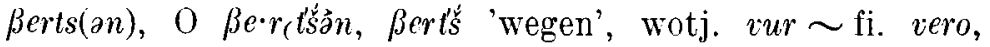
mordM varanc.

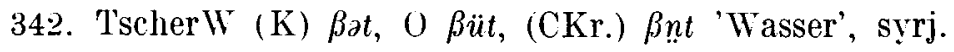
$v a$, wotj. $v u \sim$ fi. vesi, mord. ved.

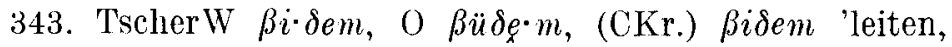
führen' fi. vetää, mordM v́clams, v́ct̀ms, E v́edams, v́ełams, vifims. Die Vertretung des Mordwinischen, insbesondere die des Mokschanischen, scheint auf urspr. ${ }^{*} \ddot{a}$ hinzuweisen.

344. 'T'scherW pə·laš, pə•lak $\chi_{s}$, palš, O (P B M) pâlâš, (CMU)

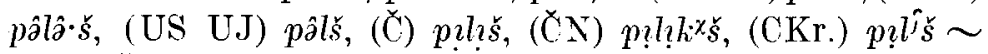

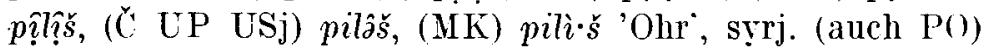
pel, wot,. pel $\sim$ lp. belllje, mord. pilte. - Lp., mord. < *peljä.

345. 'T'scherW (K) kel-, (J V) kal-, () kül-, (C CKr.) knl-, (UJ CČ J'T) kâl-, (UP) kui- 'müssen', syrj. (auch SO) kolni, PO kól-, wotj. kulk lp. gâllgât, mord. kel'jems.

346. Syrj. kertni, kertalni 'binden', wotj. lertini, kerttini, (Wied.) körtyny $\sim \operatorname{lpO}{ }^{*}$ gârltât $(=\operatorname{lpI}$ korìtå $\delta$. Lp. wahrscheinlich $<{ }^{*}$ kertte-.

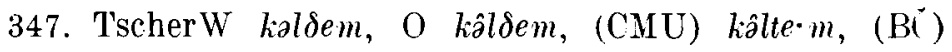
kâlde $\cdot m$, (CKr.) kị̂tem, (MK UP USj) kildem 'binden' $\sim \mathrm{lp.}$ galldet, mord. kildems.

348. 'Tscher. $\ddot{o} \cdot r \delta \partial \check{z}, \ddot{o} \cdot r \delta a z z$ 'Seite' (könnte dieses Wort eine Ableitung von $\mathrm{W}[\mathrm{K}] \ddot{a} \cdot r \delta \partial,[\mathrm{J} \mathrm{V}] \grave{e} \cdot r \delta \partial, \mathrm{O} \grave{e} \cdot r \delta \partial, e r \delta e \cdot, e \cdot r \delta e$, [UJ] ördö. 'Schenkel' sein?), syrj. (auch SO PO) ord-'Seite, Umgebung', ord-li 'Rippe', ordes 'die aufrechtstehenden Sei- 
tenbretter im Schlitten'; orde, $\mathrm{P}$ erde, PO ốrdo 'zu', wotj. urd 'Rippe', urdes 'Seite' $\sim$ lp. cerlte 'Seite, Rand (des Bootes, der Insel)', èrtig 'Seite (des Menschen, des Tieres)', mord. iŕles 'Rippe'. Im Mordwinischen die Urform *ertä-, im Lappischen *erttä. Die letztgenannte Variante beruht wahrscheinlich auf der sporadischen Gemination $t>t t$ nach $r$.

349. Syrj. (auch SO PO) mort 'Mensch, Mann', wotj. murt id. 〜 mord. miŕde, mârde 'Mann, Gatte'. - Die indirekte Zusammengehörigkeit der perm. und mord. Wörter ist seit alters angenommen worden; wahrscheinlich können sie aber auf eine gemeinsame Urform * mertä zurückgehen. Die Vertretung des in- bzw. auslautenden Konsonantismus ist dieselbe wie im Wortpaar syrj. nort $\sim$ mord. nurdo (141).

350. Tscher. šöroní, šörrońa 'Gold' mord. sịnne < ${ }^{*}$ serñä. Von derselben Urform kann vielleicht auch syrj.-wotj. zarni hergeleitet werden. (Dagegen setzen die entsprechenden Würter in den ugrischen Sprachen irgendeine hintervokalische Urform voraus.) Arische Lehnwörter.

351. Syrj. t'segni, PO cíg- 'brechen', wotj. t'sigini $\sim$ ? mord. sivems. Urform ?*'senä-, ?*t'senä-.

352. TscherW sar, O šâr 'Charakter, Art, Gewohnheit, Weise' syrj. śer.

353. Syrj. kil, kiv 'Zunge, Sprache, Rede, Wort, Stimme', wotj. kill, kịl $\sim$ fi. kieli, lp. giellâ, mordM licl', E kiel'.

354. Tscher. $l i \cdot \ddot{a} m$, lia $\cdot m$ 'sein, werden, möglich sein', syrj. (auch SO) loni, PO lus, wotj. luni, luini, lup̣̂? 'werden, sein; möglich sein'; med lo 'es sei! möge werden!', ug lo, uz lo 'kamn nicht sein, es geht nicht' $\sim$ fi. lie-, lp. let.

355. Tscher. lem 'Suppe, Bouillon', wotj. lim (syrj. lem) $\sim$ fi. liemi, lp. liebmâ, mordM $l \varepsilon \dot{m}$, E lem.

356. Sỵj. mil, miv, (PO) mol : m.-kid, -kod 'Verstand, Vernunft, Charakter', wotj. mị̂l, mị̂l-kị̂ $\sim$ fi. mieli, lp. miellâ, mord $M \dot{m} \varepsilon l$, E mel.

357. Tscher. ne·läm, nela'm 'schlucken, verschlingen', syrj.

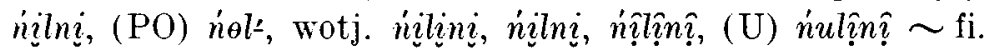
niellä, lp. njiellât, mord. niltems.

358. Tscher. pel, Bel 'Seite' $\sim$ fi. pieli 'Rand usw.', mordM $\dot{p} \varepsilon l$ ', E ṕpl' 'Seite'. 
359. Syrj. bel' 'Tür- od. Fensterpfosten' $\sim$ fi. pieli 'Pfahl. Pfosten, Pfeiler, Säule', mordM $\dot{p} \varepsilon l$ 'Stange, Zaunstange'. 360. TscherW šin, O šen, (CMU) šin 'Baumschwamm, Zunder' $\sim$ fi. sieni.

361. Tscher. ner 'Nase', syrj. nir, (PO) nor, wotj. nịr.

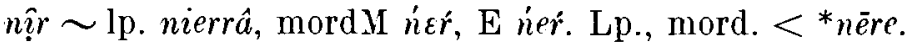

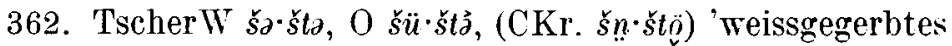
Leder; Riemen' fi. hihna, lpSchw. ${ }^{*}$ sâslte (= L sas̄ne; vorl. lp Nislte, K šišñe), mord. ǩšna, šna< balt., rgl. lit. šiǩšnà.

363. Syrj. šir 'Maus', wotj. šir, šị̂r $\sim$ fi. hiiri (<*šiner. vgl. FUF XXX 18), mord. tšejeŕ, tševeré, šejâr.

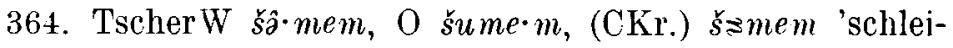
fen' fi. hioa, hivoa, lp. sâggjet, mord. tšsovams, šovams.

365. ? TscherW $i m z: i_{i}-\beta \partial_{0} t$ 'Wasser, das sich unter der Schwiele sammelt', syrj. $e \check{z}$ 'Hautteil des Felles (nicht Haare)' $\sim$ fi. iho, lp. âsse, mord. jož, jožă.

366. Tscher $i$, ii 'Jahr' $\sim$ fi. ikä, lp. jâkke 'Jahr' (âkke 'Alter' < fi.), mord. ije.

367. Syrj. jen, jenm-, I jen, PO jen 'Gott, Heiligenbild. Himmel', wotj. in, inm- $\sim$ fi. ilma, lp. âllbme.

368. SyrjPO nim- : nimás', ńimál- 'saugen' $\sim$ fi. imeä, lp. njâmmât.

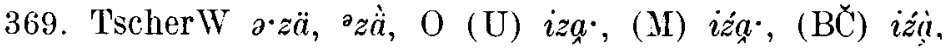
(CKr.) ?záa 'älterer Bruder; jüngerer Bruder des Vaters' fi. $i s \ddot{a}, \operatorname{lpK}{ }^{*} \hat{a} \check{c} c ̌ e\left(=\mathrm{Kld}\right.$. $\left.\dot{a} \bar{D} t t^{\prime}\right)$, ? mordM otśc.

370. ? Syrj. kośni 'schälen, abrinden, reissen', $\mathrm{PO}$ kúśstt-. wotj. keṣ́̂̂n $\sim$ fi. kishoa, lp. gâilkot (I kiškod).

371. Tscher. kiü, kïi 'Stein', syrj. $k i: i z-k i$, wotj. ke, (MU) kiö fi. kivi, mord. liev.

372. Syrj. moń, SO moń, PO mun' 'Schwiegertochter'; Le

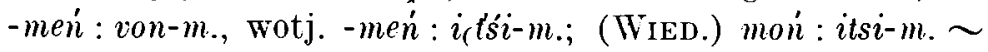
fi. minï̈, lp. mânnje.

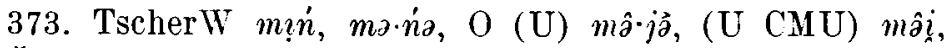

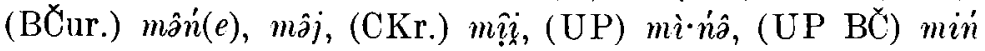
'ich', syrj. me, PO me, me, wotj. mon $\sim$ fi. minä. (Lp., mord. mon vertritt einen anderen Typus.)

374. TscherW lam, O lüm, (CKr.) lnm 'Name', syrj. nim, wotj. nim, nín $\sim$ fi. nimi, lp. nâmmâ, mord. l'em. 


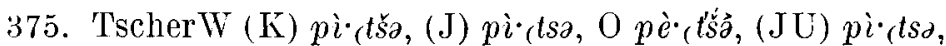
(CMU) pi· (t'se 'Zaun, Einzäunung, Gehege', svrj. (auch SO) potș, PO poč, wotj. putşs $\sim$ fi. piha.

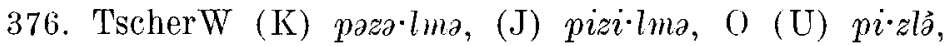

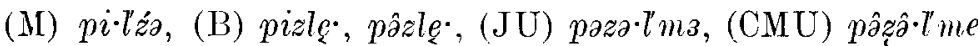
'Vogelbeere; Vogelbeerbaum', syrj. peliś, pelid'ź, wotj. paleś, paledź, (J M) påueź fi. pihlaja, mord. ṕizol, piźâl.

377. Syrj. peš 'Kienspanhalter', pešni 'einen Kienspan in den Halter stecken', PO ṕés- $\sim$ fi. pihti, lp. bâs|tha, mord. ṕěs, $p \check{s}^{\circ} t^{\prime} a \cdot m s$.

378. Tscher. pü, püi 'Zahn', syrj.-wotj. pin $\sim$ fi. pii (<* pine, s. FUF XXX 47-48), mord. ṕej, ṕev, ṕen.

379. TscherW pal, O (U CMU BČur.) pâl, (CKr.) p? l, (M) pil' 'Wolke', syrjU piv', I pi< ${ }^{*}$ pil, wotj. pil'em fi. pili i, lp. bâllvâ, mord. ṕejel', pel'.

380. Syrj. pemid, PO pimot 'finster; Finsternis', wotj. pelmit, penimit, peimît $\sim$ fi. pimeä.

381. Wotj. ped : p.-pal 'äussere Seite' fi. pinta.

382. TscherW pi $\check{z} \ddot{a} m$, O piža $m$, (B) pâžam 'sich auschliessen, kleben bleiben'; W pìžzlitem, O pižàlite $\cdot m$, (CMU B) pâzâłkte $\cdot m$ 'befestigen, anzünden' fi. pysyä, (dial.) pisyä; pysyttä̈, mord. peznems. - Eine alte Ableitung dieses Wortes kann tscherW (K) pi štęm, O pâšte $m$, (M) pište len, setzen, legen, hineinstecken' $\sim$ fi. pistä̈, lp. bâsltet sein. 383. Syrj. rinišs 'Getreidedarre, Riege', wotj. ṣ̌̂n? $r$, inšir $\sim$

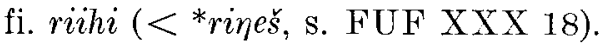

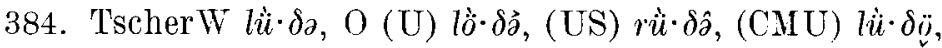
(B) rü $\delta \ddot{o}$. 'Falle (bes. für Mäuse)', syrj. ri fi. rita, lp $\mathrm{K}{ }^{*}$ râtte $\left(=\mathrm{T} r a_{i} t t e\right)$.

385. Syrj. siger, śiger 'Dachstuhl, Dachfirst, Gewölbe', wotj. śig fi. sii (<*'sine, s. FUF XXX 48 ).

386. TscherW šìlla 'Igel' $\sim$ fi. siiti, mord. śejel $\left(<{ }^{*}\right.$ śijel, s. FUF XXX 19).

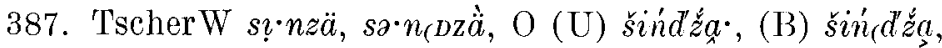

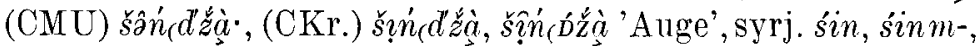
wotj. śin, śinm-, (U) śim $\sim$ fi. silmä, Ip. câllbme, mord. sel'me.

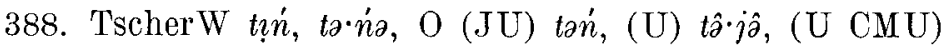




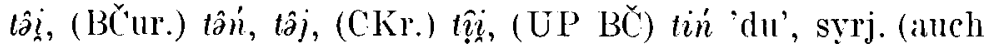
PO) te, wotj. ton $\sim$ fi. sinä $<{ }^{*}$ tinä. - Lp. ton, don, mord. ton vertreten einen anderen Typus.

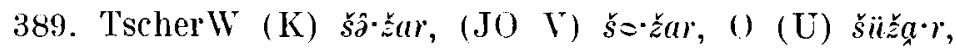

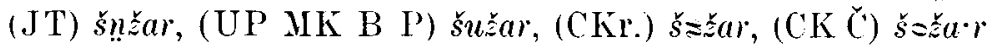
'jüngere Schwester', syrj. sozor, wotj. suzer $\sim$ fi. sisar, est. sõsar, mord. sazor, sazâr. - Fi. < balt.; tscher. < entweder balt. oder iran.; mord. und perm. wahrscheinlich $<$ iran.

390. Syrj. sit 'Kot, Dreck, Mist', wotj. sit' fi. sitta.

391. Syrj. vez (in einigen Ausdrücken) 'Neid, Begierde', vežęlting 'eifersüchtig sein', PO vižx liti-, wotj. vož 'Zorn, Grimm' fi. viha, lp. vâšše $<$ fi.

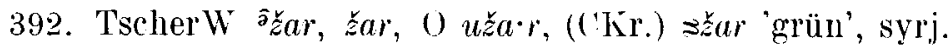
vež, $\mathrm{PO}$ viž 'grün, gelb', wotj. což $\sim$ fi. vihanta, viheriä, vihreä, mord. ažo. - Das Wort kann etymologisch desselben Ursprungs sein wie Nr.391.

393. Syrj. vez: tui-r., PO $v i z: t u j-v$. 'Wegkreuzung, Wegscheide' $\sim$ ? fi. rita 'schiefe Lage; schief'.

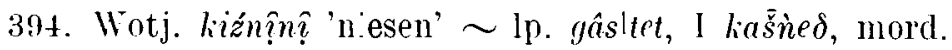
k'ešna-, kešne-, ǩšna-<*kiśnä-, *kišnä-.

395. ? TscherW ka škęm, O kâške $m$, (CKr.) kî̌kem, M kǐš$k e \cdot m$ 'werfen, schleudern; begiessen', syrj. kiśkyny, kiśkal-,

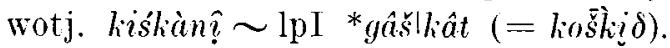

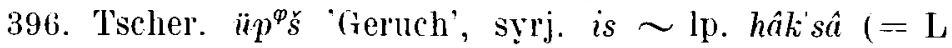
hapsa, I o $\left.\bar{p} \bar{s}^{A}\right)$, mord. op̀pś (ś).

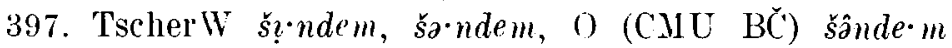
(BČur.) šândem, (CKr.) ṣ̌̂ndem 'stellen, legen' $\sim 1 p$. sâdldet 'spannen'.

398. 'Tschero kâre $m$, (CKr.) kîrem, (M) kirę $m$ 'schlagen (z.B. mit dem Stock, mit dem Hammer)', syrj. keralni, PO k'éral-, wotj. koràni $\sim$ mord. kérams $<{ }^{*}$ kirï̈-.

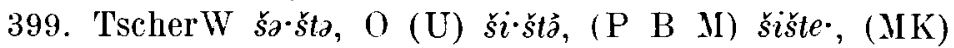

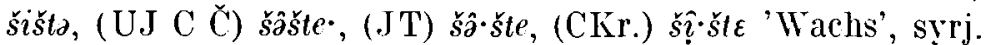
siś, siiśt-, P śiś, wotj. śuś, śus mord. kišta, šta.

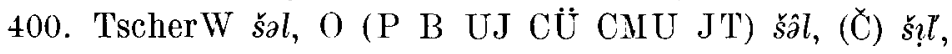
(CKr.) šll, (CK) šil, (MK UP) śil 'Fleisch', wotj. sil' 〜 ? mord. sịvel.

401. TscherW (K) $u \cdot r \delta e m,(\mathrm{~J}) \beta \diamond \cdot r \delta e m, O(\mathrm{JU}) \beta \approx r \delta e \cdot m$ 
'halten, unterhalten, ermähren, pflegen, erziehen', syrj. verdni, PO vérd-, wotj. cordini. - Einige Forscher haben vermutet, dass man hier ein indogerm. Lehnwort hätte, vgl. ai. $v r d h$ 'aufziehen', ar. vur $\delta$-.

402. TscherW š̀े ma, O š̀̀ $m \vec{a}$, (CMU) š̀ schwarz', syrj. sim 'Rost; dunkel'.

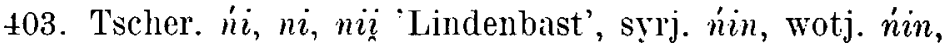
nin $\sim$ fi. nïni.

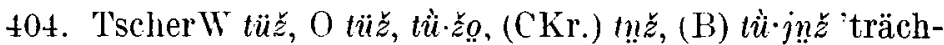
tig' $\sim$ fi. $\operatorname{tiine}(h)$.

405. Syrj. cainni herbeibringen, mitbringen, tragen, führen', wotj. vajini, vaini $\sim$ fi. viedä $\left(<^{*}\right.$ vi $\gamma e-$, s. FUF XXX

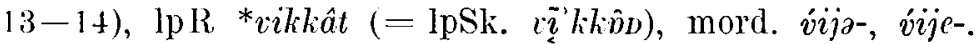

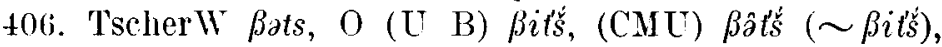
(CKr.) Pįt's 'fünf', syrj. vit, veti-min, wotj. vit' $\sim$ fi. viisi, lp.

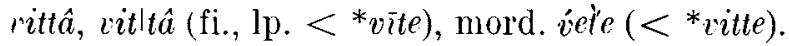

407. 'Tscher. rün 'Reif; Schnee auf den Bäumen' $\sim 1 p$. ridne.

408. TscherW $k \partial \cdot l m o, \mathrm{O}$ (U) $k \hat{a} \cdot l m \hat{\partial},(\mathrm{CMU}) k \hat{\partial} \cdot l m e$, (BCur.)

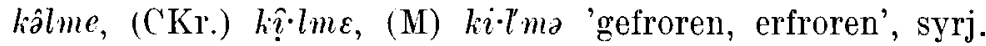
kin, wotj. lịn, lingm- fi. kylmä, lp. gâlmâs, mord. kelme.

409. TscherW (K) kats, (JO) knts, (V) küts, O (P BJp

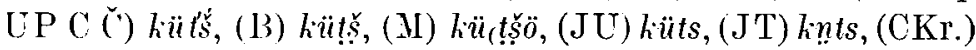

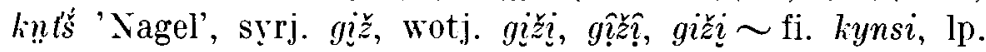
$g \hat{a} \tilde{z}^{\mid} \vec{z} \hat{a}$, mord. lienže.

410. TscherW (J) kn! $\cdot n^{d} z e m$, O künd ž́ $e^{\cdot} \cdot m$, (JU) künoze $\cdot m$ 'orraben, hervorgraben' $\sim$ fi. kyntiä.

411. Sỵrj. kiśmini 'reifen, reif werden', wotj. kiśmàni $\sim$ fi. kypsä, kypsyä, lp. gik'sât, *gâklšât $(=1$ kop̄ša $\delta)<\mathrm{fi}$.

412. Wotj. kịtkini, kị̂tkị̂? 'anspannen' $\sim$ fi. kytkeä.

413. Wotj. kii , kịi 'Schlange' fi. kyy (<*küi, *küje, s. FUF XXX 48), mord. kuj, kijov.

414. Wotj. kìli, kil : śin-k. 'Träne' fi. kyynel, lp. gânjâl $(<*$ künel $)$.

415. TscherW kə•ńer, O (U CMU) kâne•r, (BČur.) kâner,

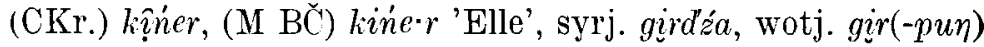

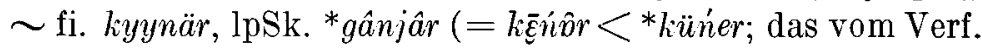
FUF XXX 16 erwähnte 1pI kơnąur ist irrtümlich), mord. kenér. 
416. SyrjI lō $\left(<* l_{o} l\right)$ 'Kern-, Herzholz' $\sim$ fi. lyly.

417. Syrj. miďz 'Stütze' fi. nyde, mord. neže.

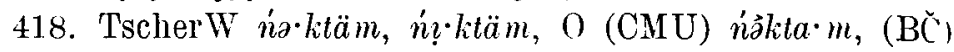
n’âkta.m, (CKr.) ń?̣̂ta $m$ 'schinden' $\sim$ fi. nyhtää, mord. nertams, neftams.

419. Wotj. pižni, pq̣žni 'Schlinge, Dohne' fi. pynnä.

420. TscherW šüm, O šüm, (CKr.) šn m 'Herz', syrj. śelem. PO śốlom, wotj. śulem, śuuem fi. sydän, lpSk. *čâde (i.

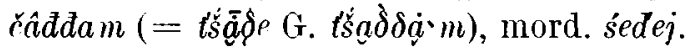

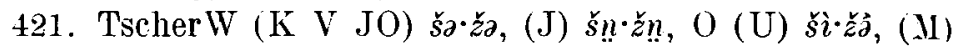

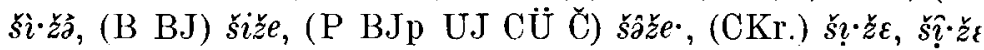

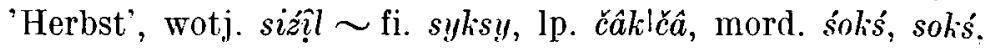
śoks.

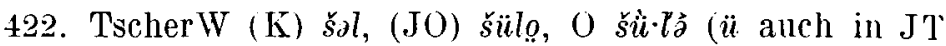
CKr.) 'Klafter', syrj. sill, siv, wotjG sị̂, U sul, J II sul fi. sỵli, lp. sâllâ, mord. sęl, sèl.

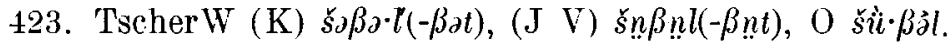
(CKr.) šn $\beta n \cdot l(-\beta n t)$ 'Speichel', syrj. ślalni, PO súlal-, wotj. śalanị, śal fi. sylki, syllieä, lp. čollgâ, čollgât, mord. śel'ge. sel'gems.

424. Syrj. sodni, SO sodni, PO súd- 'sich vermehren, zınehmen' $\sim$ fi. syntyä, lp. šâdldât $<$ fi.

425. Tscher. šör 'Seite, Kante, Rand' $\sim$ fi. syrjä, mord. tšire, tšire, sire, šiř

426. 'Tscher. šü, šü 'Kohle' fi. sysi, lp. čâddâ, mord. śed'. 427. Tscher W ša šk $k \ddot{a} m$, O šüška $m$, (CKr.) šṇškam 'stopfen. buttern' $\sim$ fi. sysätä, est. süskama, lp. sâsık̂t, sâslket 'rub oneself against; move up and down (like the male reindeer' when copulating); jump on to and mate with; have sexual intercourse with, fuck (obj.: the woman)'.

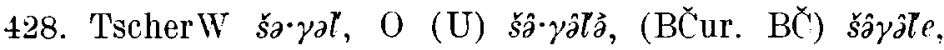

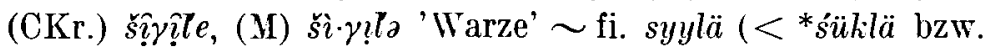
*śüplä), lp. čiwlhle, mord. śilge, śelge.

429. TscherO lï·m’̀ 'Leim', syrj. lem, PO lémet-, wotj.

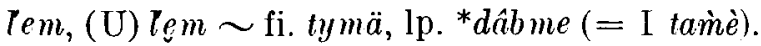

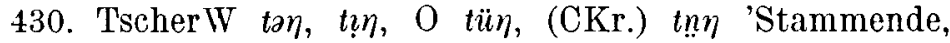
dickes Ende eines Baumes; Anfang', syrj. din, (P) din, wotj. $d i n, d i \eta,(\mathrm{G}) d i \dot{n} \sim$ fi. tyvi, mord. $t \epsilon-, t e j-$. 
431. 'TscherW Bim, O Bem, (CIU) $\beta i \cdot m e$ 'das Mark', syrj. vem, $\mathrm{PO}$ vim, wotj. rim, viim, vijim $\sim$ fi. ydin, lp. âdâ G. iddâm(â), mord. udime, uderie.

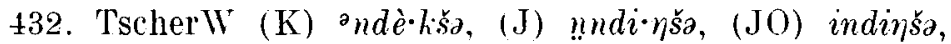

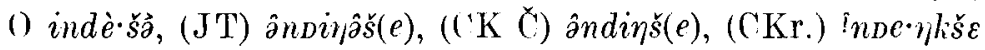
'neun', vgl. syrj. ek-mis, (I) ok-mis, wotj. ukmis fi. yhdcksän, Ip. olilce, $\mathrm{lp} \mathrm{R} * \hat{a} l_{i} \mid c e$ (= T alice).

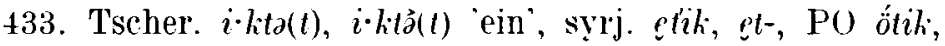
wotj. odig, og fi. ylisi, lp. okltâa, âkltâ, mord. v́e, v́vjle.

434. TscherW (K) $\beta a l-: \beta \partial \cdot \ln \alpha$, O (CMU) $\beta \ddot{u ̈ l-: \beta u ̈ l n o ̈, ~}(\mathrm{~J} T)$

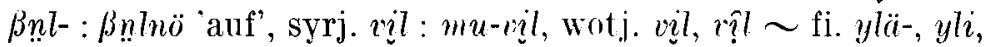
lp. âlle-, âlâ, mord. vellks.

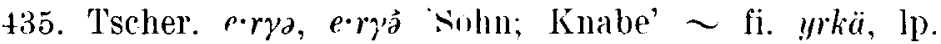
irlge $<$ fi.

436. Syrj. voi, (SO) oi, PO yj 'Nacht', wotj. ui, ui $\sim$ fi. yö $(<* \bar{o}<* \ddot{u} j e-$, s. FUF XXX 39), lp. iggjâ, mord. ve.

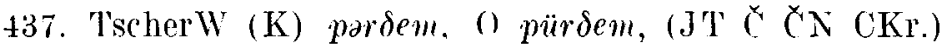

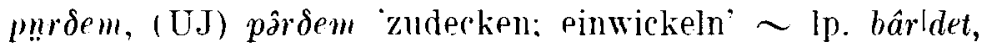
horldet.

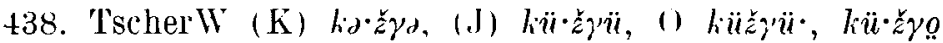
(ii auch in CK CKr. Č) 'dick', syrj. kiz, wotj. kîz 〜 Ip. gâssâg.

439. TscherO (U) lüs, (M) lü·ins' 'Tangel, Nadel; Tannennd. Fichtenzweig', syrj. lis, (V (') lish-, (P) ris, wotj. lis.

440. 'TscherO ( U M) lüšté 'm' 'melken', syrjl liśtint.

441. 'Tscher() mükth, mültö 'Fobio fluviatilis', (nach einer anderen Angabe:) 'wahrsch. Lenciscus alburmus', syrj. mik.

442. 'TscherW (K) s.orta, ( ) š̈ larn. Zwirn', syrj. šert, P() šörl, wotj. šort.

DER TSCHEREMISALG 'HE VOKAIISMUS.

1. Wo a.

Hierfür gibt es ungefähr 30 Beispiele. Die Angabe Wıcıuxxis, dass es etwa 50 gebe (FUF XVI, Anz. 45), kann 
nicht zutreffen. Zu den Beispielen, die Beke, Cs. Ny. 30-31,

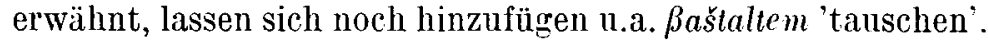
kaklaka 'krumm', kaño 'ähnlich', lakem 'stecken bleiben', laptşâk 'Stückchen, Fetzen', latš 'pünktlich', marô, marị 'Ehemann, Tscheremisse', namam. 'essen (Kinderwort)', raškaltem 'einschlagen (vom Blitze)' (< raškem 'knallen, knirschen'), tšal 'dicht'. In jungen Lehnwörtern, namentlich tatarischen, ist $\mathrm{W} O a$ sehr gewöhnlich.

In dieser Gruppe hat schon das Urtscheremissische a gehabt. Für kein einziges der ursprünglichen tscheremissischen Wörter, in denen wir urtscher. a antreffen, hat sich eine unbestreitbare finnisch-ugrische Etymologie nachweisen lassen. Z.B. die Entsprechungen, die Wichmann FUF XVI, Anz. 40-41 für sechs tscheremissische $a$-Würter bringt. sind alle, wenn nicht zu verwerfen, so doch sehr unsicher.

In einigen Wörtern erscheint im Osttscheremissischen der interdialektale $a \sim u$-Wechsel, vgl. Wichmann, FUF XVI, An\%. 40, Beke, FUF XXIII $74-75$. Von diesen sind vortscheremissischen Ursprungs wahrscheinlich nur paremam ( $\mathrm{I} \mathrm{B} \mathrm{o)} \mathrm{'genesen'} \mathrm{(34);} \mathrm{\beta aštar} \mathrm{(} \mathrm{M} \mathrm{MM} \mathrm{JT} \mathrm{o)} \mathrm{'Ahorn'} \sim \mathrm{fj}$ ra(a)htera, mord. uk:štor (49); : Baštareš (If o) 'entgeren' (57). Beke scheint (i.a.O.) der Auffassung zuzuneigen, dass in den Wörtern, die ein derartiges lautliches Schwanken aufweisen, das $o$ sich aus dem häufiger anzutreffenden $a$ entwickelt hat. Das würden u.a. die mundartlich als o-Formen auftretenden späten Lehnwörter beweisen, deren russische und tatarische Originale a haben. Es fragt sich jedoch, ob man auf diesem Umstand viel aufbanen kann, denn beispielsweise in finnischen Dialekten zeigen junge, aus dem Russischen und Schwedischen übernommene Lehnwörter oft die merkwürdigsteı phonetischen Schwankungen. Ausserdem muss beim Vergleichen solcher Dialektwörter stets die Möglichkeit getrennter Entlehnungen und getrennter Lautsubstitutionen berïcksichtigt werden. Einige der von Beke erwähnten russischen Lehnwörter sind ihrer Struktur nach solche, dass es angebracht ist, in Bezug auf sie eine Bemerkung ron Räsäsw zu zitieren: "Beiläufig gesagt ist der Lautwert des russ. " und $a$ vor Akzent identisch in dortigen Mundarten. etwa $\hat{a}$. 
ein reduzierter IIinterzungen rokal" ('Tschuw. Lehnw. 79). Unter diesen Umständen befremdet das Schwanken nicht. Nach Ansicht des Verfassers gehören paremam, Baštar und die übrigen hier erwähnten ursprünglichen tscheremissischen Wörter zu dem Typ W $a \sim 0$, der weiter unten in Punkt 2 besprochen werden soll; in diesem Fall dürften also die im Osttscheremissischen in einem beschränkten Gebiet rorkommenden poremam, ßoštar usw. eine ältere Entwicklungsstufe vertreten als die Dialektformen, deren erste Silbe das der Vertretung im Westtscheremissischen entsprechende $a$ aufweist. Dieser Gedanke ist schon deshalb natürlich, weil der Vokal der ersten Silbe in all diesen Wörtern auf vortscheremissisches * $a$ zurückgeht, ganz ebenso wie beim Typ Wa $\mathrm{O} o$ (allerdings ist bei ßoštar auf Glund des mord. ukštor auch dic Ausgangsform * volštar möglich, vgl. FUF XXIX 233).

Wir gelangen also 71 dem Schluss, dass es sich beim Auftreten von urtscher. $a$ in solchen Wörtern, deren Gescr ichte in vortscheremissische Zeit zurückreicht, um eine zr.fällige oder sekundäre Erscheinung handelt. Im allgemeinen beschränkt sich das a auf die späte Wortschicht, deren Hauptteil deutlich deskriptive und onomatopoietische Wörter wie auch Lehnwörter bilden. (Ifenbar ist das ganze $a$ eine e r s t i m Späturtscheremissischen entstand e $n$ e Voka lkat e gor i e. Da es somit auf rein tscheremissischem Boden entstanden ist, hat es keine Bedentung für die vergleichende finnisch-ugrische Vokalgeschichte.

tuf die Frage, welche Gründe es veranlasst haben, dass ein derartiger Laut im Vokalismus der ersten Silbe in Crtscheremissischen erschien, kann man nur mit Jutmassungen antworten. Von Bedeutung dürfte der Umstand gewesen sein. dass, obwohl vortscher. ${ }^{*} a$ im Urtscheremissischen in der ersten Silbe regelmässig geschlossener wurde $\left({ }^{*} a>{ }^{*} \boldsymbol{i}\right.$ und daraus zuweilen weiter $*^{*}$. s. weiter unten), trotzdem das a nicht ganz aus dem Vokalsystem der Sprache rerschwand, denn in anderen als der ersten Silbe erhielt es sich unter bestimmten Bedingungen, rol. FUF XXIX 233, 239. In diesen Silben war es in mancherlei Flexionsendungen und Suffixen 
sehr häufig. Von da kann es auch in die erste Silbe von neuem eingeführt worden sein; $a$ ist ja ausserdem seinen phonetischen Eigenschaften nach eine Art Grundvokal, bei dessen Artikulation die Zunge der absoluten Ruhelage näher ist als bei den übrigen Vokalen, so dass wir sagen können, es besitzt in aussergewöhnlichem Mass natürliche Expansionsvoraussetzungen. Auffallend zahlreich sind unter den tscheremissischen $a$-Wörtern solche, die auch in der zweiten Silbe ein zum Stamm gehörendes a haben: ßaštar, ßaštareš, ßaštaltem,

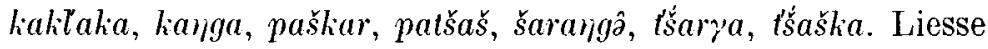
es sich denken, dass gerade zuerst in Wörtern dieses Typs, in denen das $a$ der zweiten Silbe assimilierend wirken konnte, im Späturtscheremissischen in der ersten silbe a erschienen ist? In ihnen war der Vokal der ersten Silbe, als den man am zwanglosesten $*_{a}^{a}$ annehmen kömnte, sogar noch $1 \mathrm{n} \mathrm{b}$ et o nt, während der Hauptton meistens auf jenem a der zweiten Silbe lag, eine Eigenschaft, die natürlich dessen assimilierende Kraft erhöhte. Nachdem das a auf diese Weise durch eine sporadische Assimilationserscheinung entstanden war, kanu es dann auch in Wörtern anderen Typs Fuss gefasst haben (vgl. auch später S. 207). (Ohne die durch tatarischen und russischen Einfluss eingeführte Lehnwortschicht wäre die Frequenz desselben in der Sprache jedenfalls sehr rering geblieben, denn auf den alten eigenen Wortbestand erstreckte sich seine Expansionsfähigkeit nicht.

\section{2. $W a \sim 0 o$.}

Beispiele gibt es', hierfür vielleicht 120 , von denen ВекE ('s. Ny. 33-38 etwa 75 erwähnt. Ausser diesen haben wir: ßotškầ 'Zuber', ßozem 'Lindenbast abschälen', ßožâlam 'sich

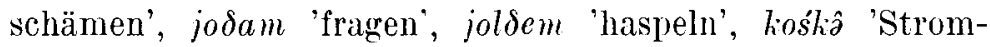
schnelle; schnell, reissend (Fluss)', kotama 'ein Fisch', kozâra 'rauh, grob', kožlanem 'auftrocknen', loßal 'Brachs', lo $\delta a k$ 'Scheide', loyâ 'ein Vogel (Seidenschwanz?)', lomâžam 'brüllen', lon'd’̌̉ổ 'Zwischenraum, Schicht', lotšâ 'Kind, das spät zu gehen anfängt', ložaš 'Mehl', nolâ 'Baumsaft, nota 'absicht- 
lich', notška 'feucht', ondak 'früh', rond'źem 'ausfasern, auswickeln', rož 'Loch', šoprengô 'Häher', šopš 'Weberspule', toßar 'Axt', toßa 'zottige Wolle, verfitzte Hanfstopfe', tolašm 'sich bemühen', tora 'weit, fern', torem 'wegrücken, zerstüren', totâ 'ein Fisch', tot'šem 'streben, sich bemühen', t'ššstra 'grob, rauh'. In tschuwassischen Lehnwörtern ist dieses Lautverhältnis sehr häufig. Beispiele gibt es mnter ihnen über 100 , also ungefähr ebensoviel wie eigene Wörter vleichen Typs.

Die Forscher sind sich nicht darüber einig geworden, auf welche urtscher. Ausgangsform das Lantverhältnis Wa 0) o zurückgeht. Gombocz (NyK XXXIX 249), Wiснwaxy (FUF XVI, Anz. 39) und Stemitz (Vok. 95) sind der Meinung, dass sie ein $a$ gewesen ist, während man nach Räsävex (Tschuw. Lehnw. 79) und Beke (FUF XXIII 67) ron einem o ausgehen muss. Wir betrachten zuerst die Begründungen der letztgenannten Forscher. Räsänen macht u.a. darauf aufmerksam, dass es im Tscheremissischen viele tschuwassische Lehnwörter gibt, in denen wir das Lautverhältnis $\mathrm{W} a \sim \mathrm{O}_{o}$ finden, obwohl in ihren Originalen niemals ein a vorgekommen ist, z. B. W šala zaj, O šola 'link'< < tschuw. solaraj $\sim$ kas.-tat. sul, kirg. solakaj. Beke richtet sein Augenmerk in erster Linie auf solche westtscheremissische Mundarten, in denen gemäss der Vertretung im Osttscheremissischen in zahlreichen sowohl eigenen als auch aus dem Tschuwassischen entlehnten Wörtern ein $o$ erscheint. Eine derartige Vertretung weisen die von ihm erforschten JO- und V-Dialekte auf (über diese s. näher FUF XXII 93,

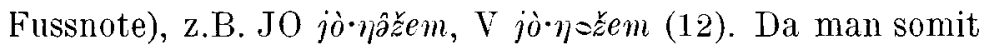
auch auf westtscheremissischem Gebiet ein 0 antrifft, sei das ein Beweis für die Ursprünglichkeit des $o$ im Vergleich zum $a$. Nun muss man aber fragen: Woher kommt es, dass auch jene westtscheremissischen Dialekte, in denen der von Beke angenommene Wandel $o>a$ stattgefunden hat, eine grosse Menge von Wörtern haben, in deren erster Silbe das o sich erhalten hat? Diese Frage kann hier unmöglich übergangen werden. Und zweitens: Die Vertretung $\mathrm{W} a \sim \mathrm{O}$ o steht deutlich an erster Stelle in solchen Wörtern, bei denen der Vokal der 
ersten Silbe auf rortscher. ${ }^{*} a$ zurückgeht. Wesentlich weniger Fälle gribt es, in denen man von vortscher. ${ }^{*} o$ ausgehen muss. Dieses Lautverhältnis wird durch folgendes Material bewiesen:

a) Auf vortscher. * $a$ geht $\mathrm{W} a \sim \mathrm{O}_{0}$ zurück in den Wör-

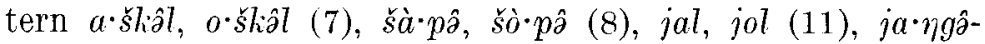
žem, jònàž

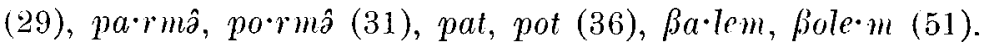

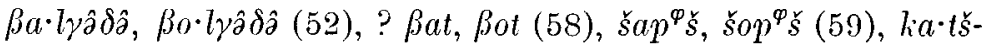

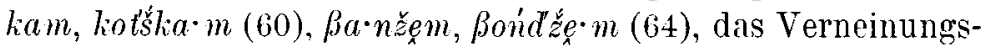

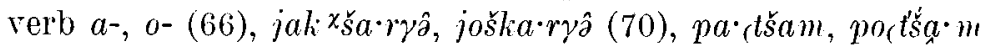

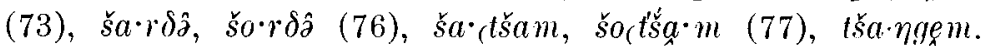
t'̌́one $\cdot m$ (78). Auch tscherW $W^{\top} a \cdot k s \hat{a}$ (23) gehört vermutlich zu diesem Typ, obwohl man das nicht sicher feststellen kann. weil keine osttscheremissische Entsprechung dafür beigebracht worden ist. Insgesamt also 19-21 Fälle.

b) Ausser den obigen Beispielen haben wir Veranlassung. die folgenden zum Typ Wa O o gehörenden Wörter zu erwähnen, bei denen man die ursprüngliche Qualität des Vokals der ersten Silbe nicht immer mit Sicherheit feststellen kann, in erster Linie deshalb, weil sie entweder keine Entsprechungen in den westlicheren finnisch-ugrischen sprachen haben oder diese Entsprechungen selbst die Möglichkeit zu verschiedenen Deutungen geben. Gewöhnlich jedoch haben diese Wörter Entsprechungen in den permischen Sprachen und wahrscheinlich ist in beiden Sprachgruppen gerade rortscher. *a als Ausgangsform des Vokals der ersten Silbe anzusetzen.

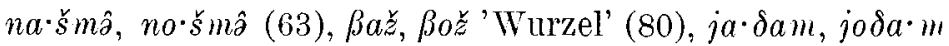

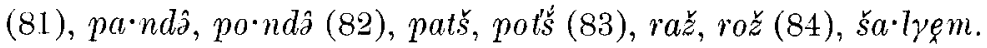

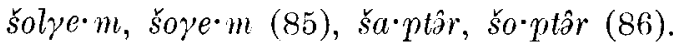

c) Vortscher. ${ }^{*}$ wird durch $\mathrm{W} a \sim 0_{0}$ vertreten in den

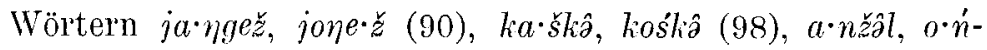

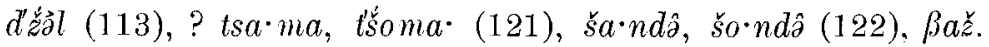
ßoz 'Verzweigung' (148). Also nur 5-6 Beispiele. - Im allgemeinen hat vortscher. ${ }^{*} o$ im Tscheremissischen eine ganz. andere Vertretung, nämlich $\mathrm{W} \cap o$ oder $\mathrm{WO} u$. s. unten SS. 250-251. 
d) Ganz gelegentliche vereinzelte Fälle treffen wir an, in denen $\mathrm{W} a \sim 0$ o auf $* u$ zurückgeht: ? ka·ja, koja (184), $\beta a \cdot \delta a r, \beta o \delta a \cdot r(210)$, also $1-2$ Beispiele. Hat sich das * $u$ unter dem assimilierenden Einfluss des hauptbetonten $a$ der zweiten Silbe sporadisch geöffnet?

e) Für das Zurückgehen des erwähnten Lautverhältnisses auf $*_{\bar{o}}$ gibt es ein einziges unsicheres Beispiel: $\check{s}_{a} i_{n}-$, šoin- (160).

Unsere Beispiele zeigen, dass $\mathrm{W} a \sim \mathrm{O}_{o} \mathrm{n} \mathrm{u} \mathrm{r}$ di e la u tgesetzliche Vertretung ron vortscher. *a ist. Wenn irgendein anderer vortscher. Vokal im Tscheremissischen auf diese Weise vertreten ist, handelt es sich um eine sporadische Entwicklung. Wie wir weiter unten (S. 193) sehen werden, gibt es recht zahlreiche Fälle, in denen vortscher. ${ }^{*} a>$ W O o; da handelt es sich zweifellos um ein schon urtscheremissisches 0 . Wichtig ist aber, dass urtscher. o, anders als das Lautverhältnis $\mathrm{W} a \sim \mathrm{O}_{\mathrm{o}}$, ganz ebensooft auch auf vortscher. ${ }^{*}$ zuräckgeht. Gerade die Vertretung des urspr. ${ }^{*}$ im Tscheremissischen berechtigt zu dem Schluss, dass sich das Lautverhältnis $\mathrm{W} a \sim \mathrm{O} o \mathrm{nicht}$ a us u r t s c h er. o e n t w i c k e lt h a t. Wir können also Räsänen und Beke nicht beipflichten.

Den ebenerwähnten grundsätzlichen Unterschied zwischen der Entwicklung der ursprünglichen ${ }^{*} a$ und ${ }^{*} o$ bemerkten schon Gombocz und Wichmann, aber auch ihre Auffassung. nach der also $\mathrm{W} a \sim \mathrm{O} o<$ urtscher. $a$, kann nicht richtigr sein. Obwohl es nicht viele Wörter vom Typ W O a gibt, der oben in Punkt 1 behandelt worden ist, zeigen sie doch unbestreitbar, dass zum Vokalismus der ersten Silbe im Späturtscheremissischen auch die Lautkategorie a gehörte, die sich im ganzen Sprachgebiet unverändert erhalten hat. Nach Wichmann müsste man annehmen, dass es in Urtscheremissischen einen Vokalstufenwechsel $a \sim o$ gregeben hätte, aus dem in den Ostdialekten durch Reihenübergang im grössten Teil der urtscher. $a$-Wörter ein $o$ geworden wäre. Diese Erklärung ist jedoch bereits völlig veraltet und ebenso unmöglich wäre die Annahme eines sporadischen Wandels $a>0$ im Osttscheremissischen. weil ein derartiger Wandel in den älteren eigenen Wörtern der Sprache und in den aus dem Tschuwassischen ins Urtschere- 
missische entlehnten Würtern eingetreten wäre, aber die junge deskriptiv-onomatopoietische Wortschicht unberührt gelassen hätte.

Das Problem wird gelöst durch die Annahme, dass hinter lem Lautverhältnis $W^{r} \sim \mathrm{O}_{o}$ urtscher. ${ }^{*} a$ stelıt, mit anderen Worten, ein labialisierter und möglicherweise etwas geschlossener a-Vokal, der sich dann in den Westdialekten zu $a$ und in den Ostdialekten zu $o$ entwickelt hat, wobei jedoch in einigen Wörtern auch im Westtscheremissischen mundartlich die $o$-Vertretung anzutreffen ist. Die letztgenannte Erscheinung muss irgendeine alte Einwirkung der (1)stmundarten zur Ursache haben.

Die Annahme eines $a$ als Vorgänger von tscherW $a \sim 0$ " ist eigentlich nichts Neues, denn schon Gombocz (NyK XXXIX 252) und früher auch Beke (Cs. Xy. 38) gingen von dieser Urform aus. Der Unterschied besteht nur darin, dass sie im Anschluss an Setäläs Vokaltheorie annahmen, dieses $\stackrel{i}{a}$ stamme aus der fi.-ugr. Ursprache. Aber im Mordwinischen hat sich ja das illabiale $a$ erhalten und ebenso beweisen die recht zahlreichen Fälle im Lappischen, in denen das fi.-ugr. ${ }^{*} a$ durch ein illabiales sog. helles $a$ vertreten wird, dass erst im Urtscheremissischen die Entwicklung ${ }^{*} a>*_{a}^{\circ}$ stattgefunden hat. In den wolgatürkischen Sprachen sehen wir eine interessante Parallelerscheinung: Die Labialisierung des urtürkischen a trifft man auch dort verschiedentlich an, u.a. im Baschkirischen (a) und Özbekischen (ein offener $o$-Vokal), vgl. RÄSÄxEx Materialien zur Lautgeschichte der türkischen Sprachen 82-83 mit Lit. Nach Räsänen kann auch im Tschuwassischen zur Zeit der tschuwassisch-tscheremissischen Berührungen das urtürkische $a$ in der ersten Silbe durch $\stackrel{a}{a}$ vertreten gewesen sein (Tschuw. Lehnw. 81). Wenn wir uns daran erinnern, dass das Lautverhältnis $\mathrm{W} a \sim \mathrm{O}_{0}$ in den tschuwassischen Lehnwörtern des Tscheremissischen sehr häufig ist, so ist es wahrscheinlich, dass es in beiden Sprachen gleichzeitig ein å gegeben hat. Vielleicht beruht dieses im T'scheremissischen geradezu auf einer Einwirkung der türkischen Phonetik. Zwingend ist jedoch diese Erklärung sicherlich nicht. 
Wie auch immer die von Räsänen angeführten Fälle zu erklären sein mögen, in denen türk. $o$ im Tscheremissischen in die $*_{a}^{*}$-Reihe übergegangen ist, grundsätzlich ist das keine andere Erscheinung als die entsprechende Entwicklung, die auch beim vortscher. ${ }^{*} o$ sporadisch auftritt.

Beke zählt FUF XXIII 71 sieben Wörter fi.-ugr. Ursprungs auf, bei denen in einigen osttscheremissischen C- und C-Mundarten statt des erwartungsgemässen $o$ ein $a$ steht, z.B. $\check{C}$ $\beta a \delta a \cdot r$ 'Euter', šar $\delta e$ ' 'Elentier'. Da sich diese Dialekte im Westteil des osttscheremissischen Sprachgebiets befinden. ist es wahrscheinlich, dass dies $a$ auf westtscheremissischem Einfluss beruht. Natürlich kann man sich auch einen sporadischen Wandel $\stackrel{\circ}{a}>a$ denken; in diesem Fall wïre das $a$ der C- und Č-Dialekte seinem Ursprung nach vom westtscheremissischen $a$ völlig verschieden.

\section{W 00 .}

Beispiele gibt es hierfür etwa 80 , bei Beke Cs. Ny. 38-41 etwa 60. Ausser diesen seien erwähnt jongẩ 'geräumig, frei'. kot 'Jahr, Zeit', lokšŝnd'žam 'behanen', lonam 'wannen, worfeln', loryem 'brodeln', oß̂̊ 'Schwiegervater', omâ 'Schlaf. Traum', omdâktem 'das Kalb an der Kuh saugen lassen'.

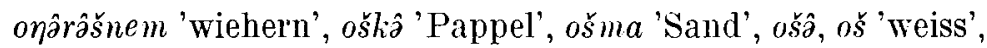
ožâ 'Hengst', pomâžaltam 'aufwachen', toreš 'quer', toš 'Rücken des Messers oder der Axt', t'śolyâzzam 'blinken, glänzen'. In den tschuwassischen Lehnwörtern kommt $\mathrm{WO}$ o wenigstens 20mal ror.

Schon im Urtscheremissischen hat es in den erwähnten Wörtern ein o gegeben, das sich also sowohl in den West- wie in den Ostdialekten erhalten hat. Dieser Laut ist heterogenen Ursprungs; er geht sowohl auf ursprüngliches * $a$ als auch auf ursprüngliches ${ }^{*} o$ und in geringerem Mass noch auf einige andere Vokale zurück.

a) $o<$ vortscher. ${ }^{*} a: \grave{o} \cdot \beta \hat{\partial}(6), k o \delta e \cdot m$ (14), kok, ko ktât

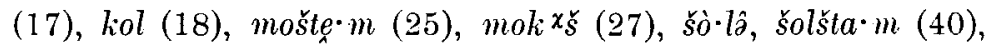

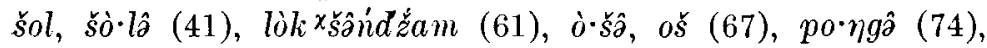
to $\bullet$ štâ (79). Beispiele hierfür gibt es 12 . 
Es seien auch die folgenden Wörter erwähnt, in denen im ()sttscheremissischen $0<$ vortscher. * $a$. Vorläufig ist es jedoch unmöglich, zu entscheiden, ob man bei ihnen ein urtscher. o oder * $a$ annehmen muss, weil sie in den west tscheremissischen Mundarten nicht festgestellt sind.

: O oktâs̆ (1), O fotkem (65), O moštem (72).

Nun stehen wir vor der Frage: Gibt es irgendeine Grundlage für die Entwicklung des ${ }^{*} a$ im Urtscheremissischen einerseits $\mathrm{zu} * \vec{a}$ ( $\mathrm{vgl}$. oben S. 192), anderseits zu 0 ? Offenbar lässt sich kein Lautgesetz als Crsache dieser Zweiteilung nachweisen. Wenn wir die $o$-Wörter nach dem Konsonanten vor oder nach dem Vokal gruppieren, sehen wir, dass in entsprechender Konsonantenumgebung auch *a-Vertretung vorkommt. Der einzige Unterschied, der möglicherweise von Bedeutung ist, ist der, lass in keinem einzigen $o$-Wort ein zum Stanm gehörendes $a$ in der zweiten Silbe steht, während dieser Typ unter den $* a$-Wörtern recht gewöhnlich ist. Licht in dieses Problem dürften jedoch die Frequenzverhïltnisse der beiden Vertretungsweisen bringen: Für die Entwicklung vortscher. ${ }^{*} a>$ urtscher. ${ }^{*} a$ gibt es im ganzen etwa 30 wahrscheinliche Beispiele, während die Fälle von ${ }^{*} a>o$ höchstens auf die Hälfte dieser Zahl geschätzt werden können. Es scheint, dass die $o$-Vertretung auf einer sporadischen Entwicklung $*_{a}^{a}>o$ beruht, die unter bestimmten günstigen phonetischen Voraussetzungen wohl stattgefunden hat. Dass ein Labialkonsonant vor oder nach den Vokal (die Fälle

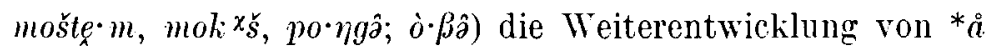
zu $o$ hat fördern können, ist klar. In den Wörtern $k o \delta e \cdot m$,

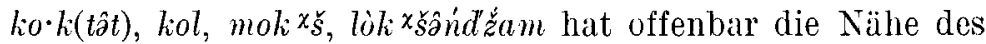
hinteren $k$ zu dem Wandel $*_{a}^{a}>o$ beigetragen, natürlich nicht als labialisierender Faktor, sondern als einer, der das Verharren der Zunge in zurückgezogener Stellung begünstigt hat. Besonders ein -hrs- nach dem Vokal scheint in dieser Weise gewirkt zu haben; später werden wir nämlich dasselbe beim urtscher. $u$ bemerken. Vielleicht ist hier der Hinweis am Platz, dass auch in dem Wort $\grave{o} \cdot s ̌ \hat{a}$, os ursprünglich die Verbindung $-k$ š - gestanden hat.

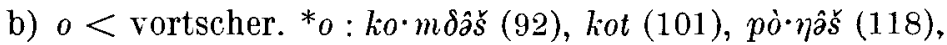




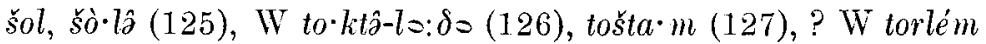

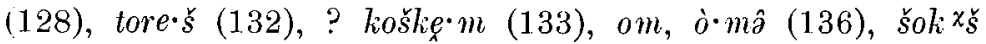
(137), šon (144), ? šož (145), o.šliâ (147). Also 11-14 Beispiele.

Diesen kann man noch mit ziemlicher Sicherheit O kordem (96) hinzufügen. Obwohl dieses Wort im Westtscheremissischen nicht festgestellt worden ist, ist es nicht willkürlich, darin ein urtscher. ${ }_{0}$ anzunelımen, wenn man in Betracht zieht, wie selten ursprüngliches $*_{0}$ sich zu urtscher. ${ }^{*} a$ entwickelt hat.

In diesen Fällen hat sich somit ursprüngliches *o im Urtscheremissischen unverändert erhalten.

c) Ein paar Wörter, deren urtscher. o entweder auf $*_{a}$ oder auf $*_{o}$ zurückgeht, wobei jedoch $*_{o}$ wahrscheinlicher ist:

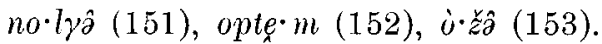

d) $0<$ vortscher. ${ }^{*} \bar{o} .2-4$ Beispiele: kole $\cdot m$ (155), ? kož (156), lo·mbà (164), ? lion (239).

e) $o<$ vortscher. ${ }^{*} \bar{u} .1-3$ Beispiele: kola $m$ (233), ?kon (239), ? loz (156). Hinsichtlich kon vgl. S. 302.

f) $o<$ vortscher. ${ }^{*} u$. Möglicherweise gibt es hierfür 2 Beispiele: tola $m$ (206), ton, tom (207). Falls man in diesen Wörtern wirklich auch für das Tscheremissische von ${ }^{*} u$ ausgehen muss, hat eine gelegentliche Vermischung mit der ${ }^{*} o_{-}$oder ${ }^{*} \bar{u}$-Reihe stattgefunden. Eine zweite Möglichkeit ist die, dass das $u$ der entsprechenden Wörter im Ostseefinnischen sekundär ist. Leider sind beide Wörter in den fimnisch-ugrischen Sprachen nur auf einem recht engen Gebiet verbreitet, so dass keine Möglichkeit besteht, mit den Mitteln der vergleichenden Forschung festzustellen, ob das Finnische oder das Tscheremissische den älteren Zustand repräsentiert.

\section{WOu.}

Beispiele hierfür haben wir etwa 75 , von denen fast alle schon bei Beкe, Cs. Ny. 43-46, erwähnt sind. Einige seien noch hinzugefügt: kut'śà 'Eingeweidewurm', kuzôk 'Mitgift', šuar 'hölzerner Mörser', ukšând'žam 'sich erbrechen'. In den 
tschuwassischen Lehnwörtern ist das $u$ auffallend selten, es kommt nur in etwa 10 Fällen vor; vielleicht gab es im Tschuwassischen keine direkte Entsprechung des tscher. $u$.

Bereits im Urtscheremissischen erschien $u$. Auch dieser Vokal ist nicht einheitlichen Ursprungs, aber die Hauptentwicklungslinie ist doch leichter sichtbar als beim urtscher. o.

a) Die klare Mehrheit bilden die Fälle, in denen $u<$ vortscher. ${ }^{*}$. Diese Entwicklung sehen wir in den Wörterı juž, jù

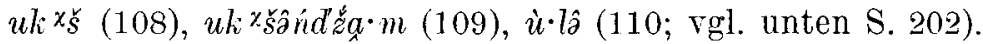

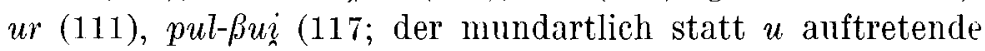
reduzierte Vokal ist deutlich sekundär und dürfte dadurch entstanden sein, dass pul-in diesem zusammengesetzten Wort in unbetonte Stellung gekommen ist), šur (119), š $u \delta e \cdot m$

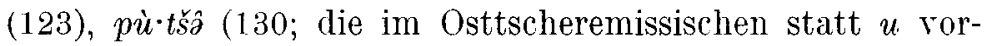
kommenden $\ddot{u}, i, \hat{\imath}$ sind sekundär, diese Formen können euphemistische Verdrehungen sein), šsk $x_{s}^{v}$ (138), muš (139). $p u \cdot s ̌ \hat{a}$ (143). Also 15 Beispiele.

$\mathrm{Zu}$ dieser Gruppe gehören aller Wahrscheinlichkeit nach noch folgende Wörter:

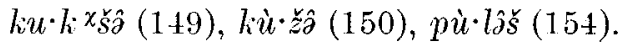

Vergleichen wir die Wörter, in denen $*_{0}>u$, mit denen. wo $*_{0} \equiv$ (s. oben), so bemerken wir, dass die Lage sehr ähnlich der beim ursprünglichen ${ }^{*} a$ ist; es lässt sich nämlich keinerlei Regel für dic Gründe der Zweiteilung aufzeigen. Man könnte vielleicht annehmen, dass ähnliche phonetische Faktoren, wie sie zu der sporadischen Entwicklung $\left({ }^{*} a>\right){ }^{*} a>0$ beigetragen haben dürften, auch die Entwicklung $*_{o}>u$ beeinflusst haben. So findet sich bei $5-6$ Wörtern in unmittelbarer Nachbarschaft des $u$ ein Labialkonsonant (anderseits haben wir auch drei Beispiele dafür, dass sich das o in solcher Stellung erhalten hat), bei $4-6$ Wörtern ein $k$ (ganz ebensoviel Beispiele gibt es aber dafür, dass sich o neben $k$ erhalten hat); besondere Beachtung verdienen die $-k$ š-Wör-

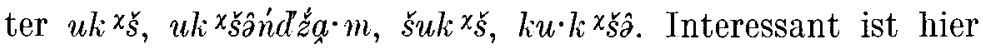
die Feststellung, dass $o$ und $u$ als Fortsetzer des ursprünglichen ${ }^{*} o$ von ziemlich gleicher Frequenz sind, während unter 
den Fortsetzern des $*_{a}$ das o gegenüber dem ${ }^{*} a$ deutlich zurücktritt.

b) Ihrer Zahl nach an zweiter Stelle stehen die Fïlle, in denen urtscher. $u<$ vortscher. * $u$ : šua $\%$ (170), lu 'zehn' (185), lu 'Knochen' (188), šu (204), ̀̀ (215). I)iese fünf Wörter sind alle solche. in denen der Vokal der ersten silbe infolge der lautlichen Entwicklung ans Ende des Stammes oder unmittelbar neben einen anderen Tokal greraten ist. Schon Stenitz hat den Charakter dieses sonderfalles festgestellt (Vok. 96), verallgemeinert aber vermutlich 'zu sehr, wenn er annimmt, dass in a 11 e $n$ Fällen, in denen wir im Auslant und ror Vokal statt des zu erwartenden reduzierten Vokaks einen Vollvokal sehen. die redurierten Vokale des Urtscheremissischen später mit den entsprechenden Vollyokalen zusammengefallen seien. In den ebenerwähnten fünf Wörtern hat es die sonst regrelmässige Reduktion des vortscher. ${ }^{*} u$ vielleicht niemals gegeben; im Frühurtscheremissischen scheint iln $u$ gedehnt gewesen zu sein (wie wir in einigen Dialekten noch heute im Auslaut $u$ bzw. uu antreffen), sei es nun infolge einer Verschmelzung des folgenden Konsonanten mit dem $u$ zu einem Vokal, der länger als ein kurzer Vokal war, oder infolge einer Ersatzdehnung, die beim Schwund des Konsonanten aufgetreten ist.

c) Drei Beispiele haben wir für die Entwicklung urtscher. $u<$ vortscher. * $\bar{u}$ : kul (234), pu (235), š $u e \cdot m$ (236). Die beiden letzteren Fälle unterscheiden sich, rom Standpunkt des Tscheremissischen selbst gesehen, in keiner Weise von den oben behandelten Fïllen, in denen urspr. ${ }^{*} u>$ urtscher. $u$ im Auslaut und vor Vokal. Mit anderen Worten, auf Grund eines im Urtscheremissischen herrschenden Lautgesetzes wäre man auch damn zu den Formen $p u, s ̌ u e \cdot m$ gekommen, wenn diese Wörter ursprünglich kein langes, sondern ein kurzes $u$ gehabt hätten.

d) Für die Entwicklung $u<$ vortscher. ${ }^{*} \bar{o}$ scheint es keine sicheren Beispiele zu geben. Wahrscheinlich gehört jedoch hierher $t \grave{u} \cdot m \hat{\partial} s ̌(168)$.

In unserer bisherigen Betrachtung haben wir gesehen, dass 
ursprüngliches ${ }^{*} \bar{u}$ und ${ }^{*} \bar{o}$ sich miteinander vermischt haben; beide sind Grundlage sowohl für urtscher. o als auch für urtscher. $u$ gewesen.

e) $u<$ vortscher. ${ }^{*} a$. Hierfür gibt es vier Beispiele: $n \grave{u} \cdot \delta \hat{a}$ (30), pù $\cdot \hat{a}$ (34), $\beta u \cdot r \delta \hat{a ̂ ~(55 ; ~ d a s ~ m u n d a r t l i c h ~ s o w o h l ~ i m ~ W e s t-~}$ wie im Osttscheremissischen anzutreffende $=$ ist offensichtlich

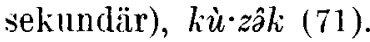

Diese Fälle sind sehr interessant und lehrreich. Sie zeigen, wie vortscher. ${ }^{*} a$ im 'Tscheremissischen manchmal einem mehrphasigen Prozess des Geschlossenerwerdens unterworfen worden ist: ${ }^{*} a>{ }^{*} \mathfrak{a}>{ }^{*} o>\boldsymbol{~}$. In den meisten Wörtern kam dieser Prozess auf der ${ }^{*} \hat{a}$-Stufe zum Stillstand, in anderen ging er weiter bis zum $o$ und in den jetzt zu betrachtenden Wörtern sogar bis zum $u$. Das Verhältnis $p \grave{u} \cdot r \hat{\partial} \sim O p \grave{o} \cdot r \hat{\partial}$, porema.m $\sim W$ parè $m a ̈ m, ~ O ~ p a r e m a \cdot m$, bei dem sich auch die ${ }^{*} \mathfrak{a}$-Stufe erhalten hat (vgl. oben S. 187), enthüllt deutlich, wie die Entwicklung abschnittweise vor sich gegangen ist. Aller Wahrscheinlichkeit nach waren ${ }^{*} a>0$ und ${ }^{*} o>u$ g l e i c h ze it ig e Veränderungen; daher kommt es auch, dass das aus * $a$ entstandene $o$ so selten an dem Lautwandel o) $>u$ teilgenommen hat.

Iöglicherweise ein Fall der Entwicklung ${ }^{*} a>u$ ist noch $k u \cdot r n \hat{z} z$ (13). Da dieses Wort onomatopoietischen Ursprungs ist, kann uns die lautliche Buntheit in seinen Entsprechungen in den anderen finnisch-ugrischen Sprachen in keiner Weise wundern. Das Finnische und das Lappische zeigen jedenfalls $a$-Vertretung. Wenn das Tscheremissische hinsichtlich der Vokalvertretung des betreffenden Wortes mit diesen Sprachen zusammenhinge (die permischen Sprachen mit ihrer $i$-Vertretung liegen auf einer anderen Linie), könnte man darauf hinweisen, dass bei dem in Rede stehenden Wort auf den Vokal der ersten Silbe $r$ folgt wie bei den Wörtern $p \grave{u} \cdot r \hat{o}$ und $\beta u \cdot r \delta \hat{\partial}$. Vielleicht also hat $r$ den Wandel $o>u$ besonders begünstigt; das lange $a$ in der finnischen Entsprechung kaarne wiederum dürfte auf der vokalverlängernden Wirkung beruhen, die mit $r$ beginnende Konsonantenverbindungen in den ostseefinnischen Sprachen oft gehabt haben.

Entweder auf $*_{a}$ oder auf $*_{o}$ geht das $u$ im Worte $t u m$, 
$\grave{u} \cdot m \hat{a}(45)$ zurück. Wenn nur die tscheremissischen und mordwinischen Wörter bekannt wären, wäre *toma die natürliche Rekonstruktion der Urform. Das Verhältnis derselben zum finnischen tammi dürfte sich kaum erklären lassen; es sei nur auf das bei den Benennungen von Bäumen gewöhnliche lautliche Schwanken hingewiesen.

$$
\text { 5. W (K) } o,(\mathrm{JV}) u \sim 0 u \text {. }
$$

Für dieses Lautverhältnis gibt es reichlich 15 Beispiele, rgl. Beкe Cs. Ny. 42. Die Mehrzahl derselben, etwa 10 Fälle, sind solche, in denen der Vokal am Ende des einsilbigen stammes steht oder in denen unmittelbar auf ihn ein anderer Vokal folgt. Fraglich ist es, ob die Wortstämme anderen Typs, in denen das gleiche Lautverhältnis herrscht, überhaupt eigentlich hierher gehören. Bei ihnen kann es sich um einen tranz zufälligen $o \sim u$-Wechsel handeln, ebenso wie anderseits in einigen Wörtern sporadisch im Westtscheremissischen $u$, aber im Osttscheremissischen $o$ anzutreffen ist.

Die Zahl der Wörter, die zu dieser Gruppe gehören, ist im yranzen so klein, dass es äusserst schwer fiele, in ihnen einen selbständigen urtscher. Vokal von der Art des $o$ anzunehmen, der sich im K-Dialekt zu $o$, sonst aber zu $u$ entwickelt hätte. Offenbar muss eine Alternative angenommen werden: entweder urtscher. $o$ in $\mathrm{K}$, sonst $>u$ oder urtscher. $u$ ausser im K-Dialekt. Für die erste Möglichkeit tritt SteINITz Vok. 99 ein, für die zweite Beke FUF XXIII 76-77. Die Auffassung von Steinitz lässt sich durch einen Hinweis auf die tscheremissischen Dialektverhältnisse stark stützen: In den Mundarten, wo nach seiner Meinung der Lautwandel $0>u$ stattgefunden hat, kommt $o$ wirklich niemals im Auslaut und vor Vokal vor. Mit anderen Worten wäre das $o$ in diesen Fällen mit dem in gleicher Stellung auftretenden $u$ verschmolzen, das oben in den Abschnitten b) und c) des t. Kapitels behandelt worden ist. Die beste Stütze für die Auffassung von Beke ist die geringe Verbreitung der $o$-Vertretung, wenn auch natürlich die Frage ungeklärt bliebe, 
warum das $u$ sich gerade in diesen Wörtern der K-Mundart zu o geöffnet hätte, obwohl es sich sonst auch hier in vielen Wörtern des gleichen Typs erhalten hat. Wahrscheinlich hat Steinitz Recht, aber selbst wenn es sich umgekehrt verhalten sollte, so handelt es sich hier $11 \mathrm{~m}$ eine Einzelheit, die nicht von wesentlicher Bedeutung für die Entwicklungsgeschichte des tscheremissischen Vokalsystems sein dürfte. Die Wörter. die dieses Lautverhältnis aufweisen und Entsprechungen in den verwandten Sprachen haben, sind nur wenige.

a) Ursprüngliches $* \bar{a}$ scheint an Hand der Vertretung im Ostseefinnischen der Vokal der ersten Silbe in $\xi_{0} \cdot a m, \check{s} u a \cdot m$ (37) zu vertreten, vgl. FUF XXX 6, 40. Da jedoch $\bar{a}$ in der fi.-ugr. Ursprache nicht vorgekommen sein dürfte, haben wir Grund, eine sporadische Vokallïngung anzunehmen ( ${ }^{*}$ sake- $>$ ${ }^{*}$ sāke- $>{ }^{*}$ säye- $>$ saa-). $\left(\mathrm{Ob}{ }^{*} \bar{a}\right.$ schon früher als im Frühurfinnischen aufgetreten ist, wissen wir nicht. Auch bei ko $\mathrm{em}$. $k u e \cdot m$ 'graben' (16) müssen wir von *a ausgehen (wenn nicht ron ${ }^{*}$, vgl. das Lappische!). falls die für dies Wort angenommene Etymologie richtig ist.

b) Ein $*_{0}$-Fall ist šoe, šue (124).

c) Ein *u-Fall: lo.e $m$, liue $\cdot m$ weben' (183).

d) Entweder von ${ }^{*} u$ oder von $*_{0}$ müssen wir bei mo mua $m(190)$ ausgehen.

\author{
6. $\mathrm{W}(\mathrm{K}) \hat{\partial},(\mathrm{JO} \mathrm{V}) \odot,(\mathrm{JP})=\sim \mathrm{O}$ (in den \\ meisten Dialekten) $u$, (JT CK () $\circ,($ J U CKr.) $\approx$.
}

Obwohl uns nicht über alle Dialekte genaue Angaben zur Verfügung stehen, dürfte die Lautvertretung - in erster Linie nach den Wortangaben in einigen Aufsätzen von BEKF. - in den Hauptzügen die obige sein.

Beispiele gibt es etwa 120; den von Beke, Cs. Ny. $46-50$. erwähnten ungefähr 70 Wörtern kann man u.a. hinzufügen julem (mundartlich im Osttscheremissischen $j u->j u ̈-) ~ ' b r e n-$ nen', jumâ 'Gott', jut ( O jüt) 'Nacht', kußâl 'Blase', kudalam 'laufen (vierfüssiges Tier)', kunâz 'Schmutz, Kehricht', kupem 'schimmelı', kupšuto 'Holzhäher', kuptâryem 'sich fal- 
ten, runzeln', kurôś 'grosse Tasche aus Birkenrinde', kutkâ 'Ameise', luk 'Innenecke', lumem 'durch Hexerei verblen-

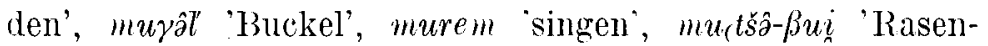

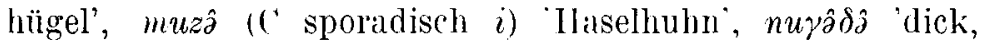
dicht', pundž́em 'drehen, auspressen', puryedam 'wühlen', purj’âž ( W mundartlich u) 'Schneegestöber', puškedam 'Durchfall haben', putrali 'sehr', pu,tšosstem 'prickelln, kneifen', pužar 'Hobel', rudem 'loslassen', rumbâli 'trübe', šumem 'schleifen', tungâr 'äusserste Rinde', tupka 'gekämmte Wolle', umbal 'jene Seite', upša, umša 'Mund, urem 'mit Erde bedecken', urža 'Roggen', uškal 'Kuh'. - 'Tschuwassische Lehnwörter, die die gleiche interdialektale Vertretung aufweisen, gibt es ziemlich reichlich, wenigstens um 20.

Im Urtscheremissischen hat es in dieser stellung ein reduziertes $\approx$ gegeben (vgl. STrisiTz Vok.96), das sich entweder unverändert oder als etwas offenerer Laut $(\Leftrightarrow)$ in den Mitteldialekten erhalten hat, während es in der K-Mundart delabialisiert worden und in dem weiten osttscheremissischen Gebiet zum Vollvokal $u$ geworden ist. Über den phonetischen Charakter der reduzierten Labialvokale s. FUF XXIX 226, 248 .

Die Entwicklungsgeschichte des urtscher. $\approx$ ist ausserrewöhnlich einheitlich.

a) In der erdrückenden Mehrheit der Fälle vertritt ${ }^{*} \approx$ vortscher. * $u$. Vgl. Gosmor" NyK XXXIX 274 , RAviLa FUF XX 107, STEINIT'z Vok. 22. Beispiele sind: $j \hat{\partial} \cdot m \hat{\partial}, j \hat{u} \cdot m \hat{\partial}(173), k \hat{\partial}-$,

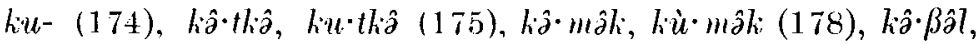

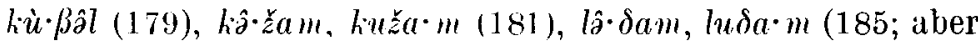
urtscher. ${ }^{*} u$ im Auslaut: W O lu 'zehn', vgl. oben S. 197), lâm,

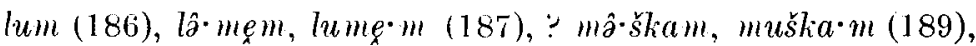

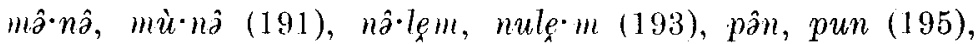

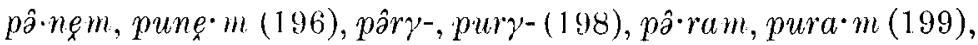

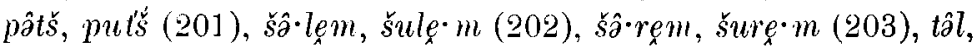

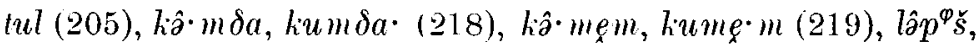

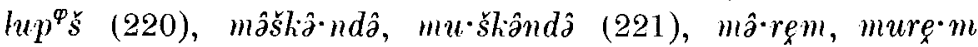

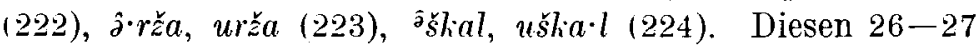
Wörtern kann man als offensichtliche ${ }^{*} u$-Wörter noch folgende hinzufügen, die in den permischen Sprachen genau zu 
der Vertretung im 'Tscheremissischen passende Entsprechungen haben:

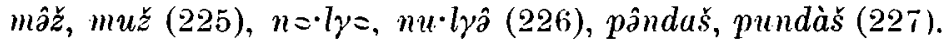
pâ·ręm, purę $m$ (228), pâš, puš (229), W (K) tâ·litâ (230).

Somit beträgt die Zahl der Beispiele für die Entwicklung vortscher. ${ }^{*} u>$ urtscher. $\approx$ über 30 .

b) Fälle, in denen urtscher. $\approx$ sich sporadisch aus $*_{\prime \prime}$

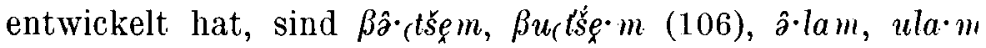
(110; aber urtscher. $u$ : W O $\dot{u} \cdot l \hat{a}), \beta^{\mathfrak{\partial} z} a \cdot l e m, u \stackrel{x}{z} a l \underline{\alpha} \cdot m$ (112). In diesen Wörtern ist wahrscheinlich ${ }^{*} o>$ urtscher. ${ }^{*} u$, das dann unregelmässig reduziert worden ist. Zwei von ihnen beginnen mit $\beta$. Sollte das urtscher. $u$ in der Verbindung $\beta u-$ die Neigung gehabt haben, sich $\mathrm{zu} \approx \mathrm{zu}$ entwickeln? Das ist glaubhaft, denn gemeintscheremissische, eigene Wörter. die mit $\beta u$ - anfangen und in allen Mundarten den Vollvokal aufweisen, dürfte es ausser $\beta u$ (107) keine geben. Bei de॥ übrigen mit $\beta u$ - beginnenden Wörtern, nämlich $\beta u l n a ̂$ 'Zinn'. $\beta u r \delta \delta$ ( $\check{5}$ ) und $\beta$ urt 'Litze', kommt mundartlich auch $s-V e r-$ tretung vor. Zweitens gehört $\beta^{\tilde{\partial} \check{z} a} \cdot l e m, u \tilde{z} a l_{\alpha} \cdot m$ zu einem Worttyp, bei dem die erste Silbe ständig unbetont ist. Dass Unbetontheit Rednzierung des $u$ veranlasst hat, beweist u.a.

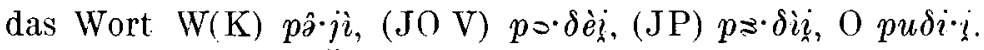

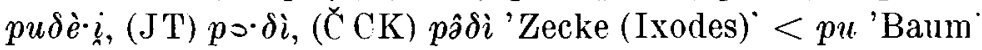
$+t i i$ 'Laus'. Bei dem Verb $\hat{\partial} \cdot l a m, u l a \cdot m$ ist die Reduzierungr des $u$ offenbar durch das stetige Vorkommen dieses Wortes in der alltäglichen Rede veranlasst worden. Der alte Verbalstamm $\grave{u} \cdot l \hat{\jmath}$ hat vielleicht sogar aus zwei Gründen ein Sonderstellung eingenommen: Erstens hat seine für eine Präsensform der 3. Person Sing. einzigartige Struktur (vgl. deı analogischen Typ $\hat{\partial} \cdot l e \breve{s}$ im Westtscheremissischen) das $u$ del ersten Silbe betont erhalten, zweitens steht es seiner Bedeutung nach teilweise ausserhalb der übrigen Konjugationsformen, da es auch als Nomen in der Bedeutung 'was vorhandeı ist, all, ganz' gebraucht wird.

Von $*_{o}$ muss man vielleicht auch in dem Zahlwort kâm. kum (95) ausgehen. Zwar scheint das entsprechende lappische Kardinalzahlwort gol'bmâ (nicht aber das Ordinalzahlwort goalmad) ebenso wie das Tscheremissische anf ein 
ursprüngliches $u$ hinzudenten. Auch rer Vokal der ersten Silbe im entsprechenden mordwinischen kolmo kann ebensogut auf $u$ wie auf $o$ zurückgehen. $O b$ nun die lappische und tscheremissische Vertretung Ergebnis einer in beiden Sprachen getrennt vor sich gegangenen unregelmässigen Entwicklung ist oder sich aus einer älteren Doppelheit $o \sim u$ in diesem Zahlwort erklärt, in jedem Fall widerspiegelt sie das den Zahlwörtern eigene lautliche Schwanken.

c) Auf ursprüngliches $*_{i}$ geht urtscher. $\approx$ in den Wörtern šâ· $m e m, \breve{s} u m e \cdot m(364)$ und $\bar{a} z a r, u z ̌ a \cdot r(392)$ zurück. Hier hat der Hintervokal, der in der zweiten silbe gewesen ist ${ }^{*}$ šina-. *viša-), den vorhergehenden Vokal sporadisch velarisiert. Wahrscheinlich hat auch in dem Wort šažar; šužar, šüũar (389) im Urtscher. ₹ gestanden. Wodurch die rerhältnismässig wenig verbreitete Variante mit dem Vordervoka] (n) entstanden ist, bleibt dunkel. Wenn das Wort ein baltisches Lehnwort ist, die dem finn. sisar direkt an dic Seite zu stellen ist, handelt es sich hier um die Entwicklung $* i>\dot{z}$. die aus dem gleichen Grunde stattgefunden hätte wie in den Wörtern šâ.mem und âžar. Man muss jedoch auf Grund des entsprechenden estn. sõsar auch die Entwicklung $*_{e}>=$ für möglich halten. Wenn aber tscher. šâžar, šužar ein iranisches Lehnwort ist wie das entsprechende mordwinische und wahrscheinlich auch das entsprechende permische Wort, so ist die Entstehung des $\approx$ der ersten Silbe aus dem danu anzunehmenden ${ }^{*} a$ cine ganz anssergewöhnliche Erscheinung.

$$
\text { . } \quad \text {. W } \ddot{a} \sim(1 a \text {. }
$$

Beispiele hierfür gibt es etwa 50 , davon bei BekE Cs. Xy. 31-33 ungefähr 30. Ausser diesen seien angeführt: apšat 'Schmied', aršaš 'Perlenband', jarnem 'schwach, entkräftet werden', kaßarem 'sterben', kakainlem 'gackern (Henne)', kala 'wohl', ka t'š̀a 'Bursche', lańdż̀ara 'schwach, liraftlos', lazra id., maska 'Bär' (in $\mathrm{K}$ ö), nańa 'Brot' (Kinderwort), papem 'schlafen' (Kinderwort), partš́a 'Ähre', šallaka 'Knöchel'. šarnem 'sich erinnern', tazâla 'Schleim'. Gemeintscheremis- 
sische Lehnwörter aus dem Tschuwassischen mit $\mathbb{W} \ddot{a} \sim 0 a$ in der ersten silbe gribt es in qrosser Menge, wenigstens 80. Räsäxex, T'schuw. Lehnw. 74, nimmt all, dass das Urtscheremissische hier ${ }^{*} a$ gehat hot hat, das im Westtscheremissischen zu ä geworden ist. Die Auffassung von Wichmasx, FUF XVI Anz. $44-45$, ist genau entgegengesetzt: urtscher. ${ }^{*} \ddot{a}>0$ ( $a$. Er bestreitet die Bedeutung der drei Etỵmologien, mit deren Hilfe Räsänen zeigen will, dass das Lautverhältnis $\mathrm{W} \ddot{a} \sim 0 \mathrm{O}$ in den anderen fi.-ugr. Sprachen hintervokalische Entsprechungen habe. Aber man kann den sieben Etymologien, die nach Wichmanns Meinung den vordervokalischen Ursprung dieses Lautverhältnisses beweisen, wohl kaum mehr Gewicht beimessen. Drei von ihnen, nämlich die der Wörter W liär, () kar, W lä·nzarä, O landž̉ara und W nä·läm, () nala $m$, sind ohne weiteres zu verwerfen. Auf zwei Arten

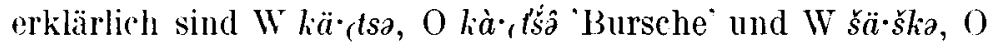
ša.šk ? 'Otter'. Mit ersterem vergleicht Wichmann wog. käs' 'jüngerer Bruder, Neffe' ('Torvosex daregen wog. $\chi \bar{u} s$ 'I)iener', s. FCF XIX 1+3, XXXI 131), mit letzterem weps. hähl 'mustela lutreola'. Diese Nebeneinanderstellungen sind deshalb unsicher, weil im Tschuwassischen die Wörter kattśŝ 'Bursche, Bräutigam' und šašhâ 'Nörz' ( Räsänen, a.a.O. 245, 264) vorkommen, und wenigstens das letztgenannte für eine Entlehmung aus dem Tscheremissischen zu halten, fällt etwas schwer, denn es tritt auch im Tatarischen, Baschkirischen und

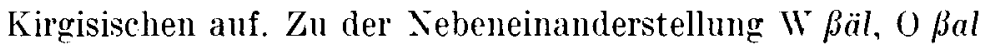
'Oberfläche' fi. pääli 'id'. muss man bemerkten, dass es nicht sicher ist, ob fi. pääli-, päälli- ( päälle 'auf, über c. Acc.', päällä 'oberhalb, über, auf c. Dat.', pä̈̈ltä 'von oben, von aussen') überhaupt ein von Anfang an selbständiges Wort ist. Die dazugehörigen, in Klammern stehenden Postpositionen verbindet das Sprachgefühl mit pää 'Kopf, Ende' und insbesondere die Analyse der Form päälle ist unmöglich, wenn man von einem ursprünglichen Stamm pääli- ausgeht; hingegen wäre sie ein ganz regelmässiger Allativ des Wortes pää. Es ist also sehr möglich, dass pääli-, päälli- eine Abstraktion ist, die auf den äusseren Lokalkasus des Wortes pää und der Ableitung päällinen 'Überzug, Bezug; oberhalb be- 
findlich' beruht. Aus lautlichen Gründen unsicher ist die Zusammenstellung von tscher W $k \cdot e \cdot j e x m(<* k a ̈ \cdot j e m), 0$ lia-

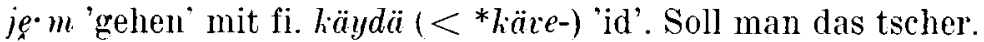
$j$ als Bindekonsonant oder als Ergebnis einer Delabialisierung $*_{v}>j$ auffassen? Wenn man in Betracht zieht, dass es auch im 'Tschuwassischen ein Verb kaj- '(weg)gehen, fahren' (Räsänen, a.a.0. 244) gibt, so ist es möglich, dass tscher. kajem $m$ aus dieser sprache stammt.

Das einzige Wort rom Typ W $\ddot{a} \sim() a$, das sicher finnisch-

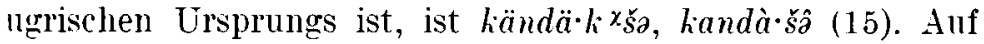
Grund der etymologischen Entsprechungen dieses Wortes würde man in ihm Hintervokale erwarten, aber die Palatalität seines Vokalismus erklärt sich leicht als Einwirkung des

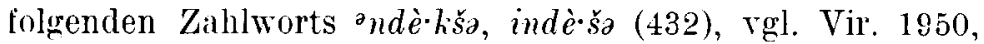
s. $70-71$.

Es sind jedoch noch folgende Würter zu erwähnen:

'T'scherW (K) kärə̌̆, kärš 'russische Gusli', wotj. kịreź, lived'ź 'Gusli; Geige ' mordM kard'zi 'Geige, Violine'. Hier' müsste man auf Grund des Mordwinischen von * $a$ ausgehen (im Wotjakischen hätte man in jedem Fall eine unregelmässige Entwicklung anzunehmen, weil der Vokal der ersten Silbe in liveź der Vertretung im Mordwinischen und Tscheremissischen nicht entspricht). Im Hinblick auf die Bedeutung des in Rede stehenden Wortes erscheint es in diesem Fall fast als natürlicher, Lehnbeziehungen zu vermuten als eine Urrerwandtschaft vorauszusetzen; die Erforschung der Gegenstände könnte rielleicht Licht in die Frage bringen, ob schon in der wolgafinnisch-permischen Zeit ein Instrument von ler Art der Gusli oder der Geige bekannt sein konnte.

TscherW (K) šärtñ, O šartñ̂, (C Č) sertrie., (JT) šerrtne, ( LJ) šör 'riö 'eine Weidenart' fi. saarni, liv. särna 'Esche'. Wegen der zweifachen Vertretung $a \sim e(>\mathrm{CJ} \ddot{o})$ im Osttscheremissischen kann man nicht entscheiden, ob der Vokal der ersten Silbe ursprünglich zu dem jetzt in Rede stehenden oder zu dem später im 8 . Kapitel zu behandelnden Typ gehört. Ebenso dunkel bleibt es, ob das Wort im Vortscheremissischen vorder- oder hinterrokalisch gewesen ist; die livische Entsprechung mit illrem $\bar{a}$ passt mit dem tschere- 
missischen Wort unbedingi besser zusammen als die finnische mit ihrem $\bar{u}$. Im Ostseefinnischen ist anscheinend eine Längung des Vokals vor mit $r$ beginnenden Konsonantenverbindungen eingetreten.

Die verwandten Sprachen verhelfen uns offenbar zu keinel Klarheit über den Ursprung des Lautverhältnisses $W \ddot{a} \sim \mathrm{O} a$ : Zuverlässiges etymologisches Vergleichsmaterial fehlt völlig. $\mathrm{W} \ddot{a} \sim \mathrm{O} a$ erweist sich als eine Parallelerscheinung zu dem im 1. Kapitel behandelten urtscher. ${ }^{*} a$; sein Auftreten beschränkt sich, abgesehen von Lehnwörtern, fast ausschliesslich auf den rein tscheremissischen Spezialwortschatz, an dem die deskriptiven und onomatopoietischen Wörter einen bedeutenden Anteil haben. Nur gelegentlich hat sich ein älteres Wort diesem Typ angepasst. Zweifellos muss $\mathrm{W} \ddot{a} \sim \mathrm{O}$ a dem urtscher. ${ }^{*} a$ an die Seite gestellt werden, sowohl chronologisch wie auch als Seitenstück desselben im phonologischen System des späturtscheremissischen Vokalismus. Mit anderen Worten: Im Urtscheremissischen ist das erwähnte Lautver-

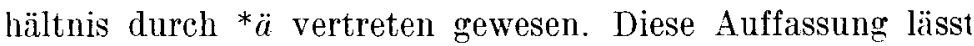
sich sehr gut auch damit begründen, dass die Entwicklung des urtscher. ${ }^{*} \ddot{a}$ in anderen als der ersten Silbe die gleiche gewesen ist: Seine Vertretung ist $\mathrm{W} \ddot{a} \sim \mathrm{O} a, \mathrm{z} . \mathrm{B}$. W ne.läm, O nela $\cdot m$ 'schlucken, verschlingen'.

Die Frage, wie *ä in den Vokalismus der ersten Silbe inı Späturtscheremissischen gekommen ist, lässt sich im wesentlichen mit der gleichen Erklärung beantworten, die auch für die Entstehungsgeschichte des $a$ der ersten Silbe gilt. Ebensı wie vortscher. ${ }^{*} a$ verschwand im Frühurtscheremissischen auch vortscher. ${ }^{*} \ddot{a}$ aus der ersten Silbe infolge verschiedenel' Lautveränderungen. In anderen als der ersten Silbe blieb ebenso wie ${ }^{*} a$ auch $* \ddot{a}$ erhalten (allerdings ging dieses nicht auf das vortscher. ${ }^{*} \ddot{a}$ zurück, sondern war eine durch die im Urtscheremissischen herrschende Vokalharmonie hervorgerufene vordervokalische Variante von a). Aus diesen zahlreichen Stellungen - man denke nur an die Kasus -lä, -län. die Personalendungen $-\ddot{a} m,-\ddot{a} t,-n \ddot{a},-\delta \ddot{a}$, von denen die bejden letzten zugleich Possessivsuffixe sind, die Endungen der

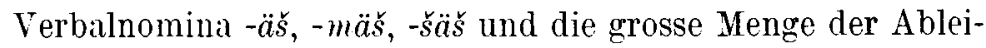


tungssuffixe mit $\ddot{a}-$ hatte das $\ddot{a}$ gute Möglichkeiten, sich auszubreiten und auch wieder in die erste Silbe einzudringen. Vielleicht hat der Einfluss fremder Phonetik zu dieser Entwicklung beigetragen. Die vielen tschuwassischen Lehnwörter des Späturtscheremissischen, die man mit Wichmann als vor dem tschuwassischen Lautwandel $* \ddot{a}>a$ übernominen betrachten muss, haben die Stellung des * $\ddot{a}$ im Vokalismus der ersten Silbe wirksam festigen können. Mittelbar erstreckte sich ihre Wirkung vielleicht auch auf das a del ersten Silbe. Wenn nämlich $* \ddot{a}$, unterstützt vom fremden Einfluss, sich in dieser Silbenstellung festsetzen konnte. begann das in der Sprache herrschende Streben nach Harmonie und Gleichgewicht für $a$, den phonologischen Gegensatz $\mathrm{zu} * \ddot{a}, \mathrm{zu}$ arbeiten und auch $a$ errang nun eine beträchtliche Frequenz in dem Wortschatz, der im späteren Stadium des Urtscheremissischen geschaffen wurde. Interessant ist die Feststellung, dass urtscher. $a$ in der ersten Silbe auch im eigenen Wortgut der Sprache doch nicht so häufig auftritt wie $* \ddot{a}$, was gerade darauf hindeuten kann, dass es in diese Silbenstellung gewissormassen infolge des * $\ddot{a}$ und als Begleiter desselben gekommen ist.

\section{W (K) $\ddot{a},(\mathrm{JV}) e \sim 0 e$.}

Hierfür haben wir etwa 30 Beispiele. Einige Zusätze zu den von BЕке Cs. Ny. 13 aufgezählten seien noch genannt: Ber 'Stelle, Platz', ßereštam 'geraten, ergreifen', orthe 'Schachtel aus Birkenrinde' (auch der JO-Dialekt hat in diesem Wort

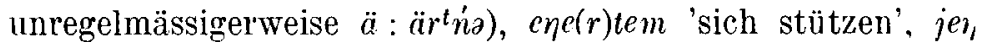
'Mensch, Seele' (im Osttscheremissischen anscheinend durch

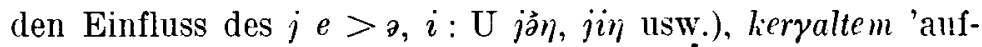
wickeln', leneš 'Eimer aus einem Holzstüc $\dot{k}$, mek $x_{s}$ 'Morschheit (des Baumes)', šenam 'durchwühlen', šer $a s ̌$ 'Ring' (iu vielen osttscheremissischen Mundarten durch den Einfluss

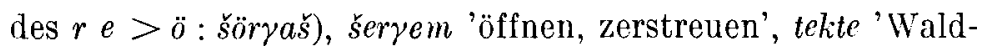
bienenschwarm'.

Beke äussert Cs. Ny. 13-14 die Ansicht, dass die ursprüng- 
liche Vertretung sich im K-Dialekt erhalten hat; auch die Vertretung in den verwandten Sprachen weist hier auf * $\ddot{a}$ hin. später, FUF XXIII 72-73, nahm er einen andern Standpunkt ein: Danach ist das osttscheremissische und in den westtscheremissischen $J$ - und $V$-Dialekten auftretende $e$ ursprünglich. Nach Steixitz hat sich in $\mathbb{W} \ddot{a}$ nicht nur die urtscheremissische, sondern geradezu die finnisch-ugrische Vertretung erhalten; als eigentliche Fortsetzung des fi.-ugr. * $\ddot{a}$ betrachtet er gerade das Lautrerhältnis $\mathbb{W}_{\ddot{a}} \sim \mathrm{O} e$ (Vok. $97,99)$. Es ist auch offensichtlich, dass man die westtscheremissische $\ddot{a}$-Vertretung, wenn sie sich auch auf die $\mathrm{K}$ IIundart beschränkt, nicht als Ergebnis eines späten Lautwandels $e>\ddot{a}$ ansehen kann. W $\ddot{a} \sim \mathrm{O} e$ vertritt eine besondere urtscheremissische Vokalkategorie, die allderdings eine recht geringe Frequenz gehabt hat. Wenn wir aber wie steinitz von urtscher. ${ }^{*} \ddot{a}$ ausgehen würden, müssten wir rom Standpunkt des 'Tscheremissischen zunächst fragen: Warum ist hier nicht $* \ddot{a}>\mathrm{J} \mathrm{V} \ddot{a}, \mathrm{O} a$ wie in den oben im 7. Kapitel behandelten Fällen? Auch etymologische Tatsachen sprechen dafür, dass $W \ddot{a} \sim 0$ e eine vom urtscher. ${ }^{*} \ddot{a}$ ganz unabhängige Vokalkategorie ist. Während nur ein oder zwei * $\ddot{a}$-Wörter etymologische Entsprechungen in den verwandten Sprachen haben, finden sich solche für reichlich 10 der zur Gruppe W $\ddot{a} \sim O e$ gehörenden Wörter, obwohl die Gesamtzahl der Wörter dieses Typs wesentlich kleiner ist als die der * $\ddot{a}$-Wörter. Wir sehen also, dass $W \ddot{a} \sim 0$ e auf einen Laut zurückgeht, der im urtscheremissischen Vokalismus vor der Herausbildung des sekundären *ä bekannt gewesen ist und in der urtscheremissischen Zeit mit diesem qualitativ nicht identisch gewesen sein kann. Die ganz natürliche Schlussfolgerung ist die, dass wir es hier mit der vordervokalischen phonologischen Entsprechung des urtscher. ${ }^{*} a$ zu tun haben, d.h. mit ${ }^{*} \varepsilon$, dem Zwischenvokal von $\ddot{a}$ und $e$, das sich parallel zu dem Lautwandel ${ }^{*} a \dot{a}>a$ in $\mathrm{K}$ zu $\ddot{a}$ entwickelt hat, während die östlicheren Mundarten des Westtscheremissischen, $\mathrm{J}$ und $\mathrm{V}$, sich diesmal der osttscheremissischen Gruppe angeschlossen haben, für die $* \varepsilon>e$ völlig parallel $\mathrm{zu} * \dot{a}>0$ ist. 
Über den Ursprung des urtscher. ${ }^{*} \varepsilon$ lässt sich Folgendes feststellen:

a) Vertreter des ursprünglichen $* \ddot{a}$ ist es in den Wörtern

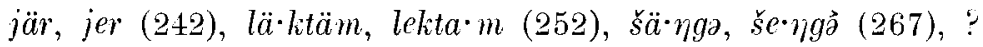

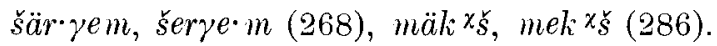

Erwähnt werden müssen auch einige Wörter, die im Tscheremissischen und in den permischen Sprachen, aber nicht weiter westlich vorkommen und in deren erster Silbe wir wahrscheinlich von $*_{\ddot{a}}$ auszugehen haben: $\beta \ddot{a} \cdot r \gamma \partial, \beta \epsilon \cdot r \gamma \grave{\jmath}$ (296),

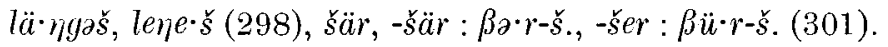

Die Vokalvertretung aller permischen Entsprechungen dieser Wörter, über die auf SS. 265, 305, 314 näher gesprochen wird, ist nicht derart, dass sie unbedingt die Ursprünglichkeit des * $\ddot{a}$ beweisen würde; dennoch erklärt sie sich am besten, wenn man von $* \ddot{a}$ ausgeht. Hinsichtlich tscher. šär, šer ist ausserdem festzustellen, dass die obugrischen Entsprechungen dieses Wortes deutlich auf * $\ddot{a}$ hinzuweisen scheinen: ostjV lèr . Kaz. lęrr usw. (urostj. *e), wog TČ $t^{e} \bar{a} r$, So. tär usw. (urwog. ${ }^{*} \bar{a}$ ). vgl. Stersitz Vok. 40.

Somit ergeben sich $4-8$ Beispiele für die Entwicklung vortscher. ${ }^{*} \ddot{a}>$ urtscher. ${ }^{*} \varepsilon$.

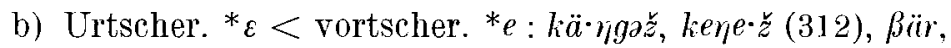

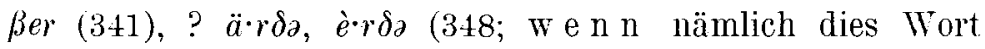
zu $\ddot{o} \cdot r \delta \partial \check{z}$ gehört). Insgesamt haben wir hier 2-3 Beispiele.

c) Entweder auf ${ }^{*} \ddot{a}$ oder auf ${ }^{*} e$ geht urtscher. ${ }^{*} \varepsilon$ zurück in den Pronomina $m \ddot{a}$, me (316) und tä, te (337). Es ist möglich. dass bei den Personalpronomina von altersher solche Vokalrariationen aufgetreten sind, wie sie z.B. im Finnischen noch heute bei den entsprechenden Possessivsuffixen und Personalendungen vorkommen: 1 . Pers. Pl. $-m m e \sim-m m a,-m m a ̈$, 2. Pers. Pl. -tte $\sim-t t a,-t t a ̈ ; ~-n n e \sim-n n a,-n n \ddot{a}$. Da die tscheremissischen Possessivsuffixe und Personalendungen - (W)

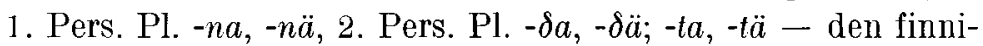
schen Suffixvarianten mit $a \sim \ddot{a}$ zu entsprechen scheinen, erscheint es nicht unmöglich, dass das offene ${ }^{*} \ddot{a}$ auch in den Personalpronomen vortscheremissischen Ursprungs ist. Eine andere Alternative ist die, dass das frühurtscher. ${ }^{*} \ddot{a}$ der Personalpronomina (> lautgesetzlich späturtscher. ${ }^{*} \varepsilon$ in der 
ersten Silbe) auf der Analogie der Personalendungen und Possessivsuffixe beruht.

So kärglich auch unser etymologisches Vergleichsmaterial ist, so gibt es doch ein recht deutliches Bild davon, dass urtscher. ${ }^{*} \varepsilon$ eigentlich nur vortscher. ${ }^{*} \ddot{a}$ vertritt. Das ursprüngliche $* \ddot{a}$ hat sich also in diesen Fällen nicht weit von seiner vortscheremissischen Qualität entfernt, wenngleich es etwas geschlossener geworden ist (vgl. die Entwicklung $\left.{ }^{*} a>{ }^{*} a\right)$. Wie wir später sehen werden, ist ${ }^{*} \varepsilon$ durchaus uicht der einzige Laut, der vortscher. ${ }^{*} \ddot{a}$ vertritt. Wir können leicht bemerken, dass das Auftreten von ${ }^{*} \varepsilon$ von der Qualität des folgenden Konsonanten abhängt: in 13 Fällen folgt auf den Vokal $r$, in 9 Fällen $\eta$ und in 4 Fällen eine mit $k$ beginnende Konsonantenverbindung. Diese Konsonanten haben also eine besondere Neigung gehabt, die ursprüngliche Qualität des in Rede stehenden offenen Vordervokals aufrechtzuerhalten. Dazu ist noch zu berücksichtigen, dass in den Pronomina $m \ddot{a}, m e ; t \ddot{a}$, te das ${ }^{*} \varepsilon$ die Möglichkeit gehabt hat, sich zu erhalten, weil im Auslaut ein Einfluss der Konsonanten nicht in Frage kam. Damn bleiben nur ein paar Fälle übrig, in denen die konsonantische Nachbarschaft des ${ }^{*} \varepsilon$ eine andere ist als die ebenerwähnte: W

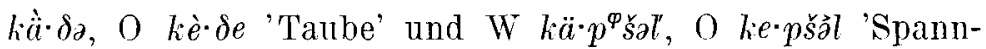
strick'; das erstgenannte (ein onomatopoietisches Wort:) ist das einzige Beispiel für ein Auftreten von ${ }^{*} \varepsilon$ vor einem anderen Dentalkonsonanten als $r$.

Die seltenen Fälle, in denen urtscher. ${ }^{*} \varepsilon<$ vortscher. ${ }^{*} e$, erklären sich als ebensolche sporadischen Schwankungen wie die $* \grave{a}$-Wörter, die auf eine Urform mit $*_{o}$ zurückgehen (s. oben S. 190). Sehr erläuternd ist $\beta a ̈ r, \beta e r \sim$ fi. vero. Auch im K-Dialekt finden wir ein $e$ in der Postposition Berts(an) 'wegen, anstatt', die der sog. 2. Elativ von $\beta \ddot{a r}, \beta c r$ ist. Da der seltene 2. Elativ nicht zu den gewöhnlichen Kasusendungren gehört, hat sich auch $\beta$ erts(an) offenbar schon früh vom eigentlichen Paradigma abgespalten und somit sein urtscher. $e$ beibehalten können, während im Stammwort selbst der sporadische Lautwandel ${ }^{*} e>{ }^{*} \varepsilon$ stattgefunden hat. Diesen Wandel hat natürlich das auf den Vokal folgende $r$ veranlasst, 
dessen in diese Richtung gehende Wirkung aus verschiedenen Sprachen bekannt ist. Wenn $\ddot{o} \cdot r \delta a z$ 'Seite' (348) wirklich von $\ddot{a} \cdot r \delta \partial, \grave{e} \cdot r \delta \partial$ abgeleitet ist, haben wir hier einen genau entsprechenden Fall. $\ddot{o} \cdot r \delta \partial \check{z}$ ist deutlich $<{ }^{*} e \cdot r \delta a z$ (von diesem Wort und seinem $\ddot{o}$ wird später auf $\mathrm{S}$. 214 die Rede sein), wobei in dem eventuellen Stammwort eine sporadische Entwicklung ${ }^{*} e \cdot r \delta \partial>{ }^{*} \varepsilon \cdot r \delta \partial$ vor sich gegangen wäre.

\section{W O e.}

Hierfür gibt es etwa 50 Beispiele, von denen sich bei Bнке Cs. Ny. 14-15 ungefähr 30 finden. Einige der übrigen Beispiele: Be•r, t'š̉n 'wegen, anstatt' (vgl. oben), kelam 'waten',

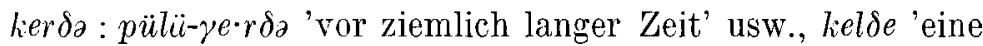
Art Netz', lep 'Milz', mer t'sem 'kränklich sein', nerem 'duseln', netšsk 'verwöhnt (Kind)', šer 'Perlenschmuck', temdem 'zudrücken, packen', teße 'sieh!'. Unter den tschuwassischen Lehnwörtern gibt as etwa 10 Beispiele für WO $e$.

Das $e$, das in allen Mundarten vorkommt, ist natürlich urtscheremissisch. Über seinen Ursprung lassen sich folgende Beobachtungen machen:

a) Auf ursprüngliches $* \ddot{a}$ geht es in 10-11 Fällen zurück: $j \grave{e} \cdot \check{z} \partial \eta, j \grave{\partial} \cdot \check{\partial} \eta \eta(243 ;$ in den anderen Dialekten ausser $\mathrm{K}$ anscheinend durch den Einfluss des $j e>2$ ), kela.m (245), $k e \cdot r \gamma \grave{\partial}(247), k e r t a \cdot m(248)$, lè $\cdot l \grave{\partial}$ (254), šere $\cdot \eta g \grave{a}$ (269; Belege für dieses Wort aus der K-Mundart stehen uns nicht zur Verfügung, so dass es auch zur ${ }^{*} \varepsilon$-Gruppe gehören $\mathrm{k}$ a $\mathrm{n} n$ ), $t \grave{e} \cdot l \grave{\partial}$ (271), pè $\cdot l d$ (282), mel (285), elte (290; im Osttscheremissischen mundartlich sekundärer Lautwandel $e>\ddot{o})$, kem (292).

Der Konsonant, der auf den Vokal der ersten Silbe folgt, ist in sechs Fällen $l$, in drei Fällen $r$, in einem $z$ und ebenfalls in einem $m$. Schon Steinitz hat mit gutem Grund erklärt, dass fi.-ugr. ${ }^{*} \ddot{a}$ lautgesetzlich vor $l>e$ (Vok. 99). Merkwürdig ist das Auftreten des gleichen Lautwandels vor $r$, wo man ${ }^{*} \varepsilon$ erwarten müsste, vgl. oben S. 210. Die $e$-Vertretung dürfte als Reihenübergang anzusehen sein; waren $\operatorname{doch}{ }^{*} \varepsilon$ und $e$ einander qualitativ recht nah. 
b) $e<$ vortscher. ${ }^{*} \bar{e}$ in vier Fällen: lem (35̄5), nela $\cdot m \cdot(3 \bar{\jmath})$. pel (358) und ner (361).

c) $3-4$ Beispiele gibt es für die Entwicklung $e<$ vortscher.

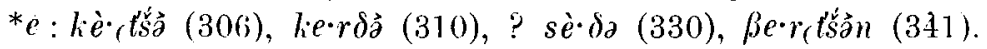

Selbst wenn wir auch die oben auf S\$. 210-211 erwähnten Fälle berücksichtigen $\left({ }^{*} \beta \varepsilon r\right.$, ? $\left.{ }^{*} \varepsilon \cdot r \delta z\right)$, hei denen der Vokal der ersten Silbe sich dadurch erklärt, dass man eine Entwicklung ${ }_{\varepsilon}$ $<$ frühurtscher. ${ }^{*} e$ annimmt, können wir das urtscher.e nicht als normale Vertretung des vorticher. *e betrachten. Ebensoweniy können wir das in einigen anderen, später zu besprechenden Fällen, in denen dem vortscher. *e im Tscheremissischen irgendein Vollvokal entspricht. Meistens nämlich ist das $*_{e}$ hier reduziert worden (einen Sonderfall bilden die stämme. in denen der Vokal im Auslaut oder Anfangskomponente eines auf $i$ endenden Diphthonges ist oder unmittelbar vor cinem anderen Vokal steht; in ihnen ist ein reduzierter Vokil nicht mögrlich). Von Bedeutung kann der Umstand sein, dass in Wörtern, die einen Vollvokal enthalten, (ausgenommen dip ebenerwïhnten Sonderfälle) in der zweiten Silbe im Vortscheremissischen regelmässig * $\ddot{a}(\mathrm{~b} z w . * a)$ gestanden hat. Das rilt offenbar auch für das Wort $\beta \ddot{r}$, $\beta e r$, das, obwohl es apokopiert ist, auf die Form *verä zurückzugehen scheint. vgl. Vir. 1948, S. 139. Es gibt ja auch ziemlich viele andere Ausnahmen von der Regel, dass die Vertretumg des ursprünglichen $* a, * \ddot{a}$ der zweiten Silbe im Tscheremissischen von der Apokope verschont geblieben ist, vgi. FUF XXIX 232. Ferner besteht die Möglichkeit, dass für das ${ }^{*} e$ der ersten Silbe schon früh kombinatorische Varianten aufgetreten sind, je nach dem Vokal der zweiten Silbe. Die Vertretung in den permischen Sprachen (und vielleicht im Lappischen) deutet darauf hin; vor $*_{e}$ könnte die Artikulation des in Rede stehenden Vokales um einiges gespannter gewesen sein als vor $* \ddot{a}$. Im Tscheremissischen jedoch ist das $*_{e}$ der ersten Silbe gewöhnlich auch vor *ä reduziert worden. Wenn dieser Reduzierungsprozess so zu erklären ist, dass das zweite Glied der Vokalkombination * $e-\partial$, also dẹ lautgesetzlich entwickelte reduzierte Vokal der zweiten Silbe, den vorhergehenden Vokal assimilierte (vgl. FUF XXIX 254), so hat dieser Prozess 
verständlicherweise in den $* e$-Stämmen begonnen, in denen der Vokal der zweiten Silbe deutlich früher reduziert worden ist als ${ }^{*} a,{ }^{*} \ddot{a}$ in entsprechender Stellung. So sehen wir denn im Tscheremissischen in der ersten Silbe der ursprünglichen $e$-Stämme als Vertretung von vortscher. ${ }^{*} e$ regelmässig einen reduzierten Vokal, wo ein solcher im Rahmen des tscheremissischen Vokalsystems überhaupt möglich ist. Aber in dieselbe Entwicklung wurde später auch das *e der*ä-Stämme hineingezogen, ein Prozess, der durch die Reduktion des hinter ihm stehenden * $\ddot{a}$ bedingt wurde; nur in der Minderheit der Beispiele blieb das $*_{e}$ von der Reduktion verschont. Möglicherweise trugen hier einige inlautende Konsonanten, namentlich $r$ und $t s ̌$, zur Erhaltung des *e als Vollvokal bei.

d) Ein paar Wörter, in denen das urtscher. $e$ entweder auf ein früheres $* e$ oder auf $* \ddot{a}$ zurückgeht (die ursprüngliche Qualität des Vokals der ersten Silbe lässt sich nicht genau bestimmen, weil wir diese Wörter weder im Ostseefinnischen noch im Mordwinischen antreffen): lè.pa, lep (299), se•rmats,

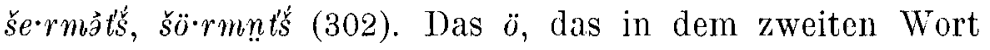
im Osttscheremissischen weitverbreitet ist, erklärt sich aus dem Einfluss des $r$, vielleicht zum Teil auch des $\breve{s}$ am Wortanfang.

e) Auf welchen ursprünglichen Laut das $e$ von $e \cdot r \gamma \grave{a}$ (435) zurückgeht, ist schwer festzustellen. Im Finnischen steht zwar in dem entsprechenden Wort $\ddot{u}$, aber das kann auf einer vom $r$ veranlassten sporadischen Labialisierung oder auf der Einwirkung des gleichbedeutenden Wortes ylliä beruhen. Vom Standpunkt des Tscheremissischen liesse sich leichter die Entwicklung *ir- > er- annehmen als $* \ddot{u r}$ - > er-. Wenn man von $* i$ oder $* \ddot{u}$ ausgehen muss, handelt es sich jedenfalls hier um ein ganz zufälliges Offenwerden eines geschlossenen Vokals vor $r$; aber das $e$ kann auch ursprünglich und das finnische $\ddot{u}$, wie gesagt, sekundär sein.

10. W $\mathrm{O} \ddot{o}$.

Beispiele hierfür haben wir etwa 25, davon bei Beke Cs. Ny. 22-23 20. Weitere Beispiele sind: lötť̌em 'quellen, schwellen', 5-Finnisch-ugrische Forschungen 
mörtnöo 'Rogen', nörə̀ 'biegsam', šörßö 'Esche'. In tschuwassischen Lehnwörtern findet sich ö ungefähr zehnmal.

Das Auftreten des $\ddot{o}$ ist insofern bemerkenswert, als in den meisten eigenen und in den aus dem Tschuwassischen entlehnten gemeintscheremissischen Wörtern unmittelbar auf diesen Vokal $r$ folgt. Beispiele dafür gibt es reichlich 25. R̈̈säNEN, der auf die überragende Stellung der Verbindung -öraufmerksam gemacht und darauf hingeweisen hat, dass vor $r$ kein $\ddot{u}$ anzutreffen ist, schlussfolgert: "Auf Grund dessen bin ich geneigt anzunehmen, dass das Gemeintscheremissische keinen $\ddot{o}$-Laut besass, sondern dies unter Beeinflussung des folgenden $r$ aus $* \ddot{u}$, möglicherweise auch aus anderen Vokalen entwickelt hat" (Tschuw. Lehnw. 97). Es ist wahrscheinlich, dass das $\ddot{o}$ ursprünglich auf diese Weise infolge der die Artikulationsöffnung erweiternden Wirkung, die das $r$ hier wie so oft auch sonst auf den vorhergehenden Vokal ausgeübt hat, aus $\ddot{u}$ entstanden ist. Besondere Hervorhebung aber verdient der von Räsänen erwähnte Umstand, dass offenbar auch einige andere Vokale ausser $* \ddot{u}$ zur Entwicklung der $\ddot{o}$-Kategorie beigetragen haben. Das $\ddot{o}$ scheint seinerzeit sozusagen durch das nachfolgende $r$ bedingt gewesen zu sein und auch andere Vokale als ${ }^{*} \ddot{u}$ entwickelten sich vor $r$ leicht zu $\ddot{o}$. Vgl. weiter unten S. 261. ö tritt in annähernd 10 Wörtern vortscheremissischen Ursprungs auf. Von ihmen ist ungefähr die Hälfte ursprünglich vorderrokalisch und die Hälfte hintervokalisch.

a) Ein ursprüngliches $* \ddot{u}$ dürfte das $\ddot{o}$ in dem Wort šör (425) vertreten. Im Urtscheremissischen hat anscheinend eine Entwicklung *-ür- $>$-ör- stattgefunden.

b) Auf ein vortscher. ${ }^{*} e$ geht das $\ddot{o}$ in $\ddot{o} \cdot r \delta \partial \ddot{z}(348)$ zurück, möglicherweise auch in šöron̉à (350). Im Urtscheremissischen ist bei diesen Wörtern die Entwicklung *-er- $>$-ör- anzunehmen.

Entweder *-ür- $>$-ör- oder *-er- $>$-ör- hat in pört 'Haus' stattgefunden, vgl. fi. pirtti, karel. pertti 'Stube'. Es ist nach wie vor unklar, ob das finnische Wort baltischer (vgl. lit. pirtis) oder slawischer (vgl. russ. nepmb $<$ nbpmb) Herkunft ist, und Gleiches gilt auch für tscher. pört. Dieses ist 
jedenfalls sicherlich älter als das gewöhnliche aus dem Russischen stammende Wortgut der tscheremissischen Sprache, das die jüngste Schicht des tscheremissischen Lehnwortschatzes bildet.

c) Auf vortscher. ${ }^{*} a$ geht das $\ddot{o}$ in den Wörtern $m \ddot{o r}, m \grave{o} \cdot r \grave{\partial}$

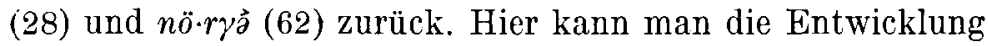
${ }^{*} a>$ urtscher. ${ }^{*} a>*_{o}$ (vgl. S. 194) und eine darauffolgende sporadische Palatalisierung des Vokals zu $\ddot{o}$ annehmen.

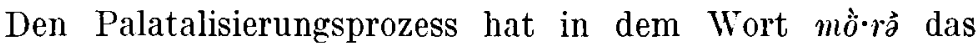
früher am Anfang der zweiten Silbe befindliche $j$ veranlasst $\left(<^{*} m \sigma^{\cdot} \cdot r j a \hat{)}\right.$, bei $n \ddot{o} \cdot r \gamma \dot{\partial}$ wiederum das früher am Wortanfang stehende ${ }^{*} \dot{n}-\left(<{ }^{*} \dot{n} o \cdot r \gamma \hat{\partial}\right)$.

d) $\ddot{o}<$ vortscher. ${ }^{*} o$ in dem Wort $n \hat{o} \cdot r \partial \partial$ (104). Die Entwicklung ${ }^{*} n o \cdot r j a ̂ \partial>n \hat{o} \cdot r \grave{\partial}$ ist ebenso wie in dem ebenerwähn-

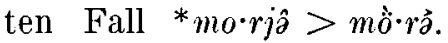

e) $\ddot{o}<$ vortscher. ${ }^{*} \bar{o}$ in $\mathrm{O} n \dot{\hat{o}} \cdot l \grave{\partial}(157)$. Dieses Wort hat wie das obenerwähnte $n \ddot{o} \cdot r \gamma^{\prime}$ ein ${ }^{*} \dot{n}$ gehabt; die ältere urtscher. Form lautete also ${ }^{*} n \dot{n} \cdot l \hat{\partial}$.

Erst im jüngsten Teil des Wortschatzes der tscheremissischen Sprache, d.h. in den tatarischen und russischen Lehnwörtern sowie in den deskriptiv-onomatopoietischen und sonstigen nach der urtscheremissischen Zeit aufgetretenen Elementen ist das Vorkommen des $\ddot{o}$ unabhängig von der Qualität des darauffolgenden Konsonanten.

\section{WOi.}

Beispiele hierfür gibt es etwa 45, von denen BEkE Cs. Ny. 17-19 ungefähr 35 aufzählt. Ausser diesen seien erwähnt: iksa 'Bucht', imà 'Nadel', ǐ̌̀ 'erst, jetzt, erst', liam 'kalben, lammen', liš- 'nahe', nialtem 'streichen', ninə̀ 'diese (Pl.)', pikšs 'Pfeil', $t i$ 'Laus', tištż 'Hausmarke, Namenszeichen'. In den tschuwassischen Lehnwörtern kommt $i$ etwa zehnmal vor. In den späten deskriptiven Wörtern usw. scheint dieser Laut nicht sehr beliebt zu sein.

Urtscher. $i$ muss man ausser in solchen Wörtern, bei denen in allen Dialekten $i$ auftritt, auch in solchen voraus- 
setzen, bei denen das Westtscheremissische und die Mehrzahl der osttscheremissischen Mundarten diese Vertretung aufweisen. Wörter, bei denen in einigren östlichen Mundarten ausnahmsweise $\hat{a}(\hat{\imath})$ steht, sind u.a. ii ( $\sim \mathrm{CKr}$. . în $)$ 'Eis', kiša $(\sim \mathrm{C}$ B kâša) 'Spur', kit $(\sim \mathrm{C}$ kât $)$ 'Hand', lǐs- $(\sim \mathrm{B}$ lăšs $)$

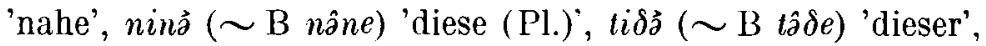
tištà̃ ( C auch tâštâ) 'Hausmarke, Namenszeichen', tit ts' $\left(\sim \mathrm{CKr} . t \imath t \dot{s}, t i t t^{\prime}\right)$ 'voll'. Wir bemerken, dass eine sporadische Neigung zur Reduktion des $i$ eigentlich in zwei Dialektgebieten, Carevokokšajsk und Birsk, anzutreffen ist.

Trotz seiner verhältnismässig geringen Frequenz kommt $i$ in vielen Wörtern von fi.-ugrr. Ursprung vor:

a) $i<$ vortscher. ${ }^{*} \ddot{a}$ in $15-16$ Fällen, die sich in zwei Gruppen gliedern. In der ersten folgt oder folgte unmittel-

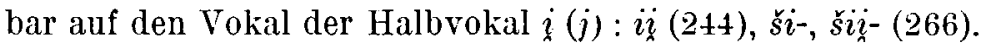

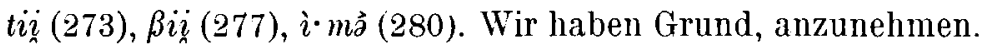
dass die Entwicklung $* \ddot{a}>>i$ wesentlich eine Folge der assimilierenden Wirkung dieses - zum Teil ursprünglichen. zum Teil erst im Tscheremissischen entstandenen - halbvokalischen Elements ist. In den Wörtern der zweiten Gruppe folgt auf das $i$ ein Dentalkonsonant, meist $\check{s}$ : $̌ s \cdot$ štà (240),

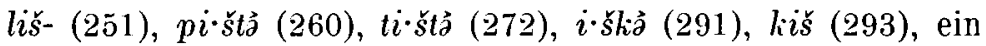
paar Mal $t(\delta)$ : kit $(249)$, $t \grave{\imath} \cdot \delta \dot{\partial}(27 t)$, ? $\beta i \cdot \delta e m$ (343), einmal $n$ : nìn (258). Man kann wohl behaupten, dass die Entwicklung ${ }^{*} \ddot{a}>>i$ vor Dentalkonsonanten lautgesetzlich ist (nicht jedoch vor den Liquidae $l$ und r, s. SS. 210, 211); eine Assimilationswirkung liegt auch hier vor. Da; Wort titšs (276) gehört nach seinem heutigen Lautbestand zur zweiten, aber historisch wahrscheinlich zur ersten Beispielgruppe. Es geht auf die Form ${ }^{*} t i t$ zu:ück, deren $t$ sich kaum anders erklären lässt. als dass es aus der Verbindung -it- der früheren Form *tït enstanden ist. Dies dem Vokal der ersten Silbe folgende $*_{i}$ wiederum dürfte eine ebensolche Entwicklung $v>\ddot{u}>i$ voraussetzen, wie sie in est. täis 'voll' stattgefunden hat.

b) $i<$ vortscher. ${ }^{*} e$. Es gibt hierfür drei oder vier Beispiele (bei zwei von ihnen scheint eine Entwicklung ${ }^{*} n>{ }^{*} n>^{*} i(j)$ nach dem Vokal der ersten Silbe vor sich gegangen zu sein): miem (B PAAs. mijem) (319) und pii (325). Der reduzierte 
Vokal, der in der ersten Silbe der neben pii erscheinenden Form

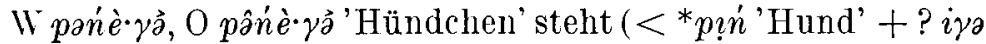
'Junges'; vgl. PaAsonen Wb 93, anders Wichmann JSFOu XXX, 6 S. 8 und Beke A Magyar Tudományos Akadémia nyelv- és irodalomtudományi osztályának közleményei III 63 -64), erscheint wohl als eine ältere Vertretung des ursprünglichen ${ }^{*} e$ als das $i$ in pii. Wenn es sich so verhält, haben wir in pii einen Beweis dafür, dass erst der Wandel des auf den Vokal folgenden $\dot{n}$ zu $i$ die Entwicklung $*_{\partial}\left({ }^{*} ?\right)>i$ veranlasst hat. Anderseits müssen wir uns daran erinnern, dass mundartlich (JU M B) die vollvokalische Form piǹ̀ anzutreffen ist. Da jedoch in den Dialekten $M$ und $B$ die Entwicklung eines reduzierten Vokals zu $i$ eine gewöhnliche Erscheinung ist, dürfte in diesem Zusammenhang nur die Form JU piñè $\gamma$ a von Bedeutung sein, die sich, da sie in einem engen Gebiet auftritt, hinsichtlich des Vokals der ersten Silbe am besten als Analogie zu pii erklärt. - Die übrigen Fälle, die die Entwicklung $*_{e}>i$ aufweisen, sind ? Bidem (343; dies Wort kann allerdings auf die Form *iätä- zurückgehen, worauf auch die mokschamordwinischen Folmen mit ihrem $\varepsilon$ hinweisen; $O \beta \ddot{u} \delta e m$ ist ein Ergebnis einer durch das $\beta$ veranlassten sporadischen Labialisierung) und ßištà (339). Wir stellen hier lediglich fest, dass diese beiden Wörter ursprüngliche $\ddot{a}$-Stämme sind, in denen das $e$ der ersten Silbe bessere Möglichkeiten gehabt haben dürfte, von der Reduktion verschont zu bleiben, als in den ursprünglichen $\varepsilon$-Stämmen, vgl. SS. $212-213$.

c) Auf ein ursprüngliches ${ }^{*} \bar{e}$ geht das $i$ wahrscheinlich in dem Wort liam (354) zurück. Eine interessante grundsätzliche Frage ist es, ob auch bei diesem ursprünglich einsilbigen Vokalstamm der Stamm liin, der einen $i$-Diphthong enthält, gegenüber dem monophthongischen Stamm $l i$, li der ursprüngliche ist. In den vorhin behandelten Fällen erscheint es als natürlich, $i$ für älter als $i$ zu halten, weil das $i$ in ihnen anstelle eines $j$ oder sonstigen Konsonanten steht, der nachweislich in den betreffenden Wörtern gewesen ist. In dem Stamm lii hingegen ist das $i$ eine Erscheinung, deren Wurzeln, soweit bekannt, nicht bis ins Vortscheremissische zurück- 
reichen; es dürfte also als analogisch zu betrachten sein. Vorläufig scheint es sich schwer entscheiden zu lassen, ob sich das $i$ so früh entwickelt hat, dass es den Wandel des vorhergehenden Vokals zu $i$ förderte, oder ob es erst nach dem in der ersten Silbe erfolgten Lautwandel ${ }^{*} \bar{e}>i$ entstanden ist.

d) Für $i<$ vortscher. ${ }^{*} i$ gibt es zwei Beispiele: $i i$ (366) und piža $a \cdot m$ (382). Im ersten Wort ist das $i$ vor $i(<* k)$ vollkommen verständlich. Im zweiten Wort ist das $i$ überraschend; dagegen würde der reduzierte Vokal, der stattdessen in einigen Carevokokšajsker und Birsker Mundarten auftritt, den Erwartungen entsprechen, ist aber in Anbetracht seines engen Vorkommensgebietes offensichtlich sekundär. Wenn pâšte $m$ (382) eine Ableitung vom Verbum $p i z a x \cdot m$ ist, wofür eine grosse Wahrscheinlichkeit besteht, so hat es eine normale Lantvertretung, da es einen urtscher. reduzierten Vokal enthält (vgl. S. 240). Der Typ pi $i$ štem wiederum, der den ursprünglichen nichtreduzierten Vokal aufweist und, soweit bekannt, nur im westtscheremissischen $\mathrm{K}$-Dialekt vorkommt, würde auf der Analogie von $p i \cdot z \ddot{a} m$ beruhen.

e) $i<$ vortscher. ${ }^{*} i$ in dem Wort nii (403). Es wäre ein natürlicher Gedanke, dass tscher. $i$ die normale Vertretung von urspr. ${ }^{*} \bar{\imath}$ geworden wäre. Da jedoch $*_{\imath}$ ein seltener Laut gewesen ist, für den es auch im Tscheremissischen insgesamt nur etwa drei Beispiele gibt, lässt es sich unmöglich feststellen, ob eine solche Tendenz vorhanden gewesen ist. Auch das $i$ von $n i i$ beweist nichts, weil $i\left(<^{*}{ }_{n}^{\prime}<{ }^{*} n\right)$ darauf folgt, durch dessen assimilierende Wirkung das $i$ wie in pii und anderen Wörtern entstanden sein könnte.

f) $i<$ vortscher. $*^{*} \ddot{u}$ in dem Wort $i \cdot k t \grave{\partial}$ (433). Schon die Delabialisierung des $* \ddot{u}$ ist eine seltene, wenn auch nicht ganz alleinstehende Erscheinung. Noch merkwürdiger ist das Auftreten des aus dem $*_{\ddot{u}}$ entstandenen Vokals als Vollvokal. Wenn man hier nicht eine Wirkung des vollen $o$ in dem Zahlwort $k o \cdot k t a \hat{t}$ 'zwei' annehmen kann, bleibt das $i$ des in Rede stehenden Wortes völlig rätselhaft.

g) $*_{u}$ (möglicherweise $*_{0}$ ) wird durch das $i$ des Wortes ija. $m$ (211) vertreten. Hier haben wir ein anschauliches Beispiel dafür, eine wie starke assimilierende Wirkung ein $j(i)$ sporadisch auf den vorhergehenden Vokal hat ausüben können. 


\section{2. $\mathrm{W} i \sim \mathrm{O} e,(\mathrm{JU} \mathrm{CMU}) i$.}

Zu dieser Gruppe dürften 12-15 Wörter gehören, von denen BEкE ('s. Ny. 16 ungefähr die Hälfte aufzählt. Einige

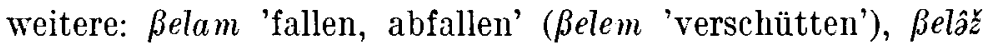
'keilförmiges Seitenstück im Hemd und im Rock', meż 'Wolle'. Da das $i$ der CMU-Mundart ein Zug ist, der im osttscheremissischen Gebiet ausserhalb der Dialektgruppe von JaranskUržum im allgemeinen nicht vorkommt, seien hier alle von dem betreffenden Gewährsmann angegebenen Wörter genannt, die diese Vertretung aufweisen. Es verdient Beachtung, dass der Gewährsmann bei einigen Wörtern auch die Form mit $e$ kannte; er hat nach seiner eigenen Angabe an der Grenze zweier Dialekte gewohnt. Die Beispiele sind folgende: Bile $\cdot s$ (3. Pers. Sg. Präs.) 'abfallen' ( $\beta i l e \cdot m$ 'verschütten'), $\beta i \cdot l \grave{z} z$ 'keilförmiges Seitenstück', $\beta i \cdot m e$ 'Mark', $\beta i \cdot \eta e$ 'Schwieger-

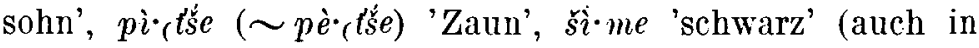
Malmyž und Birsk $i$ : śì $\cdot m a$, šime), šin 'Zunder', š̀ $\cdot r e$ 'ungesalzen, süsslich'. Lediglich in der $e$-Form sind aufgezeichnet worden leßà (3. Pers. Sg. Präs.) 'warm werden, schmelzen' und ter 'Schlitten'; es ist jedoch recht wahrscheinlich, dass der Gewährsmann von diesen Wörtern auch die Formen mit $i$ kannte. Zu einem anderen Tỵp hingegen dürften (CMU) $p e \cdot \eta g_{\partial} \partial e$ 'stark', peš 'sehr' gehören, denn sie haben auch in der nördlichen Dialektgruppe des Westtscheremissischen $e$; die Vokalvertretung der entsprechenden Formen in $\mathrm{K} p i \cdot \eta^{-}$

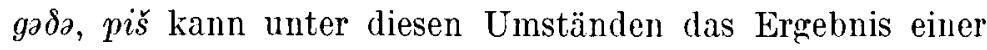
späten Entwicklung $e>i$ sein. - In den tschuwassischen Lehnwörtern treffen wir das in Rede stehende Lautverhältnis etwa fünfmal an. Aus der CMU-Mundart ist $i$ in folgenden Wörtern aufgezeichnet worden: $i r(\sim e r)$ 'Morgen', sir $(\sim$ şer $)$ 'Ufer', sirire $m(\sim$ sere $\cdot m)$ 'schreiben'.

Dieses Lautverhältnis lässt sich mit dem oben in Kapitel 5 behandelten Verhältnis $\mathrm{W}(\mathrm{K}) \boldsymbol{v},(\mathrm{J} \mathrm{V}) u \sim 0 u$ insofern vergleichen, als die kleine Zahl der Beispiele uns daran hindert, als Ausgangsform ein *e oder einen anderen von $e$ und $i$ verschiedenen Laut anzunehmen. Wir haben also die Alternative: entweder urtscher. ${ }^{*} e>W O$ (JU CMU) $i$ oder umgekehrt urtscher. ${ }^{*} i>0$ e. Für die zweite Möglichkeit spricht. 
erstens das Vorkommen der $i$-Vertretung sowohl in westals auch im osttscheremissischen Dialektgebiet. Zweitens scheinen die Konsonantenverhältnisse der in Rede stehenden Wortgruppe in dieselbe Richtung zu weisen. Man kann sich dem Eindruck nicht entziehen, dass das Lautverhältnis W $i \sim \mathrm{O} e$ meistens vor bestimmten Konsonanten auftritt. Nahezu die Hälfte aller Fälle, 7 Wörter, wenn man die eigenen und die aus dem Tschuwassischen entlehnten zusammenzählt, sind solche, in denen auf den Vokal $r$ folgt; dreimal treffen wir $m$ an und zweimal $l$. Betrachten wir nun, wie die Frequenz von urtscher. $e$ und urtscher. $i$ vor diesen drei Konsonanten ist. $e$ findet sich vor $r 16 \mathrm{mal}$, vor $m 7 \mathrm{mal}$ und vor $l 16$ mal. Hingegen dürfte es für gemeintscheremissisches $i$ vor $r$ kein einziges sicheres Beispiel geben, ausgenommen einige ganz junge Lehnwörter, vor $m$ treffen wir es zweimal an, vor $l$ wiederum kein einziges Mal. Es fällt unbedingt leichter, zu erklären, dass das $i$ im Osttscheremissischen die Neigung gehabt hat, sich vor diesen Konsonanten zu $e$ zu öffnen, als den umgekehrten Lautwandel im Westtscheremissischen anzunehmen. Denn wie hätte sich im letztgenannten Fall im Westtscheremissischen trotz des Lautwandels $* e>i$ anderseits das $e$ gerade vor diesen selben Konsonanten so ungemein zahlreich erhalten können? (Vor allen anderen Konsonanten ist die Frequenz des $e$ merklich geringer.)

Wenn diese Erklärung richtig ist, vertritt also das Lautverhältnis $W i \sim O e$ urtscher. ${ }^{*} i$ und die Wörter mit dieser Lautvertretung gehören zur selben Gruppe wie die oben in Kapitel 11 behandelten Wörter. Dann beläuft sich die Gesamtzahl der eigenen urtscheremissischen $* i$-Wörter auf etwa 60. Wir erhalten nun einigen zusätzlichen Aufschluss über die Geschichte des in Rede stehenden Vokals.

a) Von vortscher. ${ }^{*} i$ muss man erstens bei dem Wort pi $\cdot$ tša, $p \grave{e} \cdot\left(t^{\prime} \grave{\partial}\right.$ (375) ausgehen. Aller Wahrscheinlichkeit nach liegt die Entwicklung vortscher. ${ }^{*} i>$ urtscher. $*_{i} i$ auch in $s^{2} \cdot m a$, $\zeta \grave{e} \cdot m \grave{a}$ (402) vor. Vorausgesetzt, dass die Prädikativform $\check{s} \grave{\imath} \cdot m \partial, \breve{s} \grave{e} \cdot m \grave{\jmath}$ gegenüber der einsilbigen Attributform $\check{s} i m$, šem primär ist, so kann man eine Urform *simä (*sima) rekonstruieren, die auch dem entsprechenden syrjänischen 
Wort genau entspricht. - Wie wir weiter unten sehen werden, ist vortscher. ${ }^{*} i$ im Tscheremissischen fast immer reduziert worden. Der Umstand, dass dies in den zwei ebenerwähnten Wörtern nicht geschehen ist, kann damit zusammenhängen, dass sie ursprünglich in der zweiten Silbe den offenen Vokal ${ }^{*} a$ bzw. ${ }^{*} \ddot{a}$ gehabt haben. Dieser wiederum ist später als das in gleicher Silbenstellung befindliche $*_{e}$ reduziert worden und hat dadurch vielleicht zuweilen die Reduzierung des Vokals der ersten Silbe verhindert. Oben ist bereits von t-6 Fällen die Rede gewesen, in denen ursprüngliches * $e$ ebenfalls gerade darum von der Reduktion verschont geblieben zu sein scheint, weil in der zweiten Silbe ein *ä vertretender Vokal gestanden hat, vgl. SS. 212, 217.

b) $*_{i}<$ vortscher. ${ }^{*} \bar{e}$ in dem Wort šin, šen (360).

Unklar ist das $i$ in $\beta \dot{i} \cdot \eta g a, \beta \grave{e} \cdot \eta \grave{\partial}$ (278). Wenn man auf Grund des entsprechenden fi. Wortes vävy ein vortscher. ${ }^{*} \ddot{a}$ annehmen würde, möchte man im Tscheremissischen ein ${ }^{*} \varepsilon$ erwarten. Aber es ist zu beachten, dass auch 1p. vivvâ (im Lappischen geht $i$ in der ersten Silbe normalerweise auf $*_{i}$ zurück, so dass es auch in diesem Wort gut als Entsprechung von tscher. ${ }^{*} i$ passen würde!) und mordw. ov hinsichtlich ihrer Vokalverhältnisse keineswegs mit dem fi. Wort übereinstimmen. Dieser Verwandtschaftsname dürfte zu jenen Elementen der Sprache gehören, mit denen bei verschiedenen Völkern ein zärtlicher Affekt verbunden gewesen ist, was die lautliche Unbeständigkeit erklären würde.

Der Vokal von ßim, ßem (431) lässt sich nicht direkt mit dem $\ddot{u}$ des entsprechenden fi. $y d i n$ vergleichen, weil das tscher. $*_{i}$ hier durch Kontraktion entstanden ist.

\section{W $O \ddot{u}$.}

Hierfür gibt es etwa 50 Beispiele, davon bei BeKE Cs. Ny. 24-26 reichlich 30. Weitere Beispiele sind: jükš 'Schwan', lülam 'Wolle schlagen', nüške 'Eisbrecher', rün 'Reif', rüž (rṇz in der CKr.-Mundart ist deutlich sekundär) 'Lärm', sük, šük 'Kehricht', šükšü 'alt', šültkem 'klopfen, poltern', 
tü- 'das Äussere', tšü,tšem 'bohren, ein Loch machen', tšün (CKr. sekundär t'šnnn( 'Stier'. Wahrscheinlich ist zu den * $\ddot{u}$-Wörtern auch die folgende Wortgruppe zu rechnen, in der man nach Beke in allen Dialekten $\ddot{u}$ antrifft ausser in $\mathrm{K}$ (wo wir ein sekundäres $ә<{ }^{*} n$ finden): šü $t^{\prime} s$ 'Russ', šüyar 'Grab', tüžem 'tausend' (FUF XXIII 81-82). Am ursprünglichsten erscheint das $\ddot{u}$ auch in den folgenden Wörtern, in denen daneben mundartlich $\ddot{o}$ und manchmal $n$ vorkommt: W $l \dot{u} \cdot \delta \partial$, O $l \grave{o} \cdot \delta \vec{z}$ usw. (384), W lü·lpa, O lölpö, lörpö, nörpö 'Erle', W lü·ltem, O

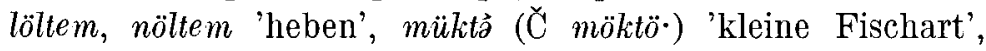

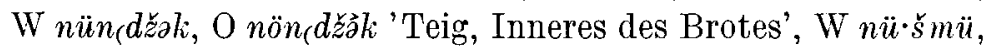
O nöšmə̀ 'Same', W nüštaläm, O nöštâląm 'kneten', W süm, šnm, O šüm, šöm (162), šün ( O šön, šin) (163), s. Beke, Cs. Ny. 23, 26, FUF XXIII 81. In den tschuwassischen Lehnwörtern finden wir urtscher. $\ddot{u}$ knapp zehnmal.

Historisch ist das urtscher. $\ddot{u}$ ganz heterogen. Es lässt sich nachweisen, dass es auf acht verschiedene vortscher. Vokale zurückgeht, von denen keiner sein lautgesetzlicher oder auch nur hauptsächlicher Vorgänger ist. Somit ist das urtscher. $\ddot{u}$ eine auf tscheremissischem Boden aus verschiedenen Elementen entstandene Lautklasse. Es hat sich zweifellos in einer recht frühen Periode herausgebildet, wie aus der Tatsache hervorgeht, dass sich unter den $\ddot{u}$-Wörtern manche solche finden, die zu der fi.-ugr. Wortschicht gehören. Dafür haben wir etwa 25 Beispiele. Die Mehrzahl von ihnen besteht aus Fällen, bei denen sich das $\ddot{u}$ aus irgendeinem Vordervokal als Ergebnis des sporadischen labialisierenden Einflusses der umgebenden Konsonanten entwickelt hat. Es gibt auch einige Beispiele dafür, dass die Palatalisierung eines früheren Hintervokals infolge der assimilierenden Wirkung der umgebenden Konsonanten die Herausbildung des $\ddot{u}$ veranlasst hat. Von diesen Dingen soll in dem Überblick über den Einfluss, den die umgebenden Laute auf die tscheremissischen Vokale ausgeübt haben, näher die Rede sein (S. 246-). Ungefähr die Hälfte dieser ältesten $\ddot{u}$-Wörter sind solche mit einsilbigem auf einen Vokal oder $i$ auslautenden Stamm oder solche, in denen unmittelbar auf das $\ddot{u}$ ein anderer Vokal folgt. Da 
in derartigen Fällen das Auftreten eines reduzierten Vokals nicht möglich ist, kann das $\ddot{u}$ in ihnen in der Tat häufig eine Variante des urtscher. reduzierten $n$ sein, die aus diesem auf Grund einer Art von "Vollvokalisierungs"-Gesetz entstanden ist.

a) $\ddot{u}<$ vortscher. ${ }^{*} e$ in $4-5$ Wörtern: $k \ddot{u} a{ }^{\prime} \cdot m(305)$, lüe्丶 $m$ (314), mülk $k_{s}$ (317), ? müi (320), šüi (334). Mit Ausnahme von

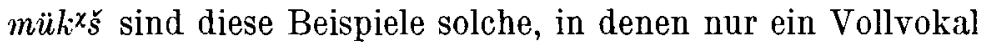
in Frage kommt. Bei den meisten dieser Wörter ist die Ursache der Labialisierung deutlich feststellbar. Bei lüe $\cdot m$ und $s u ̈ i j$. scheint das $\ddot{u}$ aus einer Verschmelzung von ${ }^{*} e$ und darauffolgendem ${ }^{*} v\left({ }^{*} \beta\right)$ entstanden zu sein. Dass $m$ und auch $s$ am Wortanfang eine sporadische Labialisierung verursachen können, ist in Anbetracht der Artikulation dieser Laute natürlich.

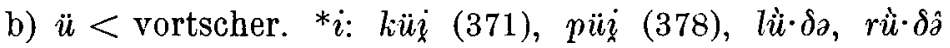
(384). Bei den ersten zwei Wörtern ist nur ein Vollvokal möglich. In dem letzten Beispiel dürfte der unerwartete Vollvokal als Fortsetzer des ursprünglichen ${ }^{*} i$ mit den oben besprochenen Fällen zusammenhängen, in denen das ursprüngliche offene ${ }^{*} a, * \ddot{a}$ der zweiten Silbe durch seine Einwirkung die Reduktion des Vokals der ersten Silbe verhindert zu haben scheint. Wie die der vorigen Gruppe sind auch diese $\ddot{u}$-Fälle Ergebnisse sporadischer Labialisierung, die durch die umgebenden Konsonanten hervorgerufen worden ist. In dem Wort kïi hat sich die Entwicklung ganz ebenso wie in $l \ddot{u} \varrho \cdot m$ und šü $i$ vollzogen; in den übrigen zwei Beispielen ist ein Einfluss des anlautenden Konsonanten anzunehmen. Das Vorkommen von $r$ als labialisierender Faktor wirkt überraschend, aber man kann es wohl nicht bezweifeln, weil es noch andere Wörter gibt, deren Labialvokal es erforderlich macht, zu derselben Erklärung seine Zuflucht zu nehmen.

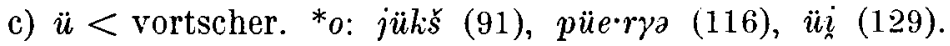
Hier handelt es sich um Palatalisierung, die durch die umgebenden Konsonanten verursacht worden ist. Auch in püe $\cdot r \gamma$ ist früher ein $i(j)$ unmittelbar neben dem Vokal der ersten Silbe gewesen.

d) $\ddot{u}<$ vortscher. ${ }^{*} \ddot{a}:$ : jü $k^{\chi} \tilde{s}_{\ell} \cdot m$ (241), pük $k^{\chi} \breve{s}$ (259). Im zweiten Fall kann man annehmen, dass der Vokal durch den 
Einfluss des anlautenden $p$ labialisiert worden ist, aber im ersten Fall fällt es schwer, eine Erklärung zu geben, wenn nicht möglicherweise der Umstand von Bedeutung ist, dass es unter den $\ddot{u}$-Wörtern mehr als unter den übrigen vordervokalischen Wörtern solche gibt, in denen sich die inlautende Verbindung $k s$ findet. Vielleicht hat die Häufigkeit der Kombination $\ddot{u}+k s$ auch einige solche Wörter mit kš-Verbindung in den Kreis der $\ddot{u}$-Wörter hineinziehen können, in deren erster Silbe sonst irgendein anderer Vokal als $\ddot{u}$ der Erwartung entspräche.

e) $\ddot{u}<{ }^{*} \bar{o}$ : süm, šüm (šnm, šöm) (162), šün (šön, šin) (163). Im ersten Fall hat im Anlaut ursprünglich ein mouillierter Konsonant gestanden, der offenbar die Palatalisierung des Hintervokals veranlasst hat. Was im zweiten Fall die Ursache dieser Entwicklung gewesen ist, dafür dürfte sich keine Erklärung finden.

f) $\ddot{u}<{ }^{*} a$ : $\ddot{u} l-$, $\ddot{u} l \grave{a}-(3), \breve{s} \dot{u} \cdot \delta \grave{z}$ (43). Das an sich durchaus befremdende $\ddot{u}$ in $\ddot{u} l-, \ddot{u} l \grave{\partial}$ - scheint eine natürliche Erklärung zu finden, wenn man darin eine lautliche Einwirkung des Wortes W $\beta a l-$, O $\beta \ddot{u} l-(434 ;<$ urtscher. * $\beta n l-)$ vermutet. Diese Benennungen entgegengesetzter, wichtiger Begriffe sind schon von Anfang an sehr ähnlich geworden, natürlich gerade deshalb, weil man die Abhängigkeitsbeziehung zwischen ihnen stark empfunden hat (man beachte z.B. den bemerkenswerten Wechsel von $a(\ddot{a})$ - und $\boldsymbol{e}$-Stamm bei den zwei entsprechenden finnischen Wörtern: ala $\sim$ ale-, al-; ylï $\sim y l e-, y l$ - sowie die völlige Übereinstimmung in ihrer Flexion). Der Umstand, dass im Tscheremissischen der Unterschied zwischen Hinter- und Vordervokal, der in der ersten Silbe dieser Wörter bestanden hat, auf den kleinstmöglichen Unterschied zwischen dem Vollvokal $\ddot{u}$ und dem reduzierten Vokal ṇ eingeschränkt worden ist, ist eine direkte Fortsetzung jener noch älteren Vereinheitlichungstendenz. Ebenso ist offenbar auch der geschlossene Vokal $i$ zu erklären, den wir im Ostjakischen in der Entsprechung des finnischen Wortes ala antreffen: ostjV $i l$. Anderseits kann das Fehlen des $v$ im Anlaut des urostj. *al, das man mit dem finnischen $y l a ̈$ in Verbindung gebracht hat, auf dem Einfluss des Wortes il 
beruhen. Es besteht gar kein Grund, die Vokale der Stämme ostj. $i l$ und tscher. ül- unmittelbar nebeneinanderzustellen, z.B. indem man in ihnen ein fi.-ugr. ${ }^{*} i$ annimmt, wie es STErNITZ Vok. 58, 98 Fussn. getan hat. - Im tscher. Wort š $\grave{u} \cdot \delta \dot{a}$ hat die Entwicklung $* a>$ urtscher. ${ }^{*} a>*_{o}>*^{*} u>\ddot{u}$ stattgefunden, wobei die letzte Etappe offenbar durch den Einfluss des mouillierten Sibilanten veranlasst worden ist, der früher im Anlaut gestanden hat.

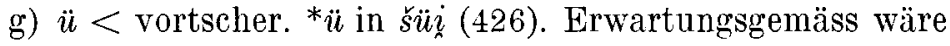
hier $n$ und man kann auch annehmen, dass sich aus diesem in Stämmen dieses Typs lautgesetzlich $\ddot{u}$ entwickelt hat.

h) $\ddot{u}<^{*} \bar{u}$ in jüam (231). Hier handelt es sich um eine durch das anlautende $j$ hervorgerufene Palatalisierung.

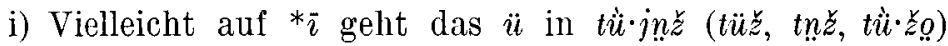
(404) zurück. Die Ursache der Labialisierung ist unklar. rün (407), das als sicheres ${ }^{*} \bar{i}$-Wort wirkt, scheint seinen Labialvokal durch Einwirkung des $r$ bekommen zu haben.

j) Entweder auf $*_{i}$ oder auf $*_{\ddot{u}}$ dürfte das $\ddot{u}$ in $\ddot{u} p^{\varphi} \breve{s}$ (396) zurückgehen. Der Vokal des syrj. is deutet auf $* i$ hin, der des mord. op̀zás wiederum auf $*_{u}$. Falls wir von $*_{i}$ ausgehen müssen, hat das darauffolgende $p$ den Vokal labialisiert.

In den Wörtern W šù $\cdot$ la (386) und šüm (šnm) (420) ist der Vokal durch Kontraktion entstanden. Das zweite der beiden Wörter hat einen Labialvokal gehabt, beim ersten hingegen ist im Tscheremissischen - wahrscheinlich durch den Einfluss des $\check{s}$ am Wortanfang - eine Labialisierung vor sich gegangen.

\section{4. $\mathrm{W} a(\imath) \sim \mathrm{O} \hat{\partial}(\imath), i$.}

Die in diesem Kapitel zu besprechenden Fälle bringt WICHManN Tscher. Texte 126 in zwei Gruppen gegliedert. Er unterscheidet als einen eigenen Typ solche Wörter, in denen er im Osttscheremissischen ausschliesslich $i$ vermerkt hat. Zur anderen Gruppe wiederum gehören nach ihm die Wörter, in denen manche osttscheremissische Mundarten $\hat{\partial}(\grave{\partial})$, manche $i$ haben. Es ist jedoch erstens festzustellen, dass es gar kein solches Lautverhältnis gibt, inm de tscherW a (?) 
in a lle $\mathrm{n}$ osttscheremissischen Dialekten $i$ entspräche. Ferner kann man wohl die Auffassung vertreten, dass wenn $i$ vorkommt, dieses etymologisch betrachtet eine Variante des reduzierten Vokals ist, der in anderen Mundarten stattdessen auftritt. Wenn wir uns in das Problem vertiefen, haben wir Veranlassung, das Forschungsmaterial möglichst ausführlich darzustellen.

Die Gruppe, in der Wichmann Wa $\sim i$ verzeichnet, scheint etwa 15 eigene Wörter zu umfassen: W $\beta a t s, O \beta i t s$,

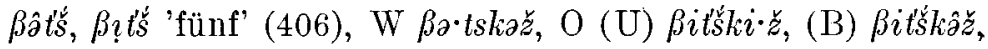

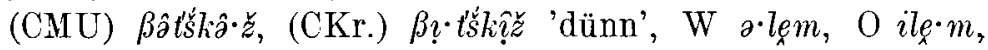

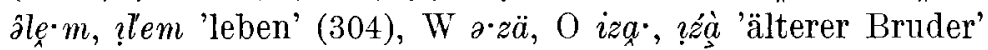

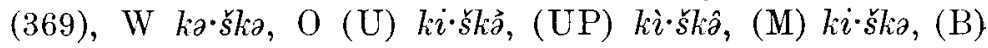
kiške, (CMU) kâ.ške ki.ške, (CKr.) kî.šks 'Schlange', W $k a \cdot$ tskem, O (U) kitške $m,(\mathrm{P} \mathrm{B} \mathrm{M}$ UP) kitskem, (UJ CÜ

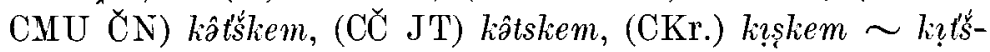
kem 'anspannen', W kə॰tska, O (U) ki $\cdot t^{\prime} k \vec{s},(\mathrm{~B}) k i t^{\prime} k e,(\mathrm{CMU})$ kà.tške, (CKr.) kị̂.şke 'Same, Beere' (PAASONEN Wb ver-

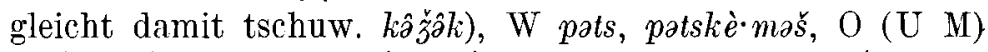

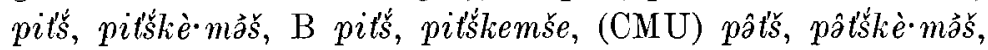
(CKr.) pịśk è mî̌s 'finster' (Wichmann, Tscher. Texte 82 und Paasonen, Wb haben es für möglich gehalten, tscher. pit's mit tschuw. páf(D́žô 'drückend, schwül' zusammenzustellen, aber wenigstens die verschiedene Bedeutung der beiden Wörter lässt es nicht als erforderlich erscheinen, einen derartigen Zusammenhang anzunehmen), W pazə.lmə, O pi $\cdot z l \grave{a}, p i \cdot l^{\prime} z \dot{a}$,

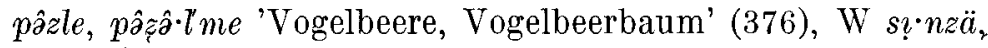

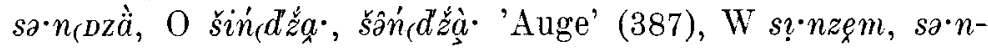
(dzem, O sincd'že $\cdot m$, š

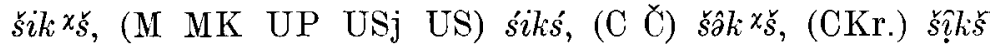

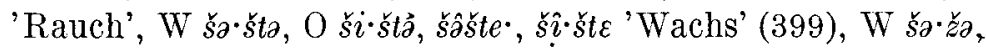

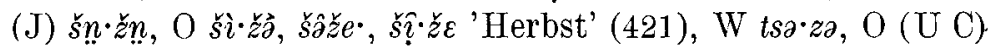

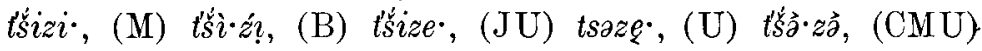

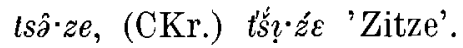

Die Wörter, die nach Wichmann das Lautverhältnis Wa $0 \hat{\partial}(\grave{\partial}), i$ widerspiegeln, kann man in zwei Gruppen einteilen. $\mathrm{Zu}$ der ersten gehören wenigstens folgende 11 Wörter:

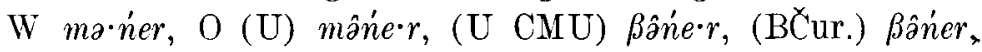




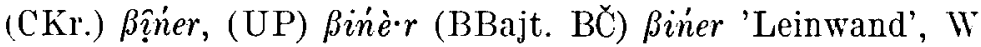

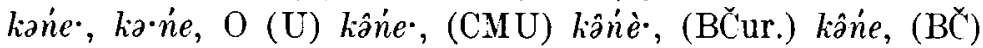
kinè 'Hanf', W kań·läm, O (U) kânela·m, (CMU BČur.) kânelam, (CKr.) kị̂nelam, (BC̈) kinelam 'aufstehen', W ka·ner,

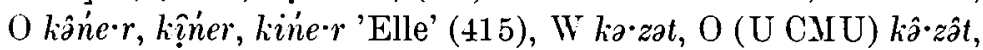

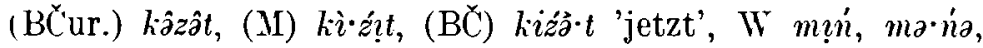

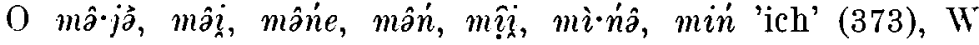

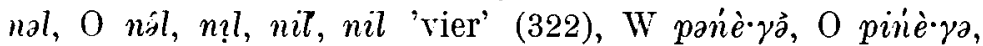

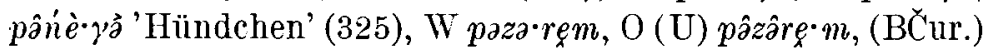

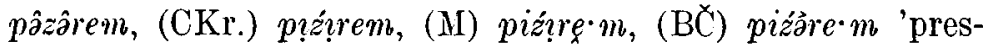
sen' (PAASONEx Wb vergleicht dies Wort mit tschuw. pâ-

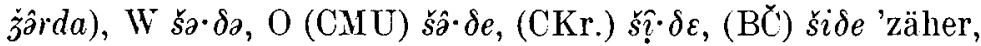
heftiger Charakter ( $=$ fi. sisu); Zorn', W tın', tərñ, O tan',

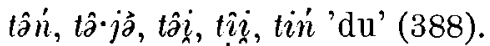

An letzter Stelle folgt die grösste Gruppe: $W^{\infty} j a \cdot \delta \ddot{a} \eta, i \eta n \cdot \delta \ddot{a} \eta$,

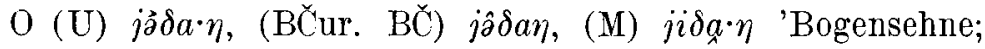
Sehne des Wollschlagbogens', W in l, jal, O (CMU) d'jàl, (BČur.)

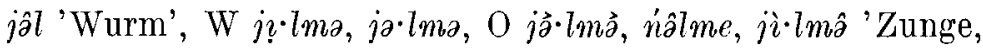

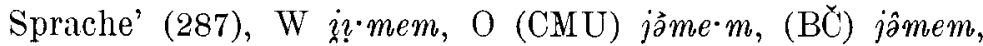
(CKr.) jîmmem 'sich verstecken; gefühllos werden', W jinp $p^{\varphi}$, O (CMU) d'jàpš, (BČur.) jâpšs 'Speer' (288), W jar, O (U CMU)

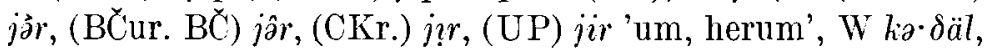

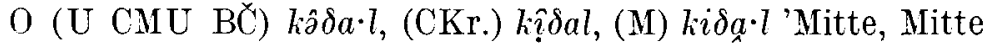
des Leibes', W $k a \cdot \delta e \check{z}, \mathrm{O}(\mathrm{CMU}) k \hat{a} \cdot \delta e \check{z},(\mathrm{P} \mathrm{B}) k \hat{a} \delta \grave{z} z$, (MK UP) kiঠèz 'Zimmer', W kal, O (U CMU BČur. BČ) kâl,

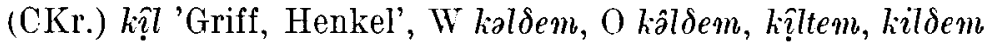
'binden' (347), W $k \cdot \partial \cdot l m \partial, O$ o $k \hat{\partial} \cdot l m \hat{\partial}, k \hat{\imath} \cdot l m \varepsilon, k i \cdot l m \partial$ 'gefroren, erfroren' (408), W kiala·mda, O (CMU) kâlâ.mde, (BČ) $k \hat{\partial}-$

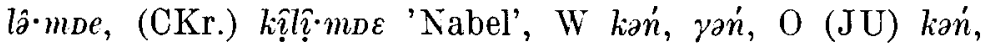

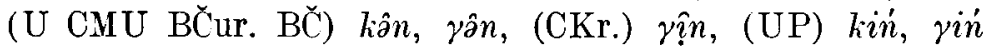
'wenn', W ka•škęm, O (U) kâšskę $m$, (CMU BČ) kâšske m, (CKr.) kị̂skem, (M) kiškęrm 'werfen, schleudern' (395), W kaltšä·kä,

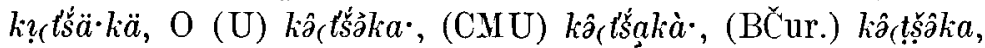

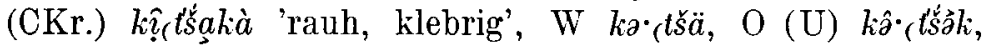

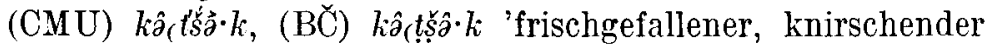
Schnee' (gehört zusammen mit dem vorigen Wort), W kə-

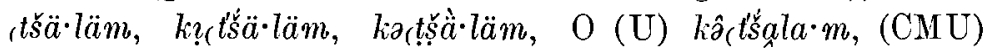

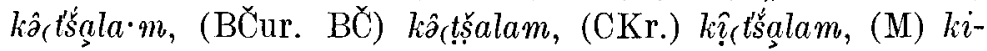




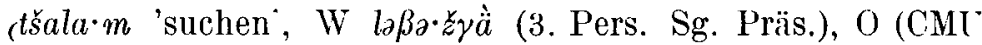

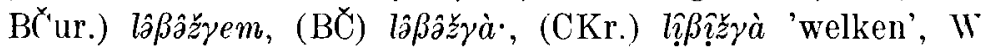

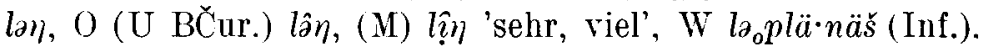
O (BČ) lâplanem, (CKr.) lîplanem 'abnehmen (vom Wind)'. W la•štäš, O lâšta·š, lẹštaš, lištaš 'Blatt' (313), W la·šten. O (U) làštę $\cdot m$, (UP) lištè $\cdot m$, (M) lištę $m$ 'tun, machen', W

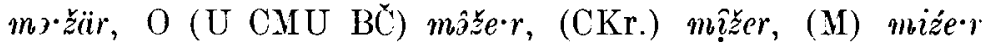
'Kaftan, Rock', W pal, O pâl, p?!, pil' 'Wolke' (379), W pə·laš. pa·lak'x

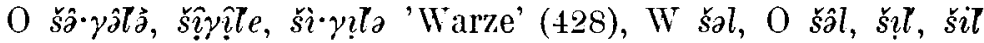
'Fleisch' (400), W ša·läm, O (C.MU BČ) šâla·m, (BČur.) šâlam, (CKr.) šllam, (UP) śilam, (MK) śllam 'sich verstecken', W

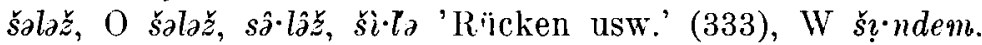
šə·ndem, O šânde·m, ṣ̣̌̂ndem 'stellen, legen' (397), W ša•rpə. O (C.MU BČ) šŝr rpe, (CKr.) š̂̀rpe 'Splitter' (kann aus dem Tatarischen stammen, vgl. Räsäsex Tat. Lehnw. 64), W

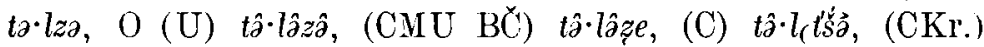

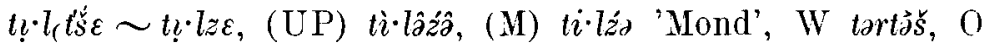
târtâs, (BČur.) târtâka 'Kreisel', W tsaßa.ltẹm, O (U) t'šàßàl-

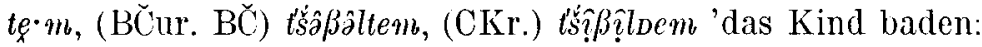

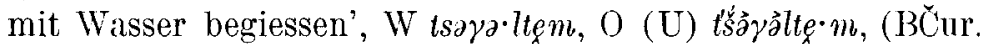

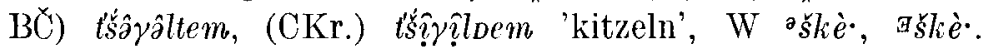

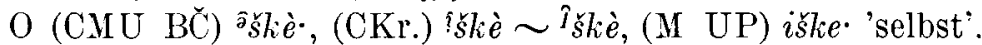
$\mathrm{W}$ əšte $\cdot m, a \cdot s ̌ t e m, \mathrm{O}$ åste $\cdot m, \hat{\partial} s ̌ t e \cdot m$, ?štem 'machen, tun' (303). W a•štar, O (CMU BČur. BČ) ầ štarr, (BČ̌ur. BČ) âštâr, (U) $\hat{\Xi} s ̌ t h \cdot r$, (CKr.) $\hat{I}_{\mathscr{s}} t \hat{\imath} r$ 'Fusslappen, Beinwickel'. Insgesamt etwa 35 Beispiele.

Die e r s t e der obigen drei Beispielgruppen kann man folgendermassen charakterisieren: Im Westtscheremissischen haben wir $\partial$, mundartlich manchmal $\ell$, im Osttscheremissischen $i$ u.a. in den Dialekten von Sernur und Kupsola im Kreis Uržum (die Wichmasx erforscht hat) sowie in den Dialekten von Malmyž und Birsk, aber einen reduzierten Vokal $(\hat{\partial}, \hat{\imath}, \imath)$ u.a. in den Mundarten von Jaransk-Uržum, Carevokokšajsk und Čeboksar sowie anscheinend auch in einigen Uržumer Dialekten. Unter den hierher gehörenden Wörtern befinden sich fünf, in denen auf den Vokal der ersten

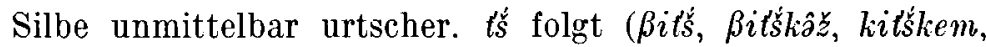


$k i \cdot t^{\prime} k \hat{z}$, pit'ś), in drei Wörtern folgt auf den Vokal $z(\dot{z}, z)$

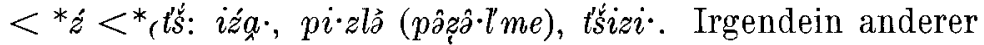
mouillierter urtscher. Konsonant ist nach dem Vokal in den

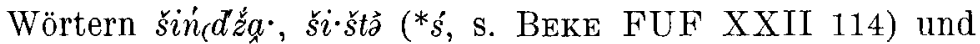
$\check{s} \imath \cdot{ }^{2} \vec{z}\left({ }^{*} z<* \dot{s}\right.$, s. Beke a.a.O.) gewesen. Ein mouilliertes ${ }^{*} \dot{s}$ kann man mit gutem Grund auch in $k i \cdot s ̌ b s$ annehmen; man beachte vor allem das $s$ des UP-Dialekts (urspr. nichtmouilliertes ${ }^{*} s$ wird in diesem Dialekt in vordervokalischen Wörtern durch $s$ vertreten). Übrig sind noch die Wörter ile $m$ und šik $x_{s}$. In ersterem ist auf den Vokal der ersten Silbe möglicherweise im Urtscheremissischen ein mouillierter Konsonant gefolgt

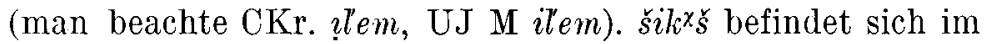
Vergleich zu allen anderen Wörtern dieser Gruppe insofern in einer Sonderstellung, als der auf den Vokal folgende Konsonant $k$ sicherlich schon von Anfang an nichtmouilliert gewesen ist; ausserdem deutet die Form siks in M UP darauf hin, dass die beiden $s$-Laute des Wortes im Urtscheremissischen nichtmouilliert waren. Unsere Aufmerksamkeit wird hier auf die Form $\check{s}_{i} k^{\times}{ }_{s}^{x}$ in der Jaransker Mundart gelenkt (Beke, FUF XXII 1.04). In dieser Mundart kann der Vollvokal $i$ auf keinen Fall die normale Entsprechung des a der westtscheremissischen V- und K-Dialekte $\left(\check{s}^{2} k^{\times} s ̌\right)$ und ebensowenig des $\hat{a}$ der osttscheremissischen C- und Č-Dialekte ( $\left.\check{s}_{\hat{z}} k^{\times} \tilde{s}\right)$ sein. Die interdialektalen Vokalverhältnisse dieses Wortes sind also als unregelmässig anzusehen.

In der $\mathrm{z}$ w e i t e $\mathrm{n}$ Beispielgruppe herrscht im Westtscheremissischen die gleiche Vokalvertretung wie in der ersten. Im Osttscheremissischen bemerken wir, dass die $i$-Vertretung weniger verbreitet ist. $i$ erscheint wie auch in der ersten Gruppe durchgehend in einigen Mundarten ron Malmyž, Uržum (UP) und Birsk (BČ). Dagegen haben z.B. der Dialekt von Sernur im Kreis Uržum und der Birsker Curajevaer Dialekt jetzt $\hat{\partial}$. In sechs von den zehn Wörtern, die zu dieser Gruppe gehören, folgt auf den Vokal der ersten Silbe urtscher. n: ßâne•r, kânè $\cdot$, kânela·m, kâne·r, mân (mấn), tân (tâni). In zwei

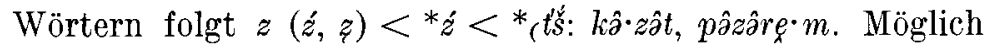
ist auch, dass im Zahlwort nâl im Urtscheremissischen der mouillierte Konsonant $l^{\prime}$ gestanden hat. Vielleicht kann man 
auch in šâ. $\delta e$ ein frühurtscher. ${ }^{*} \delta$ ' annehmen, das dann zu $\delta$ geworden wäre wie beispielsweise in kodem 'lassen'.

Die d ritte Gruppe enthält in der ersten Silbe überall einen reduzierten Vokal ausser in einigen Dialekten des Kreises Malmyž und wenigstens noch in der Mundart des Dorfes Petrušin in Uržum (UP); diese dürften in fast allen Wörtern $i$-Vertretung aufweisen. Den Wörtern dieser dritten Gruppe ist es eigentümlich, dass man kaum in einem einzigen Fall mit Sicherheit einen urtscher. mouillierten Konsonanten nach dem Vokal der ersten Silbe nachweisen kann. Etwa die Hälfte der Wörter - in ihnen folgt auf den Vokal der ersten

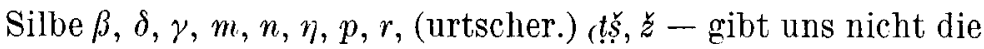
Möglichkeit, einen solchen Konsonanten anzunehmen. Dasselbe können wir von einigen Wörtern sagen, die eine mit $\breve{s}$ beginnende Konsonantenverbindung enthalten und in denen das $\breve{s}$ nachweislich schon vortscheremissisch ist $(l \hat{s} s ̌ t a \cdot s ̌, ~ \partial ̂ s ̌ t e \cdot m)$. Wemn dem Verb lâštę: $m$ das wotj. leśtini 'machen, verfertigen, bereiten, zurichten' mit ursprünglichem $s$ entspricht, so würden wir urtscher. ${ }^{*} l$ lste $\cdot m>\mathrm{U}{ }^{*} l i s ̌ t e q m$ usw. erwarten. In diesem Fall jedoch lässt sich denken, dass der Lautbestand des Verbs von dem Synonym âšte $m$ beeinflusst worden ist. Unregelmässig ist auch ${ }^{2} \breve{s} k \grave{e} \cdot$ 'selbst', falls fi. itse und mordw. $e s ́$ Entsprechungen dazu sind. Vor ursprünglichem *'s wäre eine solche Vertretung zu erwarten, wie sie in der ersten Beispielgruppe anzutreffen ist, also z.B. U BČ *išk $\grave{e} \cdot$ Möglicherweise ist der Vokal der ersten Silbe, da er unbetont war, bereits im Urtscheremissischen zu einem überkurzen Laut geschwächt worden, wie denn solche überkurzen Vokale sowohl im Westtscheremissischen als auch weithin in osttscheremissischen Mundarten in diesem Wort zu finden sind. Man kann die Frage aufwerfen, ob der Vokal sich aus diesem Grunde anders entwickelt hat als in der ersten Gruppe von Beispielen, zu der das in Rede stehende Wort sonst seinem Typ nach gehören müsste. Der normalkurze Vokal anstelle eines überkurzen in der Form iške in M UP wäre dann eine späte, nur auf diesen Dialekttyp beschränkte Erscheinung. Aber wenn man zuletzt in Betracht zieht, dass $\breve{s}$ auch in kâs$k e \cdot m$ ein ursprüngliches ${ }^{*} s ́$ vertreten kann (vgl. syrj. kiślkyny), 
sieht es so aus, als ob vor dem tonlosen Sibilanten in gewissem Masse eine Ausgleichung zu Gunsten der Vokalvariante stattgefunden hat, die sich vor einem ursprünglichen nichtmouillierten Sibilanten gebildet hat. Unklar bleiben 11 Wörter, in denen auf den Vokal der ersten Silbe $l$ folgt. An und für sich gibt es in ihnen nichts, was uns unbedingt zwingen würde, urtscher. mouilliertes $l$ anzunehmen, aber anderseits kann man darauf hinweisen, dass in den meisten Mundarten z.B. pâl,

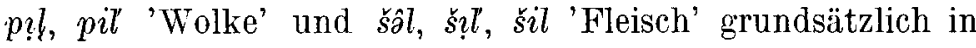
der gleichen Weise vertreten sind wie das zu der zweiten Beispielgruppe gehörende nâl, nı̣l, nil', nil 'vier', bei dem wir urtscher. $l$ für möglich hielten. Auf diese wichtige Frage kommen wir später noch zurück.

Unsere Betrachtung führt zu folgendem Schluss, dessen Berechtigung sich wohl nicht bestreiten lässt, wenn auch einige auf Vermischung oder Ausgleichung beruhende Ausnahmen hier und da sich finden sollten: In allen $\mathrm{drei}$ Gruppen von Beispielen vertritt der Vokalderersten Silbe ein und dasselbe Phonem, nämlich einenurtscher. reduzierten illabialen Palatalvokal. Dieser ist vor einem nichtmouillierten Konsonanten im Urtscheremissischen offenbar *a gewesen, vor einem mouillierten aber *?. Demnach sind $*_{\partial}$ und *? ursprünglich kombinatorische Varianten voneinander, obwohl sie sich später mundartlich in sehr verschiedenen Richtungen entwickelt haben. Besonders dürfte Veranlassung bestehen, hervorzuheben, dass der in vielen osttscheremissischen Dialekten vorkommende reduzierte Mittelvokal erst nach der urtscheremissischen Zeit aus einem Palatalvokal entstanden ist. Die Kategorie der Mittelvokale hat es im Urtscheremissischen überhaupt nicht gegeben.

Im Westtscheremissischen sind $*_{\partial}$ und ${ }^{*} ?$ zu a zusammengefallen. Ausschliessliche $ə$-Vertretung herrscht in den von BEkE erforschten Dialekten der Kozmodemjansker und Jaransker Gruppe, über die uns Angaben zur Verfügung stehen, ebenso wie in dem vom Verfasser erforschten Dialekt von Jatykovo, der zur Kozmodemjansker Gruppe gehört. 
Wichmans und Ramstedt hingegen haben in Kozmodemjansker Mundarten neben a auch in gewissem Grade 2 -Vertretung festgestellt, aber dieses ? ist deutlich eine späte kombinatorische Variante von $ə$, was am besten daraus ersichtlich wird, dass auch aus urtscher. $n$ entstandenes $ə$ an der Entwicklung $a>$ ? teilgenommen hat (z.B. tı $\eta$ 'Stammende, dickes

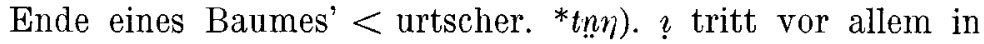
geschlossenen Silben auf, die auf $n, \dot{n}$ oder $\eta$ auslauten. Neben der regressiven Wirkung dieser Nasalkonsonanten kommt auch progressive Assimilation $j^{-}>j \eta^{-}$vor, bei Wichmann nur in geschlossener Silbe (z.B. in.lma 'Zunge, Sprache'), bei Ramstedt auch in offener Silbe (z.B. $i \imath \cdot \delta \ddot{a} \eta$, Wichm. $j ə \cdot \delta \ddot{a \eta}$ 'Bogensehne'). Auf den Vokal folgendes $r$ hat Erhaltung des a veranlasst: Ramst. iar, Wichm. jar 'Kreis; herum'.

Wenn wir nun zum Osttscheremissischen übergehen, betrachten wir zuerst die "mittlere Sprache» von Jaransk-Uržum. Die von Wichmans veröffentlichten, allerdings sehr knappen Aufzeichnungen über die Mundart des Dorfes Upša scheinen zu beweisen, dass hier $\partial^{-V e r t r e t u n g ~ h e r r s c h t, ~ a l s o ~ d i e-~}$ selbe wie im Westtscheremissischen (z.B. pal 'Wolke', $k^{a} n e$ ' 'Hanf', ša·ncDzà [3. Pers. Sg. Präs.] 'wissen'). Im Dialekt von Turšomučakš, den BEkE erforscht hat, sehen wir wiederum ausschliesslich den Mittelvokal $\hat{\partial}$, z.B. '̂lem 'leben', šâl 'Fleisch',

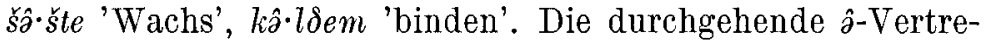
tung reicht vom Jaransk-Uržumer Gebiet bis zu einigen Carevokokšajsker Dialekten, wie der Mundart von Marij Ušem, die beispielsweise die Formen ßôt's' 'fünf', kône.r 'Elle', lâšta·š 'Blatt' vertreten. Ein Sonderfall ist $j \hat{\partial}->j \grave{\partial}-$, z.B. d'jàpš 'Speer'. Wenn im CMU-Dialekt neben $\hat{a}$ oder stattdessen $i$ anzutreffen ist, handelt es sich um Einwirkung der Nachbarmundarten oder der Gemeinsprache.

Die Mundart von Krasnojar stimmt mit dem CMU-Dialekt in der Hinsicht überein, dass auch sie stets einen reduzierten Vokal hat. Es gibt hier jedoch zwei reduzierte Vokale, nämlich das vordere ? und das mittlere ? . Dem Gewährsmann des Verfassers schien der Unterschied zwischen $i, ?$ und $\hat{\imath}$ klar zu sein, aber ein mit der Phonetik dieser Mundart nicht vertrautes Ohr hat es schwer, im Anlaut und vielfach auch vor 
einem mouillierten oder halbmouillierten $l$, manchmal auch vor anderen Konsonanten dieser Art, ? von $i$ zu unterscheiden. Anderseits war auch der Unterschied zwischen ? und ? nicht immer ganz deutlich zu hören - anscheinend je nach den umgebenden Konsonanten. So dürfte sich auch der Umstand erklären, dass der Verfasser in einigen Wörtern einmal $\boldsymbol{\imath}$, einmal ? aufgezeichnet hat. Auch die Möglichkeit ist natürlich in Betracht zu ziehen, dass die Aussprache des Gewährsmannes wirklich schwankte. Leider blieb diese interessante Einzelheit unter den aussergewöhnlichen Verhältnissen, in denen die Sammelarbeit vor sich ging, ohne endgültige Nachprüfung. Wegen dieser möglichen Ungenauigkeiten in den Aufzeichnungen können wir keine sichere Antwort auf die Frage geben, ob das ? des CKr.-Dialektes direkt dem urtscher. ${ }^{*}$ ? und das ? dieses Dialektes dem urtscher. ${ }^{*}$ entspricht. Es kann sich durchaus so verhalten und ursprünglich ist jedenfalls sicherlich urtscher. ${ }^{*} ?>\mathrm{CKr}$. ? und urtscher. ${ }^{*} \partial>$ ? ${ }^{*} \hat{\jmath}>\mathrm{CKr}$. ? (es ist recht glaubhaft, dass das CKr. $\hat{\imath}$, das ja geschlossener ist als die normale $\hat{\partial}$-Vertretung des Tscheremissischen, auf dem Einfluss des ? beruht). Man beachte, dass über die Hälfte der auf S. 226 aufgezählten Wörter der ersten Beispielgruppe nach den Aufzeichnungen $\imath$-Vertretung aufweisen: $\beta \imath t^{\prime} \hat{s}^{\prime}$,

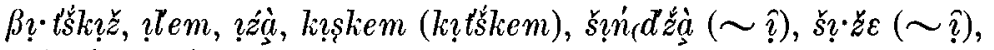

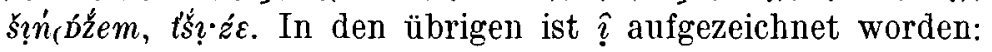

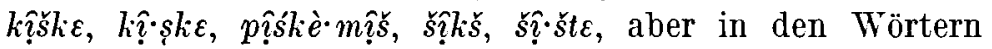
$k \hat{\imath} \cdot s k \varepsilon, p \hat{\imath} s k i \grave{e} \cdot m \hat{\imath} \hat{s}$ ist vielleicht vor dem mouillierten $\dot{s} \mathrm{bzw}$. dem halbmouillierten $s$ in Wirklichkeit ein ?, denn neben dem Wort kişkem (kętśkem), das den gleichen Typ vertritt, ist ausdrücklich vermerkt, dass ? in derartigen Fällen dem ? akustisch nahsteht. - In der zweiten Gruppe von Beispielen ist ausser in zwei Wörtern ( $n$ ?l, p!z?rem) überall ? aufgezeichnet worden und desgleichen fast durchgehend in der dritten, obwohl hier ? vor $l\left(l, l^{\prime}\right)$ ziemlich oft vermerkt ist: $p ? l, p ? l \underline{s}$, ,

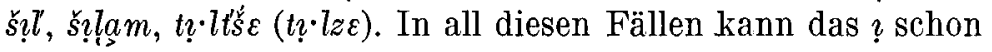
urtscher. ? vertreten.

Der Gedanke, dass $l$ nach urtscher. *? (nur als Einzelkonsonant, nicht als Anfangskonsonant einer Konsonantenverbindung) mouilliert gewesen ist, bedeutet nicht, dass es sich um 
ein auf das Vortscheremissische zurückgehendes oder aus einer früheren *-lj-Verbindung entstandenes $l$ gehandelt haben müsste. Zwar hat es auch derartige Fälle gegeben: $n ?$ l 'vier', p?lıšs 'Ohr' und š? ? 'Fleisch'. Aber wir haben wohl genügenden Grund zu der Annahme, dass ausserdem vortscher. ${ }^{*} i$ die Mouillierung eines darauf folgenden einzelnen $l$ veranlasst hat. Daher dürfte z.B. CKr. pıl 'Wolke' auf die urtscher. Form ${ }^{*} p$ ? l zurückgehen. Ferner lässt sich denken, dass die Lautverbindung - $2 l$ - in manchen Fällen analogisch verallgemeinert und anstelle der Verbindung -al-gesetzt wurde, mit anderen Worten, dass der analogische Ausgleich dazu geführt hat. dass das auf den Vokal folgende $l$ durch die $?$-Vertretung bedingt war, was zugleich antomatisch Mouillierung des $l$ zur Folge hatte. So wäre urtscher. *?l'em 'leben' aus der früheren Form *alem entstanden. Man beachte vergleichshalber. dass vortscher. ${ }^{*} i$ auch darauf folgendes $n$ mouilliert zu haben scheint. So dürften sich am besten u.a. die urtscher. Prono-

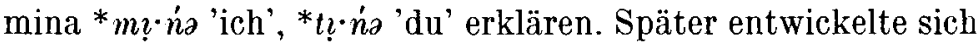
das $l$ in manchen Mundarten zu $l$ (wogegen sich das auf entsprechende Weise entstandene $\dot{n}$ erhielt). Auf Grund des Wor-

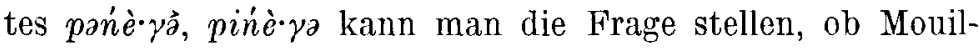
lierung des $n$ auch nach vortscher. ${ }^{*} e$ vorgekommen ist. Vgl. auch mi.em 'kommen, gehen' (s. S. 216), das auf die frühurtscher. Form *m?ne $m$ zurückgehen kann. Oder sollte eine Ausgleichung $-2 n->-\imath n^{\prime}$ - stattgefunden haben, die mit der Entwicklung -al- $>-? l-z u$ vergleichen wäre?

Alles in allem scheinen die Grundzüge der Vertretung im CKr.-Dialekt auf urtscheremissische Prämissen hinzudeuten, wenn auch einige Ausgleichungen vor sich gegangen sind. Die bemerkenswerteste derselben ist die Neigung des ?, sich vor $l$ $\mathrm{zu}$ verallgemeinern.

In der Mundart des Dorfes kühks-nur im Kreis Carevokoksajsk (CK) sowie in den Mundarten der Dörfer kuro-mola-

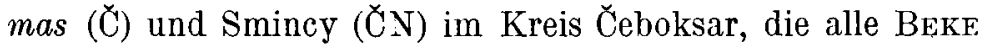
erforscht hat, findet sich im allgemeinen $\hat{\partial}$, aber einige Male

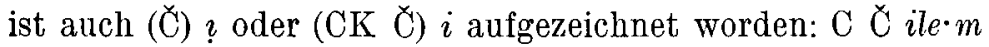
'leben', CK С̆ ßizâmâše 'der fünfte', CK Č nilâmâse 'der vierte',

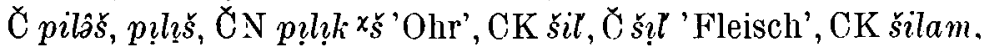


¿ šilam 'sich verstecken; durchgehen'. Alle diese Wörter haben im CKr.-Dialekt ?; somit ist es offensichtlich, dass die

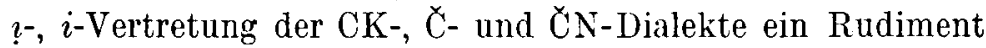
des alten Zustandes ist, der sich verhältnismässig am besten im CKr.-Dialekt erhalten hat. Es fällt auf, dass $\imath, i$ in $\mathrm{CK} \circlearrowright$ $\breve{C} \mathrm{~N}$ am häufigsten vor $l$ aufzutreten scheint. Auch dieser Zug stimmt weitgehend mit der Vertretung in der CKr.-Mundart überein.

Von den soeben betrachteten Mundarten, in denen wir neben ! auch den Vollvokal $i$ antreffen, ist ein recht kurzer Schritt zu dem Dialekttyp, in dem das *? ganz und gar zu $i$ geworden ist. In den Mundarten von Sernur und Kupsola im Kreis Uržum ist * $*^{>} i$ nur in der ersten Gruppe von Beispielen vor sich gegangen (S. 228). In diesem Dialekttyp ist eine starke Tendenz zur Velarisierung des ${ }^{*}$ ? wirksam gewesen, aber bei den in Rede stehenden Wörtern hat sich das ${ }^{*}$ ? seine Palatalität erhalten und später, anscheinend gleichzeitig mit den Veränderungen urtscher. $\approx>u$ und $n>\ddot{u}$, zu $i$ weiterentwickelt. Die Mouillierung des folgenden Konsonanten genïgte allein zur Verhinderung der Velarisierung des * ${ }^{*}$ nur in dem Fall, dass der $\mathrm{K}$ ons o nant in dieselbe Silbe hineingehörte wie das ${ }^{*}$ ? (

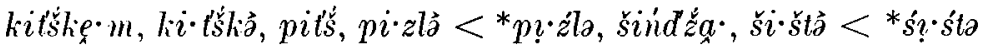

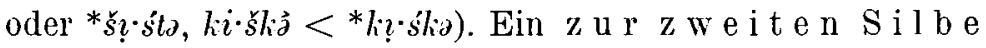
gehörender mouillierter Konsonant bewahrte das * ${ }^{*}$ offenbar nur unter der Bedingung, dass dieses im Anlaut

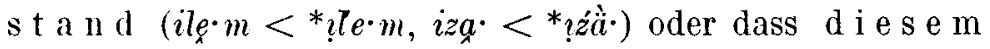
a uch ein mouillierter Konsonant voraus-

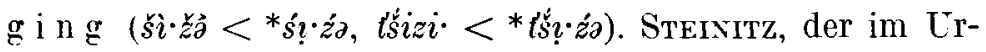
tscheremissischen nur einen einzigen reduzierten Vokal $\left({ }^{*} a\right)$ annimmt, hat Vok. 97 richtig festgestellt, dass die $i$-Vertretung in $U$ sich lautgesetzlich in geschlossener silbe vor einem mouillierten Konsonanten entwickelt hat. Dagegen dürfte sein - mit einem Fragezeichen versehener - Gedanke, dass die gleiche Entwicklung unabhängig von der Qualität des auf den Vokal folgenden Konsonanten auch im Anlaut vor sich gegangen wäre, wohl nicht der Wahrheit entsprechen. Wir haben im Uržumer Dialekt wenigstens vier Beispiele für $\hat{\partial}$ 
$\left(<\right.$ urtscher. ${ }^{*}$ ) im Anlaut vor einem nichtmouillierten Konsonanten, während es für $i$ in gleicher Stellung nur zwei Beispiele gibt. Von diesen kann das $i$ pro ${ }^{*} \hat{\partial}$ in der Desiderativform ing: $m$ des Verneinungsverbs (vgl. $\mathrm{K}{ }^{a}$ ne $m$ ) auf dem Einfluss des sicherlich schon urtscheremissischen $i$ des Imperativs (it, $i \delta \dot{a} \cdot$ ) beruhen. Das $i$ des Zahlworts indè $\cdot s \grave{\partial}$ 'neun'

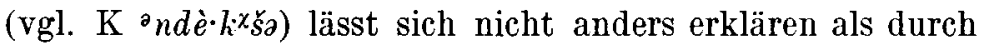
Annahme einer unregelmässigen Doppelheit $*_{\partial} \sim *_{i}$. Man beachte auch $\mathrm{J} n n n d i \cdot \eta \check{s}$, in dem ausnahmsweise $\boldsymbol{n}$ vorkommt. Ein derartiges lautliches Schwanken ist bei Zahlwörtern eine gewöhnliche Erscheinung. - Bei šik $\chi_{s}$ muss man die auf einer unregelmässigen Lautentwicklung beruhenden Parallelformen ${ }^{*}$ saks $\sim{ }^{*}$ sitss annehmen (vgl. S. 229), von denen gerade die zweite sich den Uržumer Mundarten vererbt hat. In allen übrigen Stellungen - also in der zweiten Gruppe von Beispielen (SS. 226-227) - ist das *? velarisiert worden und mit $\hat{a}<$ urtscher. $a$ zusammengefallen. Die Entwicklung $*_{\partial}>\hat{\partial}$ hat lautgesetzlich in den meisten osttscheremissischen Mundarten stattgefunden.

Der von Passones erforschte Dialekt von Curajeva in Birsk hat ziemlich genau die gleiche Vertretung wie der im vorigen Abschnitt behandelte Uržumer Dialekttyp. Ein Unterschied findet sich wohl nur in den beiden folgenden Wör-

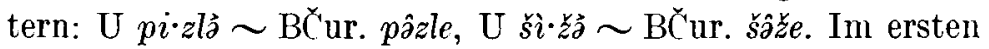

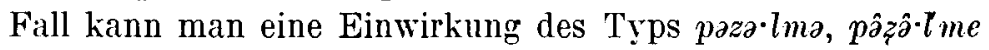
vermuten, in dem das ${ }^{*} ?$ der ersten Silbe sich in der Mundart ron Curajeva lautgesetzlich zu $\hat{a}$ weiterentwickelt haben dürfte, weil der danebenstehende mouillierte Konsonant zur zweiten Silbe gehört. BČur. šâže weist vielleicht auf eine Nebenform hin, die neben der urtscher. Form * ś? $\cdot z$ z vorkam und im Anlaut bzw. Inlaut statt des mouillierten Sibilanten *s bzw. ${ }^{*} z$ oder $* \check{s}$ bzw. $* z$ hatte. Bei Wörtern, in denen ein Sibilant sowohl im Anlaut als auch im Inlaut steht, ist ein derartiges Schwanken gewöhnlich. Ein guter Beweis dafür sind u.a. die Entsprechungen des Wortes šâže in verschiedenen fi.-ugr. Sprachen.

Die Mundart von Caskino in Birsk vertritt einen Typ, in dem urtscher. ${ }^{*} ?>i$ vor einem zur zweiten Silbe gehörenden 
mouillierten Konsonanten, auch dann, wenn im Anlaut ein nichtmouillierter Konsonant steht, also in der zweiten Gruppe von Beispielen (SS. 226-227). In dieser Hinsicht unterscheidet sie sich somit von den oben betrachteten Uržumer und Birsker Dialekten. Wegen der Spärlichkeit des verfügbaren veröffentlichten Materials kann man keine sicheren Schlüsse darüber ziehen, zu welchem von beiden Typen die von Beke erforschte Mundart des Dorfes Starinonenger (tošto-jal) im Kreis Malmyž (M) gehört. Jedenfalls tritt auch hier die Doppelheit $i \sim \hat{\partial}$ auf und das $i$ in nilamase 'der vierte' scheint diesen Dialekt näher an den von Caskino anzuschliessen, der ebenfalls $i$ hat (nil), zum Unterschied von dem $\hat{a}$ ( $n \hat{a} l)$ der Mundarten von Sernur und Curajeva.

Die Lautverhältnisse des in Rede stehenden Zahlworts in den verschiedenen Dialekten geben uns Gelegenheit, die Betrachtung der späteren Geschichte der oben (S. 234) von uns angenommenen urtscher. Lautverbindung ${ }^{*}$ ? $l$ - fortzusetzen. Wenn wir von der urtscher. Form ${ }^{*} n ! l$ ausgehen (vortscher. ${ }^{*} e>{ }^{*}$ ? unter dem Einfluss des darauf folgenden $\left.l<*_{-} l j-\right)$, wäre in den Dialekten von Sernur und Čurajeva gerade nil zu erwarten. Warum finden wir diese Form hier nicht? Die Antwort dürfte die Entmouillierung des $l$ nach ${ }^{*}$ ? geben, eine Erscheinung, die sowohl im Westtscheremissischen als auch weithin im Osttscheremissischen vorkommt und somit zweifellos schon im Späturtscheremissischen angefangen hat. Gerade der Lautwandel $l>l$ scheint die Verschmelzung des *? mit dem *ə gefördert zu haben, die vor einem unmouillierten Konsonanten lautgesetzlich war. So ging also die Entwicklung $*_{-\imath \imath l-}>*_{-}^{*} l->-\hat{l} l$ - vor sich, deren Endpunkt uns die Form U BČur. nâl zeigt. Die Tatsache, dass in der BČ-Mundart in diesem Wort das $i$ erhalten blieb, obwohl auch diese Mundart zu dem Bereich der Entmouillierung des $l$ gehört, erklärt sich am natürlichsten durch die Einwirkung des Zahlworts pit's 'fünf'. Aber wie steht es mit ile $m$ 'leben', in dem wir trotz der Entwicklung $l>l$ auch in den Dialekten von Sernur und Curajeva $i$ und nicht $\hat{\partial}$ antreffen? Vielleicht hat sich das ${ }^{*}$ ? im Anlaut in einer Sonderstellung befunden; man beachte jedoch die lautliche Dop- 
pelheit im BC'-Dialekt: ile $m \sim \hat{\partial} l \hat{z} z ̌ a \cdot m$ 'Feuer fangen; sich erholen'. Das $\hat{a}$ in dem letztgenannten Wort $(\sim \mathrm{W}$ ala $\bullet \ddot{a} m$. $O$ [U] $i l z z a \cdot m)$, das eine Ableitung von dem Verb ile $m$ ist. entspricht in BC der Erwartung. - Es ist wichtig, festzustellen, dass in den $\mathrm{C}^{-}$und C-Mundarten, wo man noch deutliche Spuren des urtscher. ${ }^{*}$ ? wahrnehmen kann, die Entmouillierung des $l$ offenbar erst spät angefangen hat, denn hier hört man noch nebeneinander die verschiedenen Stufen der Entwicklung, $l, l$ und $l$.

Solche Dialekte wie die Mundart von Kiljmez im Kreis Malmyž (M), die Wichus erforscht hat, sowie die von BEKE erforschten Mundarten von Karmankina in Malmyž (MK) und von Petrušin in Uržum (UP) zeigen nicht nur die Entwicklung urtscher. ${ }^{*} ?>i$, sondern fast immer auch urtscher. ${ }^{*}{ }_{\partial}>i$. Die Richtung der Ausgleichung ist also hier umgekehrt gewesen wie in den vorhin behandelten Fällen. Diese $i$-Vertretung ist einer der überzeugendsten Beweise dafür, dass im Urtscheremissischen wirklich neben *a auch * anzunehmen ist. Es entspricht völlig der Erwartung, dass $l$ nach $i$ fast immer seine Mouillierung behalten hat; sogar ein solches $l$, das die Anfangskomponente einer Konsonantenverbindung bildet, kann mouilliert auftreten (z.B. II $k i \cdot l^{\prime} m$ o 'gefroren, erfroren'), was wiederum zweifellos eine sekundäre Erscheinung ist.

Wir wiederholen nun kurz die Hauptzüge des soeben Dargestellten, in dem wir, ohme Vollständigkeit anzustreben, nur einen oder zwei Vertreter jedes uns bekannten Dialekttyps nennen.

Im Westtscheremissischen urtscher. ${ }^{*} a$ und ${ }^{*} ?>a$; in $\mathrm{K}$ kommt in bestimmter Konsonantenumgebung sporadisch ein Lautwandel $a>?$ vor.

In den osttscheremissischen Dialekten sehen wir eine mannigfaltige Entwicklung:

Jaransk-Uržum: Upša ${ }^{*}{ }_{\partial},{ }^{*} ?>\partial$.

Jaransk-Uržum: Turšomučakš *a, *? > $*^{*}$. Ebenso (arevokokšajsk: Marij Ušem.

Carevokokšajsk: Krasnojar $*_{\partial}>\hat{\imath}$, vor $l$ anscheinend auch 
${ }^{*}{ }_{\partial}>$ ?. ${ }^{*}$ ? oft $\equiv$, daneben treffen wir vermutlich sporadisch die Entwicklung $*_{\imath}>\hat{\imath}$ an.

Uržum: Sernur $*_{\partial}>\hat{\partial} .{ }^{*} ?>i$ erstens in geschlossener Silbe ausser vor $l\left(<^{*} \gamma\right)$, zweitens in offener Silbe im Anlaut oder nach einem mouillierten Konsonanten; sonst ${ }^{*} ?>\hat{a}$. Ebenso Birsk: Čurajeva.

Birsk: C̆askino $*_{\partial}>\hat{\partial}$. ${ }^{*} \imath>i$ ausser vor $l\left(<{ }^{*}\right)$, wo gewöhnlich $*_{\imath}>\hat{\partial}$. Den gleichen Dialekttyp vertreten dürfte auch Malmyž: Starinonenger.

Malmy̌̌: Kiljmez, Karmankina ${ }^{*}{ }_{\partial}{ }^{*} \imath>i$ mit ganz wenigen Ausnahmen. Denselben Typ vertritt Uržum: Petrušin. -

Insgesamt ist das urtscher. Phonem $*_{\partial} \sim *^{*}$ in etwa 80 gemeintscheremissischen Wörtern enthalten, von denen Beкe Cs. Ny. 19-22 einen erheblichen Teil aufzählt. Ausserdem kommt es in etwa 20 tschuwassischen Lehnwörtern vor.

Die Entwicklung dieses Lautes auf vortscheremissischer Trundlage bietet folgendes Bild:

a) Urtscher. ${ }^{*} a \sim{ }^{*} ?<$ vortscher. ${ }^{*} e: a \cdot l_{x} m ; i l e \cdot m$, âle $\cdot m$

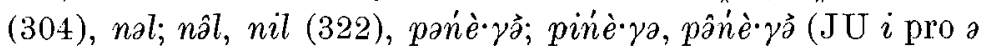
anscheinend unter dem Einfluss des Wortes $p i$ 'Hund', vgl.

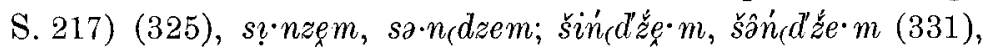
pa·laš; pâlâš, pilâš (344). In diesen handelt es sich um urtscher. *?. - ə•štem; âšte $\cdot m$ (303), lo •štäš; lâšta $\cdot$ š, lištaš (313), šalaž; šâllâz, šìlla (333), kaldem; lâldem, kildem (347). In diesen handelt es sich um urtscher. *a. Unklar ist šiši $m$, šâsâm (332). An Hand der etymologischen Entsprechungen dieses Wortes müsste man eine urtscher. Grundform annehmen, in der sowohl im Anlaut als auch im Inlaut ${ }^{*} s$ gestanden hat. Aber aus der Form *'s? $\cdot$ śam müsste anstelle von P M (BEKE) šâšâm eine Form *šišim entstanden sein. Die heutige Vertretung liesse sich am besten aus einer auf Dissimilation beruhenden Form *so šam erklären. Also haben wir insgesamt 10 Beispiele.

Ausserdem sei auf die folgenden drei Wörter hingewiesen, in denen vortscher. ${ }^{*} e>$ entweder urtscher. ${ }^{*}$ o oder urtscher. n:

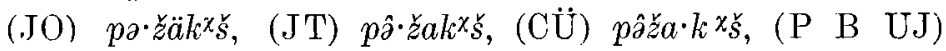




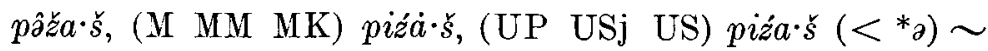

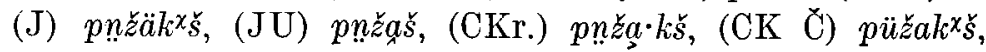
(BJp) püž̋̈ $\breve{s}(<n)(327)$.

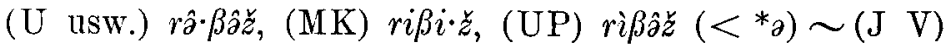

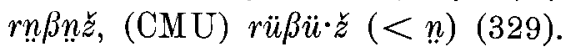

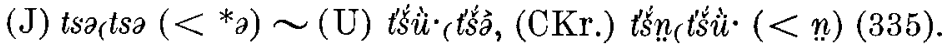
An sich dürfte die Vertretung in den Mundarten kein Kriterium geben, mit dessen Hilfe man entscheiden könnte, ob der Illabial- oder der Labialvokal den älteren Zustand repräsentiert. (In dem letzten Beispielwort allerdings ist die $*_{a}-V e r-$ tretung viel seltener als die n-Vertretung, sie kommt wohl nur in eïnem Dialekt vor.) Aus etymologischen Gründen erscheint $*_{\partial}$ als ursprünglich und $n$ als eine durch sporadische Labialisierung daraus entstandene Nebenform, mit um so mehr Grund, als in allen drei Wörtern die umgebenden Konsonanten einer Labialisierungstendenz günstig sind.

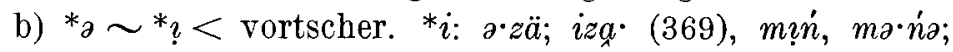

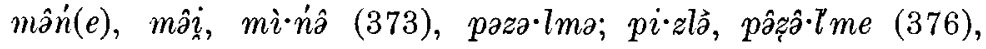
pal; pâl, pī (379), ? pâštệ.m ( W pi štem) (382; vgl. auch

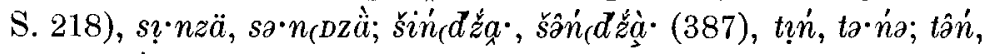
tâ·jà, tiń (388), ša šta; š $\cdot$ štà, šâšte (399), šal; šâl, šil (400).

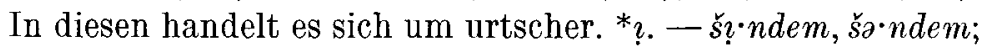
šânde $m$, ṣ̌̂ndem (397), kâre $\cdot m$, liiręr.m (398). In diesen handelt es sich um urtscher. ${ }^{*}$. Insgesamt haben wir $10-11$ Beispiele.

Im Lichte des augenblicklich zur Verfügung stehenden Materials scheint es, dass vortscher. ${ }^{*} e$ und ${ }^{*} i$ im Urtscheremissischen in den meisten Fällen völlig zusammengefallen sind. Die umgebenden Konsonanten bestimmten, ob sich aus ihnen $*_{\partial}$ oder $*_{\imath}$ entwickelte; daneben dürften die analogischen Ausgleichungen -al- $>-\imath l$ - und $-a n->-\imath n^{\prime}$ - von Bedeutung gewesen sein. Eine endgültige Antwort auf unsere Frage können wir jedoch erst dann erhalten, wenn die Forschung auf einem Material aufbauen kann, das sämtliche Dialekttypen umfasst und zugleich die Möglichkeit zu einer genauen Untersuchung der Entstehungsgeschichte und Chronologie der Mouillierung der auf den Vokal folgenden Konsonanten (namentlich $l$ und $n$ ) bietet. Es ist nämlich glaubhaft, dass die 
Klärung der Verhältnisse zwischen den mouillierten und nichtmouillierten inlautenden Konsonanten zusätzliches Licht in einige Einzelheiten auch der Vokalgeschichte bringen wird.

c) ${ }^{*}{ }_{a} \sim{ }^{*} ?<$ vortscher. ${ }^{*} \ddot{u}:$ ka ner; kâne•r, kine·r (415),

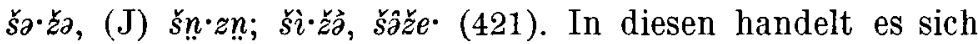
um urtscher. *?. In dem zweiten Wort dürfte J n in Anbetracht seines geringen Verbreitungsgebiets sekundär sein, mit anderen Worten, Ergebnis eines sporadischen Lautwandels $ə>n$. An und für sich wäre natürlich gerade $n$ die zu erwartende Vertretung von vortscher. * $\ddot{u}$. - ka.lma;

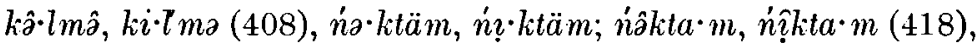

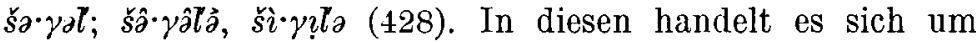
urtscher. *a. Einigermassen unklar hinsichtlich der Vokal-

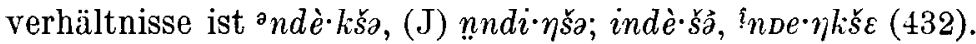
Für die n-Vertretung im J-Dialekt gilt genau dasselbe, was

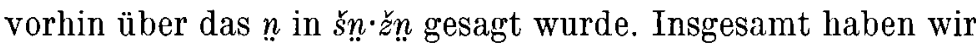
sechs Beispiele.

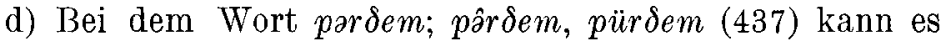
sich um einen unregelmässigen Vokalwechsel $*_{\partial} \sim n$ handeln. Leider ist dieses Wort im Westtscheremissischen nur in der K-Mundart aufgezeichnet worden, die in jedem Fall a hat, einerlei von welchem der beiden Laute man ausgehen muss. Ebensowenig ist klar, auf welchen vortscher. Vokal das urtscher. ${ }^{*}$ bzw. n hier zurückgeht. Die lappische Entsprechung des in Rede stehenden Wortes erlaubt uns, in der Urform ebensowohl $*_{i}$ wie $*_{\ddot{u}}$ anzunehmen.

e) ${ }^{*} a<$ vortscher. ${ }^{*} \ddot{a}: j \imath \cdot l m a, j a \cdot l m a ; j \grave{\partial} \cdot l m a ̀$, nâlme, $j \grave{\imath} \cdot l m \hat{a}$ (287), ? j?p $p^{\varphi} s ; j \partial \hat{p} p \check{s}$ (288). Wenigstens das erste ist ein sicheres $* \ddot{a}$-Wort. In diesen Wörtern ist wahrscheinlich ${ }^{*} j e^{-},{ }^{*}{ }^{*} e^{-}>$

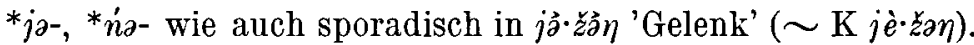
Vgl. S. 211.

f) ${ }^{*} ?<$ vortscher. ${ }^{*} \bar{i}: \beta a t s ; \beta i t^{\prime}, \beta a ̂ t t^{\prime}$ (406). ${ }^{*}$ ? würde an sich besser zur Vertretung von vortscher. ${ }^{*} i$ als von ${ }^{*} \bar{\imath}$ passen. Anderseits aber entspricht der Bestand des Stammkonsonantismus $\left(t^{\prime} s<{ }^{*} t^{\prime}\right)$ der finnisch-lappischen Form *vite und nicht der mordwinischen Form *vitte ( $>$ veite). In diesem Fall wäre die Entwicklung im Tscheremissischen folgende gewesen: *vite $>{ }^{*} \beta$ iit $>{ }^{*} \beta \imath t t^{\prime}>{ }^{*} \beta \imath t t^{\prime}$. 


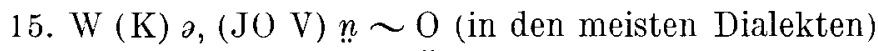
$\ddot{u}$, (JU $\breve{\mathrm{C}} \mathrm{CKr}$.) $n$.

Beispiele hierfür gibt es etwa 70, von denen Beke Cs. Ny. 27-30 etwa 40 erwähnt. Weitere Beispiele sind: $\beta$ ül- 'auf',

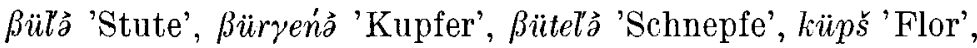
küsedə̉k 'Kiebitz', kü,tšžšta (3. Pers. Sg. Präs.) 'weh tun', lüj̧àsta (3. Pers. Sg. Präs.) 'kitzeln, jucken', pürdem 'zudecken;

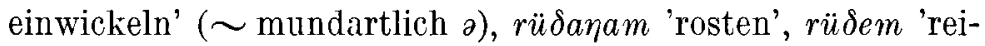

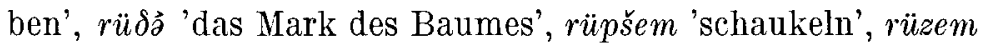
(BČ ruźem!) 'schütteln, rütteln', šüraš 'Gries, Graupe; Grütze, Brei', šüšer (O gewöhnlich šör) 'Milch', šüštả 'weissgegerbtes

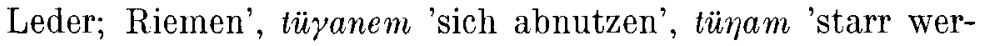
den', tür 'Schneide', tür 'Rand, Ufer', ümàl 'Schatten'. Unter den tschuwassischen Lehnwörtern haben wir wenigstens 20 Beispiele für dies Lautverhältnis.

Im Urtscherenissischen hat es ein reduziertes $n$ gegeben (vgl. Steinitz Vok.96), das sich im Westtscheremissischen in $\mathrm{J}$ und $\mathrm{V}$, im Osttscheremissischen in $\breve{\mathrm{C}}$ und einigen C-Dialekten erhalten hat. In der westtscheremissischen K-Mundart ist $n>a$, aber im grössten Teil des osttscheremissischen Gebiets $n>\ddot{u}$. Somit ist die Entwicklung ron urtscher. $n$ völlig parallel zu der von urtscher. $₹$ verlaufen, vgl. oben S. 201.

Der Ursprung des urtscher. n ist recht einheitlich:

a) $n<$ vortscher. ${ }^{*} \ddot{u}: k a t \check{s}$, liüt'ś (V JU sporadisch $n>\ddot{u}$ ) (409), $k n \cdot n^{d} z e m, k u ̈ n_{(D z e} \cdot m$ (JU wieder $\left.n>\ddot{u}\right)(410), \quad s$,

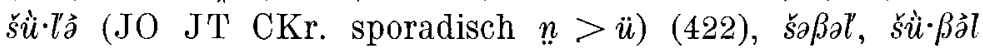

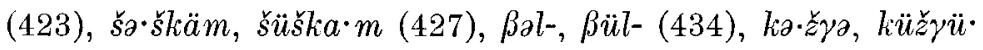
(J V CKr. sporadisch $\ddot{u}$ statt n) (438). Ein wahrscheinlicher ${ }^{*}$ n-Fall, bei dem wir von vortscher. $* \ddot{u}$ ausgehen müssen, ist noch O lü $m \grave{a}$ (429). Bei ša rta, šü rtà (442) geht das urtscher. n?

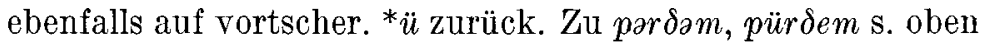
S. 241, Punkt d. Das â in der Form pâr ¿em, die der UJ-Dialekt aufweist, ist eine im Osttscheremissischen alleinstehende Erscheinung. - Beispiele haben wir insgesamt 7-10.

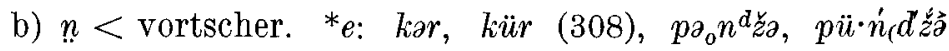

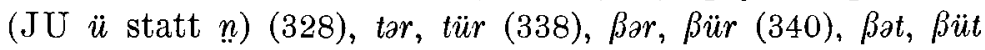


(342). Einen Wechsel $n \sim$ o beweisen die Wörter pâžăš

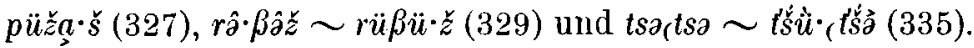
Obwohl sich nicht zwingend nachweisen lässt, welcher von beiden Vokalen im Urtscheremissischen der ursprüngliche war, sprechen Wahrscheinlichkeitsgründe für *a. Vgl. S. 240. - Fälle, die eine urtscher. Entwicklung $n<{ }^{*} e$ zeigen, gibt es somit 5-8. Es besteht gar kein Zweifel darüber, dass es sich in ihnen allen um sporadische, durch die umgebenden Konsonanten verursachte Labialisierung handelt. Darüber näher S. $24 i$, Punkt b.

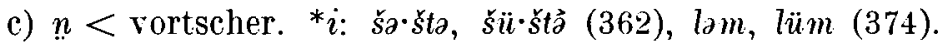
Auch hier handelt es sich um Labialisierung, die durch die umgebenden Konsonanten bedingt ist. Mit der Artikulation des $\breve{s}$ ist ebenso wie mit der der Labialkonsonanten eine Rundung der Lippen verbunden.

d) $n<$ vortscher. ${ }^{*} u$ : tə・rßa, $t \ddot{u} \cdot r \beta \vec{\partial}(209)$. Ein merkwürdiyer Fall von Palatalisierung.

16. Abweichende Lautverhältnisse.

Im Vorstehenden haben wir die Vertretung von 13 urtscher. Vokalen in den heutigen Dialekten und ausserdem zwei Fälle ( W $o, u \sim \mathrm{O} u ; \mathrm{W} i \sim \mathrm{O} e, i$ ) betrachtet, die eine nach der urtscheremissischen Zeit eingetretene, sporadische Differenzierung widerspiegeln. Für die Behandlung dieser beiden Fälle als eigene Gruppe lag kein anderer Grund vor als der, dass auch früher so verfahren worden ist. Grundsätzlich eine Grenze zwischen ihnen und den exzeptionellen interdialektalen Lautverhältnissen zu ziehen, deren Zahl, in Typen gruppiert, nahezu 200 beträgt, fällt recht schwer. Da die Abweichung in der Vokalvertretung eines Wortes sich meist auf eine einzige Mundart beschränkt, haben derartige Fälle keinerlei Bedeutung für die Entstehungsgeschichte des tscheremissischen Vokalismus. Der Gedanke, dass sich in ihnen ein alter finnisch-ugrischer "Ablautwechsel" widerspiegeln könnte, ist schon aus dem Grunde unmöglich, weil solche sporadischen Entwicklungen in gleicher Weise, wenn nicht noch häufiger, auch bei den Lehnwörtern des Tscheremissischen vorkommen. 
Im Folgenden werden Beispiele für diese unregelmässigen Vokalschwankungen gebracht. Wir beschränken uns auf das Material, auf dem unser im Vorstehenden gegebener Überblick über die Entwicklung des tscheremissischen Vokalismus beruht. Somit bleiben viele Schwankungen, die ausserhalb dieses Materials auftreten, ganz unerwähnt.

a) $\mathrm{O} o \sim a$ : porema.m $\sim$ parema.m (34), ßoštar $\sim \beta a s^{-}$

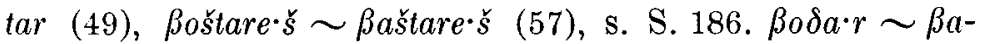
$\delta a \cdot r$ (210), šo $r \delta \hat{\partial} \sim s ̌ a r \delta e \cdot(76)$, s. S. 193. Sehr merkwürdig wirkt U (WICHм.) ka šktâ (98). Es muss sich hier um ein ganz zufälliges Schwanken $o>a$ handeln, denn BEKE hat in drei Mundarten desselben Uržumer Gebiets o aufgezeichnet.

b) $\mathrm{W} \mathrm{O} \approx \sim \mathrm{W}$ o: W O lomem $\sim \mathrm{W}$ (JT K) lo. mem (187). Das in einem engen Gebiet vorkommende $\mathrm{JT} \mathrm{K} o$ ist deutlich < $\rightarrow$. WO $\approx \sim \mathrm{O} o$ : W nolyo, O no.l $\gamma_{0} \sim \mathrm{O}$ nolyo. (226). Über die sporadische Entwicklung $\approx>0$ s. S. $170 .-$ $\mathrm{W} \approx \sim \mathrm{WO} o: \mathrm{W}(\mathrm{K}) m \hat{a} \cdot s ̌ t e m \sim \mathrm{W}(\mathrm{J}) m o \cdot s ̌ t e m, O$ O moštém $(25) ; \mathrm{K} \hat{\partial}<<{ }^{*}$ o.

c) $\mathrm{WO} \approx \sim \mathrm{WO} u$ : W $\beta \circ \cdot r \delta o$, O $\beta \approx \cdot r \delta o \sim \mathrm{W}$ O $\beta u \cdot r \delta \hat{a}$

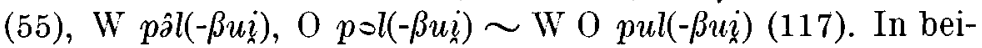
den Fällen $\approx<u$, s. SS. 196, 198. $-0 \approx \sim \mathrm{W}$ O $u$ : 0 p $\bullet \cdot 7 \varsigma s$ $\sim \mathrm{WO}$ pù.lâs (154). Auch hier $\approx \operatorname{klar}<u$. $-\mathrm{W} \approx \sim$ W O $u$ : W (K) pâry- W O pury- (198). Falls dieses tscheremissische Wort nicht ein von den Wörtern der verwandten Sprachen unabhängiger deskriptiv-onomatopoietischer Ausdruck ist, müssen wir sein $\vec{z}$, anders als in den soeben besprochenen Wörtern, als ursprünglich ansehen ( $>$ lautgesetzlich $\mathrm{K} \hat{\partial}, \mathrm{O} u)$, wogegen sich $\mathrm{W} u$ sporadisch daraus entwickelt hätte.

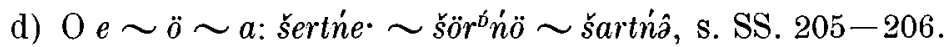

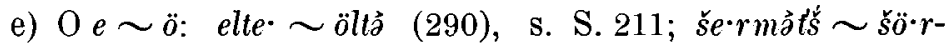
$m n t^{\prime} s(302)$, s. S. 213.

f) $\mathrm{O} i \sim e: i \cdot s k \grave{s} \sim \grave{e} \cdot s ́ k a$ (291). Die zweite Form, die BEKE in der MK-Mundart aufgezeichnet hat, ist ganz vereinzelt. Die Ursache des Lautwandels $i>e$ ist dunkel.

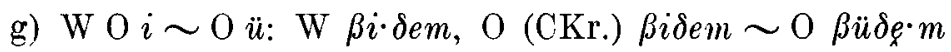
(343), s. S. 217.

h) $0 \ddot{u} \sim \ddot{o}: r \dot{u} \cdot \delta \hat{\partial}, l \dot{u} \cdot \delta \ddot{g} \sim l \grave{o} \cdot \delta \dot{\partial}(384)$, s. S. 222, wo sich 
noch mehr Beispiele finden. Die meisten von ihnen zeigen den Wechsel $\mathrm{W} \ddot{u} \sim \mathrm{O} \ddot{o}$.

i) $0 \ddot{u} \sim i \sim \ddot{o}$ : šün, sün $\sim$ šin $\sim$ šön, sön (163). Die älteste dieser Varianten ist zweifellos $\ddot{u}$, s. Ss. 222, 224.

S. 211.

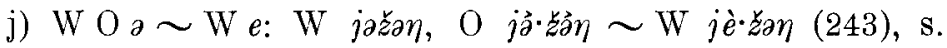

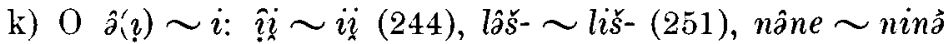

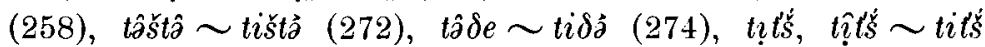
(276). Hier ist sporadisch $i>\partial(\imath)$; s. S. 216 . - W $\sim \sim i$ : pə$z \partial \cdot l m e \sim p i z i \cdot l m a$ (376). Der Vokal der ersten Silbe in der zweiten dieser Formen ist das Ergebnis eines zufälligen Lautwandels $a>i$. - Einen Wechsel $O \hat{a} \sim i$ zeigt das Wort pâza $a m \sim p i \check{z} a \cdot m$ (382), während ein Wechsel $\mathrm{O} *_{a} \sim \mathrm{W} i$ im Wort O pâštę $m \sim \mathrm{W} p i \cdot s ̌ t e m$ auftritt, das aller Wahrscheinlichkeit nach eine Ableitung von dem vorigen ist. S. SS. 181, 218.

l) W O n $\sim$ W O $\ddot{u}:$ W katš, O knțtś $\sim$ W O (JU) küts (409),

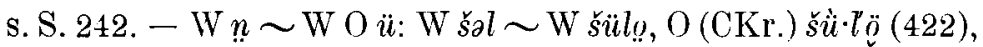

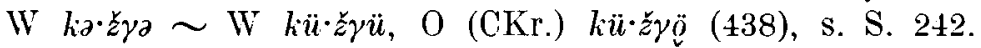
Es ist sehr wahrscheinlich, dass ein Teil der $\ddot{u}$-Fälle in den osttscheremissischen Mundarten lautgesetzlich auf n? zurüchgeht, aber das lässt sich nicht feststellen, weil $n$ und $\ddot{u}$ hier zusammengefallen sind. $-\mathrm{O} n \sim \mathrm{W}$ O $\ddot{u}: \mathrm{O}$ (CKr.) tnz $\sim$ W O tüz (404). Das viel weiter verbreitete $\ddot{u}$ dürfte das ältere

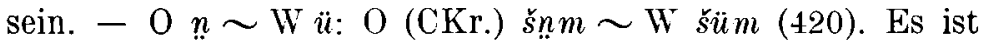
natürlich möglich, dass die osttscheremissischen $\ddot{u}$-Fälle wenigstens teilweise urtscher. $\ddot{u}$ vertreten. Es lässt sich nicht entscheiden, welches von beiden das primäre ist, $n$ oder $\ddot{u}$. $\mathrm{W} n \sim \mathrm{O} \ddot{u}: \mathrm{W} k n \cdot n^{d} z e m \sim \mathrm{O}(\mathrm{JU})$ kündze $\cdot m$ (410). Die osttscheremissische $\ddot{u}$-Vertretung geht auch hier wieder wahrscheinlich - wenigstens teilweise - auf urtscher. n zurück.

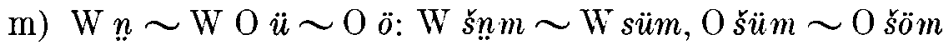
(162). Das $\ddot{u}$ ist primär, s. SS. 222, 224.

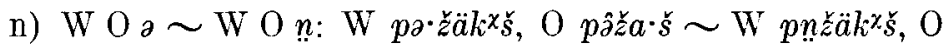

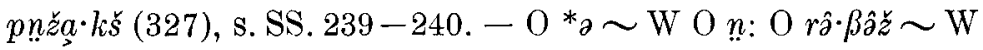

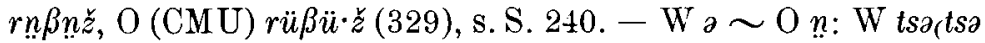

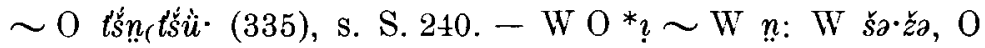

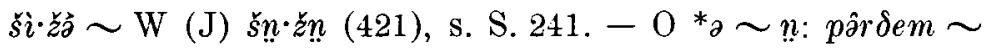


pṇrdem (437), s. SS. 241, 242. - W a n n: ${ }^{a} n d \grave{e} \cdot k$ ša $\sim(J)$ nndi $\eta$ ša (432), s. S. 241.

o) $\mathrm{O} n \sim \mathrm{W}$ O з: O Dieses Lautwechselverhältnis ist insofern von sehr seltener Art, als das eine Glied vorder-, das andere hinterrokalisch ist.

p) Der in der tscheremissischen Sprache selten anzutreffende paradig matische Vokalwechsel, über den Steisitz Vok. 99-101 eine recht erschöpfende Darstellung verfasst hat, ist durchweg sekundär. Oben sind schon alle wichtigeren Fälle eines derartigen Wechsels vorgekommen:

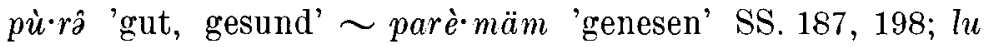
'zehn' $\sim$ laे. $\delta a m$ 'rechnen' S. 201; $\grave{u} \cdot l \hat{a}$ 'es gibt' $\sim \hat{\partial} \cdot l a m$ 'sein' S. 202; $\beta a ̈ r$ 'Stelle, Platz' Berts(an) 'wegen' S. 210; $p i$ 'Hund'

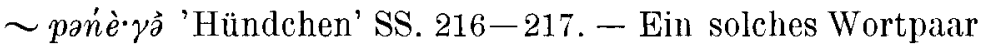

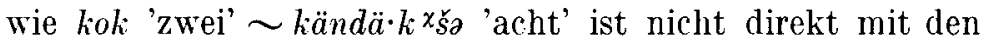
ebenerwähnten zu vergleichen, weil zwischen diesen beiden Wörtern nur ein entferntes Verwandtschaftsverhältnis besteht. Über die Entwicklung des Vokals der ersten Silbe derselben s. SS. $194,205$.

17. Der Einfluss der umgebenden Laute auf die Entwicklung der Vokale.

Im allgemeinen ist der Einfluss der umgebenden Laute auf die Vokale sporadischer Art. Dabei kann man folgende Tendenzen feststellen:

a) Wie Steinitz Vok. 96 beobachtet hat, e r s c h e in e n i m Tscheremissischen Laute, die die reduzierten urtscheremissischen $\approx n$ und * vertreten, nicht im absoluten Auslaut und auch nicht unmittelbar vor einem a n deren Vokal. (Eine Ausnahme von dieser Regel machen nur einige Pronominalstämme.) In den osttscheremissischen Dialekten von Malmyž und Birsk steht statt eines Vordervokals im Auslaut eine auf $-i,-j$ endende Lautverbindung, z.B. kï̈, kü $\sim$ II B $k \ddot{u} i$, BČur. $k \ddot{u} j$ 'Stein'; pi, pi $\sim \mathrm{M} \mathrm{B}$ pii, BČ́ur. pij 'Hund'. Vom Standpunkt der Entwicklung der 
Wortstämme derartiger Struktur betrachtet, wäre meistens der Gedanke natürlich, dass das $i(j)$ trotz seines ziemlich engen Verbreitungsgebiets kein sekundärer Zusatz ist, sondern im Gegenteil auf das Vortscheremissische zurückgeht und einen Konsonanten vertritt, der zwischen der ersten und zweiten Silbe gestanden hat (vgl. oben SS. 217-218). Wenn es sich so verhält, kann man die obige Regel in der Weise erweitern, dass auch vor im Silbenauslaut stehendem urtscher. $i(j)$ statt eines reduzierten Vokals ein Vollvokal erscheint. Von vrrosser Bedeutung für die Untersuchung der Stichhaltigkeit unserer Regel sind die hintervokalischen auf $i$ oder $j$ auslautenden Stämme, weil in ihnen dieser die Silbe schliessende Halbvokal unbedingt schon aus dem Vortscheremissischen stammt. Leider gibt es Beispiele für derartige Wörter alles in allem so spärlich, dass es schwer fällt, zu entscheiden, wieviel Gewicht dem Umstand beizumessen ist, dass die urtscher. Verbindung $*_{-\approx i-}$ - wirklich ebensowenig vorkommt wie die vordervokalischen Lautverbindungen *-niund *-? $i$-. In einem Wort, nämlich $7 u i, l u j$ 'Marder', hat zwar der K-Dialekt $\hat{\partial}$ : lấ, aber da überall sonst im Westtscheremissischen sowie in denjenigen osttscheremissischen Mundarten, wo urtscher. $\vec{z} \equiv$, in diesem Wort $u$ auftritt, so ist es wahrscheinlich, dass in $\mathrm{K}$ eine späte, sporadische Entwicklung $u>\approx>\hat{\partial}$ stattgefunden hat.

Es besteht die Möglichkeit, dass es in einem Teil der Fälle (z.B. lu 'zehn', lu 'Knochen') niemals einen reduzierten Vokal gegeben hat, sondern im Frühurtscheremissischen einen langen Vokal (vgl. S. 197), während in anderen zu sehen ist, dass dem Vollvokal ein reduzierter Vokal rorausgegangen ist (z.B. pii $<^{*} p ?$ ? $\dot{n}$, s. S. 217 ).

Beispiele sind folgende:

$u$ statt des zu erwartenden $s:$ šua.r (170), lu 'zehn' (185), lu 'Knochen' (188), šu (204), ̀̀ (215). S. S. 197. - ü statt des zu erwartenden n: küi (371), püi (378), šüi (426), s. SS. 223, 225. - $i$ statt des zu erwartenden ?: miem (319), pii (325), ii (366), s. SS. $216-217,218$.

b) L a b i a l i s i e r u n g f ä l l e bilden die beträchtlichste Gruppe der sporadischen Vokalentwicklungen im 
Tscheremissischen. Die Ursachen der Labialisierung können folgende Laute sein:

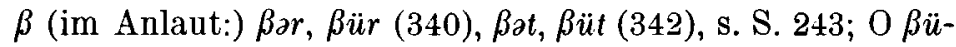
$\delta e^{\prime} m$ ( W $\beta i \delta e m$ ) (343), s. S. 217; (im Inlaut:) $r n \underline{n n z}$, rü $\beta \ddot{u} \cdot \underline{z}$

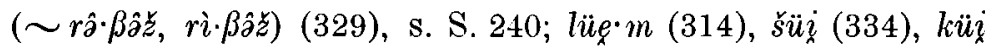
(371), s. S. 223 ; möglicherweise auch $\dot{\partial} \cdot \beta \hat{\partial}(6)$, s. S. 194.

$m$ (im Anlaut:) $m \ddot{u} k \times x_{s}(317), ? m \ddot{u} i(320)$, s. S. 223; möglicherweise ausserdem moštę $m$ (25), mok $\times s ̌ s(27)$, s. S. 194; (im Inlaut:) lam, lüm (374), s. S. 243.

p (im Anlaut:) pört 'Haus', s. S. 214; pük $\chi_{S}$ (259), s. SS.

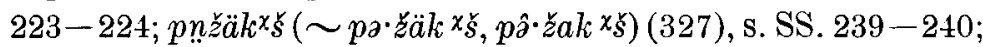
$p \partial_{0} n^{d} z \partial, p \ddot{u} \cdot \dot{n} d \ddot{z} \partial$ (328), s. S. $243 ; p \ddot{u} i$ (378), s. S. 223; man

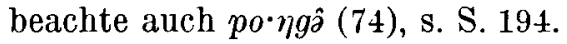

$\check{s}$ (țș̆) (im Anlaut:) šüi (334; vgl. auch oben die $\beta$-Fälle); (im Inlaut:) mošte $\cdot m$ (25; vgl. auch oben die $m$-Fälle); to šta (79);

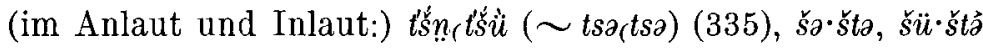
(362), s. S. 243.

$r$ (im Anlaut:) rǜ $\delta \hat{a}$ (384), s. S. 223; rün (407), s. S. 225; (im Inlaut:) kar, kür (308), tər, tür (338), s. SS. 242-243.

Alle diese Labialisierungsfälle sind sporadisch. Man kann über 130 gemeintscheremissische Wörter aufzählen, in denen die obigen Konsonanten im Anlaut stehen und doch nicht die Labialisierung des folgenden Vokals bewirkt haben.

c) Die $\mathrm{P}$ a la t a lis i e r u ng eines ursprünglichen $\mathrm{H}$ i nt e r v o k a ls haben folgende Konsonanten verursacht:

$j$ (im Anlaut:) jükš (91), s. S. 223; jüam (231), s. S. 225. (Für Palatalisierung, die durch $j$ nach der urtscheremissischen Zeit bewirkt worden ist, gibt es recht reichlich Beispiele, von denen hier nur zwei erwähnt seien: O jörem 'umrühren' [ W jarem], O jülem 'brennen' [ W jâlem].) (Im Inlaut:) mör (28), nồ rả

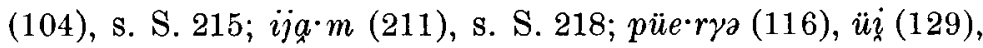
s. S. 223.

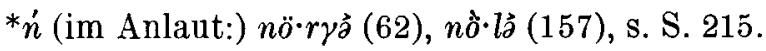

*ś (im Anlaut:) šù $\delta \partial \grave{~(43), ~ s u ̈ m, ~ s ̌ u ̈ m ~(162), ~ s . ~ S . ~} 224$.

Auch diese Lautentwicklungen sind alle sporadisch.

d) $\mathrm{S}$ o h l i e s s u n g von Vokalen durch Einwirkung der darauffolgenden Konsonanten:

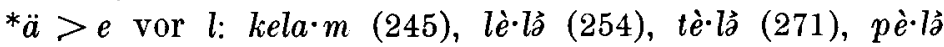


(282), mel (285), elte (290). S. S. 211. Diesen Lautwandel können wir als regelmässig betrachten.

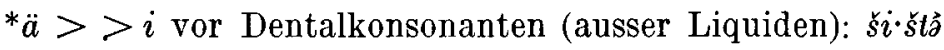
(240), kit (249), liš- (251), nì nà (258), pi š́tà (260), tì štà (272),

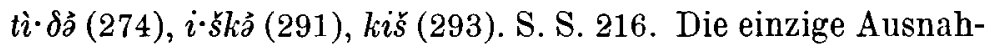
me von der Regel dürfte $k \grave{a} \cdot \delta \partial, k \grave{e} \cdot \delta e$ 'Taube' sein, s. S. 210.

Frühurtscher. ${ }^{*} \partial>{ }^{*} \imath$ vor einem mouillierten Konsonanten, s. SS. 226-227 (Beispiele), 231.

Der Wandel von Vordervokalen zu $i$ vor $i(j)$ zeigt sich regelmässig dann, wenn das $i(j)$ zu derselben Silbe gehört hat wie der ihm vorausgehende Vokal. ${ }^{*} \ddot{a}>i$ : ii (244), ši

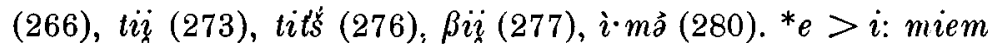
(319), pii (325). S. SS. 216-217.

Die Schliessung von Hintervokalen vor mit $k$ beginnenden Konsonantenverbindungen kann man wohl als eine häufige, wenn auch sporadische Tendenz ansehen. Früh-

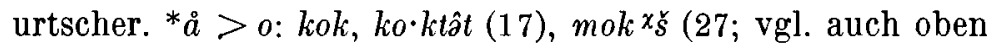

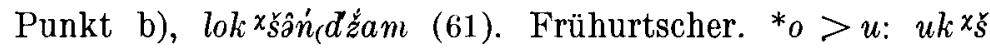

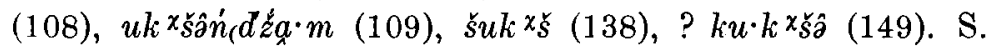
SS. 194, 196-197.

e) Offe $n$ werde $n$ von Vokalen durch Einwirkung der darauffolgenden Konsonanten.

Vor allem kommt hier die Einwirkung von $r$ in Frage. Das $\ddot{o}$ der tscheremissischen Sprache ist ursprünglich infolge der Lautentwicklung -ür- $>-\ddot{o r}$ - entstanden, s. S. 214. Im Osttscheremissischen hat nach der Erklärung, die oben auf S. 220 für den Wechsel $W i \sim O e$ gegeben worden ist, ein sporadischer Lautwandel $i>e$ stattgefunden, wozu anscheinend auch der auf den Vokal folgende Konsonant beigetragen hat. Eine derartige Wirkung hat auch hier vor allem das $r$ ausgeübt. -

Man könnte noch weitere Gesichtspunkte betreffend den Einfluss der umgebenden Laute auf die Vokale der ersten Silbe im Tscheremissischen vorbringen, aber da deren Behandlung wenigstens bei dem jetzigen Stand der Forschung einen recht hypothetischen Charakter tragen würde, lassen wir sie auf sich beruhen. 
18. Die Behandlung der einzelnen vortscheremissischen Vokale im Urtscheremissischen.

$$
{ }^{*} a \text {. }
$$

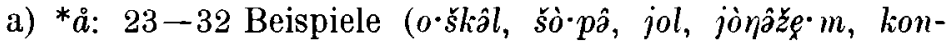

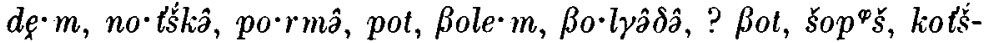

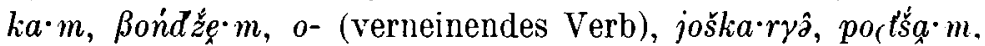

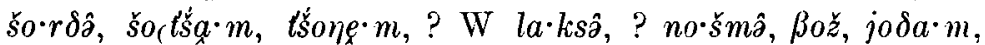

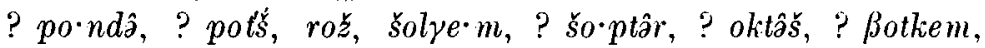
? mostem, 'müde werden', s. SS. 190, 194). Dieser Laut ist die lautgesetzliche Vertretung des ursprünglichen * $a$.

b) o: 12-15 Beispiele $(\grave{o} \cdot \beta \hat{a}, k o \delta \ell \cdot m, k o k, k o l$, mošte $\cdot m$ 'köı-

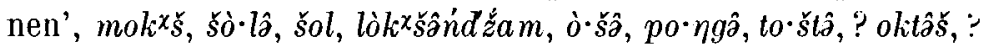
Botkem, ? moštem, 'müde werden', s. SS. 193-194). Die Entwicklug ist ${ }^{*} a>{ }^{*} a>0$ gewesen. Die sporadische Schliessun $r$ des $*^{*} a$ ist hauptsächlich in solchen Wörtern vor sich gegangen. in denen die umgebenden Konsonanten diesen Lautwande] gefördert haben.

Die übrigen Vertretungsarten sind vereinzelte Fälle mit geringer Frequenz:

c) $u$ : 4 Beispiele $(n \grave{u} \cdot \delta \hat{\partial}, p \grave{u} \cdot r \hat{\partial}, \beta u \cdot r \delta \hat{\partial}, k \grave{u} \cdot z \hat{z} k$, s. S. 198). Diese Vertretung bedeutet grundsätzlich eine sporadische Vermischung der ursprünglichen ${ }^{*} a$ - und ${ }^{*} o$-Reihen.

d) $\ddot{o}$ : 2 Beispiele (mör, nö·ryà, s. S. 215). Hier handelt es sich um eine Art Untervariante der in Punkt b) erwähnten Vertretung, denn die Entwicklung ist ${ }^{*} a>{ }^{*} a>^{*} o$ gewesen, wonach das $*_{o}$ dann, wenn ein $j$ oder $\dot{n}$ in dem betreffenden Wort gestanden hat, durch dessen Einfluss palatalisiert worden ist.

e) $\ddot{u}: 2$ Beispiele ( $\ddot{u} l, s ̌ u ̈ \cdot \delta \partial े, ~ s . ~ S S . ~ 224-225)$. Ein Erklärungsversuch für beide Fälle ist a.a.O. gegeben.

$$
\text { *o. }
$$

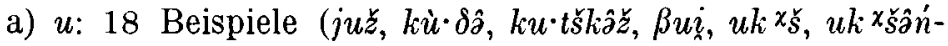

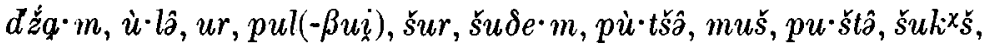
$k u \cdot k^{\chi} \hat{s} a \hat{a}, k \grave{u} \cdot z ̌ a ̂, p \grave{u} \cdot l a ̂ s$, s. S. 196).

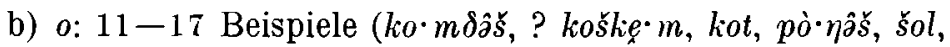




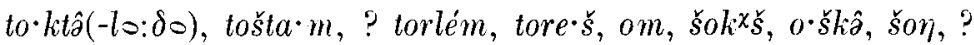

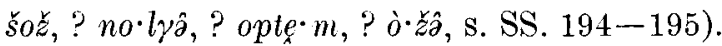

Die Teilung des $*_{o}$ in urtscher. $u$ und $o$ hat sich so gleichmässig vollzogen, dass man beide Vokale als normale Fortsetzer des $*_{o}$ ansehen muss. An und für sich vertritt das o natürlich ein älteres Entwicklungsstadium, von dem man durch eine sporadische, wenn auch sehr häufige Schliessung zu $u$ gelangt ist. Offenbar haben die umgebenden Konsonanten in vielen Wörtern den Lautwandel $o>u$ gefördert.

Vereinzelte Fälle:

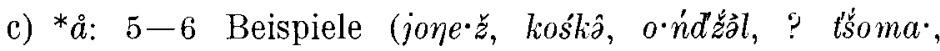
$\check{s}^{\circ} \cdot n d \hat{a}$, ßož, s. S. 190). Hier handelt es sich um einen zufälligen U்bergang aus der ${ }^{*}{ }_{0}$-Reihe in die ${ }^{*} \dot{a}$-Reihe, eine Parallelerscheinung des Lautwandels ${ }^{*} \dot{a}>0$ und $o>u$, aber in umgekehrter Richtung.

d) 3 : 3 Beispiele $(\beta u(t) s \cdot m, u l a \cdot m$ [ urtscher. $u$ in $\grave{u} \cdot l \hat{\jmath}]$, $u z$ ale $\cdot m$ ). S. S. 202, wo die Wörter, die diese aussergewöhnliche Lautvertretung aufweisen, näher betrachtet sind.

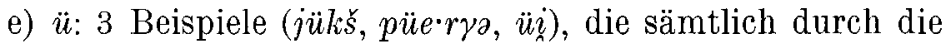
umgebenden Konsonanten veranlasste sporadische Palatalisierungsfälle sind, s. S. 223.

f) $\ddot{o}$ : ein einziger durch inlautendes $*_{j}$ veranlasster Palatalisierungsfall: $n \hat{o} \cdot r \grave{a}$. S. S. 215.

g) $i$ : ein einziges unsicheres Beispiel $(i j a \cdot m)$, das ein auf der Einwirkung eines dem Vokal folgenden $i(j)$ beruhender Palatalisierungsfall ist, s. S. 218.

a) o: 2-4 Beispiele (kolex $m$, ? kož, lo $m b \hat{a}, ?$ kon, s. S. 195).

b) ü: 2 Beispiele (süm, šün), von denen das eine den palatalisierenden Einfluss des im Anlaut gewesenen * $s$ widerspiegelt, während der vordervokalische Charakter des zweiten unerklärt bleibt; s. S. 224.

c) $\ddot{o}$ : ein Beispiel ( $n \grave{o} \cdot l a \vec{a}$, bei dem die Einwirkung des anlautenden ${ }^{*} \dot{n}$ massgebend gewesen ist, s. S. 215. Da die Palatalisierung während der Periode vor sich gegangen ist, 
in der das Wort ein *o enthielt, kann man diese Vertretung als eine Variante der oben unter a) erwähnten betrachten.

d) $u$ : ein einziges etwas unsicheres Beispiel $(t \grave{u} \cdot m \hat{\jmath} s ̌)$, s. S. 197.

e) *å: ein einziges unsicheres Beispiel (šoi-), s. S. 191.

Die Entwicklung des $*_{\bar{o}}$ ist wie die aller anderen selteneren Vokale uneinheitlich gewesen. Trotz der geringen Zahl der Beispiele können wir wohl doch urtscher. $o$ als die primäre Fortsetzung dieses Lautes ansehen.

$* u$.

a) s: 32-33 Beispiele $(j \grave{u} \cdot m \hat{\partial}, k u-, k u \cdot t k \hat{\partial}, k \grave{u} \cdot m \hat{\partial} k, k \grave{u} \cdot \beta \hat{\partial} l$, $k u z a \cdot m, l u \delta a \cdot m$ [ urtscher. $u$ in lu], lum, lume.m, ? muš-

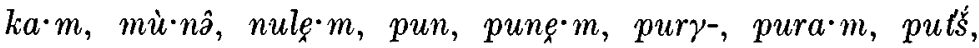

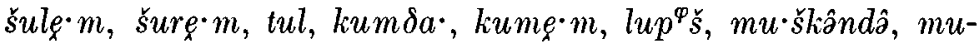

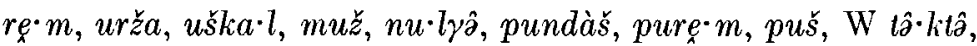
s. SS. 201-202). Die Weiterentwicklung des ursprünglichen ${ }^{*} u$ ist im Tscheremissischen von idealer Regelmässigkeit gewesen. Neben der $\approx$-Vertretung bilden die übrigen hinsichtlich ihrer Frequenz nur unbedeutende Minderheiten:

b) $u$ : 5 Beispiele, (lu 'zehn', lu 'Knochen', š $u$, 'šua·r, $\dot{u}$, s. S. 197). Dies sind Sonderfälle, in denen ausschliesslich ein Vollvokal möglich ist. (Die Wörter sind entweder einsilbige Vokalstämme oder enthalten zwei unmittelbar nebeneinanderstehende Vokale.) Man kann sie also nicht als Ausnahmen von dem Lautgesetz, dass ${ }^{*} u>$ urtscher. $z$, erklären, um so weniger, als in ihnen allen das $u$ ein Ergebnis der Verschmelzung von $* u$ und dem darauffolgenden Konsonanten sein kann.

c) $0: 2$ etwas unsichere Beispiele (tola $\cdot m$, ton), s. S. 195 .

d) $* \stackrel{\circ}{a}: 1-2$ Beispiele $(\beta o \delta a \cdot r$, ? koja $\cdot)$, s. S. 191.

e) n: ein Beispiel (tü.r $\beta \vec{z})$, s. S. 243.

f) $i$ : möglicherweise ein Beispiel $(i j a \cdot m)$, in dem der Vordervokal auf der palatalisierenden Wirkung des darauffolgenden $i(j)$ beruht, s. S. 218. 
${ }^{*} \bar{u}$.

a) $u$ : 3 Beispiele (kut, $p u, r s u e \cdot m$ ), von denen jedoch das zweite und dritte als Sonderfälle anzusehen sind und somit keine volle Beweiskraft haben. S. S. 197.

b) $o: 1-3$ Beispiele $(k o l a \cdot m$, ? kon, ? kož), s. S. 195.

$* \ddot{a}$.

a) $i$ : 15-16 Beispiele $(\beta i \ddot{n}, \ddot{i}, i \cdot m \vec{s}, \check{s} i-, t i \ddot{n}, i \cdot s ̌ k s, k i \check{s}, l i \grave{s}-$,

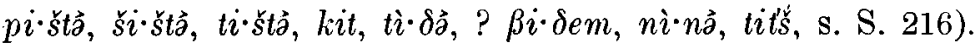
Dieser Lautwandel ist vor $i(j)$ und vor Dentalkonsonanten ausser den Liquidae vor sich gegangen.

b) $e$ : 10-11 Beispiele $(j \grave{e} \cdot z \partial \partial, k e l a \cdot m, k e \cdot r \gamma \vec{a}, k e r t a \cdot m, l \grave{e} \cdot l \grave{a}$, šere $\eta g \grave{\partial}, t \grave{e} \cdot l \grave{a}, p \grave{e} \cdot l a, m e l, k e m$, elte $\cdot$, s. S. 211); in fast allen folgt auf den Vokal die Liquida $l$ oder $r$.

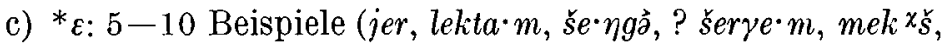
$\beta e \cdot r \gamma \grave{\partial}$, ? leveš, ? šer, ? me, ? te, s. S. 209); in ihnen folgt auf den Vokal $r, \eta$ oder $k$, nur in zwei unsicheren Fällen handelt es sich um einen einsilbigen Vokalstamm.

Vereinzelte Fälle:

d) $\ddot{u}$ : 2 Beispiele $\left(j \ddot{u} k \chi_{s} e_{x} \cdot m, p \ddot{u} k \chi_{s}\right)$, die auf SS. 223-224 betrachtet worden sind.

e) *a: $1-2$ Beispiele $(j \vec{\partial} \cdot \operatorname{lm} \vec{\partial}, ? j a ̂ p \breve{s})$, in denen die Reduktion des Vokals anscheinend eine Folge der sporadischen Einwirkung des anlautenden $j$ bzw. ${ }^{*} n$ ist. S. S. 214.

Die Geschichte des * $\ddot{a}$ zeigt anschaulich, einen wie bedeutungsvollen Anteil die umgebenden Konsonanten an der $\mathrm{He}-$ rausbildung des urtscher. Vokalismus zuweilen hatten.

$$
* e \text {. }
$$

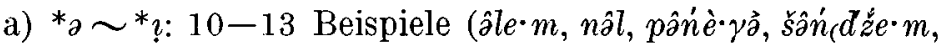

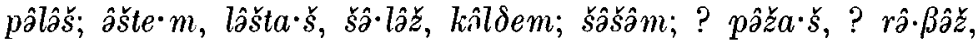
? W (J) $\left.t s a_{(} t s a\right)$, s. SS. $239-240$.

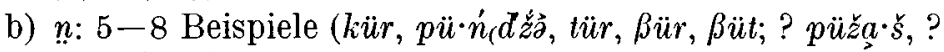

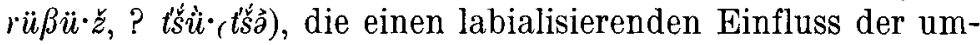
gebenden Konsonanten zeigen, s. SS. 242-243. 


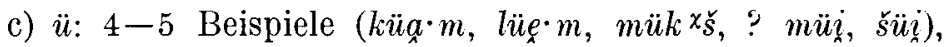
in denen der Grund der Labialisierung meistens der gleiche ist wie in Punkt b), s. S. 223. Vier dieser Wörter haben einen einsilbigen Vokalstamm (bzw. einen auf $i$ auslautenden Stamm) und in dieser Stellung ist nur ein Vollvokal als Sonant der ersten Silbe möglich. Somit kann man hier vom Standpunkt der Lautgeschichte das $\ddot{u}$ durchaus für eine Variante des $n$-Phonems halten.

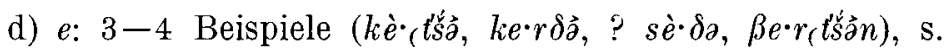
SS. 212-213. In dreien dieser Wörter handelt es sich um einen ursprünglichen zweisilbigen $\ddot{a}$-, $a$-Stamm.

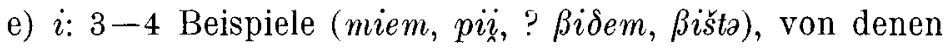
zwei einsilbige Vokal- bzw. Diphthongstämme sind. Somit kann ihr Vollvokal ursprünglich nur eine Variante des Phonems ${ }^{*} \sim{ }^{*}$ ? sein. S. SS. 216-217.

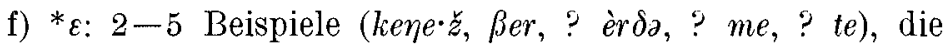
Übergänge in die *ä-Reihe sind; s. S. 209 b) und c).

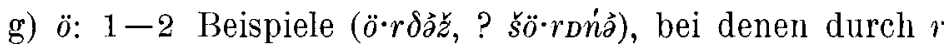
hervorgerufene Labialisierung vorliegt; s. S. 214. Vom Standpunkt der Lautgeschichte können wir die ö-Vertretung hier somit als Variante der in Punkt d) angeführten $e$-Vertretung ansehen.

Wenn, wie oben vermutet, die $i$-Vertretung eigentlich eine Untervariante von ${ }^{*}{ }_{\partial} \sim{ }^{*} ?$ und ebenso die $\ddot{u}$-Vertretung eine Untervariante von $n$ ist, so kann man als die eigentlichen Fortsetzer des vortscher. ${ }^{*} e$ die in den Punkten a) und b) genannten Laute betrachten, während die übrigen Vertretungsweisen vereinzelte Fälle sind, die sich entweder aus diesen entwickelt haben oder auf Reihenübergängen beruhen oder in sonstiger Weise Ausnahmen bilden.

\section{$*_{\bar{e}}$}

a) $e: 4$ Beispiele (lem, nela $m$, pel, ner), s. S. 212.

b) i: 1-2 Beispiele (liam; ? šin šen), s. SS. 217-218, 221. 


\section{$* i$.}

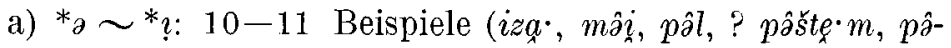

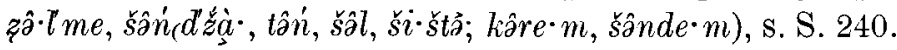

Vereinzelte Fälle:

b) $\ddot{u}$ : 3 Beispiele ( $k \ddot{u} i$, p püi, $r \dot{u} \cdot \delta \hat{z}$ ), von denen zwei einsilbige Vokal- bzw. Diphthongstämme sind und somit wahrscheinlich die vollvokalisch gewordene Variante des in Punkt e) erwähnten $n$-Phonems enthalten. S. S. 223.

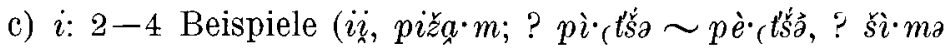
$\sim \breve{s} \grave{e} \cdot m a ̉)$, von denen das eine ein einsilbiger Vokal- bzw. Diphthongstamm ist. S. SS. 218, 220-221.

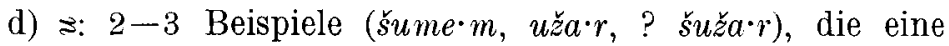
sporadische Velarisierung von $* i$ vor einem $* a$ in der zweiten Silbe widerspiegeln, s. S. 203.

e) n: 2 Beispiele (šüšts, lüm), in denen die umgebenden Konsonanten die Labialisierung veranlasst haben; $\mathbf{s}$. S. 243.

$$
* \bar{\imath}
$$

a) $\ddot{u}: 1-2$ Beispiele (? tü·jnž, rün), s. S. 225 .

b) $i$ : ein Beispiel (nii), das ein einsilbiger Vokal- bzw. Diphthongstamm ist. Diese Vertretung unterscheidet sich also grundsätzlich nicht von der unten in Punkt c) erwähnten Vertretung. S. S. 218.

c) *?: ein Beispiel ( $\left.\beta a \hat{t} t^{\prime}\right)$, dessen Lautvertretung auf $\mathrm{S}$. 241 Punkt f) behandelt ist.

$$
* \ddot{u} \text {. }
$$

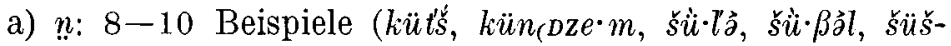

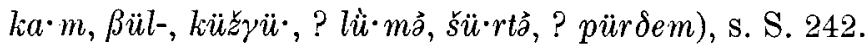

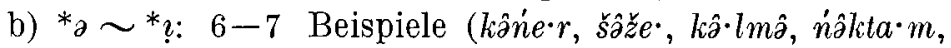

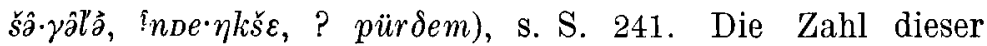
Delabialisierungsfälle ist erstaunlich gross und man kann diese Erscheinung wohl nicht anders erklären als durch die Annahme, dass der im Frühurtscheremissischen sporadisch, 
aber sehr häufig vorgekommene Lautwandel $*^{*} \sim{ }^{*} \imath>n$ den Übergang aus der einen Vokalreihe in die andere auch in umgekehrter Richtung $\left(n>>^{*} a \sim{ }^{*}\right)$ ) erleichtert hat. - Es lässt sich natürlich die Frage aufwerfen, ob das ostseefinnische $\ddot{u}$ gegenüber dem Illabialvokal, der in den tscheremissischen Entsprechungen der betreffenden Wörter erscheint, mit Sicherheit ursprünglich ist. Bei ein paar Wörtern müssen wir diese Frage offen lassen, aber bei den meisten beweist der Lautbestand in verschiedenen fi.-ugr. Sprachen die Ursprünglichkeit des $* \ddot{u}$.

Vereinzelte Fälle:

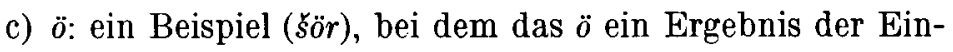
wirkung des auf den Vokal folgenden $r$ ist, s. S. 214.

d) $i$ : ein Beispiel $(i \cdot k t \grave{s})$, s. S. 218.

e) $\ddot{u}$ : ein Beispiel ( $(\tilde{u} \ddot{\imath})$, das ein einsilbiger Vokal-bzw. Diphthongstamm ist. Den Vokal dieses Wortes kann man mit ziemlicher Sicherheit als Variante des n-Phonems ansehen. S. S. 225.

f) $e$ : möglicherweise ein Beispiel (erryả), s. S. 213.

\section{Die Frequenz der Vokale.}

Gemeintscheremissische nichtderivierte Stammwörter, die keine Lehnwörter sind, kennen wir ziemlich genau 1000 . Die Frequenzverhältnisse der Vokale der ersten Silbe sind bei ihnen, prozentual ausgedrückt, etwa folgende: ${ }^{*} a$ und $\approx 12$, $o$ und ${ }^{*} a \sim{ }^{*} ?$ 8, $u$ 7.5, n 7, $i 6$ (wenn man die Fälle von $W i \sim$ $0 e$ abrechnet, 4.5), * $\ddot{a}$, $e$ und $\ddot{u} 5, a$ und ${ }^{*} \varepsilon 3, \ddot{o}$ 2.5. - Die Gesamtfrequenz der unregelmässigen, meist nur in einem einzigen Fall auftretenden Vokalverhältnisse beträgt $16 \%$.

Im Tscheremissischen gibt es über 300 Wörter, die in den verwandten Sprachen Entsprechungen haben, und die urtscheremissische Qualität des Vokals der ersten Silbe lässt sich in 280 Wörtern genau bestimmen. Vergleichshalber zählen wir auch die Frequenzzahlen der Vokale in diesem ältesten Wortmaterial auf: $\approx 14, o$ und ${ }^{*} \dot{a} 13, u 12,{ }^{*} ə \sim{ }^{*} ?$ und $i 10$, $n 9$, $e$ und $\ddot{u} 6,{ }^{*} \varepsilon 4, \ddot{o} 2, * \ddot{a} 1 \%$; a fehlt ganz. 
Die beiden obenangeführten Statistiken lassen sich natürlich nicht ganz gut miteinander vergleichen, weil die Prozentzahlen der ersteren wegen der ziemlich grossen Gruppe der unklaren Verhältnisse durchgehend kleiner sind als die der letzteren. Wir sehen jedoch, dass im grössten Teil der Vokale die Frequenz während der Sonderentwicklung der urtscheremissischen Periode annähernd unverändet blieb. Die Vokale $o, u$ und $i$ kommen im neueren Wortschatz seltener als im ältesten vor, aber das anfangs sehr seltene $\ddot{a}$ ist in demselben Masse verstärkt worden; und $a$, das erst im Späturtscheremissischen entstand, hat sich einen bleibenden, wennschon bescheidenen Platz im Vokalsystem erobert.

Die vortscheremissische Qualität der Vokale kann man im Material von 255 Wörtern bestimmen. Dort ist die Ordnung wie folgt: ${ }^{*} a 17.5,{ }^{*} o$ und ${ }^{*} u 16,{ }^{*} \ddot{a}$ und ${ }^{*} e 13$, $*_{i}$ und $*_{\ddot{u}} 8, *_{\bar{o}}$ und $*_{\bar{e}} 2.5, *_{\bar{u}} 2$ und $*_{\bar{i}} 1.5 \%$. Die Gesamtfrequenz der Hintervokale ist $54 \%$, die der Vordervokale $46 \%$. - Wenn nur die kurzen Vokale berücksichtigt werden, bekommt man folgende Statistik: ${ }^{*} a 19.5,{ }^{*} o$ und ${ }^{*} u 17.5$, $* \ddot{a} 14.5,{ }^{*} e 14, * \ddot{u}$ und $* i 8.5 \%$.

Der Verfasser hat in der Zeitschrift "Virittäjä» von 1948, S. 138 eine Berechnung der Frequenz der kurzen Vokale der ersten Silbe in 375 finnischen Wörtern aufgestellt, die Entsprechungen in anderen fi.-ugr. Sprachen haben. Die Frequenzverhältnisse in diesen Wörtern, die nachweislich zu den ältesten der finnischen Sprache gehören, sind, in Prozenten ausgedrückt, folgende: $a 22, o 17, u$ und $e 15, i 11, \ddot{u}$ und $\ddot{a} 10$.

Zwischen den Frequenzzahlen des finnischen und des rekonstruierten vortscheremissischen Vokalismus herrscht eine sehr weitgehende Übereinstimmung. Der einzige offensichtliche Unterschied ist der, dass die aus * $\ddot{a}$ entstandenen Laute im Tscheremissischen häufiger sind als $\ddot{a}$ im finnischen Material, wogegen ${ }^{*} a$ im tscheremissischen Wortschatz nicht einen so dominierenden Platz einnimmt wie im finnischen. Wodurch diese Inkonsequenzen bedingt sind, wäre noch zu klären. Mit diesen Zahlen verglichen ist die Frequenz der einzelnen Vokale im heutigen Tscheremissisch wesentlich niedriger, was darauf 
zurückzuführen ist, dass die Zahl der Vokale im Tscheremissischen etwa doppelt so gross ist wie die der kurzen Vokale im Vortscheremissischen.

Wenn wir die letztgenannte Tatsache sowie den Umstand, dass auch die ursprünglichen langen Vokale zu der Herausbildung der Vokale des heutigen Tscheremissisch beigetragen haben, in Betracht ziehen, so können wir sagen, dass die Herleitung des tscheremissischen Vokalismus von dem angenommenen vortscheremissischen Vokalismus gerade auch im Lichte der Frequenzverhältnisse auf ungezwungene Weise möglich ist. Beispielsweise ist $*_{a}^{a}$ die lautgesetzliche Fortentwicklung des vortscher. ${ }^{*} a$. Da jedoch das ${ }^{*} a$ im Urtscheremissischen nicht immer auf der ${ }^{*} a$-Stufe stehen blieb, sondern sich recht oft weiter zu $o$ schloss, ist es natürlich, dass das $z$, die praktisch einzige Fortentwicklung des vortscher. ${ }^{*} u$, einen höheren Platz in der Statistik einnimmt als sein Vorgänger im vortscher. System. Wenn man von der Zahl der tscheremissischen $o$ - und $u$-Wörter die Fälle abzieht, in denen diese Iante andere Vokale als vortscher. ${ }^{*} o$ vertreten (d.h. vortscher. ${ }^{*} a,{ }^{*} \bar{o},{ }^{*} \bar{u}$ und ${ }^{*} u$ ), so stimmt die Frequenz der übrigbleibenden Fälle wahrscheinlich mit der des finn. und des vortscher. o gut überein. Eine recht auffallende Ähnlichkeit zwischen den Frequenzverhältnissen des ältesten tscheremissischen Materials und des Vortscheremissischen ist auch der Umstand, dass in beiden Statistiken zuerst die Hintervokale und dann erst die Vordervokale kommen. Ganz kurz sei noch festgestellt, dass auch hinsichtlich der Frequenz der einzelnen Vordervokale der tscheremissische Vokalismus offenbar seinem Vorgänger gut entspricht, wenngleich die Verhältnisse in dieser Beziehung heute wesentlich komplizierter sind als bei den Hintervokalen.

20. Der tscheremissische Vokalismus als phonologisches

System und seine Entwicklung aus dem vortscheremissischen System.

Wir stellen die von uns angenommenen vortscheremissischen und urtscheremissischen Vokalsysteme nebeneinander: 
Vortscheremissisch

$$
\begin{array}{cc}
a & \ddot{a} \\
o-\bar{o} & e-\bar{e} \\
u-\bar{u} & \ddot{u} i-\bar{\imath}
\end{array}
$$

\begin{tabular}{|c|c|c|}
\hline$a$ & & \\
\hline å & & \\
\hline$o$ & $\ddot{\partial}$ & $e$ \\
\hline$u$ & $\ddot{u}$ & $i$ \\
\hline
\end{tabular}

Urtscheremissisch

Im tscheremissischen System sind von den übrigen Vokalen durch eine unterbrochene Linie getrennt die im Späturtscheremissischen entstandenen sekundären Vokale $a$ und ${ }^{*} \ddot{a}$, die für die allgemeine fi.-ugr. Vokalgeschichte nicht von Bedeutung sind. Im unteren Teil des Schemas dagegen sind die Vollrokale und die reduzierten Vokale durch eine Linie voneinander getrennt, weil sie zwei verschiedene Quantitätsreihen vertreten: Erstere treten nach bestimmten Regeln entweder als halblange oder als kurze Laute auf, während letztere (soweit sie sich nicht mundartlich zu Vollvokalen weiterentwickelt haben) stets kurz sind. S. Steinitz Vok. 8-9; Verf., FUF XXIX 245.

Wir können die Betrachtung des tscheremissischen Vokalsystems sehr passend mit den reduzierten Vokalen beginnen. Die drei kurzen geschlossenen Vokale der vortscheremissischen Sprachform entwickelten sich ganz regelmässig zu reduzierten Vokalen (über diesen Vorgang und die Faktoren, die dazu beigetragen haben, s. FUF XXIX 246-255): $* u>z$, ${ }^{*} \ddot{u}>n,{ }^{*} i>{ }^{*} a \sim{ }^{*}$ ?. Mit dem vortscher. ${ }^{*} i$ fiel in den meisten Fällen auch das vortscher. ${ }^{*} e$ zusammen, das also ebenfalls $\mathrm{zu}^{*}{ }^{*} \sim{ }^{*}$ ? reduziert wurde. Die phonetischen Voraussetzungen des Zusammenfallens ron $*_{e}$ und $*_{i}$ sind FUF XXIX 259 - 260 behandelt worden. Auch im Lappischen und Mordwinischen sind $*_{e}$ und $*_{i}$ zum grossen Teil zusammengefallen, eine Entwicklung, die um so verständlicher ist, wenn das $*_{e}$, namentlich vor einem ${ }^{*} e$ in der zweiten Silbe, verhältnismässig geschlossen war, s. unten SS. 278-279.

In den Wörtern aber, die zu einsilbigen Vokal- bzw. Diphthongstämmen wurden, entstanden aus den kurzen Vokalen $*_{u}, *_{\ddot{u}}, *_{i}$ (wenigstens zum Teil über reduzierte Zwischen- 
formen) die Vollvokale $u, \ddot{u}, i$. Vielleicht sind diese geschlossenen Vollvokale ursprünglich gerade auf diese Weise, d.h. als kombinatorische Varianten der reduzierten $\approx$, n und ${ }^{*} \sim^{*}{ }^{*}$, im urtscher. Vokalsystem erschienen. Wenigstens hinsichtlich des $\ddot{u}$ trifft das sicherlich zu. Wenn sich das $u$, $\ddot{u}$ und $i$ jedoch auf die einsilbigen Vokal- bzw. Diphthongstämme beschränkt hätten, wären sie sehr seltene Laute geblieben. Es scheint, dass eine in der Herausbildung begriffene Vokalkategorie, um sich im Vokalsystem einer Sprache erhalten zu können, in ihrer Frequenz eine bestimmte Mindestgrenze überschreiten muss. So dürften diese seltenen $u$, $\ddot{u}$ und $i$ zu Attraktionszentren geworden sein, die vonseiten der ihnen nahestehenden Vokale Zuzug erhielten. Der inerkwürdige Lautwandel $*_{0}>u$, der sporadisch, aber doch oft vorgekommen ist, erklärt sich möglicherweise gerade auf diese Weise, ebenso die Entwicklung $\left({ }^{*} \ddot{a}>{ }^{*} \varepsilon>{ }^{*} e>i\right.$ vor Dentalen mit Ausnahme der Liquidae. Die zahlreichen, ganz unregelmässig wirkenden $\ddot{u}$-Fälle werden gleichfalls verständlicher, wenn man daran denkt, dass das als kombinatorische Variante des $n$ entstandene $\ddot{u}$, um sich erhalten zu können, sein Verbreitungsgebiet im Wortschatz der Sprache unbedingt erweitern musste. Die Zahl der $u$ - und $i$-Fälle erhöhte sich auch durch das vortscher. ${ }^{*} \vec{u}$ und ${ }^{*} \bar{i}$; vorläufig vermögen wir nicht zu entscheiden, in welcher Periode der Quantitätsunterschied zwischen langen und kurzen vortscher. Vokalen sich verwischt hat.

Hinsichtlich der halbengen Vokale kann man annehmen. dass der obenerwähnte Lautwandel $*_{o}>u$ in der Frequenz des $o$ einen Fehlbetrag hervorrief, zu dessen Deckung gleichzeitig mit diesem Lautwandel eine sporadische, aber wieder recht weitverbreitete Lautentwicklung $\left({ }^{*} a>\right)^{*} a ̊ 0$ vor sich ging. Wie das chronologische Verhältnis zwischen dem Lautwandel vortscher. ${ }^{*} \vec{o}>$ urtscher. $o$ einerseits und den Lautentwicklungen $*_{a}>0$ und $*_{o}>u$ anderseits ist, bleibt unentschieden. Es sei jedoch darauf hingewiesen, dass zwischen der Vertretung von vortscher. ${ }^{*} o$ und der von vortscher. ${ }^{*} \bar{o}$ im Tscheremissischen wenigstens kein deutlicher Unterschied besteht, was vielleicht darauf hindeutet, dass der 
Schwund der vortscher. Quantitätsunterschiede eine ältere Erscheinung ist als die obenerwähnten Fälle von sporadischer Schliessung. Das urtscher. $e$, das als Vertreter von vortscher. ${ }^{*} \ddot{a}$ und vortscher. ${ }^{*} \bar{e}$ in solchen Wörtern steht, in denen fast immer $l, r$ oder $m$ auf den Vokal folgt, konnte wohl auf Kosten der Nachbarlaute keinen zusätzlichen Boden mehr gewinnen, weil der einzige dafür in Frage kommende Laut ${ }^{*} \varepsilon$ schon auf seine Minimalfrequenz zusammengeschrumpft war, was man anscheinend auf einen in weitem Umfang vor sich gegangenen spontanen Schliessungsprozess (worüber näher unten) zurückführen muss. Die Entstehung des dritten halbengen Vokals $\ddot{o}$ erscheint nur als Ergebnis einer Art Systemzwang verständlich. Offenbar wurden einerseits $i$ und $e$, anderseits $u$ und $o$ als Pare in vertikaler Linie aufgefasst. und damit das System auch bei $\ddot{u}$ lückenlos wurde, entwickelte sich neben diesem das $\ddot{o}$ durch einen Lautwandel -ür->-ör-. Einen kleinen Zuwachs erhielt das $\ddot{o}$ infolge der Palatalisierungsfälle $o>\ddot{o}$ und anderer seltenen und aussergewöhnlichen Entwicklungen.

${ }^{*} \dot{a}$ und ${ }^{*} \varepsilon$ waren durch eine spontane leichte Schliessung aus vortscher. ${ }^{*} a$ und ${ }^{*} \ddot{a}$ entstanden, und mit Ausnahme des obenerwähnten sporadischen Lautwandels $* \dot{a}>0$ hat sich das ${ }^{*} a$ dann als eigentlicher Fortsetzer des ${ }^{*} a$ erhalten. Hingegen blieb das ${ }^{*} \varepsilon$ nur unter ganz bestimmten Voraussetzungen von der Schliessung verschont, nämlich vor $r$ (jedoch auch dann nicht immer) und vor Palatalkonsonanten. In anderer Stellung schloss es sich auf die oben behandelte Weise zu $e$, sogar noch weiter zu $i$. Seine Frequenz konnte das ${ }^{*} \varepsilon$ dadurch etwas vergrössern, dass offenbar schon früh ein sporadischer Übergang des ${ }^{*} e$ in diese Vokalreihe stattfand (wie die Beispiele beweisen, vor $r$ und $\eta$ ); aber trotzdem blieb die Frequenz des ${ }^{*} \varepsilon$ viel geringer als die des ${ }^{*} \ddot{a}$, seines Vorgängers im vortscher. System.

Als allerletzte entwickelten sich im späturtscheremissischen System die offenen Vokale $a$ und $* \ddot{a}$. Ausser fremdem Einfluss war eine günstige Voraussetzung für ihr Entstehen der Umstand, dass sich vortscher. ${ }^{*} a$ unter bestimmten Bedingungen in anderen als der ersten Silbe unverändert erhalten 
hatte und daneben in dieser Stellung auch die durch die Vokalharmonie veranlasste Variante $\ddot{a}$ auftrat. Mit einigen wenigen Ausnahmen konnten $a$ und $* \ddot{a}$ nicht in das Wortgut eindringen, das in vortscheremissische Zeit zurückreicht, sondern ihr Bereich blieben späte, vorzugsweise deskriptiv-onomatopoietische Wörter sowie die Lehnwörter.

Das tscheremissische Vokalsystem unterscheidet sich sowohl qualitativ als auch quantitativ scharf von dem vortscheremissischen. Seine Herausbildung hat eine völlige Umwälzung des früheren Systems bedeutet. Es kann gar keine Rede davon sein, dass die Weiterentwicklung jedes vortscher. Lautes in allen Einzelfällen mit strenger Regelmässigkeit vor sich gegangen wäre. Die meisten von ihnen werden im Tscheremissischen durch zwei verschiedene Laute vertreten; ausserdem sind Reihenübergänge zwischen qualitativ nahestehenden Lauten eine gewöhnliche Erscheinung. Ungeachtet dieser Tatsachen sind jedoch die allgemeinen Linien der Entwicklung deutlich sichtbar und wenn eine auf den ersten Blick unbegreiflich erscheinende Aufteilung eines vortscheremissischen Lautes vorkommt, so bemerken wir bei tieferem Eindringen, dass sie einem wichtigen Zweck dient: der möglichst geschlossenen und harmonischen Herausbildung des aus den Ruinen des alten sich aufbauenden neuen Vokalsystems. Alle Vordervokale haben eine Serie gebildet, deren Glieder untereinander in enger Verbindung und Wechselwirkung standen, und eine ebensolche Serie für sich bildeten auch die Hintervokale. Wenn eine in der Herausbildung begriffene Vokalkategorie auf dem Wege der "lautgesetzlichen" Entwicklung keine genügend grosse Frequenz erlangen konnte, so boten sporadische Änderungen der nah verwandten Vokale eine Ergänzung; dabei erwies sich die Einwirkung der umgebenden Konsonanten als der Faktor, durch den die erforderlichen Lautänderungen vor sich gingen. Wenn aber wiederum ein solcher Vokal, der durch einen sporadischen Lautwandel einen Teil der Fälle verloren hatte, in denen er auftrat, eine Verstärkung seiner Frequenz brauchte, so erhielt er diese in gleicher Weise von irgendeinem anderen ihm nah verwandten Vokal. So konnte ein einziger Lautwandel eine Reak- 
tion auslösen, die sich fast durch die ganze Kette der Vorderbzw. der Hintervokale fortsetzte. Es konnten sogar Übergänge von Vokalen aus der einen Hauptgruppe in die andere vorkommen, wie besonders die zahlreichen Fälle der Palatalisierung von Hintervokalen beweisen. Auch derartige sporadische und anfangs befremdend erscheinende Veränderungen haben sicherlich ihren Zweck.

Betrachten wir die zuletzt erwähnte Einzelheit etwas näher. Der von uns rekonstruierte vortscheremissische Vokalismus der ersten Silbe enthielt fünf Hinter- und sechs Vorderrokale. Trotz ihrer kleineren Anzahl übertrafen die Hinterrokale hinsichtlich der Frequenz die Vordervokale deutlich: Wie aus den auf S. 25 i angeführten Zahlen hervorgeht, machten die Hintervokale $54^{0}{ }_{i 0}$ aus, die Vordervokale nur $+6^{\circ},{ }_{0}$. Im Frühurtscheremissischen verminderte sich die Anzahl der Hintervokale, aber die der Vordervokale wuchs; an Hintervokalen gab es nun vier, an Vordervokalen sieben (im Späturtscheremissischen waren die entsprechenden Zahlen fünf und acht). Im Lichte dieser Tatsachen verstehen wir vollkommen, dass die Entwicklung der Hintervokale sich in wesentlich regelmässigeren Bahnen vollziehen konnte als die der Vordervokale. Die Vordervokale mussten in ihrem im Wortschatz der Sprache verhältnismässig wenig verbreiteten Liatmaterial ausreichenden Boden für sieben verschiedene Phoneme finden. Es war unumgänglich, einen Ausgleich herbeizuführen, indem die Vokale mit der grössten Frequenz, d.h. vortscher. ${ }^{*} \ddot{a}$ und ${ }^{*} e$, sich in mehrere, zum Teil sehr verschiedene Phoneme teilten und zwar auf eine Weise, die überhaupt zu verstehen schwer fiele, wenn wir nicht annehmen würden, dass Tendenzen, die auf eine Systemganzheit hinzielen, dahinter gestanden und die Entwicklung gelenkt haben. Auch die Fälle von $*_{o}>\ddot{o}$ und die übrigen Palatalisierungsfälle verstehen wir am besten als Ergebnisse des zwingenden Bedürfnisses der Vordervokale, zusätzlich Boden zu gewinnen. Dank diesen ziemlich zahlreichen Palatalisierungsfällen glückte es den Vordervokalen, den Vorsprung um einiges zu vermindern, den die Hintervokale hinsichtlich der Frequenz als Erbe der vortscheremissischen Periode vor ihnen hatten. Die 
Schaffung neuer Wörter während der tscheremissischen Sonderentwicklung hat weiter in der gleichen Richtung gewirkt, denn in der auf S. 256 gebrachten Statistik der Frequenz der Vokale im heutigen Tscheremissisch entfallen auf die Hintervokale $42.5 \%$ und auf die Vordervokale $41.5 \%$. (Die abweichenden Vokalverhältnisse sind hierbei nicht berücksichtigt, weil sie offenbar das allgemeine Bild nicht ändern würden.)

Wie auf SS. 260-261 erwähnt, erscheint es wenigstens im Augenblick nicht als möglich, irgendwelche chronologischen Zwischenphasen in dem Wandel vom vortscheremissischen Vokalsystem zum tscheremissischen zu rekonstruieren, die näheren Aufschluss über den Zerfall des alten Systems, z.B. über die Vermischung der für dasselbe typischen langen und kurzen Vokalquantität geben würden. Es sei jedoch zum Schluss noch festgestellt, dass im Vortscheremissischen ganz offensichtlich eine den ostseefinnischen Lautverhältnissen entsprechende Quantitätsaufteilung geherrscht hat. Das beweisen beispielsweise folgende als normal zu betrachtenden Lautverhältnisse:

Ostseefi. $u \sim$ urtscher. $\approx \quad$ Ostseefi. $e \sim$ urtscher. $*_{\partial} \sim{ }^{*},, n$ " $\bar{u} \sim \quad u, o \quad$ " $\quad \bar{e} \sim \quad e, i$.

DER PERMISCHE VOKALISMUS.

1. Syrj. $a \sim$ wotj. $a$.

Nach Wrchmans, Wotj. Vok. 82, gibt es für dieses Lautverhältnis etwa 100 Beispiele. Diese Zahl erscheint jedoch zı hoch gegriffen. Wenn man die jungen Lehnwörter und die unklaren Zusammenstellungen nicht in Betracht zieht, weist das heute zur Verfügung stehende Material nur etwa 70 syrjänisch-wotjakische $a$-Wörter auf. 
Schon im Urpermischen hat es ganz sicher ein a gegeben. In Anbetracht der ziemlich grossen Zahl von urperm. $a$-Wörtern erweist sich die Zahl der Wörter, für die sich Entsprechungen in den übrigen fi.-ugr. Sprachen finden, als erstaunlich klein. Ausserdem sind auch diese Etymologien in manchen Fällen unsicher.

a) $a<$ vorperm. ${ }^{*} \ddot{a}$. Als die klarsten Fälle erscheinen $n a$ (258), ta- (274), ai (279). Obwohl für das Wort na keine Entsprechung im Wotjakischen bekannt ist, kann man hier auf Grund des Wortes ta- ein urperm. a annehmen. Ein solches haben wir möglicherweise auch in folgenden Wörtern, deren Verbreitung sich auf eine der beiden permischen Sprachen beschränkt: malalni (255), wotj. naani (257), wotj. paš-pu (259), wotj. la ez (298). Die Zurückführung von urperm. a auf vorperm. ${ }^{*} \ddot{a}$ erscheint also in wenigstens $3-7$ Wörtern als möglich.

b) $a<$ vorperm. ${ }^{*} a$. Hierfür gibt es nur zwei Beispiele: bad" (32) und ? vartni (56), von denen wenigstens das zweite sehr unsicher ist. Wenn wotj. vorton: tyl-v. 'Feuerstahl' mit syrj. vartni, vartan zusammengehört, ist es auch bei diesem Wort fraglich, ob es ein urperm. a gehabt hat.

c) $a<$ vorperm. ${ }^{*} e$. Hiervon haben wir zwei unsichere Fälle: mal' (318) und wotj. vaź (339). Im letzteren Wort macht die Qualität des inlautenden Konsonanten die Zusammengehörigkeit mit den aus westlicheren Sprachen beigebrachten Entsprechungen unsicher. Ausserdem bleibt wegen der begrenzten Verbreitung der beiden Wörter in den perm. Sprachen die Frage offen, ob es sich in ihnen wirklich um urperm. $a$ handelt. Auch zarnii (350) könnte in dem Fall zu dieser Gruppe gehören, wenn es unmittelbar mit den entsprechenden Wörtern im Mordwinischen und Tscheremissischen in Verbindung zu bringen ist. Anderseits müssen als Vergleichsobjekte die entsprechenden hintervokalischen Wörter in den ugr. Sprachen in Betracht gezogen werden. Offensichtlich ist diese Edelmetallbezeichnung mehrfach aus verschiedenen arischen Sprachen entlehnt worden.

d) $a<$ vorperm. *ĩ: vaini $(405)$. Könnte man sich die Ent-

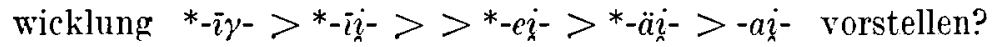


e) $a<$ vorperm. ${ }^{*} 0$. Ein unsicheres Beispiel: kad (101). Da dieses Wort keine Entsprechung im Wotjakischen hat, lässt sich sein urperm. Vokalbestand nicht mit Sicherheit bestimmen und seine Zusammengehörigkeit mit fi. kotra ist auch wegen des inlautenden - $d$ - keineswegs unbestritten.

Es scheint, dass in dem Wortgut fi.-ugr. Herkunft das urperm. $a$ in erster Linie eine Fortsetzung von ursprünglichem * $\ddot{a}$ ist. In keinem Fall jedoch ist es die eigentliche Vertretung des fi.-ugr. *ä in den permischen Sprachen, wie es Steisitz annimmt (z.B. Vok. 39), denn in dem etymologisch als sicherer zu betrachtenden Wortmaterial wird das * $\ddot{a}$ stets durch andere Laute vertreten als durch $a$. Eher ist die Entwicklung $*_{\ddot{a}}>$ urperm. $a$ ein Sonderfall. In einsilbigen Vokalstämmen wie ta und na geht die Entwicklung oft ihre eigenen Wege und etwa ein Wort wie ai kann schliesslich auch ein ron fi. äijä unabhängiges Wort der Kindersprache sein. Die Annahme von Steinitz, dass vor mouillierten Konsonanten und $j$ fi.ugr. ${ }^{*} a>$ urperm. $a$ (Vok. 126), dürfte wohl auf das Wort bad passen, aber alle anderen Wortzusammenstellungen, die er in diesem Zusammenhang vorlegt, sind offensichtlich falsch.

In den arischen Lehnwörtern dürfte urperm. a gewöhnlich einem $a$ in der lehngebenden Sprache entsprechen, z.B. syrj. ameś 'Pflugschar, Pflugmesser', wotj. amedź id., vgl.

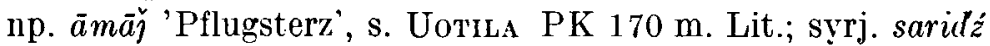
'warme Gegend, wohin die Zugrögel ziehen; Heer', wotj. zariż' 'Meer', vgl. av. zrayah- 'Meer', s. Uotila, a.a.O., S. 39 II. Lit.

Man erhält den Gesamteindruck, dass das urperm. a ein Laut ist, der sich hauptsächlich auf den späten permischen Spezialwortschatz beschränkt, in einigen seltenen Fällen jedoch auf die vorperm. Zeit und dann namentlich auf $* \ddot{a}$ zurückgeht. Ob die Entwicklung den Verlauf ${ }^{*} \ddot{a}>{ }^{*} \dot{a}>a$ genommen hat, lässt sich heute wohl unmöglich mehr sagen. Warum LYткм in einer Tabelle in Древнепермский язык 88 das $a$ der altpermischen Schriftsprache als einen Mittelvokal vermerkt, ist unklar. Phonetisch ist ja sowohl das syrjänische als auch das wotjakische a ein Hintervokal (s. z.B. WicHMANN Wotj. Vok. S. V) und ein solcher muss es bereits im 
Urpermischen gewesen sein, also in einer Sprachform, die etwa 500 Jahre vor der Entstehungszeit der ältesten syrjänischen Sprachdenkmäler ihr Ende gefunden haben dürfte.

Die Geschichte des urperm. a erinnert in gewissem Grad an die des urtscher. a. In der ersten Silbe kommen diese beiden Vokale meist im jüngeren Wortgut vor, aber in den nichtersten Silben hat sich das alte fi.-ugr. $a$ in bestimmten Fällen nicht bloss im Tscheremissischen, sondern wahrscheinlich auch in den permischen Sprachen erhalten. Im Permischen hat das $a$ der ersten Silbe jedoch eine viel grössere Frequenz als im Tscheremissischen und ist nach allem zu schliessen im permischen Vokalsystem verhältnismässig nicht so spät entwickelt worden wie in dem der tscheremissischen Sprache.

\section{Syrj. (auch SO PO) $o \sim$ wotj. $u(u)$.}

Wichmaxi, Bubricin, Steinitz u.a. haben im Syrjänischen nur mit einer einzigen Qualität des o-Vokals operiert. Lyтkix hat jedoch im Jahr 1930 gezeigt (Сорорник компсспг по собиранюю словаря І изучению дналектов коми языка, выпуск I, SS. 32-34), dass im Dialekt der OberSysola (SO) zwei $o$-Vokale vorkommen: ein "gewöhnliches offenes" $o$ und ein geschlossenes $o$, von dem Lytkin sagt, dass Lippen und Zahnspalt dabei in der gleichen Stellung sind wie bei der Artikulation des $u$, die Zungenartikulation aber an derselben Stelle ausgeführt wird wie bei der Artikulation des gewöhnlichen $\boldsymbol{o}$. In der von ARvid GeNeTz erforschten ostpermjakischen Mundart (PO) entspricht, wie Lytkin weiter feststellt, dem geschlossenen $o$ des SO-Dialektes $u$; im Wotjakischen wiederum entspricht sowohl dem gewöhnlichen als auch dem geschlossenen $o$ ein $u$. Auf die Frage der zwei $o$-Vokale in der syrjänischen Sprache kommt Lytkin im Jahr 1952 in seinen Untersuchungen "К вопросу о вокализме пермских языков", SS. 73-80, und "Древнепермский язык", SS. 88-90, zurück. Er bringt dort die interessante Angabe, dass diese Laute in einigen alten syrjänischen Sprachdenkmälern durch verschiedene Buchstaben bezeichnet werden. Es wird somit aus allem ersichtlich, dass die Wurzeln der beiden 0 -Laute 
der SO-Mundart in sehr alte Zeiten zurückreichen. UoTILA hatte während des zweiten Weltkriegs Gelegenheit, die Sprache eines im Dialektgebiet der Ober-Sysola beheimateten Kriegsgefangenen $\mathrm{zu}$ erforschen, und auch er stellte darin zwei $o$-Laute fest, $o$ und $o$ (JSFOu LII 5, S. 7).

In diesem Kapitel behandeln wir nur den gemeinsyrjänischen "gewöhnlichen" $o$-Vokal, als dessen Entsprechung im Wotjakischen $u$ anzutreffen ist. Es dürfte für unsere Untersuchung nützlich sein, alle in Uotilas Aufzeichnungen vorkommenden $o$-Wörter der SO-Mundart aufzuzählen und zugleich ihre Entsprechungen im Ostpermjakischen und im Wotjakischen zu nennen, soweit diese bekannt sind (auch solche Wörter, bei deren wotjakischer Entsprechung ein abweichender Vokal auftritt, sind genannt). Die Wörter ohne Dialektbezeichnung vertreten die S()-Mundart. Ein ähnliches Verzeichnis hat Lytkin in Tруды I, SS. 79-80.

bobul 'Schmetterling', wotj. bubịli; bolett'sini (vain) '(im Wasser) verschütten', PO bótet-; bon 'Lindenbast', PO bon, wotj. bun; bord 'Flügel', PO bord, wotj. burd; PO bóśt- 'nehmen, bekommen', wotj. baśtini; doimini 'sich verletzen', PO dójd-, wotj. dei; doljalni 'unbeschäftigt herumstreichen', Po dóljal-; dolid 'froh, fröhlich', PO dólet; dom 'Zaum', domni. 'binden', PO dom, wotj. dum?̂n̂?; don 'Preis, Wert', PO don, wotj. dun; don 'hell, glühend', wotj. dun; dor 'Rand, Ufer', PO dor, wotj. dur, dor; dorjini 'verteidigen'; dorni 'schmieden', PO dór-, wotj. durị̂n î; dozmer 'Auerhahn', PO dózmor; dzor 'grau', wotj. dźar; dźorjalni 'betrachten'; gogin 'Schabeisen, Schabmesser', PO góget; gol a 'Hals'; gor 'Badestubenofen', PO gor, wotj. gur; gor : t'soi-gor-ulछ 'nach unten an den Abhang', wotj. gureź; gora 'tönend', gorzini 'schreien', PO góra, górzi-, wotj. gur; gorda 'Kriekente', wotj. gurdo; gordźedlini 'rülpsen', wotj. gurdẓ̇̂̂? ; gormeg 'Schürstange'; PO górmog 'Pfeffer'; goř̌ 'Kehle, Gurgel', PO goř̌s; gort 'Haus', PO gort, wotj. gurt; gos 'Fett, Talg', PO gos; PO gótjal- 'gackern'; gožem 'Sommer', PO góžm, wotj. gužem; jodi 'Brachsen'; jog 'Kehricht, Schutt', PO jog, wotj. jug; jokmit 'Bündel'; jon 'stark, kräftig', PO jon, wotj. jun; PO jort 'Kamerad'; PO (Lутк.) koba 'прялка (ручная)'; РО (Lчтк.) kodem 'колодец'; 
kodralni 'für die Verstorbenen beten'; kodźni 'kastrieren', PO kó ${ }^{-}$; kok 'Fuss', PO kok, wotj. kuk; kokalni 'picken', PO kókal-; kokni 'leicht', PO kóknii(t); kolast : kolastin 'zwischen', PO kólas; kolni 'nötig sein', PO kól-, wotj. kulk; kolmini 'von Kohlendunst benommen werden', $\mathrm{PO}$ kólmi-; kom 'Esche'; PO kom 'der hintere Teil im Zimmer'; PO (LYтк.) komet 'промежуток времени, во время чего-нибудь'; komi 'Syrjäne', $\mathrm{PO}$ kómi, wotj. kam; koreg 'Klümpchen'; korgini 'schnarchen'; korni 'bitten', PO kór-, wotj. kurị̂n?; kos 'Zwischenraum', PO kost, wotj. kusị̂p; kosišs 'eine Art Ente'; PO kóšši- 'suchen',

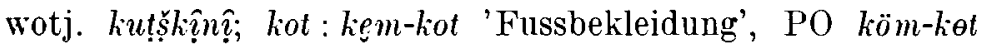
$(o>\theta$ in unbetonter Stellung), wotj. kut; kotertni 'laufen', PO kótert-; kotir : kotiren bịdmg 'es wächst buschig', ? wotj. kot?̣̂ 'um, Umgebung'; koț̣skedni 'klopfen', PO kócki-; kot ț̣sess$d u k$ 'Brandgeruch'; ko f'šg 'Stechen, Stiche', PO kóćag; PO) (Lүтк.) lob- 'головка (лопасть) прялки'; loini 'kneten (Teig, Lehm)', PO lój-, wotj. lajînnị; lokni 'kommen', PO lók(t)-, wotj. lịktîn î; PO lom-pu 'Erle', wotj. lul-pu; loni 'werden, entstehen, geschehen', PO lu< (u!), wotj. luîn lop 'Milz', PO lop, wotj. lup; lop : pań-lop 'Löffelschale'; l'od'ź 'Schwaden'; lok 'böse', PO lok, wotj. lek, leg; lol $\varepsilon$ : il $l_{\varepsilon}-l^{\prime} l_{\ell} \varepsilon$ 'Larve, Raupe'; lot : l'it-lot 'schlaff, kraftlos'; mog 'Tat, Geschäft, Sache', PO mog, wot.j. mug; moindni 'Märchen erzählen', PO mod, wotj. mad'-kịl; mores 'Brust', PO móres, wotj. mures; mort 'Mensch', PO mort, wotj. murt; moz:me m. 'nach meiner Art', wotj. muzen; moz : moz-dor 'Schoss'; no 'nun', PO no; nog: me nogen 'nach meiner Art'; noi 'Tuch, Wollstoff', PO noj; nollini 'tragen, schleppen', PO nólli-,

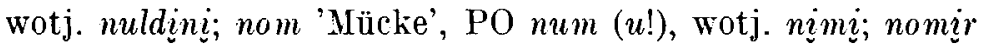
'Wurm, Made', wotj. nomîr, numir; nomsaśni 'besondere Gelüste haben (schwangere Frauen)'; PO (LrTk.) nort 'длинные охотничьи салазюи'; PO (Lүтк.) not- 'вести, тащить': PO nóốlaś- 'sich recken', wotj. naziljan?̣̂; PO ńókera 'krumm', wotj. nàkị̂res; nolzíni 'durch Schweiss oder Feuchtigkeit verderben (intr., Haut zwischen den Beinen)'; norni 'sich neigen, schief werden'; og, on, oz Verneinungsverb, $\mathrm{PO} o g$, on, oz, wotj. $u g, u d, u z$; PO ókti- 'abhauen, fällen'; olni 'leben, sein', PO ól-, wotj. ulịn $\hat{\imath}$; oltsalni 'helfen', PO ótsal-; ord : ord-li 
'Rippe', PO ord-li, wotj. urd; oš 'Bär', PO oš; polddini 'aufschwellen, anschwellen', PO pol, wotj. puli, puldịn $\hat{\imath} ;$ pol $\varepsilon$ 'Lockruf für Schweine'; PO (LYTk.) polit 'тладкий'; pon 'Ende, Anfang, Spitze', pondini 'beginnen, anfangen', PO pon, póndi-, wotj. pum, puฑ; ponel 'junge Fichte oder Kiefer'; PO pórjot- 'begrüssen, gratulieren'; porkjalni 'stolzieren'; porś 'Schwein', PO pors', wotj. pars'; pos 'Brücke', PO pus (u!); posni 'klein, fein', PO pósnii; potni 'bersten, platzen', PO pót-, wotj. put; poț̣s 'Stange', PO poč, wotj. putș̆; pòz 'Nest', PO poz, wotj. puz; poźalni 'gefühllos werden (Hand, Bein); poźni 'möglich sein', PO póź ; požęm 'Kiefer', PO póżem, wotj. puž̂ิm; požjalni 'spülen, ausspülen (Wäsche)'; rok 'Brei,

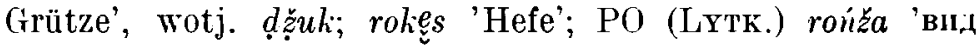
птицы'; so 'sieh da!'; PO (Lүтк.) sogra 'болотистый лес'; РО) soj 'Schwester'; sorni 'mischen, mengen', PO sóral-, wotj. surani; sors 'Kamm des Hahnes oder der Henne', PO sors; sotni 'brennen, verbrenmen (tr.)', PO sót-, wotj. sut?̣n î; sot's 'Schwester'; stola : lim-stola 'Schneeweihe'; škol 'Eingeweidewurm', wotj. kel; śor 'spät', PO sor; PO (LYTk.) sord : s. pelat' 'за;ние полати'; PO śórni 'Rede, Gespräch'; PO śortn'i 'Rübe'; šogalni 'traurig sein', PO šog, šógal-, wotj. šug; šogmini 'taugen', PO šógmi-; šonalni 'warm werden', šondi 'Sonne', šondini 'wärmen', šonid 'warm', PO šónal-, šóndi, šónot, wotj. šunani, šund?̂, šunịt; šor 'Bach', PO šor, wotj. šur; PO to 'da, sieh!'; tobon' : tser-tobon' 'Schlinge am Gürtel, die zum Tragen von Sachen dient'; tobraz: luk-tobraz 'Zwiebelstrauch $(5-6$ an demselben Stil)'; togjini 'zappeln (Fisch)', wotj. tug?̣̂? ; toman 'Schloss', wotj. tungon; tongini 'mit den Kirchenglocken lä uten'; topid 'dicht, fest', topedni 'zusammenfügen', wotj.

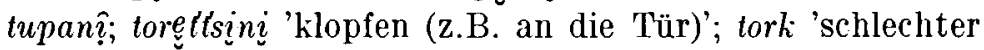
Flachs', PO tork; torkni 'stören'; PO tórket- 'klopfen'; totşskini 'einmal schlagen'; PO (Lттк.) t'solka 'вьюнок (насекомое, живущее в луже)', t'sotni 'hinken', PO cót-, wotj. tśutịn?̣; PO ćóckiom 'weiss'; tsoz 'Grossvater (mütterlicherseits)', PO(LYTK).

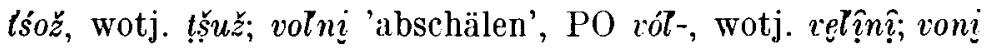
'kommen', volini 'kommen, ankommen', PO vu= (u!), wotj.

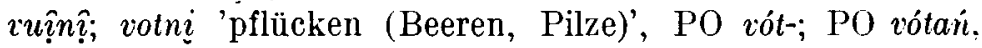
vótan : ćérañ $r$. (LYTK.) vot : t'serañ- $v$. 'Spinnengewebe', votalni 
'tropfen', PO vot' vož : kok-vož' Zwischenraum der Beine', PO vóżjolt-; zon '(unverheirateter) Bursche', $\mathrm{PO} z o n$.

Oben sind insgesamt etwa 150 syrjänische $o$-Wörter aufgezählt worden. Ungefähr 55 von ihnen haben im Wotjakischen eine regelmässige Entsprechung mit $u$. In den wotjakischen Mundarten findet sich oft eine Palatalisierung des $u$ zu $u$ (aus verschiedenen Gründen, die Wichmasx Wotj. Vok. 9-14 behandelt). An unregelmässigen Entsprechungen begegnet uns am häufigsten $a$, das in 7 wotjakischen Wörtern vorkommt: baśtịn, dzar, kam, lajị̂n parś.

Bunrich äussert in seinem Aufsatz $»$ к вопросу о пермском вокализме» (Бюллетень ЛОІКФУН 4, 1929) die Meinung, das syrj. o vertrete gegenüber dem wotjakischen $u$ eine ältere Stufe. Einige türkische Lehnwörter und solche russische Lehnwörter wie wotj. kuso 'Sense' < russ. kosa beweisen, dass sich der Lautwandel $o>u$ in der ersten Silbe im Wotjakischen verhältnismässig spät vollzogen hat. Auf denselben Standpunkt stellt sich auch Steinitz Vok. 126. Die Sache verhält sich auch ganz deutlich so. Die obenerwähnten wenigen Wörter, die im Wotjakischen eine $a$-Vertretung aufweisen, sind sicherlich so zu erklären, dass sie noch im Altwotjakischen ein o gehabt haben, das sich vor dem allgemeinen Lautwandel $o>u$ sporadisch zu $a$ geöffnet hat.

a) $o<$ vorperm. ${ }^{*} e$ : olni (304), ? korni (309), poz (327), požem (328), t'sož (335), kolni (345), ord- (348), ? mort (349). Wir haben also 6-8 Beispiele. Mit Ausnahme des Wortes loolni sind alle diese Wörter solche, bei denen im Vorpermischen in der zweiten Silbe *ä gestanden haben muss.

b) $o<$ vorperm. * $\ddot{a}$ : lokni $(252$; im syrjänischen L-Dialekt haben wir die unregelmässige Entwicklung $o>a$, im Wotjakischen den ebenfalls unregelmässigen Lautwandel ${ }^{*} u>i$ als Folge der besonders starken Expansionsneigung, die das ? im Altwotjakischen gehabt hat), pon, pom (263), oktini (281). $\mathrm{Zu}$ den ursprünglichen $* \ddot{a}$-Wörtern dürfte auch joś (288) gehören. Das Verhältnis zwischen den Schlüsseldialekten weist im Lautbestand dieses Wortes eine Inkonsequenz auf: SO joś $\sim$ PO joś. Wenn wir von *ä auszugehen haben, ist die 
Vertretung in der PO-Mundart zweifellos ursprünglich. Also beläuft sich die Zahl der Beispiele auf insgesamt 2-4.

c) Entweder auf $*_{e}$ oder auf * $\ddot{a}$ geht das $o$ in den Wörtern $l o p$ (299) und lol-pu zurück (300; die Lautgestalt der tscheremissischen Entsprechung dieses Wortes ist ganz verworren, was bei Baumnamen gar keine ungewöhnliche Erscheinung ist, aber dass es sich hier um einen ursprünglichen Vordervokal handelt, wird doch mit Hilfe des tscheremissischen Wortes ersichtlich). In diesem Zusammenhang sei auch darauf aufmerksam gemacht, dass die Formen des syrj. Verneinungsverbs, og, on, oz, hinsichtlich ihres Stammvokals kaum mit den entsprechenden mordwinischen und tscheremissischen ${ }^{*} a$-Verben (66) zusammengestellt werden können; besser passt als Vergleichsobjekt für dieses $o$ das fi. $e$ - oder das liv. $\ddot{a}$ -

Die oben aufgezählten Fälle enthalten 12 sichere Beispiele

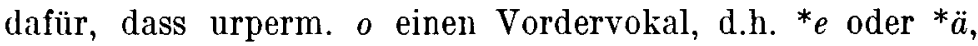
fortsetzt. Man möchte glauben, dass es sich, falls die Entwicklung $o<{ }^{*} \ddot{a}$ verlaufen ist, um einen sporadischen Überyang in die ${ }^{*} e$-Reihe handelt. Die Entwicklung ${ }^{*} e>0$ dürfte im Zeichen der Velarisierung stattgefunden haben, wobei wahrscheinlich frührperm. ${ }^{*}$ die Zwischenstufe gebildet hat; rgl. auch Fokos-Fucus, Nyr. IXXVI 45.

d) Ein Beispiel für einen gelegentlichen Übergang von $*^{*} i$ in die ${ }^{*} e$-Reihe haben wir in potș, poțšni (375). Da wir auch in tscherO $p \grave{e} \cdot t\left(\grave{s} \grave{a}, p e_{t} t \grave{s} e \cdot m\right.$ ein Ergebnis des Lautwandels $i>\boldsymbol{e}$ vor uns sehen, scheint hier ein Beweis dafür vorzuliegen, dass $t s \xi$ - wie $r$ - von Natur auf den vorhergehenden Vokal im Sinne eines Offenwerdens eingewirkt hat.

Einige wenige Beispiele gibt es auch dafür, dass urperm. o einen vorperm. Hintervokal vertritt. Wir können sie als sporadische, vereinzelte Fälle ansehen.

e) $o<$ vorperm. ${ }^{*} a$ : śorni $(38)$.

f) $o<$ vorperm. ${ }^{*} o$ : nort (141), voz (148). Wahrscheinlich gehört hierher auch sỵrj. (auch SO PO) pors' 'Schwein', wotj. parś (sporadischer Lautwandel $o>a$ ror $r$ ). Dies Wort ist eine arische Entlehnung wie auch fi. porsas und mordw. purtsos, die den gleichen Ursprung haben. Ein unsicherer Fall ist votni (114).

Es dürfte wohl offensichtlich sein, dass bei den unter e) 
und f) angeführten Fällen das $o$ sich aus einem früheren $*_{0}$ (darüber s. unten, Kapitel 3) durch sporadisches, namentlich vor $r$ und $z$ eingetretenes Offenwerden entwickelt hat.

g) Nur ein paar unsichere Beispiele gibt es für einen Lautwandel $o<^{*} u$ : jon (172), moind, mod' (190). Die Entwicklung von vorperm. ${ }^{*} u \mathrm{zu} o$ ist so ungewöhnlich, dass wir schon deshalb allen Grund haben, diese Etymologien.zu bezweifeln. Beim zweiten der soeben genannten Wörter könnte man allerdings auch an ein vorperm. ${ }^{*} o$ denken.

Über einige Wörter, bei denen sich auf Grund der verfügbaren Angaben nicht sagen lässt, ob in ihnen ein urperm. o oder $o$ gestanden hat, s. unten, SS. 284-285.

3. Syrj. (im allgemeinen) $o, \mathrm{SO} o, \operatorname{PO} u(u) \sim$ wotj.

a) $u(u)$, b) $u$ a.

Von dem geschlossenen o der Mundart der Ober-Sysola und der altsyrjänischen Sprachdenkmäler, das UotiLA durch $o$, LYTKıN dagegen im Jahr 1930 mit 0, im Jahr 1952 mit $\hat{o}$ wiedergibt, war schon oben auf S. 267 die Rede. Lytkin erwähnt (Сборник 33) die Tatsache, dass SO o allmählich aus der Rede der jüngeren Generation verschwindet. Uotila hat auch im Dialekt der Unter-Sysola (SU) neben dem $o$ ein $o$ festgestellt, aber zwischen diesen Vokalen wurde kein folgerichtiger Unterschied gemacht, sondern der betreffende Gewährsmann konnte sogar in demselben Wort bald den einen, bald den anderen von ihnen aussprechen. Das o der Mundart von Ižma hat Wichmans häufig durch $\varrho_{\imath}$ wiedergegeben, aber auch hier sind $o$ und $o$ durcheinandergemischt worden.

Lytkin veröffentlicht Соорник 32-33 und Труды I 76-78 ein Verzeichnis der $o$-Wörter im SO-Dialekt; im letztgenannten Werk sind auch die Belege des PO-Dialektes berücksichtigt worden. Uotilas Aufzeichnungen aus dem SO-Dialekt enthalten ein ebensolches Verzeichnis, das, ergänzt durch einige nur bei Lytkin vorkommende Wörter und durch die von Genetz und Lytkin erwähnten Entsprechungen im Wort- 
schatz der PO-Mundart, folgendermassen aussieht (Wörter mit einem sekundären, aus ${ }^{*} \dot{a}$ entstandenen $o$ sind hier nicht aufgezählt):

dod' 'Schlitten', PO dyd', wotj. ded

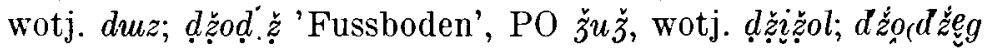

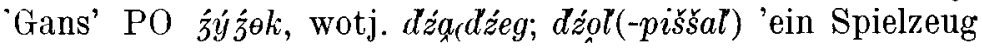
der Kinder'; dzólgini 'rieseln (Wasser)'; dzón 'unverletzt, ganz', PO źun; gob 'ein Pilz', wotj. gubi; goletettśini 'poltern'; gońjalni 'den Hals emporrecken, ausspähen'; gor (LчTk.) 'фur ypa ири пгре в лодышюи, лодышка лежит на спнне'; gors 'Höhlung im Baum'; goz 'Paar', wotj. kuz; jodmini 'alt, hart, trocken werden (z.B. Brot)'; jokišs 'Barsch'; jol 'kleiner Fluss'; jolni 'immer dasselbe sprechen'; jorni 'blenden'; jorttšini 'schwören'; kobni 'aufhören (Regen)'; kod 'betrunken', PO kud, wotj. kuddẓ̇̂n ; kodi 'wer', kor 'wenn', PO kúdik; kod' 'ähnlich', PO kud, wotj. kad; kòdș́ (3. Pers. Sg. Präs.) 'gackern'; kocdźul 'Stern', wotj. kiźili; koddźul 'Ameise', wotj. kuźiti; koini 'giessen', wotj. liujan?̧; kojok, koj (LYTK.) 'охотничья лопатка', PO (ТҮтк.) koj (o!), wotj. kuj; koitni 'balzen'; kokera 'die Wurzeln eines umgefallenen Baumes'; kol 'Zapfen', PO kuli, wotj. kulţ; kolk 'Ei, Hoden', PO kulk; kollini 'begleiten', PO kúlli-, wotj. kelni; kolni 'bleiben, zurücklassen', PO kúl-, wotj. kị̂ṭ̂n̨; kolta 'Garbe', wotj. kulto < tschuw.; komęl 'Teigklumpen'; ko-mign 'dreissig',

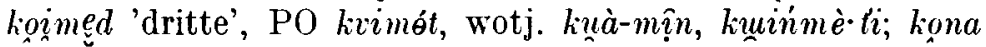
(killg) 'es erdröhnt (z.B. ein dürrer Baum)'; konjalni '(einem) ein Bein stellen'; kondini 'abgiessen, abschöpfen'; konerer 'arm', wotj. kuaner; kopirtni 'schräg stellen (z.B. einen Zuber)', wotj. kup?̂rtîn?;; kor (Lүтк.) 'шелуха', wotj. kur; kor 'Blatt (an Bäumen)', PO kur, wotj. kuar; korpa 'ein Knochen im Rücken des Vogels'; PO kưrśiž- 'gestossen oder verletzt sein'; los 'Kreuz (am menschlichen Körper)', PO kusk, wotj. kus; losni : pizzjis kosis 'sein Boot stiess auf Grund'; koś 'Schlägerei'; kośkęm-mu 'Brachacker'; kośmini 'trocken werden', wotj. kuas; kośalni 'abreissen, abziehen', PO kúsolt-, wotj. keśn̂ñ̂; kot : kod d'źul-k. 'Ameisenhaufen'; koźin 'Hochzeitsgeschenk', PO kýżin, wotj. kuẓ̇̂m; lol 'Atem, Geist', PO lul, wotj. lul; lomalni 'brennen', lontini 'heizen', PO lúnti-, ? wotj. 
lumit; mol (Lyтк.) 'лес, сплавляемый однночными орревнами по маленьким речкам'; PO múl( $(i)$ : $\operatorname{ter}(\theta)$-m. 'Moosbeere', wotj. mulị ( syrj. im allgemeinen mol' Perle', turi-m. 'Moosbeere'), dieses Wort ist vielleicht etymologisch mit dem vorstehenden zu verknüpfen; mon' 'Schwiegertochter', PO mun', wotj. men' i,tsi-m.; mortal- (LrTk.) 'messen' (= syrj. im allgemeinen murtalni), wotj. mertanî; PO nud 'Griff' (= syrjWied. nod), wotj. nid; nop 'Sack, der auf dem Rücken mittels Tragriemen aus Birkenrinde getragen wird', PO nup, wotj. nîp? ; norašnn 'sich beklagen', PO núraś-; noìdmini 'sich abnutzen (das Tuch)'; nol 'vier', PO nul, nul', wotj. riul', nill; non' 'Brustwarze'; PO ńu ̌ 'entlang; gemäss', núčtem 'unschön, hässlich' (= P ńož,

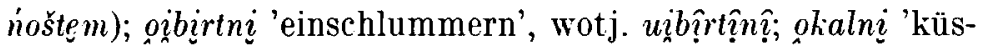
sen', PO ukal-; oktini '(eine Falle) aufstellen'; omel 'schlecht', PO i mel; omlalni 'heulen (Hund, Wolf)'; on 'Schlaf', PO un, wotj. um, un, ?̂m; onlan' 'nach unten, abwärts'; or 'Eiter', PO ur, wotj. ur; orni 'zerreissen (intr.)', PO úr-, wotj. urịn

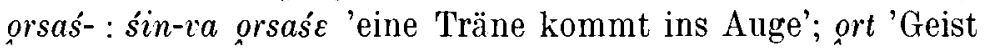
eines Verstorbenen', wotj. urt; oškini 'rühmen', PO úški-,

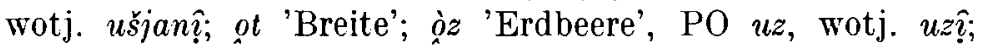
ozir 'reich', wotj. uẓ̂̂ < ar.; poden 'zu Fuss', (LxTk.) pod 'основание', РО pud, wotj. pîd, pudo; polni 'fürchten', PO púl-, wotj. puuini; bol : va-b. 'Blase', wotj. puli; pon

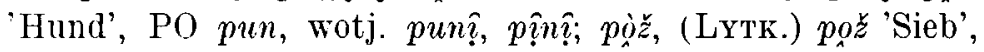
PO puž, wotj. puž; rodni (LYTk.) 'стесняться'; roi 'Baum-

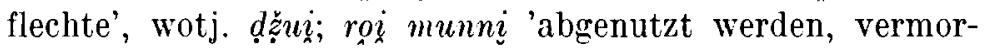
schen'; ros 'Badequast', PO rus; rots's 'Russe', PO (LYTk.) rut's', wotj. dźu t's < ostseefi.; roz 'Blüte, Traube'; ròź, (LYTk.) roź 'Loch' (dial. auch ruź); sobaln $i$ 'waten (z.B. im Morast, im Schnee)'; sod 'Leiter'; sodni 'sich vermehren', PO súd-; sod $d^{2 \prime} \dot{z}$ 'die beiden hohlen Hände zusammengehalten', PO syź, wotj. sudź; soi 'Arm', sos 'Ärmel', PO sus, wotj. sui, sajes; sol 'Salz', PO sul, wotj. sịlal; PO sür- 'stossen' (= syrj. im allgemeinen sorn $\boldsymbol{i}$ 'mischen, mengen, vermischen'); sośsinin (LYTk.) 'не прнзнаваться' (dial. auch suśkiniz), PO syśki’; śo (so) 'hundert', wotj. śu; śoi 'Lehm, Ton', PO śyj, wotj.

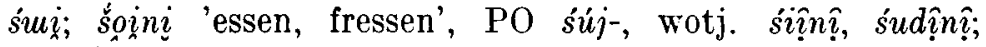
solett'š̉ini 'klappern, rasseln'; śolkedni 'verschütten (Wasser)'; 
šor 'Sparren (in der Stube)', PO śur, wotj. śurị̂; šobdi 'Weizen', PO šúgdi; šodź 'Sparren (im Speicher)'; šoi 'schlechtes Kleidungsstück oder schlechte Fussbekleidung; Leiche, Aas', wotj. šę; šottšini 'ruhen', PO šýćci-, wotj. šutätśl-; šom 'Kohle', PO šum, wotj. šum; šom : ńań-š. 'Teig', PO šym. wotj. šumes : nan'-š. 'Backtrog'; šot's 'licht, undicht', PO syć; tolun (Lyтк.) 'heute', PO ta-lun; toi 'Laus', PO tyj, wotj. tei; toini 'zerstossen, stampfen', PO tijj-, wotj. tujîn?; tom 'jung', PO tum; tomlalni 'zusammenwickeln, in Falten legen (Kleidungsstück, Tuch, Laken usw.)'; PO túņ̧̊i 'neulich'; tor 'Stück (z.B. Brot)'; toš 'Bart', PO tuš, wotj. tuš; tsoi 'Hügel'; t'śom 'Spielhäuschen der Kinder', PO ćum: t'sorid 'hart, fest', PO cúret, wotj. t'surị̂t; t'sož 'geschwind, schnell'; vo, (LYTK.) o 'Jahr', PO $u$, wotj. una(-pum); vòd $d n$ i 'sich legen', PO vúd-, wotj. vị̂?̣̣̂̂; vòddz, (LYTK.) odź 'früh'. PO uj, wotj. uaź; vọd az; oi 'Nacht', PO yj, wotj. ui; voidar-lun, (LYTk.) oidorlun 'vorgestern', PO odér-lun; oitir 'Leute, Volk', PO ýter, wotj. otyr; vol' 'Bruder'; ol-(paś) 'Bett', (Lүтк.) oles 'подстилка', P() ul-(pas), wotj. ual?̣̂n̂, uales; vollalni 'glänzen'; voltid 'glatt, schlüpfrig', PO vólkot (o!); omenn 'quer', wotj. uamen; vomdźalni 'durch den bösen Blick bezaubern', PO vómiź; PO vun 'Bruder' (= syrjP von), wotj. vịn; orga: šor-o. 'Bachbett': orop 'Stiel, Griff', $\mathrm{PO}$ urp, wotj. varyp; orsni 'spielen', $\mathrm{P(}$ úrs-; PO uśskol 'Schritt' (= syrj. im allgemeinen vośkol), wotj. utśkyl; ośtini 'öffnen', PO uśt-, wotj. uśtîn (= syrj. im allgemeinen vot), wotj. vit; $\mathrm{PO}$ vuc- 'ausbessern. flicken' (syrj. im allgemeinen votśni, reftśni); oftśa 'gegenüberliegend', PO úććan, wotj. ua t'śc; òž 'Deichsel', wotj. uaj ţz; zòr 'Stange', wotj. zị̂r; zolett'śini 'klingen, klingeln (z.B. Münzen'); žodźmini 'einen üblen Geschmack bekommen (Milch)'; zol'kmunema (sur) (Lүтк.) 'прокисло (пиво)'; РО žn' 'Finkbeisser, Dompfaff' (= syrj. im allgemeinen žon').

Die Zahl der $o$-Fälle ist im Syrjänischen ziemlich genau dieselbe wie die der $o$-Wörter, nämlich etwa 150 . Ungefähr 70 davon haben im Ostpermjakischen Entsprechungen, in denen dem $o$-Vokal $u$ bzw. $y$ entspricht. Das $y$ bedeutet den Mittelvokal $u$, vgl. S. 293. Die Entwicklung ist im Ost- 
permjakischen $o .>u$ und unter bestimmten Bedingungen weiter $>u$ verlaufen. Die Palatalisierung des $u \mathrm{zu} u$ ist offenbar lautgesetzlich in solchen Fällen eingetreten, wo auf den Vokal $j(i)$ folgt oder gefolgt ist; dafür haben wir 7 Beispiele. Ausserdem gibt es 4 Beispiele für eine Palatalisierung des $u$ vor einem mouillierten Konsonanten, was sporadisch ist, denn gewöhnlich hat sich $u$ vor solchen Konsonanten erhalten (in über 15 Fällen). Etwa 50 syrjänische $o$-Wörter haben im Wotjakischen Entsprechungen mit $u$ bzw. $u$. Ein Sonderfall, der aber an sich als lautgesetzlich anzusehen ist, ist die Vertretung $u a$, der wir in 10 wotjakischen Wörtern begegnen. Ausserdem treten im Wotjakischen vereinzelte unregelmässige Fälle auf. Diese sporadischen Entsprechungen des syrj. o haben im allgemeinen eine recht geringe Frequenz. Die gewöhnlichste von ihnen ist $\underset{i}{i}$ für das es (zum Teil neben normalem $u$ ) 15 Beispiele gibt. Es ist offensichtlich, dass das $i$ auf ein $u$ zurückgeht, das im Frühurwotjakischen noch vorkam. Vgl. SS. $318-319$.

Wie Verf. im Virittäjä 1951 S. 446 und Lrtкıs in Tруды I S. 76 gezeigt haben, hat es schon im Urpermischen neben $o$ ein $\varrho$ gegeben, das also in den heutigen syrjänischen Mundarten im allgemeinen mit dem urperm. $o$ zusammengefallen ist; am besten hat es sich im Dialekt der Ober-Sysola von diesem getrennt erhalten. Im Ostpermjakischen und im Wotjakischen ist das $a \mathrm{zu}$ $u$ geworden, aber das aus ostpermjak. $o$ entstandene $u$ der ersten Silbe unterscheidet sich rom ursprünglichen $u$ dadurch, dass es in zwei- und mehrsilbigen Wörtern betont ist, rgl. unten S. 293. In den Wörtern, die im Wotjakischen ein $u a$ aufweisen, können wir ein urperm. * ${ }_{n}^{*} o$ annehmen. Im Folgenden behandeln wir die Wörter mit $o$ und die mit *uo als getrennte Gruppen.

\section{A. Urperm. $o$.}

a) Auf vorperm. ${ }^{*} e$ geht das $o$ in folgenden Wörtern zurück: loor (308), ? kos (311), lol (315), moš, PO -muš (im syrjänischen V-Dialekt wider Erwarten $u$ : -muš) (317), nol (322), polni (324), pon (325), śsoini (336; im Wotjakischen sporadisch 
$u>i$ : siộn $n \hat{q}$ aber die lautgesetzliche Vertretung hat sich in der Ableitung sudîn $\hat{\imath}$ erhalten). Beispiele haben wir insgesamt 7-8. In 5-6 Wörtern hat nach dem Zeugnis der verwandten Sprachen in der zweiten Silbe ${ }^{*} e$ gestanden, in zwei Wörtern (nol', pon) * $\ddot{a}$. Es ist jedoch offensichtlich, dass das $o$ in nol nach dem Vokal des Zahlworts für 'drei' (im Frühurpermischen vermutlich ${ }^{*} k_{0} l m$ oder ${ }^{*} k_{n o l} l m<<{ }^{*}$ kolm) analogisch eingesetzt worden ist; die primäre Lautvertretung hat sich in der Ableitung nellà-min ' 40 ' erhalten. Somit bleibt als Beispiel für $o$-Vertretung in einem ursprünglichen * $\ddot{a}$-Stamm nur pon. Berücksichtigt man, was oben in Punkt a) des 2 . Kapitels (S. 271) gesagt wurde, so kann man als sicher ansehen, dass vorperm. ${ }^{*} e$ im Urpermischen vor in der zweiten Silbe stehendem $* \ddot{a} \mathrm{zu} o$, aber vor $* e$ weiter zu $o$ geworden ist. Kann man daraus schliessen, dass noch im Frühurpermischen ursprüngliches $* \ddot{a}$ in der zweiten Silbe deutlich von ursprünglichem ${ }^{*} e$ unterschieden wurde? Möglicherweise hat es sich so verhalten; das Frühurpermische kann hinsichtlich der Vokale in der zweiten Silbe eine sehr viel konservativere Sprachform gewesen sein als das Späturpermische, in dem die Vokalunterschiede, die die Zweiteilung des ursprünglichen ${ }^{*} e$ verursacht hatten, zweifellos schon völlig ausgeglichen waren. Es muss aber auch die Möglichkeit zugegeben werden, dass der Unterschied zwischen $o$ und $o$ in irgendeiner Form bereits in die vorperm. Zeit zurückreicht. Im Lappischen hat sich nämlich das ursprüngliche ${ }^{*} e$ ganz parallel zu den Lautentwicklungen im Permischen vor in der zweiten Silbe stehendem * $\ddot{a}$ zu einem offeneren Vokal entwickelt (im Frühurlappischen ${ }^{*} \varepsilon$ ) als vor ${ }^{*} e$ (in letzterem Fall dürfte im Frühurlappischen etwa ${ }^{*}$ eingetreten sein), rgl. z.B. urperm. ${ }^{*}$ ol-, lp. allet 'leben' $<{ }^{*}$ elä- $\sim$ urperm. ${ }^{*}$ pol-, lp. bâllât 'fürchten' $<^{*}$ pele-. Vielleicht deutet dieser Parallelismus darauf hin, dass das ${ }^{*} e$ wenigstens in einem Teil des fi.-ugr. Sprachgebiets schon sehr früh seinem "absoluten" Lautwert nach (d.h. in einsilbigen Wörtern und mit einem zweiten $* e$ kombiniert in zweisilbigen Stämmen) um einiges fester artikuliert wurde als z.B. das fi. $e$. Falls es sich so verhalten hat, würden sich nicht bloss das urperm. $o$ und $o$ in ihren verschie- 
denen Entwicklungsstufen als Laute erweisen, die den alten kombinatorischen Gradunterschied treu bewahrt haben, sondern auch die im Lappischen teilweise vor sich gegangene Entwicklung des $e$ zu einem sog. dunklen $a$ zusammen mit den geschlossenen Vokalen $i$ und $\ddot{u}$ würde eine natürliche Erklärung erhalten. Fokos-Fuchs hat seinerseits angenommen, dass fi.-ugr. ${ }^{*} e>$ syrj. $o$, fi.-ugr. "offenes $e »\left(\right.$ dh. ${ }^{*} \ddot{a}$, bei Fuchs $\left.{ }^{*} \varepsilon\right)>$ syrj. o, s. Nyr. LXVI 42-46.

b) $o<$ vorperm. *a: olktini (1), vośkol, PO úśkol (7), koln (14), ? koini (16; lp. goailiot deutet nicht auf *a, sondern auf $*_{o}$ hin), goz (20), śor (42), ozir (68; in L unregelmässiges $u$ ), koziin (71), roź (84; VL U I unregelmässiges $u$ : ruź). Insgesamt haben wir $8-9$ Beispiele.

c) $o<$ vorperm. *o: vòd ${ }^{o}$ (113), pò (137); insgesamt sind es 4 Fälle.

Die eigentliche Fortsetzung von vorperm. ${ }^{*} a$ und ${ }^{*} o$ in den permischen Sprachen ist das urperm. $u$, s. SS. 286-288; vgl. auch Gouвocz NyK XXXIX 250, 264, Lyтkix Tруды I 62. Die oben unter b) und c) aufgezählten Wörter widerspiegeln sporadische Übergänge des urperm. ${ }^{*} u$ oder der Vorstufe desselben zum qualitativ benachbarten $o$-Vokal, der nur als Fortsetzung von vorperm. ${ }^{*} e$ als lautgesetzlich anzusehen ist. Wie oben bei den Punkten 2 e-f) (SS. 272-273) erwähnt wurde, hat sich das auf einen ursprünglichen Hintervokal zurückgehende $*_{0}$ selten, namentlich vor $r$ und $z$, zu $o$ geöffnet.

Gelegentliche vereinzelte Fälle:

d) $o<$ ? vorperm. *u: kot (175). Leichter als eine Entwicklung $*_{u}>*_{0}$ könnte man annehmen, dass bei diesem Wort die Form mit $u\left({ }^{*} l i u t k e\right)$ schon früh eine Nebenform mit $o$ gehabt hat.

e) $o<$ ? vorperm. ${ }^{*} \ddot{u}$. Auf diese Weise sind möglicherweise sodni (424) und oi (436) entstanden. Bei oi lässt sich jedoch eine auf $* \ddot{u}$ zurückgehende Lautvertretung erst in der ostseeund wolgafinnischen Gruppe feststellen, während die permischen und ausser ihnen noch die ugrischen Sprachen auf einen illabialen Vokal hindeuten. Es ist auch nicht sicher, ob das ï der fi. Entsprechung von sodni, nämlich des Wortes syn- 
tyä, ursprünglich ist. Eine im Frühurfinnischen vor sich gegangene sporadische Labialisierung *sentü- $>$ süntü- könnte die eigenartigen Lautverhältnisse erklären.

f) $0<{ }^{*} \bar{o}$. Ein Beispiel für eine derartige Lautentwicklung ist wahrscheinlich rot's (159). Hier handelt es sich jedoch nicht um eine Fortsetzung des vorperm. ${ }^{*} \bar{o}$, sondern des ostseefi. $*_{\bar{o}}\left(\right.$ ? $\left.*_{u o}\right)$. Die ostseefi. Entsprechung von syrj. sol (161) hat $\bar{o}$, aber die Lautverhältnisse dieser Wortfamilie sind bekanntlich verworren, so dass man nicht ohne Grund gefragt hat, ob die in den verschiedenen Sprachen gebräuchlichen Wörter direkt aus derselben Grundform stammen können. Im wotj. silal liegt offenbar ein sporadischer Lautwandel $u>i$ vor.

g) $o<$ ? vorperm. *ä: syrjP von, PO vun (278). Die Zusammengehörigkeit dieses Wortes mit fi. vävy ist unsicher, aber nicht unmöglich. Es sei darauf hingewiesen, was bereits oben (S. 221) über die lautliche Buntheit in dieser Wortfamilie gesagt wurde. Die wotj. Formen vin, vîn zeigen wieder einen sporadischen Lautwandel $u>i$.

\section{B. Urperm. *uo.}

Das obige Verzeichnis der $o$-Wörter enthält eine ganze Menge solcher Wörter, in deren wotjakischen Entsprechungen statt des normalen $u$ die Lautverbindung $u a$ vorkommt. Sie ist entweder im Anlaut oder nach $k$ anzutreffen. Bei den syrjänischen Entsprechungen der mit $u a$ anlautenden wotjakischen Wörter sehen wir in den meisten Mundarten ein sekundäres $v$ im Anlaut; z.B. syrj. (im allgemeinen) vol' 'Renntierhaut, Bett, usw.', SO ol', P ol', PO $u l^{\prime}$, wotj. ual'-; syrj. (im allgemeinen) kor 'Blatt' SO kor, PO kur, wotj. kuar. Diese Fälle hat UotruA eingehend und sehr verdienstvoll in seinem Werk »Zur Geschichte des Konsonantismus in den permischen Sprachen", SS. 14-16, 63-70, behandelt. Man kann hier die urperm. Lautentwicklung $*_{0-}>u_{0-}, *_{k_{0}-}>*_{k} u_{0}-$ annehmen. Der Halbvokal $u$ hat sich im Wotjakischen erhalten, während der darauffolgende Vokal sich zu a geöffnet hat; 
im Syrjänischen hat sich das anlautende $\underset{\sim}{u} \mathrm{zu} v$ weiterentwickelt, das im grössten Teil des syrjänischen Sprachgebiets erhalten geblieben ist, während im Inlaut und mundartlich auch im Anlaut das $u$ oder der aus diesem entstandene Laut geschwunden ist. Die Vertretung des auf das $u$ folgenden $o$ ist im Syrjänischen überall ebenso wie in den Fällen, wo sich kein $u$-Vorschlag herausgebildet hat. Dies $u$ erscheint niemals vor einem urperm. $o$.

Über die Laute, die im Vorperm. die Vorgänger des urperm. * uo gewesen sind, gibt das spärliche Material nur wenige Hinweise:

a) ${ }^{*} u_{0}<$ vorperm. ${ }^{*} 0$ : $k o-m i n$, wotj. kuamin (95), kor, wotj. kuar (97), *ko, wotj. kua (99), ? koś-, wotj. kuaś (133; ausser den perm. Sprachen deutet noch das Tscheremissische auf *o hin, dagegen das Lappische und vielleicht auch das Mordwinische auf $* u$ ).

b) $*^{*} u_{\alpha}<$ vorperm. ${ }^{*} a$ : ? wotj. kuatṣ̆kàni (22); òz , wotj. naj? ?̧z $(69)$.

c) ${ }^{*} u_{n} 0<$ vorperm. ${ }^{*} \bar{o}:$ vo- $\sim o^{-}$, wotj. una- (167).

Obwohl es nur 5-7 Beispiele gibt, dürfte es kein blosser Zufall sein, dass in ihnen allen das *uo auf einen ursprünglichen Hintervokal zurückgeht. Wenn sich nun bei dem auf einen ursprünglichen Vordervokal zurückgehenden $o$ ein u-Vorschlag gar nicht herausgebildet hat, so verstehen wir, warum z.B. die Fortsetzung des ursprünglichen Stammes *kere, kor 'Rinde' (308) im Urpermischen nicht lautlich identisch mit kor 'Blatt' ( $<$ urperm. *kuor $<<{ }^{*}$ korva) gewesen sein kann. Oben aber haben wir gesehen, dass auch vor einem auf einen Hintervokal zurückgehenden anlautenden oder auf $k$ folgenden $o$ meistens das $u$ fehlt. Es scheint unmöglich, in der heutigen Sprache irgendwelche lautlichen Grundlagen dafür zu finden, wann sich vor einem $\underset{\alpha}{o}$, das auf einen Hintervokal zurückgeht, ein $u$ entwickelt hat und wann nicht. Man kann also lediglich Vermutungen anstellen.

Die Teilung des $o$ in $u$ und $o$ (zusammen $=u o$ ) dürfte als Diphthongierung aufzufassen sein. Darauf weist die Tatsache hin, dass sich das $u$ auch im Anlaut und nicht nur nach $k$ hat herausbilden können. Käme es lediglich nach $k$ vor, so 
liesse sich die Kombination $k u$ als Labialisierung des $k$ erklären, eine Erscheinung, die beispielsweise in den ob-ugrischen Sprachen gewöhnlich ist. (Uотіц AK 16 vergleicht auch wotj. $k u$ - und wog. $k \beta$ - miteinander.) Handelt es sich hingegen um Diphthongierung, so bedarf die Herausbildung des $u$ im Anlaut keiner Erklärung und auch nach hinterem $k$ ist die Diphthongierung des o phonetisch natürlich. Als Vergleichspunkt sei auf die diphthongische Aussprache des o nach hinteren Palatalkonsonanten im Russischen und (unter dem Einfluss der russ. Phonetik?) im Kildinlappischen hingewiesen.

Es erscheint am natürlichsten, dass es für das Auftreten oder Fehlen von $u$ vor einem auf einen Hintervokal zurückgehenden $o$ zwei alternative Ursachen gegeben hat. Erstens lässt sich denken, dass es in einer bestimmten Periode des Frühurpermischen neben dem kurzen $o$ auch ein langes $\bar{o}$ gegeben hat und dass gerade dieser Laut im Anlaut und nach $\hat{k}$. diphthongiert wurde. Dieses $\bar{o}$ dürfte nicht die Fortsetzung des vorperm. ${ }^{*} \bar{o}$ gewesen sein, denn dies ist im allgemeinen durch einen anderen Laut vertreten (s. S. 324). Die Vorstufe des $u_{n}$ hat sich wohl durch eine sekundäre Dehnung. deren Voraussetzungen uns unbekannt sind, aus vorpern. kurzem ${ }^{*} o$ und ${ }^{*} a$ entwickelt, aber nur selten aus ${ }^{*} \bar{o}$, wie wir oben gesehen haben. Die zweite alternative Erklärung wäre die, dass in der ersten Silbe nur ein kurzes $o$ gestanden hätte. dessen Erhaltung oder Diphthongierung irgendwie rom Vokalismus der zweiten Silbe abhängig gewesen wäre. Da heute die Geschichte des Vokalismus der nichtersten Silben der permischen Sprachen noch völlig ungeklärt ist, vermögen wir die Tauglichkeit dieser zweiten Erklärung in keiner Weise zu beurteilen. Sehr unwahrscheinlich ist jedenfalls, dass die Erhaltung oder Diphthongierung des $o$ durch Betonungswechsel verursacht gewesen wäre. In einigen Fällen, in denen man im Syrjänischen ein vor $o$ sekundär entstandenes $r$ antrifft, nimmt Lytkis an, dass dies $v$ sich aus der Anfangskomponente eines früheren uo-Diphthonges entwickelt hat. und hält es für möglich, dass das diphthongierte $o$ betont. das monophthongisch gebliebene dagegen unbetont gewesen ist (Древнепермскпй язык 99). Fast alle von Lytkin ange- 
führten Fälle gehören jedoch zu denen, wo ein auf den Vokal folgendes $i$ oder ein palatalisierter Konsonant eine Palatalisierung des $o$ hervorgerufen hat, womit noch die Herausbildung eines $v$ vor dem $o$ verbunden gewesen ist. Vgl. Uorila PK 91-92. Die Betonungsverhältnisse haben also mit dem von Lytkin betrachteten Wandel von $a$ zu vo- nichts zu tun. der als eine auf das Syrjänische beschränkte Erscheinung von der älteren, urpermischen $u o$-Bildung ganz getrennt werden muss. Lytkins Theorie wurde in diesem Zusammenhang nur erwähnt, um festzustellen, dass keine Veranlassung besteht. derartige Betonungswechsel anzunehmen. Im urpermischen Akzentuationssystem ist das $o$ der ersten Silbe i m m e r betont gewesen, s. näher S. 293.

Wahrscheinlich ist die Lautentwicklung vorperm. ${ }^{*} o,{ }^{*} a>$ frühurperm. $*_{o}\left(?{ }^{*} \overline{0}\right)>{ }^{*} u o$ älter als der Lautwandel vorperm. ${ }^{*} e>>$ urperm. $o$, der erst vor sich gegangen ist. nachdem das anzunehmende, auf vorperm. ${ }^{*}$ und *a zurückgehende frühurperm. ${ }^{*} o$ gewöhnlich zu $u$ geworden war, oder höchstens parallel mit dieser Lautentwicklung. In den Fällen aber, in denen sich vor frühurperm. ${ }^{*} o$, das auf einen Hintervokal zurückging, ein $u$ herausgebildet hatte, erhielt sich das $o$ und wurde nicht $\mathrm{zu} u$. Wenn sonst späturperm. o vorperm. ${ }^{*} a$ und $*_{o}$ vertritt, muss es sich um einen Reihenübergang handeln, wie auf S. 279 dargelegt worden ist.

Wir haben Veranlassung, noch gesondert ein paar $u$-Fälle zu betrachten. Das Zahlwort kujim 'drei', wotj. kuin'(m-). dessen Lautvertretung heute von der bei SO ko-min 'dreissig', wotj. kù̀-min abweicht, scheint auf die frühurperm. Form ${ }^{*} l_{n} l$ l'm oder *kuol'm zurückzugehen. Man beachte besonders koimed 'dritte' <*kol'med oder *kuol'med. Nach Uotila muss man bei kujim, wotj. kuin (m-) von einer urperm. Form mit $u$ ausgehen (im Wotjakischen früher *kuńm-, s. PK 16), mit anderen Worten, das anzunehmende frühurperm. ${ }_{0}^{*}(<$ vorperm. ${ }^{*} o$ ) hätte sich lautgesetzlich zu $u$ geschlossen. Aber das wotj. ku- im Anlaut, das leicht eine palatalisierte Variante des obenerwähnten $k \mathfrak{r}$ - sein könnte, zwingt uns doch, zu fragen, ob nicht auch in kuin'(m-) und kuinmè ti eine mit kuoanlautende Form den Ausgangspunkt gebildet hat, wie es 
ganz sicher in kuàmin der Fall gewesen ist. Somit müssen wir neben der Erklärung Uotilas als Alternative die Entwicklung ${ }^{*} k u o l m>{ }^{*} k u i l m$ (sporadischer Lautwandel $a>i$ unter der Einwirkung des darauffolgenden $l$ ) $>$ syrj. kujim, wotj. kuiin' $(m-)$ für möglich halten. - Recht merkwürdig ist der Lautbestand eines anderen Zahlwortes, nämlich syrj. kvaint, kvat' 'sechs', wotj. kuat'. Wenn wir hier urperm. *kuot' anzunehmen hätten, worauf wotj. kuat' natürlich hinzuweisen scheint, wäre syrj. kvaît, kvat' das einzige Beispiel dafür, dass die Entwicklung auch im Syrjänischen ganz die gleichen Wege hätte einschlagen können wie im Wotjakischen, in dem sich $k u$ - erhalten hätte - in der Form $k v$ - - und danach $o>a$. Es lässt sich schwer sagen, auf welchen vorperm. Laut der Vokal der ersten Silbe im Urpermischen zurückgeht, denn die Vokalverhältnisse dieses Wortes in den verschiedenen fi.-ugr. Sprachen sind bunt (vgl. STEinitz Vok. 44, 55), was übrigens bei Zahlwörtern eine gewöhnliche Erscheinung ist.

Anhang zu den Kapiteln über urperm. $o$ und $o$.

Es gibt eine ganze Menge 0 -Wörter, von denen wir nicht wissen können, ob sie im Urpermischen ein o oder $o$ gehabt haben, weil sie weder im SO- noch im PO-Dialekt bekannt sind.

Als erstes sei erwähnt sozor, wotj. suzer (389). Die Verwandtschafts- und Lautverhältnisse zwischen den Mitgliedern dieser Wortfamilie sind unklar, s. oben S. 203. Wenn das permische Wort mit mordw. sazor zusammengehört, so dass wir von vorperm. * $a$ auszugehen hätten, würde es sich mit Sicherheit um ein urperm. o handeln. Falls dagegen sozor unmittelbar mit est. sõsar $\left(<{ }^{*}\right.$ sesar) zusammengestellt werden kann, müsste man vielleicht eher die Entwicklung ${ }^{*} e>>$ urperm. $o$ vor offenem Vokal der zweiten Silbe voraussetzen.

In syrjI $l \bar{o}(416)$ fällt es schwer, von vorperm. * $\ddot{u}$ auszugehen, obwohl in der fi. Entsprechung dieser Vokal steht. Berücksichtigt man noch die ostj. Entsprechung (V. Vj. l, l, usw.), die den urostj. Illabialvokal * aufweist, so kann man sagen, 
dass sich sowohl die syrj. als auch die ostj. Lautvertretung einwandfrei aus fi.-ugr. ${ }^{*} e$ erklären. Vielleicht ist das $\ddot{u}$ in fi. lyly im Frühurfinnischen durch sporadische regressive Assimilation entstanden: ? *lelü > lülü (vgl. ? *sentü- > süntü-). Über die ursprüngliche Qualität des Vokals der zweiten Silbe lässt sich heute nichts mehr sagen.

Wotj. vur (341) hat keine Entsprechung im Syrjänischen, aber um ein urperm. $u$ kann es sich hier nicht handeln. Da das Wort offenbar auf vorperm. *verä zurückgeht, können wir eigentlich keine andere urperm. Form annehmen als *vor.

Alle folgenden Wörter sind solche, in denen wahrscheinlich urperm. o gestanden hat:

a) < vorperm. ${ }^{*} a$ : vojny (2), moi $(26$; im Wotjakischen sporadisch $* u>\hat{\imath}$ ), ? nokt'sim (63; in der S-Mundart unregelmässiges $e$ ).

b) < vorperm. ${ }^{*}$ : $k o s(k)(98)$, tokti (126; dieses Wort, das zweifellos onomatopoietischen Ursprungs ist, kann natürlich getrennt von fi. tohtaja entstanden sein).

c) < ? ? vorperm. ${ }^{*} u$ : ob (213). Die Zusammengehörigkeit dieses Wortes mit dem fi.-lp. Wort, bei dem ein ${ }^{*} u$ vorliegt, ist unwahrscheinlich. Wenn die Zusammenstellung richtig wäre, müsste es sich hier wohl um einen ebensolchen dunklen Fall eines Lautwandels $* u>0$ handeln wie vielleicht in $k_{\Lambda} t$ (175), s. S. 279.

4. Syrj. $u$, PO $u(u) \sim$ wotj. $u(u)$.

Wichmann hat Wotj. Vok. 82 die Zahl der Wörter, die dieses Lautverhältnis widerspiegeln, auf etwa 140 berechnet.

Das $u$ ist gemeinpermisch und reicht somit unstreitig in die urperm. Zeit zurück. Im Ostpermjakischen ist regelmässig vor $j$ oder mouilliertem Konsonanten $u>u$ (= Genetz $y$ ) (wogegen PO $u<$ urperm. o gewöhnlich nur vor $j$ zu $u$ geworden ist, nicht aber vor mouilliertem Konsonanten, s. S. 277). Im Wotjakischen sehen wir oft den Lautwandel $u>u$, ganz ebenso wie bei demjenigen $u$, das auf urperm. $o$, o zurückgeht. 
a) $u<$ vorperm. a: ul (3), udni (5), jukni (9), kulem (19). lud (24), mus (27), bur (34), suni (37), ? suk (39), turin (47). dugdini (48), vug (54), vuďni (64; im Wotjakischen sporadisch $u>i)$, ? mudźni (72; syrjI P sporadisch $u>i$ ), sudźni (75), tšužni, t'śzzn $(77$; im Wotjakischen sporadisch $u>i)$ ), $v u \underline{z}$ (80; im Wotjakischen sporadisch $u>i$ ), juvalni (81), sulaln $i$, sultni (85; im Wotjakischen in sultini lautgesetzliches $u$ aber in $\operatorname{sil}(i) n i$ sporadisch $u>i$ ). Insgesamt sind es $17-19$ Beispiele.

b) $u<$ vorperm. ${ }^{*} 0$ : ? $j u$ (88; die lautliche Entwicklung dieses Wortes, das zu einem einsilbigen Vokalstamm abgeschliffen ist, lässt sich schwer beurteilen, und deshalb muss auch die Möglichkeit offen gelassen werden, dass das $u$ hier auf dieselbe Variante mit ${ }^{*} u$ zurückgehen könnte wie lp. jokliâ), juk (89), juś (91; dieses Wort geht deutlich auf eine Urform mit *o zurück, lp. njuklčâ dagegen auf eine solche mit *ū), kud (92), kuțş (100), lun (102), munda (103; syrj. -mịn, -minda und wotj. -minn, minda weisen einen sporadischen Lautwandel $u>i$ auf), ? $n u(l) n i$ (105), ur (111), vuz (112), sur (119), ? (dod'-)śul 'Schlittenkufe' (120), sul(-t'se éz) 'Ente' (124), śul 'Darm' (125), nur (140) țşuž, t'śž (145), tul (146). luź (150), puly(-pom) (154). (Über das $u$ von kujim 'drei' s. SS. 283-284.) Insgesamt haben wir 16-19 Beispiele.

In den folgenden Wörtern geht das urperm. $u$ entweder auf vorperm. ${ }^{*} a$ oder ${ }^{*} o$ zurück: juskini (10; es lässt sich nicht entscheiden, ob dies Wort lautlich eher dem damit zusammengehörigen fi. Wort entspricht oder dem mordwinischen), pur (35), $u(v) t n i$ (152), u兰 (153). Bei den letzten zwei Wörtern lässt sich auf Grund des Lautbestandes ihrer tscher. Entsprechungen am ehesten vorperm. ${ }^{*}$ annehmen.

Im Vorstehenden sind etwa 40 Wörter aufgeführt, in denen urperm. $u$ früheres ${ }^{*} a$ oder ${ }^{*} o$ vertritt. Diese beiden Vokale sind im Urpermischen deutlich zusammengefallen. Vermutlich wurde zuerst das *a geschlossener und verschmolz mit dem *o und danach entwickelte der so entstandene einheitliche Vokal sich weiter zu $u$, wahrscheinlich über eine frühurperm. Zwischenstufe $o$. Die Auffassung von Stescitz, dass fi.-ugr. ${ }^{*} a$ und ${ }^{*} o$ im Syrjänischen auf verschiedene Weise vertreten sind. 
nämlich $*_{a}>0$ und $*_{0}>u$ (Vok. 32, 46), hält nicht Stich. (Schon oben war die Rede von den Fällen, in denen der Laut. der vorperm. ${ }^{*} a$ bzw. ${ }^{*} o$ vertrat, offensichtlich von der $u$-Stufe zur $o$-Stufe zurückgekehrt ist und sich mit dem $\mathrm{n}$ e u e $\mathrm{n}$ $o$-Vokal vermischt hat, dessen Ausgangspunkt vorperm. ${ }_{e}$ gewesen war.)

c) $u<$ vorperm. ${ }^{*} \bar{o}$. Es gibt eigentlich nur zwei Beispiele: kulni (155), pul (158). Möglicherweise ein drittes Beispiel haben wir in rulni (193), dem aber ebensogut wie das fi. nuolla auch $1 \mathrm{p}$. njoallot $\left(o a<{ }^{*}\right.$ ) am nächsten entsprechen kann. - Der Laut, der das vorperm. ${ }^{*} \bar{o}$ eigentlich im Urpermischen vertreten hat, ist ein anderer gewesen $\left({ }^{*} \dot{a}\right.$, s. S. 298) und es bleibt dunkel, wodurch diese vereinzelten Fälle verursacht worden sind, die die Lautentwicklung $*_{\bar{o}}>u$ aufweisen.

d) $u<$ vorperm. ${ }^{*} \bar{u}$ : juni (231), pu (235). Ein unsicherer Fall ist wotj. śu (204), dessen Vokal natürlich ebenso gut auf urperm. o oder $o$ wie auf $u$ zurückgehen kann. Im Inlaut ist vorperm. ${ }^{*} \bar{u}$ gewöhnlich zu $i$ geworden (vgl. S. 302). aber es kann sein, dass in einsilbigen Vokalstämmen, wie es die soeben in Rede stehenden Fälle sind, irgendein Auslautgesetz die Entwicklung $* \bar{u}>u$ veranlasst hat. Über syrj. jur (232) und kun (239) s. S. 302. - Sowohl hinsichtlich der Geschichte des vorperm. ${ }^{*} \bar{o}$ als auch hinsichtlich der des vorperm. ${ }^{*} \bar{u}$ kann man schliesslich auf den deutlich feststellbaren Umstand hinweisen, dass ein Vokal, je kleiner seine Frequenz gewesen ist, um so geringere Möglichkeiten zu einheitlicher Entwicklung in späteren Stadien gehabt hat.

e) $u<$ vorperm. $* u$. Für eine solche ganz unregelmässige Entwicklung (vorperm. $*_{u}>$ lautgesetzlich urperm. $i$, s. S. 300) gibt es ein oder zwei Beispiele. In purni $(199 ;<$ *pure-) dürfte man zu dem $u$ gekommen sein, um Verwechslungen mit dem Wort pirni $\left(228\right.$; $<{ }^{*}$ pura-) zu vermeiden. Die Zusammengehörigkeit von syrj. vunni (214) mit fi. unohtaa ist nicht sicher. In syrj. uini (211) kann das $u$ auf ein $*_{o}$ zurückgehen, vgl. est., lp. - Im Syrjänischen scheint in einigen Wörtern urperm. ${ }^{*} k i-\operatorname{sich} \mathrm{zu} k u$ - entwickelt zu haben, vgl. S. 317. 
Als Nachbemerkung zu den Punkten d) und e) sei hinzugefügt, dass das knapp 10 Wörter umfassende Material, auf Grund dessen Stersitz (Vok. 54-56) schliesst, der von ihm angenommene fi.-ugr. "Vollvokal $u$ " werde in den perm. Sprachen normalerweise durch $u$ vertreten, teils vom etymologischen Standpunkt gesehen sehr schwach und teils mehrdeutig, ja sogar falsch gedeutet ist.

Wir sehen also, dass es sich, wenn urperm. $u$ einen anderen Vokal vertritt als vorperm. ${ }^{*} a$ oder ${ }^{*} o$, um einen vereinzelten Fall handelt.

Anhang zu den Kapiteln über urperm. o und $u$.

Es gibt einige Wörter, die nur im Wotjakischen bekannt sind und in der ersten Silbe ein auf einen vorperm. Hintervokal zurückgehendes $u$ haben. In derartigen Fällen können wir nicht wissen, ob man von urperm. $o$ oder $u$ ausgehen muss.

a) Um ein vorperm. ${ }^{*} a$ handelt es sich offenbar in $p u d$ (82). - Es sei auch auf suter, syrj. seter (86) aufmerksam gemacht. Verglichen mit der tscher. Entsprechung (urtscher. ${ }^{*} a<$ wahrscheinlich ${ }^{*} a$ ) entspricht das wotj. Wort hinsichtlich seiner Lautgestalt den Erwartungen, während man das $e$ der ersten Silbe im Syrjänischen als eine ganz unregelmässige Abweichung ansehen muss.

b) Vorperm. *o scheint der Ausgangspunkt in folgenden Wörtern zu sein: ? usi (108), pužei (130) und ńulo (151; bei diesem Wort ist allerdings auch ${ }^{*} a$ möglich).

5. Syrj. $e, \mathrm{PO} \dot{o} \sim$ wotj. a) $o$, b) (vor $l$ gewöhnlich) $a$.

Nach Wichmass gibt es für das Lautverhältnis syrj. $e \sim$ wotj. o etwa 40 Beispiele (Wotj. Vok. 76). Im Ostpermjakischen erscheint regelmässig ein durch $\ddot{o}$ wiedergegebener Vokal, z.B. syrj. ektini 'sammeln', PO óktti-, wotj. oktịn $\hat{\imath}$; syrj. gerd 'rot', PO görd, wotj. gord. Diesen $\ddot{o}$-Laut bildet man nach Genetz "mit der Lippenartikulation eines ziemlich engen $o$ 
und mit zurückgezogener Zunge, etwa zwischen $\theta[=$ "Gutturalvokal»] und e» (a.a.O. S. 4). Das Genetzsche $\ddot{o}$ ist somit entweder ein zwischen $o$ und $\ddot{o}$ stehender Mittelvokal $\dot{o}$ oder ein hinteres $\ddot{o}(\ddot{a})$.

Bubrich ist bei der Betrachtung der syrjänischen und wotjakischen Vokalverhältnisse zu dem Ergebnis gelangt, dass das im Wotjakischen als Entsprechung des syrj. e auftretende $o$ eine verhältnismässig späte Lautbildung sein müsse, deren Vorstufe im Altwotjakischen vielleicht vein vorderer, labialisierter, an einen Mittelvokal anklingender Vokal" gewesen sei (Боллетень ЛОИКФУН 4, 1929). Nach der Ansicht von Sternitz ist Bubrichs Gedanke unwahrscheinlich, obwohl auch er zugibt, dass das wotj. o recht jung ist, weil urperm. o im Wotjakischen $\mathrm{zu} u$ geworden ist (Vok. 128). Keiner von beiden Forschern hat beachtet, dass im Ostpermjakischen gerade der von Bubrich angenommene Laut, ein palataler. an einen Mittelvokal anklingender Labialvokal, sich erhalten hat. Es besteht kein Zweifel, dass er besser als die übrigen Vertretungsweisen die urperm. Qualität bewahrt hat. Da es im Urpermischen eine besondere Mittelvokalreihe gegeben hat, muss $\dot{o}$ als die ursprüngliche Form des zu dieser Reihe gehörenden Labialvokals angesehen werden und auch das $\ddot{o}$ des von Genetz erforschten Dialektes von Paršakova dürfte diesen Laut bezeichnen. Vgl. Verf., Virittäjä 1951 S. 444 -445. Auch Lytkis hat bei dieser Frage seine Aufmerksamkeit auf die Lautvertretung der ostpermjakischen Mundart gerichtet: "Es ist sehr möglich, dass in der altpermischen Sprache, wie auch im heutigen Komi-Jazvinischen, $\ddot{o}$ labialisiert war", Древнепермский язык 88. In den Известия Академп Наук СССР, Отцеление литературы и языка 1952 , S. 481 sagt er noch deutlicher, dass sich im KomiJazvinischen labialisierte Mittelvokale (ij, ö) erhalten haben, die in den komi-syrjänischen und komi-permjakischen Dialekten delabialisiert worden sind.

Wir können somit als allgemeine Lautvertretung vermerken: urperm. $\dot{\delta} \equiv$ in $\mathrm{PO}$, aber im Syrjänischen im allgemeinen durch Delabialisation $>e$, im Wotjakischen durch Velarisierung $>0$. 
Wichmann erwähnt fünf Fälle, in denen syrj. $e$ im Wotjakischen $a$ entspricht (a.a.O. S. 76). In Wirklichkeit ist ihre Zahl etwas grösser, nämlich 10-11. Wir zählen sie hier auf: syrj. elni 'aufhalten, hemmen usw.', PO ól-, wotj. alịnị; syrjP eve.dz' 'Schoss des Kleides', PO ốli $\tilde{J}$, wotj. al; syrj. kẹl 'Schnur, Strick', PO köl, wotj. kal; syrj. kętni 'mit dem Zugnetz fischen', wotj. kaltịn len', wotj. malpàni; syrj. pel 'Seite', PO pöl, wotj. pal; ? syrj. pelsalni 'schälen', PO pýlsal-, wotj. palan?̣ (möglicherweise gehört dieses Wort nicht zu dieser Gruppe, denn die Lautvertretung in der $\mathrm{PO}$-Mundart setzt ein urperm. ${ }^{*} \dot{q}$ voraus; es handelt sich also um einen Reihenübergang entweder im Ostpermjakischen oder im Wotjakischen); syrj. śela (in PK sporadisch $e>e$ ) 'Haselhuhn', PO sóla, wotj. śala; syrj. ślalni 'spucken, speien', PO sólal-, wotj. salanị; syrj. vęl 'Pferd', PO völ, wotj. val; syrj. vęlni 'sein', PO völ-, wotj. val 'war' (aber $i$ in vilini Inf.). - Wie die Beispiele zeigen, hat sich der Vokal der ersten Silbe im Wotjakischen vor $l \mathrm{zu}$ a gewandelt, vgl. Steisitz Vok. 127. Nicht immer aber finlen wir den Lautwandel $\dot{o}>a$ in dieser Stellung. Mindestens die folgenden Beispiele weisen im Wotjakischen auch vor $l$ die übliche $o$-Vertretung auf: syrj. delalni 'glänzen', wotj. dolanị; syrj. *keld: I kệd-nán 'kleiner, runder Kuchen', P keq.da•vni 'kneten', PO kóldal-, wotj. koldi 'eine Art Pastete'; ? syrj. pel 'Mal', PO pyl, wotj. pol (PO weist auf urperm. *' hin); syrj. tel 'Winter', PO töl, wotj. tol; syrj. telis 'Mond', PO tốliś, wotj. toleź. Von diesen Beispielen können wir wotj. pol unberücksichtigt lassen, denn ausser dem Umstand, dass die urperm. Grundform dieses Wortes unsicher ist, ist im Wotjakischen offenbar das Bestreben wirksam gewesen, eine störende Homonymie zu eliminieren: Im Urpermischen hat es vier Stämme *pól, *pól gegeben (mit den Bedeutungen 'Seite', 'Mal', 'Brett', 'das Innere'), die alle im Syrjänischen im allgemeinen die Form pel angenommen haben, im Wotjakischen aber - zum Teil sicherlich durch unregelmässige Lautentwicklung - voneinander geschieden worden sind: pal, pol, pul, pel- $(\sim$ pol- $)$. Definieren wir den Lautwandel $\dot{o}>$ wotj. 
$a$ in der Weise, dass er vor $l$ stattgefunden hat, ausser nach nichtmouillierten Dentalkonsonanten, so gibt es eine einzige Ausnahme von dieser Regel, nämlich wotj. koldi, wo wir die Form *laaldi annehmen möchten. Diese Ausnahme dürfte die Regel nicht aufheben. Es bestehen zwei Möglichkeiten: entweder $\dot{o}$ direkt $>a$ oder $\dot{o}>{ }^{*} o>a$. Die erste Alternative lässt sich phonetisch leichter erklären: Das $l$ hätte gleichzeitig velarisierend und den Artikulationsgrad öffnend gewirkt; lediglich nichtmouillierte Dentalkonsonanten, die dem Vokal vorhergingen, hätten vermocht, den velarisierenden Einfluss des $l$ auszuschalten.

Zählen wir die wotj. a)- und b)-Fälle zusammen, so erhalten wir insgesamt etwa 50 Beispiele für urperm. $\dot{o}$.

Über die Geschichte des urperm. $\dot{o}$ lässt sich Folgendes feststellen:

a) $\dot{o}<$ vorperm. ${ }^{*} \ddot{a}$ : jez $(243$; in syrjL sporadisch $e>e)$, kesjini (250), ? remiś (256), ? pek (262), reped (264), tel (271), pel (282), eve $d d^{\prime} z$ (290). Wir haben also 6-8 Beispiele.

b) $\dot{o}<$ vorperm. * $\ddot{u}$ : śelem $(420$; im Wotjakischen unregelmässig ${ }^{*} \dot{o}>>u$ ), śelaln $i$ (423), et- (433), šert (442). - Die Beispiele scheinen zumeist sporadische Fälle von Offenwerden des vorperm. ${ }^{*} \ddot{u}$ vor Liquida zu sein. (Im übrigen ist vorperm. ${ }^{*} \ddot{u}$ recht regelmässig zu urperm. $i$ geworden, s. SS. $300-301$.)

c) $\dot{o}<$ vorperm. ${ }^{*} a$; penni (33). - Dieser Fall von Vertretung des vorperm. ${ }^{*} a$ durch $\dot{o}$ ist ganz alleinstehend. Das Beispielwort ist stark affektiv, wodurch die Unregelmässigkeit seiner lautlichen Entwicklung sehr wohl verursacht sein kann.

d) $\dot{o}<$ vorperm. ${ }^{*}$ : veln $i$ (110; im Wotjakischen paradigmatischer Vokalwechsel $a \sim i$ ). - Hier handelt es sich um einen Fall von unregelmässiger Palatalisierung im Urpermischen.

e) $\dot{o}<$ vorperm. ${ }^{*} u$ : tedni (208). - Auch hier zeigt sich eine merkwürdige Unregelmässigkeit. Als Entsprechung von fi. tuntea würde seinem Lautbestand nach viel besser tidalni 'sichtbar sein, scheinen' passen. (Vorperm. * $u$ ist im allgemeinen sehr regelmässig zu urperm. i geworden, s. S. 300.) 
Mit Ausnahme von Punkt a) macht die Entwicklungsgeschichte des urperm. $\dot{o}$ einen sehr uneinheitlichen und von Zufällen bestimmten Eindruck.

6. Syrj. $e$ (im Anlaut und nach $k$ mundartlich ${ }^{*} o$ ), PO $u \sim$ wotj. $e(>i)$.

Nach Wichmanx (Wotj. Vok. 82) gibt es etwa 40 Beispiele für das Lautverhältnis syrj. e wotj. e. (Diese Zahl ist vielleicht etwas zu hoch gegriffen.) Der PO-Dialekt hat in den Wörtern, die diese Lautvertretung aufweisen, regelmässig den von Gexetz mit $y$ wiedergegebenen Vokal, z.B. syrj. lem 'Traubenkirsche', PO lym, wotj. lem; syrj. pedni 'ersticken'. PO pýd-, wotj. pedinî́; syrj. tel 'Wind', PO tyl, wotj. tel.

Die $y$-Vertretung in der PO-Mundart deutet darauf hin, dass in wenigstens sieben der Wörter, die das unregelmässige Lautverhältnis syrj. $e \sim$ wotj. $i$ aufweisen (nach Wichmann insgesamt 17 Fälle, s. a.a.O. 75), derselbe urperm. Ausgangslaut zugrundeliegt wie in der vorigen Gruppe, mit anderen Worten, im Wotjakischen hat sich in diesen Wörtern ein später, sporadischer Lautwandel $e>i$ vollzogen. Einige Beispiele: syrj. berjini 'wählen', PO býrii-, wotj. bị̂rjîn lez 'blau', PO lyz, wotj. l⿳⺈ $z$; syrj. ver 'Wald', PO vyr, wotj. $v \hat{\imath} r$.

Als dritte Gruppe kann man in diesen Zusammenhang die später im einzelnen zu behandelnden Fälle stellen - insgesamt sieben -, in denen der Vokal der ersten Silbe im Anlaut oder nach einem $k$ gestanden hat.

Alles in allem umfasst der in Rede stehende Fall etwa 50 gemeinpermische Wörter.

Von was für einem urperm. Vokal haben wir hier auszugehen? Unter den früheren Forschern herrschte allgemein die Auffassung, dass der sowohl im Syrjänischøn als auch im Wotjakischen anzutreffende illabiale Mittelvokal e eine Lautvertretung widerspiegle, die es bereits in der permischen Grundsprache gegeben habe. Im Ostpermjakischen aber sehen wir als Entsprechung des syrj.-wotj. e einen ganz andersartigen Vokal. Das Genetzsche $y$ hat man oft als $\underline{i}$ transkri- 
biert, anscheinend aus dem Grunde, weil bei Wiedemann $y$ das Zeichen für $i$ ist. Nach Genetz bildet man das $y$ "mit der Lippenartikulation des $u$ und der Zungenstellung zwischen $u$ und $i$ (a.a.O. S.4); es handelt sich also deutlich um den Mittelrokal $u$.

Das $2 u$ der PO-IIundart haben wir schon oben als palatalisierte Variante von $u\left(<\right.$ urperm. ${ }^{*} o$ und $\left.u\right)$ angetroffen, die ror $j$ oder mouilliertem Konsonanten auftritt, s. SS. $27 \pi$, 285. Wenn $u$ aber syrj.-wotj. e entspricht, ist es von den umgebenden Konsonanten unabhängig.

Eine wichtige Rolle bei der Lösung des $u$-Problems spielen die Dru c krerhält $\mathrm{n}$ is se. Nach der im Ostpermjakischen herrschenden, praktisch ohne Ausnahme geltenden Akzentregel ist in mehrsilbigen Wörtern der Druck auf derjenigen Silbe, in der sich der erste Vokal befindet, der einen urperm. nichtengen Tokal vertritt. Hat das Wort ausschliesslich solche Vokale, die urperm. enge Vokale vertreten, so liegt der Druck auf der letzten Silbe. (Vgl. Vir. 1951, S. 447. Lүтктх gibt in Труды I, SS. 90-99 eine ausführliche Darlegung der Druckverhältnisse im Ostpermjakischen.) - Diese Regrel bedeutet, dass in der ersten silbe nur diejenigen Vokale unbetont sind, die urperm. $u, i$ und $i$ fortsetzen, dass aber nicht bloss die Vokale, die offener als die engen Vokale geblieben sind, d.h. $a, o, \dot{o}$ und $e$, betont sind, sondern auch die, die erst sekundär eng geworden sind. Z.B. PO úkial- 'küssen' (syrj. okal-, SO okal-; urperm. o) lukál- 'mit den Hörnern stossen' (syrj. lukal-; urperm. u); midal- 'mieten, dingen' (syrj. medal-; urperm. ${ }^{*}$ ) $\sim$ kizáal- 'zuknöpfen' (syrj. kiźal-; urperm. i). Auch bei $u$ bemerken wir, dass es sowohl betont wie unbetont rorkommt. Betont ist ausser dem $u$, das urperm. o vertritt, auch das $u$, das syrj.-wotj. e entspricht, während aus urperm. $u$ durch Palatalisation entstandenes $u$ unbetont ist, z.B. šýćci- 'ruhen' (syrj. šoint'śi-, šot'sí-, SO šottš

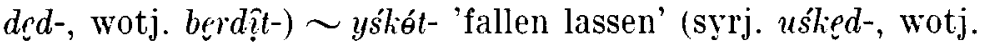
uślitt-; urperm. u). Im Lichte der Druckverhältnisse kann gar kein Zweifel darüber bestehen, dass PO $u$, wenn es syrj.wotj. e entspricht, auf einen Vokal zurückgeht, der offener 10 - Finnisch-ugrische Forschungen 
als ein enger Vokal war. Es wäre ganz natürlich, $u$ auch als Ergebnis einer Entwicklung anzunehmen, die parallel zu den Lautentwicklungen $u<o$ und $i<*_{e}^{*}$ verlaufen sein dürfte (vom Lautwandel $i<{ }^{*} e$ wird auf S. 310 die Rede sein). Dann gelangen wir zum Entwicklungsgang $u<{ }^{*} \dot{a}$, mit anderen Worten, die Ausgangsform wäre ein Laut gewesen, der mit dem oben im $\tilde{o}$. Kapitel behandelten $\dot{o}$ ein Mittelvokalpaar gebildet hätte, das den Paaren $0: 0$ und $e: \ell$ entsprochen hätte. Vgl. Verf., Vir. 1951, S. 447. Auch Lүткгх, Tруды I, ऽ. 80, hält es für "sehr möglich", dass es im Altsyrjänischen zwei $\ddot{o}$, ein offenes und ein geschlossenes, als Entsprechungen von $\ddot{o}$ und $\ddot{y}$ des Ostpermjakischen gegeben hat.

Die einheitlichen und logischen Entwicklungslinien, die sich so vom PO-Dialekt aus eröffnen, scheinen zu beweisen, dass sich diese Mundart in einer ähnlichen Schlüsselstellung hinsichtlich der Erhellung des Ursprungs des in Rede stehenden Vokals befindet wie hinsichtlich des urperm. $\dot{o}$. Vielleicht aber wird man einwenden: Der labiale Charakter des altostpermjak. ${ }^{*} \dot{o}$ und sein im Vergleich zu der Normalaussprache des syrj.-wotj. e doch wohl geschlossener Artikulationsgrad können doch auf einer Sonderentwicklung in diesem Dialekt beruhen. Eine endgültige Antwort auf die Frage: ist urperm. ${ }^{*} \dot{0}>$ syrj.-wotj. $e$ oder umgekehrt urperm. ${ }^{*} e>$ PO ${ }^{*} \dot{q} ?$ ? müssen wir somit woanders suchen. Aufschluss gibt uns die folgende fünf Wörter umfassende Wortgruppe, für die ein im Anlaut sekundär entwickeltes $v$ kennzeichnend ist, das in den syrj. Mundarten weithin vorkommt, z.B. syrj. (im allgemeinen) vom, Udora $v e m$, SO om, P em 'Mund, Öffnung', PO $y m$, wotj. im, (Musk.) em; syrj. von, U ven, SO òn, P en 'Bettrorhang', PO yn, wotj. in; syrj. voš-, U vešs-, SO oš-, P ess- 'verloren gehen, umkommen', PO ýš-, wotj. išs; syrj. vos, U ves, SO os, $\mathrm{P}$ es 'Erbrochenes, das Erbrechen', PO ys-, wotj. esk-; syrj. vor, U ver, SO or, P er 'Trog', PO yr. Uotila nimmt an (PK 62), dass der ursprüngliche Laut in diesen Wörtern ein an einen Mittelvokal anklingender Labialvokal, vielleicht ${ }^{*} g$, gewesen ist, vor dem sich im Syrjänischen ein $v$ herausgebildet hat, während der Vokal selbst zu o oder aber zu $e$ geworden ist. Das ist ganz richtig erschlossen, und wenn man 
der von Uotila rekonstruierten Grundform noch das Kennzeichen der Geschlossenheit hinzufügt, das gerade die Vertretung in der konservativen SO-Mundart erfordert, so kommt man praktisch bereits zum Vokal $\dot{\rho}$. Also beweist das im Syrjänischen vor den Vokal getretene $v$ unwiderlegbar, dass der (lem $e$ vorhergegangene Vokal in der Zeit der Gemeinsamkeit der westsyrjänischen Dialekte wenigstens im Anlaut ein Labialvokal gewesen ist.

Auch für einen urperm. labialen ${ }^{*} \dot{g}$-Laut nach $k$ scheint sich ein offensichtlicher Beweis erhalten zu haben. Es gibt zwei Beispielwörter, die im ganzen permischen Sprachgebiet verbreitet sind: syrj. (im allgemeinen) kos, SO kos, $\mathrm{P}$ kes 'trocken', PO kys, wotj. kes; syrj. koz, SO kòz, P kez 'Fichte', PO kyz, wotj. kîz. In diesen Wörtern sind die Vokalverhältnisse der verschiedenen Mundarten die gleichen wie bei den im vorigen Kapitel besprochenen Wörtern (ein Unterschied ist nur der, dass nach $k$ auch in der Mundart ron Udora wie in den übrigen westlichen Dialekten o erscheint: kos, koz), so dass auch hier das Problem seiner natürlichen Lösung zugeführt wird, wenn man von ursprünglichen Formen mit $\dot{g}$ alusgeht.

Wir erinnern uns, dass vor urperm. $o$ in vielen Wörtern sich ein sekundäres $u$ herausgebildet hat, wenn der Vokal im Anlaut stand oder auf $k$ folgte. Unter ganz gleichen Voraussetzungen hat eine Sonderentwicklıng auch bei dem entsprechenden Mittelrokal stattgefunden. Wahrscheinlich ging anfangs eine Diphthongierung vor sich: ${ }^{*} \dot{a}$ bzw. $\bar{g}>{ }^{*} u \dot{a}$. Danach dürfte sich das im Anlaut stehende ü der westsyrjänischen Mundarten nach bekannter Weise in $v$ verwandelt haben, während das $* \dot{a}$ in allen diesen Dialekten ausser dem von Udora zu $o$ velarisiert wurde; im letztgenannten Dialekt hingegen blieb es ein Mittelvokal und wurde zu $e$ delabialisiert. In der SO-Mundart verschwand das $v$ später ganz ebenso wie bei der Entwicklung ror urperm. o: $*^{*} u>{ }^{*} v>0$. Auch nach $k$ können wir uns gut die Entwicklung ${ }^{*} k \dot{\alpha}-$ bzw. ${ }^{*} k \bar{o}->$ ${ }^{*} k u \dot{o}^{-}>{ }^{*} k u_{0}->k o-$ vorstellen $\left(\mathrm{vgl} .{ }^{*} k o->{ }^{*} k u o->k o-\right.$ in denselben Mundarten). Aber sind die Entwicklungen urperm. ${ }^{*} 0>{ }^{*} u_{n}$ und urperm. ${ }^{*} \dot{a}>{ }^{*} u_{\alpha} \dot{\alpha}$ gleichzeitig vor sich gegan- 
gen? Die genaue U̇bereinstimmung der Voraussetzungen für beide und ihre gleichmässige Durchführung in den westsyrjänischen Dialekten scheinen eindringlich eine bejahende Antwort auf diese Frage nahezulegen. Auch die permjakischen Mundarten, sowohl das West- wie das Ostpermjakische, stehen dieser Möglichkeit nicht entgegen, denn in ihnen kann man die völlig übereinstimmenden Lautentwicklungen $*_{0}>$

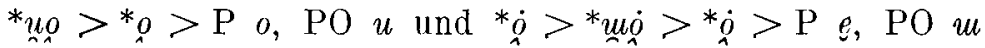
annehmen. Die sekundären halbvokalischen Vorschläge $u$ und $u$ wären also dort verschwunden, ohne irgendeine Spur zu hinterlassen. Lediglich im wotjakischen Gebiet hätte sich die Entwicklung des *uo von der des *uo scharf unterschieden; $u$ hat sich erhalten, aber * $u$ wäre ebenso behandelt worden wie in den permjakischen Dialekten. Das ist ja sehr gut möglich, zumal wenn man in Betracht zieht, in wie grundverschiedene Richtungen auch die Vokale $o$ und ${ }^{*} \dot{q}$ selbst sich in diesen Fällen im Wotjakischen entwickelt haben.

Es ist klar, dass das von uns im Anlaut und nach $k$ festgestellte urperm. ${ }^{*} \dot{a}$ nicht von Anfang an unter so begrenzten Voraussetzungen hat auftreten können. Somit muss die Vertretung im Ostpermjakischen, wo auf urperm. ${ }^{*} \dot{0}$ zurückgehendes $u$ in allen Stellungen als ausschliessliche Entsprechung von syrj.-wotj. e erscheint, den ursprünglichen Stand widerspiegeln, mit anderen Worten, sie zeigt, dass dieses syrj.-wotj. $e$ auf dem Wege der Delabialisation entstanden ist. Die Delabialisierung des $* d$ hat vielleicht schon in irgendwelchen späturpermischen Mundarten begonnen, so dass die allgemeine $\bullet$-Vertretung im Syrjänischen und Wotjakischen zum Teil eine gemeinsame Grundlage haben kann.

Über die Entwicklungsgeschichte des urperm. ${ }^{*} \dot{o}$ (mit dem zusammen wir auch eventuelle urperm. *uó - Fälle behandeln) können wir Folgendes feststellen:

a) ${ }^{*} \dot{o}<$ vorperm. ${ }^{*} \bar{o}$ : kez $(156$; dies Wort geht ganz unstreitig auf eine Form mit ${ }^{*} \bar{o}$ zurück, ebenso wie lp. guossâ), né (157; syrjS VL sporadisch $i$ ), sem (162), sen (163; wotjU sporadisch i), lem (164), velalni (165; mundartlich im Syrjänischen und sporadisch in $\left.\mathrm{PO}{ }^{*} v_{\mathbf{o}^{-}}>v 0^{-}\right)$, ver $(166$; im Wotjakischen $e>i)$, ? demni (168), ? seshini (169; im Wotjaki- 
schen $e>i$ ). Insgesamt haben wir also $7-9$ Beispiele. Vom phonetischen Standpunkt aus gesehen äusserst natürlich wäre z.B. die Entwicklungsreihe $*_{\bar{o}}>*_{\bar{o}}>*_{\bar{o}}>*_{\dot{o}}$ : Der Artikulationsgrad des langen Vokals wäre gegenüber dem ursprünglichen Zustand ein wenig geschlossener geworden und hätte sich zugleich in palataler Richtung weiterentwickelt. Für derartige Lautentwicklungen könnte man aus verschiedenen Sprachen Beispiele anführen. Es ist zu beachten, dass man sich die oben rekonstruierte Zwischenstufe $*^{*} \dot{a}$ als auf rein permischem Boden herausgebildet denken muss. Durch sie bekommen wir schemenartig die urperm. langen Vokale ins Blickfeld, von denen sich direkte Spuren in keiner perm. Sprache oder Mundart erhalten haben, die es aber doch gegeben haben muss.

b) ${ }^{*} \dot{d}<$ vorperm. ${ }^{*} \boldsymbol{o}$. Auch einige derartige Fälle gibt es. Interessant ist die Feststellung, dass man die an sich ganz vereinzelt dastehende Lautentwicklung $*_{0}>>*_{0} \dot{0}$ in ein paar Wörtern als Folge einer sekundären Vokaldehnung ansehen kann, die durch Verschmelzung des unmittelbar auf den Vokal folgenden Konsonanten mit diesem hervorgerufen worden sein dürfte: vosni (109; liesse sich die Entwicklungsreihe

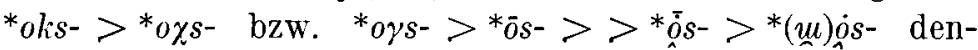
ken? Diese Erklärung würde roraussetzen, dass das im Wotjakischen statt $s$ im Inlaut mundartlich auftretende -sksekundär wäre und nicht auf einer Metathese von ursprünglichem -lis- beruhen würde.), kos (149; auch hier möchte man in erster Linie an eine Lautentwicklung *koks- $>{ }^{*} k o \mathrm{zs}^{-}$

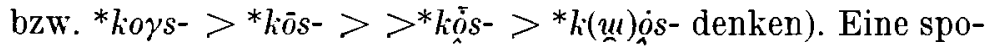
radische Palatalisierung von kurzem $*_{o}$ vor $i$ dürfte vorliegen in wotj. vej $\left(129\right.$; im Syrjänischen wird das $*_{a} \dot{a}$ von sporadischem $i$, ja sogar $i$ fortgesetzt, was ebenfalls dem assimilierenden Einfluss des darauffolgenden $i$ zuzuschreiben ist).

c) ${ }^{*} \dot{a}<$ vorperm. ${ }^{*} o$ oder ${ }^{*} a$ : be $e^{\frac{z}{z}}$ (83; im Wotjakischen sporadisch $e>i$ ). Auch hier ist es nicht leicht, auf eine andere Weise zu urperm. ${ }^{*} \dot{o}$ zu kommen als durch Annahme einer Dehnung des Vokals (? frühurperm. ${ }^{*} o$ ).

d) ${ }^{*} \dot{i}<$ vorperm. ${ }^{*} \bar{u}$ : tel (237). Die lautgesetzliche Form wäre ${ }^{*} t i l$, weil vorperm. ${ }^{*} \bar{u}>$ im allgemeinen $\underline{i}$ (s. S. 302). 
Es handelt sich deutlich um einen gelegentlichen Übergang in die ${ }^{*} \dot{o}$-Reihe zwecks Vermeidung von Homonymie; danach blieben in der Sprache immer noch drei verschiedene Wörter übrig, die til lauten, nämlich in der Bedeutung 'Zugnetz', 'Schwungfeder' und 'Feuer'.

Indem wir einige etymologisch oder hinsichtlich ihrer Lautentwicklung unklare $* \dot{i}$-Wörter übergehen, wollen wir schliesslich noch den allgemeinen Eindruck feststellen, dass dieser Vokal fast regelmässig Fortsetzung eines vorpermischen oder im Urpermischen entwickelten langen $o$-Lautes zu sein scheint. Auch von diesem Gesichtspunkt ist es sehr natürlich, dass das urperm. ${ }^{*} \dot{\rho}$ ein Labialvokal war. Man hat zuweilen auf Grund der Tatsache, dass das von altersher als lautgesetzliche Entsprechung des ostseefi. $\bar{o}$ erkannte syrj.-wotj. $e$ illabial ist, irgendeinen uralten "Ablautwechsel" zwischen labialem und illabialem Vokal behauptet. Derartige Ansichten entbehren also jeglicher Grundlage.

Anhang zu den Kapiteln über. urperm. $\dot{o}$ und *ón.

Es gibt ein paar Wörter, bei denen sich nicht sicher sagen lässt, ob in ihnen ein urperm. $\dot{o}$ oder $* \dot{o}$ gestanden hat.

a) Auf vorperm. ${ }^{*} a$ geht der Vokal der ersten Silbe in veini (50) zurück, das insofern merkwürdig ist, als im Ostpermjakischen in diesem Wort ein Vokalwechsel $u \sim \dot{o}$ auftritt: $v y^{\prime} j-\sim v o ̂ ́ t ' ;$ ferner ist das $\underline{i}$ in der wotj. Entsprechung unregelmässig, einerlei ob man von $\dot{o}$ oder von $*_{\dot{a}}$ auszugehen hat. Die Entwicklung des ursprünglichen ${ }^{*} a$ zu einem Mittelvokal muss in diesem Wort auf der palatalisierenden Einwirkung des darauffolgenden $i$ beruhen. Aber in welchem Stadium ist diese sporadische Palatalisierung erfolgt? Es gäbe zwei Möglichkeiten: ${ }^{*} v a i->{ }^{*}$ väi- $>{ }^{*} v o i-$ usw. oder: ${ }^{*} v a i->>{ }^{*} v o i-$ $>{ }^{*} v o i-$ usw. Welche dieser beiden angenommenen Entwicklungsreihen den wirklichen Verlauf der Lautveränderungen besser wiedergibt, dürfte sich unmöglich mehr klarstellen lassen. Vgl. auch S. 318.

b) Von rorperm. *o ist in rèž (134) auszugehen. Da für 
dieses Wort keine Entsprechungen aus dem Ostpermjakischen und auch nicht aus dem Wotjakischen aufgezeichnet worden sind, könnte man sagen, dass es sich hier sogar um urperm. ${ }^{*} \varepsilon$ handeln kann (s. SS. 303-305), aber es erscheint doch unmöglich, sich eine Lautentwicklung $*_{o}>>{ }^{*} \varepsilon$ vorzustellen. Am wahrscheinlichsten kommt einem die Entwicklung $*_{0}>>*_{0}$ $>^{*} \dot{q}$ vor, deren letztes Stadium das Ergebnis einer sporadischen Palatalisierung wäre, die das anlautende $\dot{n}$ hervorgerufen hätte.

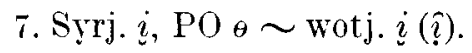

Nach Wrohmanx (Wotj. Vok. 82) gibt es etwa 110 Wörter. die sowohl im Syrjänischen als auch im Wotjakischen $i$ haben, eine Zahl, die offenbar recht genau angegeben ist. Im Ostpermjakischen weisen diese Wörter regelmässig einen Laut auf, der von dem geschlossenen Mittelvokal des Syrjänischen und Wotjakischen stark abweicht. Gexerz definiert diesen von ihm mit $\theta$ bezeichneten Laut wein ziemlich offener, dem a etwas ähnlicher Gutturalvokal ohne Lippenartikulation" (a.a.O. S. 4). LYTkis, der gleichfalls Gelegenheit gehabt hat, mit eigenen Ohren diesen Vokal zu hören, nennt ihn einen hinteren Vokal der unteren Reihe, der ans russ. a erinnert, aber um einiges enger und mehr hinten ist. Akustisch kommt das $\theta$ dem vortonigen $a(\Lambda)$ der Leningrader Gegend in den Wörtern водa, сама (вАда́, снмá) recht nahe, s. Древнепермский язык 92. In der ersten Silbe mehrsilbiger Wörter ist $\mathrm{PO} \theta$ unbetont; das ist einer der klarsten Beweise dafür, dass dieser Vokal auf einen geschlossenen Vokal zurückgeht, d.h. auf das im Syrjänischen und Wotjakischen erhalten gebliebene $i$. Der Lautwandel urperm. $i>\mathrm{PO}_{\theta}$ dürfte mit der in den nichtersten Silben ror sich gegangenen Ausgleichung zusammenhängen, durch die $\theta$ zur Fortsetzung nicht nur von $i$, sondern auch eines offeneren Vokals (= syrj. e) geworden ist. In einigen wotjakischen Dialekten ist $i$ regelmässig reduziert und häufig ist auch der durch mouillierte Konsonanten und $j$ verursachte Lautwandel $i>i$ anzutreffen, s. Wichmann, a.a.O. SS. VI, 19-22. 
a) $i<$ vorperm. ${ }^{*} u$ : $\check{s} i$ (171; sowohl im Syrjänischen wie im Wotjakischen mundartlich sporadischer Lautwandel $i>i$ ), ki- (174), kilalni (176), kim (177), kimni (178), wotj. kidź (181; im Syrjänischen sporadisch $k i->k u-)$, wotj. kịs?̣n? (180; im Syrjänischen wieder ki- $>k u-)$, kini (183; im Wotjakischen sporadisch $i>u)$, lid (185), lím (186), li (188), míśkin $\underline{\imath}$ (189; im Wotjakischen $i>i$ unter dem Einfluss des darauffolgenden $s$; dieses perm. Wort geht wie seine tscher. Entsprechung deutlich auf eine Urform mit $u$ zurück), bitškini (194), pinni (196; im WotjG sporadisch $i>u$ ), piridzz (197), pira (198; im Wotjakischen $i>u$ ), pidzírtni (200), sil (202), till 'Feuer' (205), tirp (209), syrjI $i$ (212), ? vil (215; verschiedentlich sporadischer Lautwandel $i>i$ vor $l$; bei diesem Wort könnte man auch von ${ }^{*} \bar{u}$ ausgehen, wenn es möglich wäre, sich vorzustellen, dass das ostseefi. $\bar{u}$ älteren Ursprungs und nicht nur ein Ergebnis einer unregelmässigen Vokaldehnung im Urfinnischen wäre), tịl 'Schwungfeder' (216), kizni (217), wotj. kị̂nîn (219; im Syrjänischen sporadisch $k \underline{i}$ - $>k u$-), wotj. mixig (221), wotj. iskal (224), miz (225), nịl (226), pides (227), pirni (228), piž (229), tik (230). Insgesamt sind das 32-33 Beispiele, also eine aussergewöhnlich grosse Zahl, wodurch bewiesen wird, wie regelmässig die Lautentwicklung $*_{u}>i$ gewesen ist.

b) $i<$ vorperm. * $\ddot{u}$ : $\operatorname{kin}$ (408), giz (409; im Wotjakischen mundartlich $i>i$ ), wotj. kitkini (412), wotj. kii (413), wotj. kil $i$ (414; mundartlich $i>i$ vor $l^{\prime}$ ), gird'źa (415), mid wotj. pîzñi (419; mundartlich $i>i)$, sil (422; wotj. mundartlich $i>u$ ), syrjP $\operatorname{din}$, wotjG $\operatorname{din}$ (430; sonst im Syrjänischen und Wotjakischen $i>i$, vgl. Wichmaxi Wotj. Vok. 19, UotrLA PK 251), vil (434), hiz (438), lis (439), lisstini (440), mik (441). Insgesamt haben wir hier 15 Beispiele.

Urperm. $\underset{i}{i}$ ist die lautgesetzliche und beinahe ausschliessliche Fortsetzung von vorperm. * $u$ (vgl. Gombocz NyK XXXIX 274) und ausserdem auch von vorperm. * $\ddot{u}$. Man muss annehmen, dass ursprüngliches ${ }^{*} u$ und $*_{\ddot{u}}$ vor ihrer Delabialisierung zusammengefallen sind. Nach der Delabialisierung wäre nämlich eine Verschmelzung dieser Vokale nicht möglich gewesen, weil dann $*_{\ddot{u}}$ mit ursprünglichem $*_{i}$ zusammen- 
gefallen wäre, das aber völlig anders vertreten ist als vorperm. ${ }^{*} u$. Zwar schreibt Steinitz (Vok. 21, 25), dass vorperm. $*_{u}$ (= Steinitz $*_{o}$ ) und $*^{*} \ddot{u}(=$ Steinitz $* \check{o})$ sich in den perm. Sprachen in verschiedene Richtungen entwickelt hätten, nämlich nur $*_{u}$ wäre zu $i$ geworden, $*_{\ddot{u}}$ dagegen im allgemeinen $\mathrm{zu} i$ und lediglich in einigen Sonderfällen zu $i$, wie neben $l$ und $s$. Die Stellungnahme von Steinitz ist schwer zu verstehen, denn erstens ist in allen $* \ddot{u}$-Wörtern, die einen interdialektalen Wechsel $i \sim i$ aufweisen, das $i$ deutlich durch sekundäre Palatalisation aus $i$ entstanden und zweitens gibt es alles in allem drei Wörter, bei denen wir in den perm. Sprachen als Entsprechung der Labialvokale der ostsee- und wolgafinnischen Sprachen ausschliesslich $i$ antreffen. In zweien von ihnen (kiśmini, is) darf man vermuten, dass das $i$ älter ist als der Labialvokal, den ihre fi.-wolg. Entsprechungen aufweisen, und in einem (wotj. siźîl) ist urperm. ${ }^{*} i>i$ möglich, s. S. 315. - Die Entwicklung ist also folgendermassen verlaufen: vorperm. ${ }^{*} u$, $* \ddot{u}>$ Labialvokal $\left(* u, * \ddot{u}\right.$ bzw. $\left.{ }^{*} u\right)>i$.

c) $i<$ vorperm. ${ }^{*} \bar{e}$ : li $i l$ (353), wotj. lim (355; syrj. liem ist mit syrj.-wotj. l'em 'Leim' verwechselt worden), mil (356), niln $\underline{i}$ (357), nìr (361). Insgesamt sind das fünf Beispiele, auf Grund deren man sagen kann, dass vorperm. ${ }^{*} \bar{e}$ v vor nichtmouilliertem Konsonanten ausnahmslos zu $i$ geworden ist. ( Für $*_{\bar{e}}$ vor mouilliertem Konsonanten gibt es ein einziges Beispiel: bel [359], das darauf hinweisen dürfte, dass $*_{\bar{e}}$ in dieser Stellung ein Vordervokal geblieben ist.) STEINITz stellt die Dinge verkehrt dar, wenn er erklärt, das $\underset{i}{i}$ dieser Wörter sei gar nicht die Fortsetzung von fi.-ugr. ${ }^{*} \bar{e}$ (= Steinitz "Vollvokal $e »)$, sondern eines "reduzierten $\check{e} »$, das angeblich in einem Ablautwechsel mit $*_{\bar{e}}$ stand (Vok. 50-51). In Wirklichkeit passt die Entwicklung des $*_{\bar{e}}$ ausgezeichnet dazu, was wir oben über die Entwicklung des vorperm. kurzen $*_{e}$ dargelegt haben. Auch dieses ist vor nichtmouilliertem Konsonanten velarisiert worden (zu urperm. o und o). Die Entwicklung des $*_{\bar{e}}$ zu einem geschlosseneren Vokal als ursprünglich hat sich wiederum parallel mit dem Lautwandel des zweiten halbengen langen Vokals, $*_{\bar{o}}$, zu $*_{\dot{o}} \dot{0}$ vollzogen. Eine Zwischenstufe in der Entwicklung $*_{\bar{e}}>\hat{i}$ hat sicherlich das frühur- 
perm. ${ }^{*} \bar{c}$ gebildet, das beim Schwinden der Quantitätsunterschiede mit dem unter a) und $b$ ) besprochenen ursprünglichen kurzen $i$ zusammenfiel.

d) $i<$ vorperm. ${ }^{*} \bar{u}$ : kilni (233), sinni (236), $i z ̌ z ~(238)$. Ausser diesen drei Beispielen sei auf wotj. jir (232) aufmerksam gemacht. Wenn dies Wort dem fi. juuri entspricht, kann es sehr wohl den ältesten Stand des Permischen widerspiegeln. aus dem syrj. jur durch einen sporadischen Lautwandel $i>u$ entstanden sein kann. Im Vorbeigehen wollen wir noch auf das in mehrfacher Beziehung dunkle Wort kun (239) zurückkommen, vgl. S. 195. Wenn es unmittelbar dem lp. gudnâ entspricht, möchte man eine Entwicklung ${ }^{*} k \bar{u}-$ $>$ urperm. ${ }^{*} k i->$ syrj. $k u$ - annehmen, wie sie sporadisch in vielen anderen Wörtern festzustellen ist. Aber tscher. kon deutet doch vielleicht eher auf eine Urform mit $*_{\bar{o}}$ hin. Der Lautwandel $*_{\bar{u}}>i$ ist erwartungsgemäss. Die Entwicklung ist folgendermassen verlaufen: ${ }^{*} \bar{u}>\left(?{ }^{*} \bar{u}>\right)^{*} \bar{\imath}>i$. Die Delabialisierung dürfte zur selben Zeit vor sich gegangen sein wie auch bei dem Vokal, der ursprüngliches kurzes ${ }^{*} u$ fortsetzte. Das letzte Entwicklungsstadium, die Kürzung der langen Vokale, führte eine völlige gegenseitige Angleichung der Vokale herbei, die ${ }^{*} \bar{u}$ und ${ }^{*} u$ vertraten.

Die obigen Punkte a-d) zeigen, auf welche vier Ausgangspunkte das urperm. $i$ normalerweise zurückgeht. Folgende Fälle sind sporadisch:

e) $i<$ Vordervokal $+* v$. In diesen Fällen dürfte sich anfangs ein geschmolzener Vokal $* \ddot{u}$ gebildet haben, der dann wie das ursprüngliche $* \ddot{u}$ zu $\underset{i}{i}$ geworden ist. Derartige Wörter sind $\sin \left(270 ;\right.$ im Wotjakischen $o$ ), $t i(275)$, in denen $*_{-a ̈} v->>$ $*_{\ddot{u}}>\underline{i}$; ferner lïni (314; mundartlich $\ddot{i i}>i$ ), śili (334; gewöhnlich sowohl im Syrjänischen als auch im Wotjakischen unter dem Einfluss des vorhergehenden $\dot{s} i>i)$; in den zwei letzten Wörtern ${ }^{*}-e v-\left(<*_{-e p-}\right.$ in sili $)>>*_{i}>i$.

f) Der in Punkt e) dargestellten Vokalschmelzung grundsätzlich nahe steht die Lautentwicklung $i<$ Hintervokal $+j(\underset{n}{i})$. Die Entwicklung -oj- $(o \underset{n}{i})>i(\underset{n}{i})$ wird sichtbar in den Wörtern kia (93), kir (94), vielleicht auch syrj.-wotj. pi, wotj. (WIED.) pöi (118; nach Uotila PK 265 ist die Ausgangs- 
form *pii möglich; es wäre eine Entwicklung $\underset{\sim}{i i}(i j)>i$ vor sich gegangen, über die näher s. a.a.O.S. 267). Bei diesen Wörtern handelt es sich um die Verschmelzung eines vorperm. Hintervokals mit $j(i)$. Bei nil (321) ist die Entwicklung im übrigen in ganz gleichen Bahnen verlaufen, aber der von der Verschmelzung betroffene Hintervokal geht auf den rorperm. Vordervokal ${ }^{*} e$ zurück; also ungefähr $*_{-e j-(e i)}^{*}>$ ? ${ }^{*} o j>$ $* i \underset{i}{i}>$.

g) $i<$ vorperm. ${ }^{*} i$ : šir (363), rinišs (383; im Wotjakischen kommt auch die vordervokalische Form inšir vor, die sekundär sein dürfte). Liesse es sich denken, dass in diesen Wörtern unter dem Einfluss des $r$ und des $s$ eine sporadische Labialisierung $*_{i}>*_{\ddot{u}}$ vor sich gegangen ist?

h) $i<$ vorperm. ${ }^{*} a$. Es gibt einige Wörter, bei denen vielleicht auch eine solche Lautentwicklung in Frage kommt: wotj. ibini (4), kik (17), wotj. tilis (44). Nun ist jedoch daran zu denken, dass erstens Zahlwörter oft lautliche Schwankungen aufweisen (man beachte namentlich im Syrjänischen die merkwürdige Doppelheit $k i k$ 'zwei' $\sim k e k j a ̀ \cdot m i s$ 'acht'), so dass es nicht sicher ist, ob kik unmittelbar mit fi. kaksi verglichen werden muss. Die beiden anderen Wörter kennt man lediglich im Wotjakischen, wo es eine gewöhnliche Erscheinung ist, dass $i$ sich sekundär anstelle von urperm. $u$ ausgebreitet hat. Vielleicht sind auch den Formen ibini, tilis lautgesetzliche frühere Formen *ubini, *tulis vorausgegangen; in dem letztgenannten Wort dürfte der Lautwandel $*_{u}>$ ? gerade deshalb stattgefunden haben, weil man Verwechslungen mit tulis 'Frühling' vermeiden wollte. Zu kirnįs (13) rgl. S. 198.

8. Syrj. $e, \mathrm{PO} \dot{o} \sim$ wotj. (im allgemeinen) $e,($ Mtrk. auch) $\ddot{a}(=\varepsilon),($ WIED, auch $) \ddot{o}(=e)$.

Das Lautverhältnis syrj. $e \sim$ wotj. $e$ treffen wir in etwa 40 Wörtern an, vgl. Wrchmans Wotj. Vok. 81. Im Ostpermjakischen weisen diese Wörter regelmässig $\dot{o}$ auf, was deutlich mit der Lautvertretung im Ursyrjänischen übereinstimmt. Hier kann man jedoch nicht wie im 5 . Kapitel bereits von 
einem urpermischen $\dot{o}$-Vokal ausgehen, denn im Wotjakischen finden wir diesmal eine wesentlich andere Lautvertretung. Während normalerweise urperm. $\dot{o}$ im Wotjakischen durch $o$ vertreten wird, sehen wir o gar nicht in dem jetzt in Rede stehenden Fall. Merkwürdig ist wotj. $e$ in einem seltenen Dialekttypus, über den sich bei WiedEMANx im wotjakischen Anhang seines syrjänischen Wörterbuches (Syrjänisch-deutsches Wörterbuch. Anhang enthaltend das Wotjakische; sS. 461-567) Angaben finden. In 27 darin enthaltenen Wörtern, die den Typus syrj. $e \sim$ wotj. $e$ vertreten, erscheint $17 \mathrm{mal} \ddot{\partial}(=e)$, zweimal sowohl $\ddot{o}$ wie $e$ und achtmal ausschliesslich $e$. Wichmann beispielsweise zweifelt nicht an der Richtigkeit dieser $\ddot{o}$-Vermerke, die von der normalen Lautvertretung des Wotjakischen stark abweichen, sondern erwähnt sie als Beispiele für einen interdialektalen Wechsel $e \sim e$ im Wotjakischen (a.a.O. S. 59-61). Auf demselben Standpunkt scheint JemelJavov zu stehen (Грамматика вотяцкого языка 20,30). Man kann die wichtige Feststellung machen, dass bei Wiedemann niemals in solchen Wörtern $\ddot{o}$ vorkommt, in denen wotj. $e$ syrj. $e$ entspricht und in denen man von einem schon urperm. $e$-Vokal ausgehen muss. Wir gelangen also zu dem Ergebnis, dass das soeben in Rede stehende Lautverhältnis auch nicht gut als Fortsetzung von urperm. ${ }^{*} e$ passt. Aufschluss geben dürften die wotjakischen Aufzeichungen von Munkácsi Er schreibt regelmässig im Anlaut und manchmal auch im Inlaut, namentlich ror Liquida und $j$, anstelle von $e \ddot{a}$, dessen Lautwert $\varepsilon$ ist, also ein Vokal zwischen $\ddot{a}$ und $e$. Dies $\varepsilon$ verdient ernsthafte Beachtung, denn da es im Wotjakischen in nichtersten Silben auftritt, ist es offenbar sehr alt. Es sei folgendes im Auslautvokalismus zutagetretende Lautverhältnis erwähnt, das z.B. für die Illativendung typisch ist: wotj. murte, syrj. (im allgemeinen) morte, Ižma morte 'in den Menschen'. Da in so weit voneinander entfernten Gebieten wie im wotjakischen Sprachgebiet und in dem der nordsyrj. Ižmamundart $\varepsilon$ vorkommt, muss dieses urpermisch sein. Als natürlich möchte einem der Gedanke erscheinen, dass dieser Laut auch zum Vokalismus der ersten Silbe 
gehört hätte und das in Rede stehende Lautverhältnis auf ihn zurückgeht. Das urperm. ${ }^{*} \varepsilon$ der ersten Silbe wäre also im Ursyrjänischen zu $\dot{o}$ labialisiert worden, das im PO-Dialekt $\equiv$, aber sonst $>e ;$ im Wotjakischen wäre ${ }^{*} \varepsilon$ mundartlich in bestimmten Stellungen $\equiv$, im allgemeinen jedoch $>e$, in irgendeinem seltenen Dialekttypus $>e$.

Es folgen einige Beispiele, die die Lautvertretung in den verschiedenen Mundarten zeigen: syrj. egir 'glühende Kohle', PO öger, wotj. ègị̂, (MUNk.) ägi̊r, (WIED.) ögyr; syrj. emid'ź 'Himbeere', wotj. emeź, (Munk.) ämez̆, ämeź, (WIED.) ömez; syrj. eztinni 'anzünden', PO ốzdit-, wotj. estîn $\hat{\imath}$, (MuNk.) äst-, (WIED.) estyny; syrj. gerr 'Pflug', PO gör, wotj. ger?̂, (Munk.) gäri, (WrEd.) göri; syrj. jektini 'tanzen', PO jökti-, wotj. eltịn̂,, (Munk.) ält-, (WiEd.) jöktyny, öltyny; syrj. terni 'Raum oder Platz haben oder finden, hineingehen', PO tö́r-, wotj. ter?̣̂n़, (MUNk.) tär-, (WIED.) töryny; syrj.zem 'aufrecht', wotj. zem, (MUNk.) zäm, (WIED.) zem 'wahr'. - Man beachte jedoch die Wortpaare syrjPO tel' 'lichtes, junges Gehölz von Nadelbäumen' $\sim$ wotj. Munk. täl' 'Wald' und syrj. t'śet'śsini 'aufspringen, aufstehen', PO céćci- $\sim$ wotj.

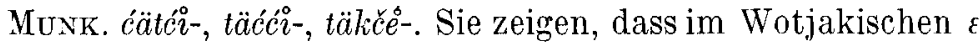
auch urperm. $*_{e}$ vertreten kann. Trotzdem fällt es schwer, das Lautverhältnis wotj. $\varepsilon \sim$ syrj. $e$ in der ersten Silbe vom entsprechenden Lautverhältnis in den nichtersten Silben zu trennen, das, wie oben erwähnt, mit Sicherheit urperm. ${ }^{*}$ voraussetzt. Anscheinend muss man annehmen, dass das $\varepsilon$. das sich im Wotjakischen in der ersten Silbe nur rudimentär erhalten hat, sich irgendwann über sein ursprüngliches Gebiet hinaus ausgebreitet hat. Von diesem $\varepsilon$ ist etymologisch zu trennen das bei Munkácsi in vielen Lehnwörtern aus dem Tatarischen vorkommende $\varepsilon$, das unmittelbar auf der Aussprache in der darleihenden sprache beruht.

a) ${ }^{*} \varepsilon<$ vorperm. $* \ddot{a}: \operatorname{sep~(265),~sektalni~(283),~lésedni~}$ (284; das damit gewöhnlich zusammengestellte wotj. leśtini 'verfertigen' $\sim$ tscherW la•štęm, O lâštę $\cdot m$ id. kann auch ein

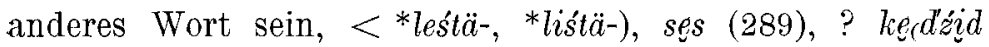
(297). Möglicherweise gehört noch wotj. tei, (Munk.) täj (273) 
hierher; dann hätte im Syrjänischen eine unregelmässige Lautentwicklung $*^{*}>0$ stattgefunden. Wir haben somit $4-6$ Beispiele.

b) ${ }^{*} \varepsilon<$ vorperm. ${ }^{*} e$ : ber (326), kertni (346). In beiden Wörtern kann man wohl eine sporadische Lautentwicklung ${ }^{*} e>{ }^{*} \varepsilon$ vor $r$ annehmen; das Offenwerden von Vokalen in dieser Stellung ist eine äusserst bekannte Erscheinung.

Ausser den obigen gibt es noch einige ${ }^{*} \varepsilon$-Wörter, die Entsprechungen in den wolgafinnischen Sprachen haben, aber wir übergehen sie, weil ihr ursprünglicher Vokalismus sich sehr schwer rekonstruieren lässt.

Anhang zu den Kapiteln über urperm. $\dot{o}$ und ${ }^{*} \varepsilon$.

Infolge der geringen Verbreitung einiger Wörter ist es wenigstens vorläufig unmöglich, zu wissen, ob in ihrer ersten Silbe urperm. $\dot{o}$ oder ${ }^{*} \varepsilon$ gestanden hat. Wir nennen hier folgende Wörter, in denen der Vokal der ersten Silbe auf vorperm. *ä zurückgeht: ? pek (262; in diesem Wort zeigt sich ein merkwürdiges lautliches Schwanken, als ob es von altersher zwei Varianten gegeben hätte: eine mit * $\ddot{a}$, von der fi. päkkä, IpL pàhlkke und syrj. pek herkommen, und eine zweite mit einem engen Vordervokal $*_{i}$ bzw. ${ }^{*} \ddot{u}$, auf die mord. ṕelie und ostj. pŏki zurückgehen), kem (292) und verk (296; an und für sich könnte das syrj. $e$ auch auf ${ }^{*} \dot{o}$ zurückgehen, aber bei diesem ursprünglich vordervokalischen Wort kann man eine solche Möglichkeit nicht ernstlich in Betracht ziehen).

Urperm. $\dot{o}$ und ${ }^{*} \varepsilon$ sind die hauptsächlichen Fortsetzungen von vorperm. ${ }^{*} \ddot{a}$ und haben sich offenbar verhältnismässig spät voneinander getrennt.

$$
\text { 9. Syrj. (auch PO) } e \sim \text { wotj. a) } e, \text { b) } o \text {. }
$$

Wichmans gibt als Zahl der Wörter, die das Lautverhältnis syrj. $e \sim$ wotj. $e$ aufweisen, etwa 40 an (Wotj. Vok. 82), eine Zahl, die zu hoch gegriffen sein dürfte; etwa 30 küme sicherlich der Wirklichkeit näher. Für das Lautverhältnis 
syrj. $e \sim$ wotj. o gibt es nach Wichmann gleichfalls etwa 40 Beispiele (a.a.O.S. 79); diese Schätzung erscheint glaubwürdig.

Welche Veranlassung besteht denn, die Lautverhältnisse syrj. $e \sim$ wotj. $e$ und syrj. $e \sim$ wotj. o zusammen zu behandeln? Erstens ist festzustellen, dass das erste der beiden Lautverhältnisse zwei verschiedene urperm. Vokale $\left(e\right.$ und ${ }^{*} e$ ) vertritt, deren Frequenz unnatürlich gering gewesen wäre, wenn die Gesamtzahl der sie vertretenden Vokale sich auf etwa 30 gemeinpermische Wörter beschränken würde. Zweitens ist der Lautbestand der wotjakischen Wörter, die das Lautverhältnis syrj. $e \sim$ wotj. o vertreten, derart, dass man in ihnen leicht eine Velarisierung und Labialisierung des ursprünglichen $e$ zu o annehmen kann. Drittens erweisen sich die Wörter, die das ebengenannte Lautverhältnis repräsentieren, in den meisten Fällen deutlich als ursprünglich vordervokalisch, soweit sich Entsprechungen für sie in anderen fi.-ugr. Sprachen finden. Dieser Umstand bestärkt uns in der Auffassung, dass das wotj. o durch eine späte Lautentwicklung aus dem im Syrjänischen erhalten gebliebenen Vordervokal entstanden ist. Viertens, und das ist entscheidend, erscheinen als Entsprechungen des wotj. o im Syrjänischen sowohl $e$ als auch $* e$. Das kann nur so ausgelegt werden, dass die beiden $e$-Vokale im Wotjakischen zusammengefallen sind, worauf frühurwotj. $e$ in einem Teil der Vorkommensfälle zul $o$ geworden ist.

Der Unterschied zwischen den beiden $e$ ist in der heutigen Sprache lediglich im Ostpermjakischen sichtbar, wo ursprüngliches $e$ sich erhalten hat, ursprüngliches * $e$ zu $i$ greworden ist. Vgl. Vir. 1951 S. 445 . Sehr interessant ist, dass es in den alten syrjänischen Sprachdenkmälern Texte gibt, in denen $e$ und $\ell$ verschieden geschrieben sind, s. Lүткм Древненермский язык 90-91. Lytkin bezeichnet in der von ihm transliterierten Wortauswahll aus den altsyrjänischen Texten $e$ mit $\boldsymbol{\theta}$ und $e$ mit $\hat{g}$.

Nun betrachten wir die Lautvertretung des urperm. $e$. Obwohl wir längst nicht in allen Fällen die Möglichkeit haben, $e$ und $*_{e}$ zu unterscheiden, weil nur ein Teil der Wörter im 
Ostpermjakischen bekannt ist bzw. in den altsyrjänischen Texten vorkommt, lässt sich schätzen, dass urperm. $e$ in etwa 15 Wörtern erscheint, die das Lautverhältnis syrj. $e \sim$ wotj. $e$ aufweisen, und in etwa 25 Wörtern, die das Lautverhältnis syrj. $e \sim$ wotj. o aufweisen, so dass wir also ungefähr 40 gemeinpermische $e$-Wörter haben.

Auf Grund ihres Auftretens im Altsyrjänischen bzw. Ostpermjakischen können wir als $e$-Wörter wenigstens folgende feststellen: syrj. bed, bed 'Stock, Stab', PO bed', wotj. bodị; syrj. berde 'zu, an', PO bérde, wotj. bordi; syrj. bergalni 'sich wenden, sich drehen', PO b'érgal-, wotj. bergan $\hat{\imath}$; syrj. d'zeskid 'eng', PO žésket; syrj. e-: esten 'dort, hier', eta 'dieser', usw.. PO e-: ésten, sta usw.; syrj. eskini 'glauben', PO (LYTK.) jéskine, wotj. oskịn $\hat{\imath}$; syrj. eštini 'fertig werden', PO jessti-; syrjP gerja'ıni 'laut weinen oder klagen', PO gérjal-; syrj. jen 'Gott'. PO jen, wotj. in; syrj. kel 'bleich, blass', PO kéldi-, wotj. kelit; syrj. ker 'Balken', PO ker, wotj. kor; syrj. keralni 'hauen'. PO kéral-, wotj. koranî; syrj. kerẹs 'Berg, Hügel, Anhöhe'. PO kéres; sỵj. keslini 'schleifen, wetzen, schärfen', PO késli-: syrj. lebni 'fliegen', PO lébal-, wotj. lobni ; syrj. let's 'Schlinge. Dohne', PO leć; syrj. lestśid 'scharf', PO léćot, wotj. le t'sị̂t: syrjI lem 'Leim', PO l'emot-, wotj. lem; syrj. me 'ich', PO me, me, wotj. mon; syrj. med Adverb zur Bezeichnung des Superlativs, altsyrj. мəд, PO med, med, wotj. med; syrj. mezdini 'befreien, erlösen, losmachen', altsyrj. мәз-, $\mathrm{PO}$ mézdi-, wotj. mozmị̂ṇ̂; sỵrj. nerę, nered 'Drüse', $\mathrm{PO}$ nérot. wotj. nored; syrj. herni 'necken, reizen', $\mathrm{PO}$ nér-; syrj. nesjinn! 'niesen', PO riéssot-; syrj. pelis 'Ruder', PO ṕéles; syrj. pe" 'Ohr', PO pel', wotj. pel; syrj. perna 'Halskreuz, Kreuz', PO pérna; syrj. perid 'schmell', PO ṕérta; syrj. pesni 'schlagen', PO ṕés-, wotj. posịn î; syrj. peš 'Kienspanhalter', pešni 'einen Kienspan in den Halter stecken', PO ṕéš-; syrj. petni 'hinausgehen, ausgehen', altsyrj. nəm-, PO ṕét-, wotj. potîn pectśik 'Knips, Schnippchen', PO péćik; sỵj. petśkini 'spinnen', PO péćkelt-; syrjP serp 'Unreinheit, unrein', PO serp: syrj. śera 'bunt', PO séra; syrj. śeral, serol 'Nisse', PO śérel, wotj. śerer; syrj. śeralni 'lachen', PO śéral-, wotj. śerektịn? ; syrj. sermed 'Zaum', PO sérmet, wotj. sermet; syrj. setni 
'geben', altsyrj. c'əm-, PO śét-, wotj. sotîn $\hat{\imath}$; syrj. šebirtni 'decken', PO šébral-, wotj. šobị̂tînnî; syrj. šeg 'Knöchel', PO šeg, wotj. šeg, šeg; syrj. šep 'Ähre', PO šep, wotj. šep; syrj. šerdni 'Getreide durchsieben, Getreide schwingen, worfeln', PO šérdin, wotj. šorźiśkini; syrj. te 'du', PO te, wotj. ton; syrjPO tel 'lichtes, junges Gehölz von Nadelbäumen', wotj. tel; syrj. termedni 'antreiben, beschleunigen', altsyrj. məpмӧd-, PO térmot-; syrj. teś 'gedörrtes, gestossenes (Hafer)mehl', PO teś; syrj. tet'sni 'zusammenlegen', PO téć-; syrjP tetśkini 'ausschlagen', PO téćki-; syrj. t'sepeltni 'kneifen', PO

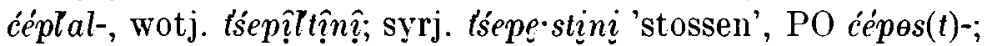
syrj. t'seran' 'Spinne', PO céran', wotj. t'senari, t'sonari; syrj. t'seri 'Fisch', PO cérí, wotj. t'sor $̂$ g; syrj. t'śett'sini 'aufspringen, aufstehen', PO céćći-, wotj. t'śett'sini ; syrj. vekni 'schmal, eng', PO véknit, vékni, wotj. vektši i syrj. verdni 'ernähren', PO vérd-, wotj. vordịnị; syrj. vermịn 'können', altsy̆j. вәрмы-, PO vérmi-, wotj. cormị̂ṇ̂; sỵrj. verźem(-vela) 'Reiter', PO vérzim, wotj. vord'źem(-val); syrj. veś 'vergebens', PO veś; syrj. veśkid 'gerade', PO véśkot, wotj. veśkani; șrj. vešjinn 'wegrücken (intr.), seinen Platz verändern', PO véśji-; syrj. veštini 'loskaufen, auslösen', PO véšti-, wotj. voštịn q̣ (aber: syrj. vežni 'tauschen', PO viz-<*vęz-); syrj. zel 'sehr', P( zélel, wotj. zol; syrj. zer 'Regen', PO zer, wotj. zor; syrj. zerlįd 'rüttelnd, schüttelnd, stossend', PO zérket; sỵrj. žel' $\mathrm{Holz-}$ splitter', PO ž zel. Vol. auch LrTкs Tpy;ы I, SS. 83-84.

Die Frage, wodurch der im Wotjakischen häufige Lautwandel $e>0$ hervorgerufen worden ist, behandeln wir im Lichte umfangreicheren Materials unten auf SS. 312-313.

Etymologien, die die Vorgeschichte des urperm. $e$ erhellen, gibt es nur spärlich.

a) $e<$ vorperm. ${ }^{*} i$ : jen (367), me (373), peš (377), te (388), keralni (398). Möglicherweise gehört hierher noch verdni (401). Wir haben somit insgesamt $5-6$ Beispiele.

b) $e<$ vorperm. *e: eštini (303), pesni (323), pel (344).

c) $e<$ vorperm. ${ }^{*} e$ oder $* \ddot{a}$ : śermed (302).

d) $e<$ vorperm. * $\ddot{a}$ : ? letśs (253).

e) $e<$ vorperm. ${ }^{*} \ddot{u}$ : lem (429). 


\section{Syrj. e, PO $i \sim$ wotj. a) $e$, b) o.}

Von diesem Lautverhältnis war bereits im vorigen Kapitel vorbereitend die Rede. Das urperm. geschlossene $e\left(={ }^{*} e\right)$, um das es sich hier handelt, steht an letzter Stelle in der Frequenzstatistik der perm. Vokale. Von den heutigen syrj. Mundarten hat wenigstens der Ižma-Dialekt Spuren dieses Lautes bewahrt; W ICHMass hat dort nämlich oft $e$ geschrieben, aber dieser Vokal tritt nach seinen Aufzeichnungen auch als Fortsetzung von urperm. $e$ auf; somit scheint der phonologische Unterschied zwischen den beiden $e$-Vokalen verschwunden zu sein. Im Ostpermjakischen hat sich das ${ }^{*} e$ rom urperm. $e$ streng getrennt erhalten; es ist nämlich zu $i$ geworden, und dieses $i$ zeigt seinen vom urperm. $i$ geschiedenen Ursprung darin, dass es in mehrsilbigen Wörtern betont ist, vgl. oben 293. Ganz kürzlich haben wir durch LYTkixs Forschungen wertvolle zusätzliche Angaben über das frühere Vorkommen des $*_{\ell}$ im Syrjänischen erhalten. Das rom $e$ getrennte $\ell$, das er in den alten Sprachdenkmälern festgestellt hat, gibt er mit $\hat{\jmath}$ wieder, vgl. Древнепермский язык 90-91; Труды I, SS. 82-86. Im Wotjakischen ist das * $e$ völlig mit dem $e$ verschmolzen und hat sich wie dieses häufig zu $o$ weiterentwickelt.

Man kann schätzen, dass etwa die Hälfte der Wörter, die das Lautverhältnis syrj. $e \sim$ wotj. $e$ aufweisen, also 15 Wörter, und ebenfalls 15 Wörter, die das Lautverhältnis syrj. $e \sim$ wotj. o aufweisen, urperm. ${ }^{*} e$ gehabt haben, so dass die Zahl der gemeinpermischen $*_{e}$-Wörter etwa 30 beträgt.

Wenigstens bei folgenden Wörtern muss man in der ersten Silbe urperm. * $e$ voraussetzen: syrj. ḍ̌enid 'kurz', PO žinot; syrj. em Suppletivum für 'sein', PO im 'Fülle, Genüge';

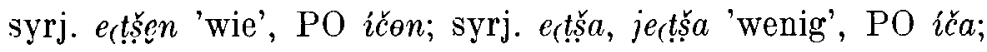

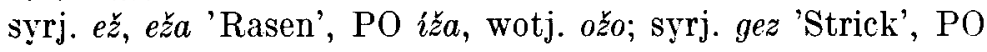
giz, wotj. gozî;; syrj. jem 'Nadel', PO im; syrj. kež, keže 'auf,

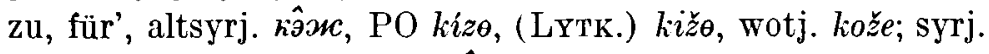

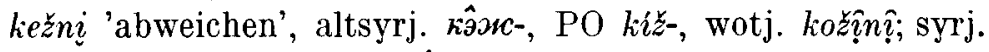

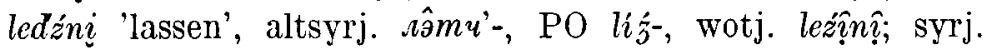
letni 'zupfen, zerren, reissen', PO lit-; syrj. med 'Lohn', PO 
midal-, wotj. med; syrj. nebid 'weich', PO nibot, wotj. nebịt; svrj. pemid 'dunkel, finster', PO pimet, wotj. peimịt; syrj. pes 'Holzscheit', PO pis, wotj. pis (e sporadisch $>i$ ); syrjP pešvinn 'betrachten, probieren, prüfen', PO pišli-; syrj. peźdini 'losgehen (Flinte, Fanggerät)', PO (LYTk.) piźin 'силок', wotj. peźd-; syrj. regidd 'schnell, bald', altsyrj. pâzbə, wotj. dॄ̆og;

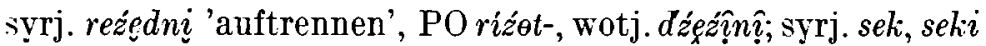
'dann, damals', seten 'dort, da', PO (LYTK.) síki, siten, vgl. wotj. soki, sòtîn $n$; syrj. šedni 'zufallen, zuteil werden, erlangt, erhalten werden', PO (LYTk.) šidlal-, wotj. šedîn $\hat{\imath}$; syrj. tśegni 'brechen', PO ćig-, wotj. tsigîn $̂$ ( $e$ sporadisch $>i)$; syrj. t'ser 'Axt', PO ćir; syrj. vesalni, vese.tni 'reinigen', $\mathrm{PO}$ viset-; syrj. vešja.n 'Hosen', PO víšjan; syrj. vež 'grün, gelb', PO viz, wotj. vož (im syrjänischen gibt es auch das Wort viž 'gelb, grün', aber PO riz gehört nicht damit zusammen, wenn es, wie man glauben larf, etymologisch gleichen Ursprungs ist wie das folgende Wort:) syrj. vez (in einigen Redensarten) 'Neid, Begierde', reželtini 'eifersüchtig sein', PO vižzliti-, wotj. vož 'Zorn, Grimm'; syrj. vež: tui- $v$. 'Wegkreuzung', PO $\imath i z: t y j-v$. , wotj. rož; syrj. vežęr 'Verstand', PO vižor; syrj. vežni 'wechseln, tauschen', PO viz- (aber: syrj. veštini 'loskaufen, auslösen', PO vésti-; dieses Wort wird gewöhnlich für eine Ableitung von režni gehalten). Vgl. auch Lyтkıм Tруды I, S. 91.

Das urperm. ${ }^{*} e$ erweist sich hinsichtlich seines Ursprungs als nah verwandt mit dem im vorigen Kapitel besprochenen $e$ :

a) $*_{e}<$ vorperm. * $i$ : pemid (380), vez 'Neid, Begierde' (391), vež 'grün, gelb' (392), ? vež: tui-v. 'Wegkreuzung' (393). Das sind also $2-4$ Beispiele.

b) ${ }^{*} e<$ vorperm. ${ }^{*} e$ : ? t'segni (351).

c) ${ }^{*} e<$ vorperm. ${ }^{*} \ddot{a} j-\left({ }^{*} \ddot{a} i\right)$ : jem (280). Möglicherweise hat hier die Lautentwicklung $* \ddot{a} j->{ }^{*} i$ stattgefunden und dieses $*_{i}$ hat sich dann ebenso weiterentwickelt wie das ursprüngliche $i$.

Anhang zu den Kapiteln über urperm. ${ }^{*} \varepsilon, e$ und ${ }^{*} e$.

Es seien noch die uns bekannten syrj.-wotj. Wörter genannt, in denen entweder urperm. $e$ oder $*^{*}$ gestanden hat, 
die Qualität des Vokals aber aus dem Grunde, weil Entsprechungen im Ostpermjakischen und in den altsyrj. Texten nicht bekannt sind, sich nicht genauer bestimmen lässt: syrj. lek 'Spielknöchel' $\sim$ wotj. leky; syrj. meg 'Flusskrümmung' $\sim$ wotj. mog; syrj. perjini 'herausziehen' $\sim$ ? wotj. porị̂n? ; syrj. pež 'unrein' $\sim$ wotj. pož; syrj. rektini 'ausräumen' $\sim$ wotj. džoktîn $\hat{\imath}$; syrj. sereg 'die hintere Ecke' $\sim$ wotj. sereg; syrj. seź 'heiteres Wetter' $\sim$ wotj. seź; syrj. śepis 'Sack' wotj. śepị̂; syrj. vetes 'Netz zum Fangen von Enten' wotj. votäs.

Nun können wir die Frage nach dem Lautwandel $e>o$ anschneiden, der im Wotjakischen oft vor sich gegangen ist. Erstens also ist klar, dass urperm. $e$ und $*_{\ell}$ hier anfangs zu $e$ zusammengefallen sind und dass dieses sich dann zu $o$ weiterentwickelt hat. Die Zwischenstufe ist vermutlich ${ }^{*} \dot{o}$ gewesen und das spätere Stadium der Lautentwicklung hat sich gleichzeitig mit dem schon früher (S. 289) behandelten Lautwandel urperm. $\dot{0}>0$ vollzogen. Wir bemerken Folgendes: 1. Von den etwa 30 Wörtern, in denen sich $e$ im Wotjakischen erhalten hat, ohne zu o zu werden, sind 12 solche, in denen unmittelbar auf den Vokal der ersten Silbe ein mouillierter

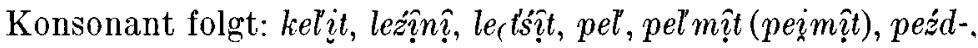

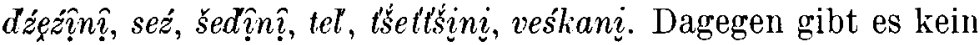
einziges unter den Wörtern, die den Lautwandel $e>0$ aufweisen, in dem auf den Vokal ein mouillierter Konsonant folgen würde. 2. Auch im Anlaut kommt oft ein mouillierter Konsonant in solchen Wörtern vor, in denen sich das $e$ erhalten hat (lem, nebị̂, śepị̂, serer, serelitini, sermet, t'sepị̂t în $n$, tš́ett'šini i insgesamt achtmal), während bei den $a$-Wörtern, ungeachtet der grösseren Gesamtzahl der zu dieser Gruppe gehörenden Wörter, ein mouillierter Konsonant im Anlaut wesentlich seltener ist (nored, sot?̂n ?̂, t'sorị̂g; insgesamt dreimal). 3. In der $o$-Gruppe sind Labialkonsonanten im Anlaut oder unmittelbar nach dem Vokal häufiger (bodî, bord, mon,

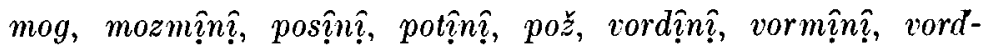
źem, votäs, vož 'grün', vož 'Zorn, Grimm', vož 'Flussmündung, Wegscheide', voštîn in der $e$-Gruppe (bergan ̨ิ, med 'Lohn', med 'dass, damit', 


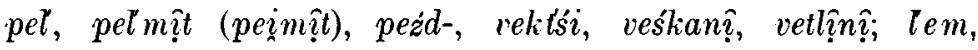
neb?̣̂, śepị̂, šep, t'sepîttîn? ; insgesamt 14mal, aber dabei ist zu beachten, dass achtmal ein mouillierter Konsonant unmittelbar auf den Vokal folgt oder ihm unmittelbar vorausgeht). 4. Dem Vokal vorhergehendes oder auf ihn folgendes

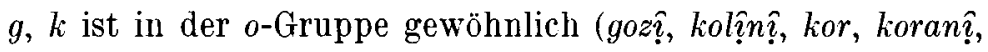

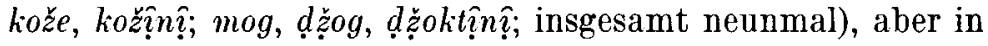
der e-Gruppe recht selten (kelit; leky, veltsi $i$; insgesamt dreimal, wobei in einem Fall auf den Vokal ein mouillierter Konsonant folgt). 5 . Vielleicht kann man zum Schluss auch darauf aufmerksam machen, dass in der o-Gruppe unmittelbar auf den Vokal folgendes $r$ bzw. $z$ recht häufig ist (bord, ožo, kor,

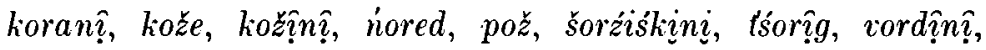
cormîn $\hat{\imath}$, vordżem, vož in seinen verschiedenen Bedeutungen, zor; insgesamt $10 r$ - und $6 \check{z}$-Fälle), aber in der $e$-Gruppe $r$

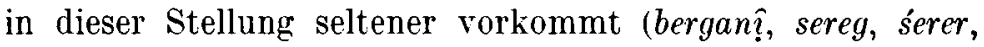
serektini, sermet; insgesamt fünfmal, aber dreimal steht vor dem Vokal ein mouillierter Konsonant) und $\stackrel{z}{z}$ überhaupt nicht.

Auf Grund der oben dargelegten Tatsachen können wir die Behauptung aufstellen, dass im Urwotjakischen eine starke Tendenz zur Labiovelarisierung des $e$ wirksam war. Labialisierend wirkten Labialkonsonanten neben dem Vokal, und auch auf den Vokal folgendes $r$ und $\check{z}$ scheint einen Einfluss in derselben Richtung ausgeübt zu haben; velarisierend wirkten offenbar $g$ und $k$ neben dem Vokal. Intensiven Widerstand leisteten der labiovelarisierenden Tendenz mouillierte Konsonanten in unmittelbarer Nachbarschaft des Vokals, insbesondere wenn sie auf ihn folgten. Der in Rede stehende Lautwandel $e>{ }^{*} \dot{o}$ war trotz seines häufigen Auftretens sporadisch, mit anderen Worten, es gibt Ausnahmen von diesen Regeln, wenn auch nicht zahlreich. So dürften nur zwei Beispiele für einen solchen Lautwandel $e>0$ vorhanden sein, der sich nicht lautlich motivieren lässt: śotîn? zol (ton 'du' ist hingegen offensichtlich durch die Analogie von mon 'ich' bedingt). Etwas mehr Fälle gibt es, in denen sich $e$ erhalten hat, obwohl die umgebenden Konsonanten dem Lautwandel ${ }^{*} e>{ }^{*} \dot{j}$ hätten Vorschub leisten müssen: bergan $\hat{\imath}$, leky (falls 
es nicht *leki ist), med, sereg, šep, vektši, vetlịn $n$. Gab es im gleichen Wort sowohl einen solchen Konsonanten, der die Erhaltung des $e$ begünstigte, als auch einen solchen, der den Wandel desselben förderte, so war gewöhnlich der Einfluss des ersteren stärker als der des letzteren (z.B. kelit, pel; lem. serer usw.; alles in allem 12 Beispiele). Für den umgekehrten Fall haben wir nur zwei Beispiele (nored, t'sorịg).

Als aufschlussreich für die Entstehungsgeschichte der u'perm. Vordervokale seien noch folgende Wörter erwähnt, bei denen man die Qualität des Vokals der ersten Silbe nicht sicher bestimmen kann, weil die betreffenden Wörter in den "Schlüsseldialekten» nicht bekannt sind.

Entweder urperm. $e$ oder $*_{\ell}$ haben folgende Wörter enthalten:

a) < vorpern. $*_{i}$ : $e z$ (365; hier ist wahrscheinlich ein $*_{\ell}$ gewesen, vgl. *vęz< $\left.{ }^{*} v i s ̌ a\right)$, peliś (376; unklar ist, warum die

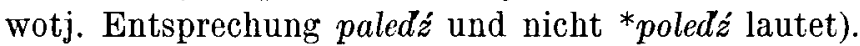

b) $<$ vorperm. ${ }^{*} i$ oder ${ }^{*} e$ : śer $(352$; hier ist wahrscheinlich ein urperm. $e$ gewesen, denn ${ }^{*} e$ ist vor $r$ äusserst selten).

c) < vorperm. ${ }^{*} e$ : nélà $\min (322$; hier liegt wahrscheinlich urperm. *e zugrunde, vgl. pel'< vorperm. ${ }^{*}$ peljä).

d) $<$ vorperm. ${ }^{*} \bar{e}$ : bel' $(359)$.

e) < vorperm. *ä: ? seznis (294), vetes (295).

Urperm. ${ }^{*} \varepsilon, e$ oder ${ }^{*} e$ ist in folgenden Wörtern gewesen:

a) $<$ vorperm. ${ }^{*} i$ : wotj. ped (381; wotj. e kann natürlich auch urperm. ${ }^{*} \varepsilon$ vertreten, aber es erscheint unmöglich, dass es sich hier um etwas anderes handeln könnte als einen der beiden urperm. $e$-Vokale).

b) $<$ vorperm. ${ }^{*} \ddot{a}$ : wotj. ser (301; hier dagegen vertritt das $e$ wahrscheinlich ein urperm. ${ }^{*} \varepsilon$ ).

\section{Syrj. $i \sim$ wotj. $i$.}

Für dieses Lautverhältnis gibt es etwa 60 Beispiele, vgl. WICHMANN Wotj. Vok. 82. Im Ostpermjakischen erscheint hier $i$, das sich von dem urperm. $e$ fortsetzenden $i$ insofern unterscheidet, als es in mehrsilbigen Wörtern unbetont ist, 
s. oben S. 293. Schon im Urpermischen haben die in Rede stehenden Wörter $i$ gehabt.

Die Entstehungsgeschichte des urperm. $i$ ist recht klar.

a) $i<$ vorperm. ${ }^{*} i$ : syrjPO nim- (368), ki: $i z-k i$ (371; im Wotjakischen ausnahmsweise e), nim (374), pin (378), pic (379), ri (384), śiger (385), śin (387), sit (390), wotj. kiźnîn? (394), kiśkyny (395), śiś, siś (399; im Wotjakischen unregelmässig $i>u$ ), wotj. sil' (400), $\operatorname{sim}(402$; wotj. sin- 'rosten' muss davon wahrscheinlich getrennt werden. Anders z.B. UotiLA PK 219). Wahrscheinlich muss man von $i$ auch in folgenden Wörtern ausgehen, in denen die ostseefi. und wolgafi. Sprachen ein sekundäres $\ddot{u}$ haben können: kiśming (411; im Ostseefinnischen ist vielleicht eine sporadische Labialisierung eingetreten: küps- $\left.<{ }^{*} k i p s^{-}\right)$, is (396; im Mordwinischen und Tscheremissischen sehen wir eine anscheinend durch das $p$ verursachte sporadische Labialisierung. Beachtung verdient, dass auch die ugrischen Sprachen auf einen illabialen Vokal hindeuten: Im Ur-Obugrischen ist in diesem Wort ein *e gewesen, das die gewöhnlichste Fortsetzung von fi.-ugr. ${ }^{*} i$ in dieser Sprache war). Wir haben also insgesamt 14-16 Beispiele. - Ein $i(<i)$, das sich nach der urperm. Zeit entwickelt hat, finden wir ausser in $\operatorname{din} \sim \operatorname{din}(430$ : s. oben S. 300) wahrscheinlich auch in wotj. siẓ̂̂ (421), in dem eine Palatalisierung des $i$ vor $z$ gut verständlich wäre.

b) $i<$ vorperm. ${ }^{*} i$ : $\operatorname{iin}(403)$, ? vit (406; hier kann auch ${ }^{*} i$ in Frage kommen. Sollte das $e$ in ret ${ }^{\circ}-\min$ ' 50 ' auf der Analogie von néx à $-\min$ ' 40 ' beruhen?).

c) $i<$ vorperm. ${ }^{*} i$ oder ${ }^{*} e$ : $\operatorname{lin}(307), m i(316)$, $t i(337)$. Bei Pronomina kommt oft sporadischer Vokalwechsel ror: deshalb ist es nicht sicher, ob die obigen perm. Pronomina auf Formen mit $e$ zurückgehen, die den ostseefi. Pronomina genau entsprechen; auch die Lautvertretung im Lappischen und besonders im Mordwinischen erklärt sich vielleicht besser, wenn man ${ }^{*} i$ annimmt. - Unerklärlich bleibt das $i$ in vir (340), falls man hier wirklich von vorperm. ${ }^{*} e$ auszugehen hat. Leichter verständlich wäre eine sporadische Entwicklung *vire >vere- im Ostseefinnischen unter dem Einfluss des $r$. Dabei muss jedoch beachtet werden, dass ein eben- 
solcher Öffnungsprozess, mit anderen Worten, vorperm. $*_{i}>e$, gerade vor $r$ beinahe die Regel in den perm. Sprachen gewesen ist.

d) $i<$ vorperm. * $e$ : sidźni (331), śiźim (332). In diesen Wörtern ist offenbar unter der Einwirkung der benachbarten mouillierten Konsonanten ${ }^{*} e>i$. In sili (334) ist aus dem gleichen Grunde urperm. $i>i$, s. oben S. 302.

e) $i<$ vorperm. *ä: $k i(249$; aber wahrscheinlich handelt es sich um urperm. *e in ke-pis', PO ki-pis' 'Fausthandschuh'), - $v i$ (277). In diesen Wörtern ist der Lautwandel ${ }^{*} \ddot{a}>>i$ wohl das Ergebnis irgendeines Auslautgesetzes, das wir nicht näher kennen. In śiz (240) ist dieser Wandel sporadisch unter dem Einfluss der umgebenden mouillierten Konsonanten eingetreten.

f) $i<$ vorperm. ${ }^{*} o$ : syrjPO nimál (135); hier liegt ein durch das $\dot{n}$ veranlasster Fall von sporadischer Palatalisierung vor.

g) $i<$ Vokal $+j(i)$. Es gibt einige Wörter, in denen ein auf den Vokal folgendes $j(i)$ den Vokal zu $i$ assimiliert hat; gewöhnlich ist der Halbvokal mit diesern $i$ völlig verschmolzen. Z.B. (*-äj->i:) ? bi (261); (*-oj->i:) pi (116), pi-pu (142), man beachte auch $p i, p i i$ (118), in dem ein sekundäres, aus ${ }^{*} \eta$ entstandenes $i$ die Lautentwicklung $*_{0}>*^{*} i$ (diese Stufe ist möglicherwẹise in wotj. Mundarten sogar erhalten, s. oben SS. $302-303$ ) und dann noch $* i>i$ veranlasst hat.

Wie die Beispiele zeigen, ist urperm. $i$ als Fortsetzung von vorperm. $*_{i}$ und $*_{i}$ lautgesetzlich; aus anderen Vokalen ist es lediglich sporadisch entstanden. Steisitz muss unter dem Zwang seiner Theorie das perm. $i$ meistens auf den reduzierten fi.-ugr. Vordervokal (*̌̈) zurückführen, der angeblich mit dem Labialvokal *ŏ verschmolzen sei und zusammen mit diesem zu $i$, aber neben $l$ und $s$ zu $i$ geworden sei, s. Vok. $28-31$.

\section{Abweichende Lautverhältnisse.}

Ausser den oben behandelten 11 Lautverhältnissen, die als normal anzusehen sind, gibt es in den perm. Sprachen eine grosse Menge abweichender Vokalverhältnisse von zufälligem 
Charakter. Wichmass erwähnt (Wotj. Vok. 73-81) 33 Fälle von "sporadischem Vokalwechsel", von denen lediglich sechs normale syrjänisch-wotjakische Lautentsprechungen repräsentieren. In Wirklichkeit ist die Zahl derartiger Fälle von "Vokalwechsel" noch wesentlich grösser als die von Wichmann angegebene, aber da oft für jeden Fall nur ein oder zwei Beispiele vorhanden sind, handelt es sich hier natürlich um ganz zufällige Schwankungen, die lautgeschichtlich weder von Bedeutung noch ron Interesse sind. Einige abweichende Vokalverhältnisse sind jedoch im Wortschatz der permischen Sprachen so zahlreich vertreten, dass Grund besteht, sie gesondert zu betrachten - ausser dem, was schon früher in verschiedenen Zusammenhängen darüber gesagt worden ist. Wir besprechen sie in der Reihenfolge ihrer Häufigkeit, wobei wir uns mit den Beispielen begnügen, die unser eigentliches Forschungsmaterial, d.h. die Wörter, die in anderen fi.-ugr. Sprachen Entsprechungen haben, darbietet.

a) Syrj. $u \sim$ wotj. $i$. Für dies Lautverhältnis gibt es nach Wichmann etwa 35 Beispiele (a.a.O. S. 78), eine Zahl, die allerdings etwas zu hoch gegriffen scheint. Meistens handelt es sich deutlich um einen späten Lautwandel $u>i$ im Wot-

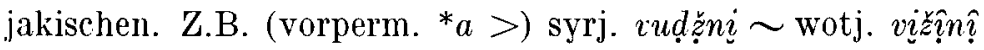

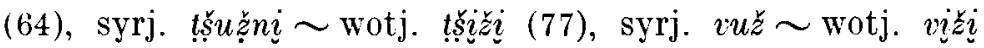
(80), syrj. sulalni, sultni, wotj. sultini $\sim$ wotj. silini (85); (vorperm. ${ }^{*}>$ ) syrj. munda $\sim$ wotj. minda (103).

Es gibt jedoch auch Wortpaare, in denen dieser "Wechsel" offenbar dadurch zustandegekommen ist, dass urperm. $i$ im Syrjänischen, namentlich unter dem Einfluss eines vorhergehenden $k$, zu $u$ velarisiert worden ist: (vorperm. ${ }^{*} u>$ ) syrj. kusni $\sim$ wotj. kị̂sîn $\hat{\imath}(180)$, syrj. kudź wotj. kiddź (181), syrj. kunni $\sim$ wotj. kị̂nị̂n $\hat{\imath}(219)$.

In einigen wenigen Fällen bleibt es unklar, welches von beiden älter ist, das syrj. $u$ oder das wotj. $i$. Solche Wörter sind: syrj. jur wotj. jir (232; wenn dies Wort dem fi. juuri entspricht, dürfte die Lautvertretung im Wotjakischen die ursprüngliche sein), syrj. munni, wotj. munini $\sim$ wotj. $m \underline{i}-$ nini (319; dieser Fall ist deshalb schwer zu beurteilen, weil keiner der beiden perm. Vokale der Lautvertretung im Ost- 
seefinnischen entspricht, obwohl dort eine einzigartige Fülle von Varianten vorliegt $[e \sim \ddot{a} \sim i]$. Es handelt sich wahrscheinlich sowohl im Ostseefinnischen wie in den perm. Sprachen um ursprünglich slangartige Variationen.) Ferner

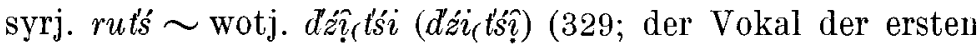
Silbe ist durch Verschmelzung oder Kontraktion entstanden. so dass man ihn nicht unmittelbar mit dem in den westlicheren Sprachen auftretenden $*_{e}$ vergleichen kann).

b) Syrj. $e \sim$ wotj. $i$. Wichmann führt für dieses Lautverhältnis 17 Fälle an, s. a.a.O. S. 75. Diese Wörter sind fast ausnahmslos solche, deren erste Silbe im Urpermischen $*_{i}$ enthalten hat; im Syrjänischen treffen wir also gewöhnlich eine lautgesetzliche Lautvertretung an, aber im Wotjakischen ist spät eine sporadische Lautentwicklung $\left(*_{i}>\right) *_{e}>i$ vor sich gegangen; in einigen Wörtern allerdings hat sich mundartlich auch im Wotjakischen e erhalten. Z.B. (vorperm. ${ }^{*} \bar{o}>$ ) syrj. koz, kez $\sim$ wotj. kị̂z (156), syrj.-wotj. sen $\sim$ wotj $\mathrm{C}$ sịn (163), syrj. ver $\sim$ wotj. vir (166), syrj. seskini $\sim$ wotj. siskini (169); (vorperm. ${ }^{*} a$ oder $*_{o}>$ ) syrj. bež $\sim$ wotj. biž (83). - Einen Wechsel $\dot{o} \sim *_{j} \dot{d}$ weist im Ostpermjakischen das Wort syrj. veini, vetni, PO výj-, vốt'- wotj. vîn? ṇ̂ (50) auf. Keiner von diesen beiden urperm. Labialvokalen passt gut als Fortsetzung des vorperm. ${ }^{*} a$, das in diesem Wort gewesen ist; der Wandel vom Hinter- zum Vordervokal wann er sich auch vollzogen haben mag - beruht auf der sporadischen Einwirkung des darauffolgenden $j$. Vgl. S. 298. - Ein urperm. $\dot{o}$ liegt in syrj. veln $i \sim$ wotj. vilini $(\sim v a l)$ vor (110; vgl. auch S. 291).

c) Syrj. $o \sim$ wotj. $i$ findet man nach Wichmann etwa $15 \mathrm{mal}$, s. a.a.O. S. 77. Sehr interessant ist, dass es sich in wenigstens 15 Fällen um urperm. o handelt, z.B. (vorperm. ${ }^{*} a>$ ) syrjSO kolni $\sim$ wotj. kilini (14); (vorperm. $*_{0}>$ ) syrjSO on $\sim$ wotj $\mathrm{L}$ im (im Wotjakischen im allgemeinen lautgesetzlich $u$ ) (136); (vorperm. ? ${ }^{*} \bar{o}>$ ) syrjSO sol $\sim$ wotj. silal $(161)$; (vorperm. ? ${ }^{*} \ddot{a}>$ ) syrj. von; PO vun $\sim$ wotj. vin $(278)$; (vorperm. ${ }^{*} e>$ ) syrjSO nol $\sim$ wotjM $n_{n} i l$ (aber im wotjG lautgesetzlich $u$ ) (322), syrjSO pon wotjUB p?̂n $\hat{\imath}$ (aber anderswo lautgesetzlich u) (325). Dafür, dass urperm. o im Wotjakischen spora- 
disch zu $i$ geworden ist, gibt es lediglich zwei Beispiele, nämlich (vorperm. ${ }^{*} \ddot{a} \gg$ ) syrj. lokni $\sim$ wotj. liktini $(252)$ und (vorperm. ${ }^{*} e>$ ) syrj. poz $\sim$ wotjMU piz (aber im allgemeinel lautgesetzlich $u$ ) (327).

In den Fällen $\mathrm{a}-\mathrm{c}$ ) handelt es sich also in erster Linie um sporadische Ausbreitung des $i$, das im Urwotjakischen aus dem einen oder anderen Grunde sehr expansionsfähig geworden war, anstelle der benachbarten Vokale $u$ und $\varrho$. Da $i$ in den Fällen, wo es auf einen der beiden urperm. o-Vokale zurückgeht, unstreitig viel häufiger Fortsetzung von urperm. $o$ als von $o$ ist (vgl. auch Foros-Fucus Nyr. LXVI 46), mus: man die Frage aufwerfen, ob im Urwotjakischen $o>u$ früher vor sich gegangen ist als $o>u$ und ob der sporadische Lautwandel $u>\underline{i}$ wesentlich in eine solche Periode fällt, in der bereits zu $u$ geworden, aber o noch erhalten geblieben war.

d) Syrj. $i \sim$ wotj. $u$. Für dieses Lautverhältnis, das Gegenteil des oben unter a) behandelten, gibt es etwa 15 Beispiele. vgl. Wichmann a.a.O. 78. Dazu gehören u.a. (vorperm. ${ }^{*} u>$ ) syrj. kini $\sim$ wotj. luini (183), syrj. pinni, wotj. pinini $\sim$ wotjG punîn̂ (196), syrj. pirzíni 〜 wotj. purd'zini (198): (vorperm. ${ }^{*} \bar{e}>$ ) syrj.-wotj. ńlin $i \sim$ wotjU $n u l \hat{\imath} n \hat{\imath}$ (357), (vorperm. ${ }^{*} \ddot{u}>$ ) syrj. sîl, wotjG sîl $\sim$ wotj U sul, J M sul (422). In all den Fällen, wo sich die Etymologie des Vokals der ersten Silbe klären lässt, hat im Wotjakischen ein sporadischer Lautwandel $i>u$ stattgefunden, dessen junges Alter die Tatsache beweisen dürfte, dass sich mundartlich oft das ursprüngliche $i$ erhalten hat. In unmittelbarer Nachbarschaft des Vokals sind Labialkonsonanten bzw. $k$ sehr gewöhnlich. und auf die labialisierende bzw. velarisierende Wirkung der umgebenden Konsonanten ist der Lautwandel $i>u$ meist wohl auch zurückzuführen.

e) Syrj. $a \sim$ wotj. $u$. Hierfür haben wir etwa 20 Beispiele. vgl. Wichmann a.a.O. S. 74. Die interessantesten Fälle sind unbedingt syrj $m a \sim$ wotj. $m u$ (320) und syrj. $v a \sim$ wotj. $v u$ (342), denen man anscheinend noch syrj. sa 'Russ' $\sim$ wotj. $s u$ an die Seite stellen kann. Es kann wohl kein Zweifel darüber bestehen, dass im Urpermischen in diesen Wörtern (langes?) o bzw. g gestanden hat; einer dieser beiden Labialvokale 
würde ja in der ersten Silbe der Fortsetzungen der Stämme ${ }^{*}$ mete, ${ }^{*}$ vete der Erwartung entsprechen. Im Wotjakischen ist die Entwicklung in der bekannten Weise verlaufen, aber im Syrjänischen scheint irgendein uns unbekanntes Auslautgesetz das Offenerwerden des $o$-Vokals veranlasst zu haben. Es verdient besondere Beachtung, dass nicht immer in Wörtern, die im Syrjänischen zu einsilbigen Vokalstämmen geworden sind, ein derartiger Lautwandel auftritt, z.B. syrj. so, so so 'hundert' $\sim$ wotj. śu.

Entweder von o oder von $o$ ist sicherlich auch in den Wörtern anderen Typs auszugehen, die das Lautverhältnis syrj. $a \sim$ wotj. $u$ aufweisen, aber in ihnen kann das Offenwerden des $o$ in einer ganz anderen Zeit vor sich gegangen sein und auf anderen Ursachen beruhen als in den erwähnten einsilbigen Vokalstämmen. Es wäre natürlich, urperm. $o$ in der ersten Silbe folgender Wörter anzunehmen: (vorperm. ${ }^{*} a>$ ) syrj. vaz $\sim$ wotj. vuz (53); (vorperm. ${ }^{*} a$ bzw. ${ }^{*} o>$ ) syrj. t'śan $\sim$ wotj. t'suni (121).

f) Syrj. $o \sim$ wotj. $a$. Nach Wichmann gibt es für dies Lautverhältnis 18 Beispiele, s. a.a.O. S. 73. In dieser Zahl scheinen jedoch auch 8 Fälle von im Anlaut gewesenem *uo- enthalten zu sein (vgl. Uotila PK 63-64), deren $*_{0}>a$ bereits oben auf S. 280 behandelt wurde. In 7 von den übrigbleibenden Fällen ist urperm. o zugrunde zu legen (s. oben S. 271); zu ihnen gehören u.a. (vorperm. *? o >) syrj. pors 'Schwein' $\sim$ wotj. pars, (vorperm. *? $u>$ ) syrj. moid $\sim$ wotj. mad" (190). In drei Fällen wiederum handelt es sich um ursprüngliches $o$. Von ihnen fällt vor allem (vorperm. ${ }^{*} o>$ ) syrj. sos, SO sos $\sim$ wotj. sajes (137) auf. Dies wotjakische Wort, das 'Ärmel' bedeutet, ist eine Ableitung von sui 'Arm' ( syrj. soi, SO soi $)$. Es ist ganz klar, dass die Sonderentwicklung des $o$ in sajes irgendwie mit der Gesamtstruktur dieses verhältnismässig seltenen Stammtypus zusammenhängt. Die Spärlichkeit des Materials hindert uns daran, ein Urteil darüber auszusprechen, ob $o>a$ in d'źa d'źeg 'Gans'

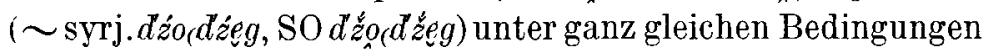
vor sich gegangen ist wie in sajes; jedenfalls herrscht zwischen beiden Wörtern eine grosse Übereinstimmung im Aufbau. 
g) Syrj. $e \sim$ wotj. $u$. Wichmann gibt hierfür 12 Beispiele an (a.a.O. S. 77), aber in Wirklichkeit dürfte ihre Zahl unter 10 liegen. Sechsmal vertritt syrj. $e$ ein ${ }^{*} \dot{a}$, wie in erster Linie die ostpermjak. Entsprechungen dieser Wörter beweisen. Zu diesen Fällen gehören u.a. (vorperm. *? $0>$ ) syrj. pert, PO pyrt $\sim$ wotj. purtî $(131$; wenn dieses Wortpaar dem lp. boarlte entspricht, ist das $u$ im Wotjakischen erwartungsgemäss, aber das * $\dot{a}$ im Syrjänischen bleibt unklar); (vorperm. *? $u>$ ) syrj. neš, PO nyš $\sim$ wotj. nu,tș̌ $\hat{\imath}, n u s ̌ i ~(192 ;$ fi. nuija, nuhja passt hinsichtlich des Vokals der ersten Silbe äusserst schlecht als Entsprechung sowohl des syrj. als auch des wotj. Wortes). Urperm. $\dot{o}$ wird von syrj. $e$ in zwei Wörtern vertreten, deren eines (vorperm. ${ }^{*} \ddot{u}>$ ) syrj. śelem, PO śólom $\sim$ wotj. sulem ist (420; hier steht die Lautvertretung im Syrjänischen offenbar auf einer älteren Stufe als die im Wotjakischen, vgl. S. 291).

h) Für das Lautverhältnis syrj. $o \sim$ wotj. $e$ kennt Wichmann etwa 10 Beispiele (a.a.O. S. 80). Diese kleine Gruppe scheint heterogenen Ursprungs zu sein. Nach dem Zeugnis der ostpermjakischen und der Ober-Sysola-Mundart vertritt syrj. o viermal ein urperm. $o$, so z.B. in den Wörtern (vorperm. ${ }^{*} ? i>$ ) syrj. kośni, SO kos̆alni $\sim$ wotj. kesîn n? (370) und (vorperm. ${ }^{*} \ddot{a}>$ ) syrj. toi , SO toi wotj. tei (273). Der Vordervokal im Wotjakischen erscheint in beiden Fällen ursprünglicher als der Hintervokal im Syrjänischen, falls nämlich die für das Wort kośni beigebrachte Etymologie richtig ist. Diesen Wörtern nahe kommt (vorperm. ${ }^{*}$ ? $i>$ ) syrj. mon, SO moń (Le -meń) wotj. -meń, (WiED. -moń) (372), das insofern merkwürdig ist, als der Wechsel $o \sim e$ sowohl im Syrjänischen als auch im Wotjakischen rorkommt. Wenn wir hier von vorperm. ${ }^{*} i$ (nicht von ${ }^{*} e$ ) auszugehen haben, ist die Form men unstreitig älter als die hintervokalische Form; die Ursache der Velarisierung, die in dieser stattgefunden hat, bleibt unklar.

i) Syrj. $i \sim$ wotj. $i$ und syrj. $i \sim$ wotj. $i$. Diese beiden parallelen Lautverhältnisse, für die Wichmann je etwa 10 Beispiele kennt (a.a.O. S. 81), können wir gleichzeitig betrachten. Sie erweisen sich meist als einfache Palatalisierungsfälle 
$(i>i)$; dieser Prozess ist fast immer durch einen auf den Vokal folgenden Halbvokal $i(j)$ oder einen mouillierten Konsonanten neben dem Vokal hervorgerufen worden. Z.B. syrj.wotj. $p i \sim$ wotj. ( WIED.) pöi<? ${ }^{*}$ pii (118; vgl. SS. 302-303), syrj.-wotj. $\check{s} i \sim$ syrj.-wotj. ši (171), syrj. miślini $\sim$ wotj. miśkîṇ̂̂ (189), syrj.-wotj. vịl $\sim$ syrj.-wotj. vil (215), syrj. siili $\sim$ syrj. sili, wotj. sill (334), syrj. gižz, wotj. giž $i \sim$ wotj. yiži (409), syrj. din, wotj. dịn $\sim$ syrj. din, wotj. din (430).

j) Syrj. $e \sim$ wotj. $e$ kommt nach Wichmann in etwa 10 Fällen vor (a.a.O. S. 80). Diese widerspiegeln zufällige Schwankungen, die mundartlich aufgetreten sind; bald handelt es

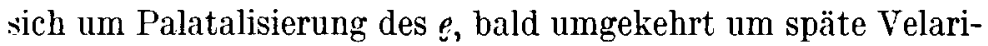
sierung des $e$ wie z.B. in wotj U lem ( strj.-wotj. lem) (429).

Im Vorstehenden haben wir kurz alle sporadischen Fälle ron "Vokalwechsel» betrachtet, von denen mindestens $10 \mathrm{Er}$ scheinungsfälle bekannt sind. Die Vokalvertretung in den permischen Sprachen ist oft sehr bunt, aber diese Buntheit ist ein später Zug, der nichts mit irgendeinem alten »Ablautwechsel) zu tun hat. Wie am Anfang dieses Kapitels festgestellt wurde, repräsentieren von den 33 bei Wichmann angeführten "Vokalwechsel»-Gruppen ganze 27 abweichende Lautverhältnisse, die hauptsächlich auf zufälligen Reihenübergängen beruhen. Diese 27 Gruppen enthalten durchschnittlich je 7 Wörter. Schon aus dieser niedrigen Frequenz ersieht man ohne weiteres, dass sie keine selbständigen urperm. Lautkategorien vertreten können. Vergleichshalber sei erwähnt, dass die durchschnittliche Frequenz der oben behandelten wirklicheIr urperm. Vokalkategorien 70 Wörter beträgt, also das Zehnfache der Frequenz der abweichenden Gruppen.

13. Die Behandlung der einzelnen vorpermischen Vokale im Urpermischen.

$$
* a \text {. }
$$

a) $u$ : 17-21 Beispiele (ul, udni, jukni, kulem, lud, mus, bur, suni, ? suk, turin, dugdini, vug, vudžni, ? mudźni, sudźni, ț̣suz̆ni, vuž, juvalni, sulalni; ? juskini, ? pur; s. S. 286).

b) o: 9-14 Beispiele (olitini, vośkol, ? koini, kolni, goz, 
šor, koźin, ozir, roź; òz ? wotj. kuaț̣̆kànị; ? vojny, ? moị, 'nokt'sim; s. SS. 279, 281, 285).

Von diesen zwei Lautvertretungen ist lediglich $u$ lautgesetzlich, aber das dem $u$ qualitativ nahstehende $*_{o}$ ist sehr oft durch sporadischen Reihenübergang zur Fortsetzung von ${ }^{*} a$ geworden.

Die übrigen Vertretungsweisen sind vereinzelte Fälle von ranz niedriger Frequenz; es gibt für jede nur ein oder zwei Beispiele und auch sie sind zum Teil unsicher:

c) a: bad, ?? vartni, s. S. 265 .

d) o: sorni, s. SS. $272-273$.

e) $i$ : penni, s. S. 291.

f) ${ }^{*} \dot{d}:$ ? bez, s. S. 297 .

g) $\dot{o} \sim *^{*}$ : veini, s. SS. 298,318 .

h) $i$ :? kik (es ist nicht sicher, ob man hier geradezı vol vorperm. ${ }^{*} a$ auszugehen hat, s. S. 303), ? wotj. ibini, ? wotj. tilis (hier kann das $i$ sich nach der urperm. Zeit sporadisch aus $u$ entwickelt haben, s. S. 303).

$$
\text { *o. }
$$

Vorperm. ${ }^{*} o$ ist völlig mit vorperm. ${ }^{*} a$ verschmolzen, so lass also auch es auf folgende Weisen vertreten wird:

a) $u$ : 16-24 Beispiele (? ju, juk, juś, kud, kuț̣̆, lun, munda, ? nu(l)ni, ur, vuz, śur, ? (dod'-)śul, śul(-t'se $\left.e^{k}\right)$, śul 'Darm', nur, lul, ț̣̆už, kuź, puly(-pom); ? juskini, ? pur, ? uz, ? $u(v) t n i$ $\because$ uini, s. SS. 286-287).

b) $o:$ :-10 Beispiele (vòd $\dot{z}$, pòz, on, soi; ko, ko-min, kor, $\because k o s-;$ ? $k o s(k)$, ? tolti, s. SS. 279, 281, 285).

Vereinzelte Fälle:

c) *jo: 3-5 Beispiele (vosni, los, wotj. vęi; ? bezz; ? nèzz, s. SS. 297, 298-299), die teils auf einer sekundären Dehnung des $*_{o}$ in irgendeinem früheren Stadium, teils auf dem palatalisierenden Einfluss der umgebenden Konsonanten beruhen.

d) o: nort, voz, ? porś. Hier handelt es sich wahrscheinlich IIm ein sporadisches Offenwerden des $*_{a}$ ror $r, \check{z}$, s. S. 272.

e) $\dot{0}: v e \ln i$, s. SS. $291,318$.

f) $a$ :?? kad, s. S. 266. 
${ }^{*} \bar{o}$.

a) ${ }^{*} \dot{a}:$ 7-9 Beispiele (kez, nel, sem, sen, l'em, velalni, ver, ? demni, ? seskini, s. SS. $296-297)$. ${ }^{*} \dot{a}$ ist die einzige lautgesetzliche Fortsetzung von ${ }^{*} \bar{o}$.

Vereinzelte Fälle:

b) $u$ : kulni, pul; ? kun, s. SS. 287, 302.

c) $o$ : ? sol; vo-, s. SS. $280,281$.

$$
* u \text {. }
$$

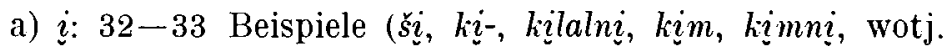
kidź, wotj. kisinin, kini, lid, lịm, li, miskini, bitşkini, pinni,

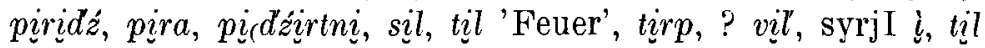

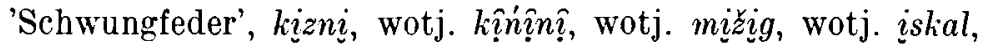
miž, nill, pides, pirni, piž, tiki, s. S. 300).

Die übrigen Vertretungsweisen, die neben diesem selten regelmässigen und in einer grossen Anzahl von Beispielen eingetretenen Lautwandel vorkommen, sind zahlenmässig bedeutungslose Ausnahmen; das spärliche Material ist teilweise etymologisch unsicher:

b) $u$ : purni ( $u$ zwecks Vermeidung von Homonymie), ?vunni, ? uini, s. S. 287.

c) $0:$ ? ? jon, ? moid, s. S. 273.

d) $o:$ ? kot, s. S. 279 .

e) $\dot{0}: t e d n i$, s. S. 291.

$$
{ }^{*} \bar{u}
$$

a) $i$ : 3-4 Beispiele (kilni, sinni, $i z$, ? wotj. jir, s. S. 302.)

b) $u$ : 1-5 Beispiele (juni, ? pu, ? wotj. su, ? jur, ? kun, s. S. 287). Die Lautvertretung mit $u$ scheint in Wörtern in Frage zu kommen, die zu einsilbigen Vokalstämmen geworden sind.

c) ${ }^{*} \dot{a}: t e l$, wo der unregelmässige Vokal pro $i$ zweifellos durch das Bestreben veranlasst ist, Homonymie zu vermeiden, s. SS. $297-298$. 
$* \ddot{a}$.

a) $\dot{o}:$ 6-10 Beispiele (jez, kesjini, ? remis, ? pek, reped. tel, pel, evedz; ? kem, ? verk, s. SS. 291, 306).

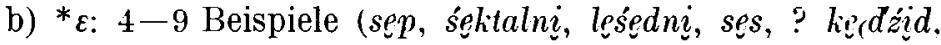
? wotj. tei; ? wotj. ser; ? kem, ? verk, s. SS. 305-306, 321; 314 . 306).

c) $a: 3-7$ Beispiele (na, ta-, ai, ? malalni, ? wotj. naani,

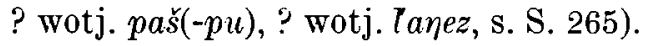

d) o: 3-6 Beispiele (lokni, pon, oktini; ? jos, ? lop, ? lol(-pu), s. SS. $271-272$ ).

e) $i$ : 3 Beispiele ( $k i,-\imath i$ [in diesen Wörtern ist offenbar irgendein besonderes Auslautgesetz wirksam gewesen], śiź [hier ist $* \ddot{a}>>i$ durch den Einfluss der umgebenden mouillierten Konsonanten rerursacht wordenl, s. S. 316).

f) e: 4 unsichere Beispiele (? sermed, ? let's; ? seźnis, ? vetes, s. SS. $309 ; 314$ ).

g) $*_{e}:$ ? seźnis, ? vetes, s. S. 314 .

Die gleichmässige Verteilung des vorperm. * $\ddot{a}$ auf viele im Urpermischen entstandene Vokalkategorien ist eine der interessantesten Erscheinungen der Entwicklungsgeschichte des perm. Vokalsystems, die sich nur vor dem Hintergrund der Systemganzheit erklären lässt, vgl. S. 339.

$* e$.

a) $o: 7-8$ Beispiele (kor, ? kos, lol, moš, nol, polni, pon. soing, s. S. 277 ).

b) o: 6-10 Beispiele (olni, ? korni, poz, požem, t'sož, ? mort, ord-, kolni; ?lop, ? lol(-pu), s. SS. 271, 272).

c) e: 3-6 Beispiele (eštini, pešni, pel; ? nęlà-mîn, ? ser. ? sermed, s. SS. 309, 314).

Diese drei Arten der Lautvertretung kann man als normal betrachten: Die beiden ersten widerspiegeln die Velarisierung des ${ }^{*} e$ zu einem geschlosseneren oder offeneren $o$ je nach der ursprünglichen Qualität des Vokals der zweiten Silbe; die dritte Gruppe enthält die Fälle, in denen das $e$ von der Velarisierung verschont blieb, was gewöhnlich mit einem mouillierten Konsonanten neben dem Vokal zusammenzuhängen 12- Finnisch-ugrische Forschungen 
scheint (wenn das $j$ im Anlaut von eštini in den permjakischen Mundarten ein urperm. Zug ist, lässt sich die Erhaltung des $e$ verstehen, weil es ja auf das $j$ folgte).

Vereinzelte Fälle:

d) $i: 2-5$ Beispiele (sidźni, šiźzm |durch die mouillierten Konsonanten veranlasste sporadische Schliessung], ? kin,? $m i$, ? $t i$, s. SS. $315-316)$.

e) ${ }^{*} \varepsilon$ : ber, kertni (durch das $r$ hervorgerufenes sporadisches Offenwerden des Vokals), s. S. 306.

(Unsichere:)

f) $a$ :? mal, ? wotj. vaź, ? zarnii, s. S. 265.

g) ${ }^{*} e$ : ? t'segni, s. S. 3I1.

\section{${ }^{*} \bar{e}$}

a) $i$ : 5 Beispiele (kil, lim, mil, $\dot{n} \imath \ln i$, nir, s. S. 301).

b) $e$ bzw. ${ }^{*} e$ : bel (die Erhaltung des palatalen Charakters des Vokals beruht offenbar auf sporadischer Einwirkung des $l$ ), s. S. 314 .

$$
* i \text {. }
$$

a) $i$ : 14-19 Beispiele (n'im-, ki, nim, piń, piv, ri, siger, sin, sit, wotj. kizṇ̂ṇ̂, kiśkyny, siś, wotj. sil, sim, ? kiśmini, ? is; ? kin,? mi,? ti, ? vit, s. S. 315).

b) e: 5-6 Beispiele (jen, me, peš, te, keralni, ? verdni, s. s. 309).

c) *e: 2-4 Beispiele (pemid, vežz 'Neid, Begierde', vez 'grün, gelb' [diese Homonyme können verschiedene Bedeutungen ein und desselben ursprünglichen Wortes vertreten], ?vež : tui-v. 'Wegkreuzung', s. S. 311).

d) $e$ bzw. ${ }^{*} \ell: 4-5$ Beispiele ( $e z$ thier wahrscheinlich $\ell$ ], peics, ser [hier wahrscheinlich e], wotj. ped; ? veti-min, s. SS. 314, 315).

Die erste dieser vier Gruppen vertritt die normale Fortsetzung von vorperm. ${ }^{*} i$, die drei übrigen widerspiegeln das Offenwerden des $*_{i}$, das in erster Linie sporadisch gewesen zu sein, aber jedenfalls vor $r$ und $\check{z}$ sehr häufig vorgekommen zu sein scheint (s. darüber näher SS. 338-339). 
Vereinzelte Fälle:

e) $o$ : potș (Übergang in die ${ }^{*} e$-Reihe, s. S. 272).

f) $\underline{i}$ : šir, rịniš (anscheinend ursprünglich sporadische Labialisierung $*_{i}>*^{*} \ddot{u}$, s. S. 303).

a) i: nin, ? vit, s. S. 315 .

b) $a(i)$ : vaini, s. S. 265 .

c) ? e bzw. $e$ : veti $-\min , \mathrm{s} . \mathrm{S} .315$.

So überaus spärlich das Beispielmaterial auch ist, wahrscheinlich ist jedenfalls, dass nur die erste Vertretungsweise normal ist.

$$
\text { *ii. }
$$

a) $i$ : 15 Beispiele (kin, giž, wotj. kitkini, wotj. kiï, wotj.

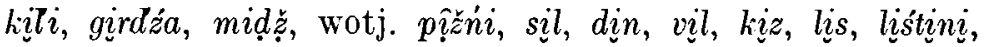
$m i k$, s. SS. 300-301.) Dies ist die einzige lautgesetzliche Fortsetzung von * $\ddot{u}$. Andere Vertretungsweisen sind:

b) $\dot{o}: 4$ Beispiele (śelem, śelalni, et, šert [hauptsächlich sporadisches Offenerwerden des $*_{\ddot{u}}$ vor Liquida, s. S. 291]).

c) $i$ : ? kiśmini, ? is (in diesen Wörtern ist möglicherweise ursprünglich *i gewesen), ? wotj. siž?l (hier wahrscheinlich sekundär urperm. $\left.{ }^{*} i>i\right)$, s. S. 315 .

d) $e$ : Yexm (die Fortsetzung des ${ }^{*} \ddot{u}$ ist offenbar unter dem Einfluss des $l$ palatal geblieben) s. S. 309 .

e) 0 : ? sodni, ? oi (wenigstens in dem letzten Wort ist möglicherweise von vorperm. ${ }^{*} e$ auszugehen und nicht von $*_{\ddot{u}}$, das in den fi.-wolg. Sprachen sekundär sein dürfte), s. SS. 279 -280 .

Das allgemeine Bild von der Weiterentwicklung der vorperm. Vokale im Urpermischen bleibt bunt, wenn man der Frequenz der verschiedenen Vertretungsweisen keine Beachtung schenkt. Betrachtet man dagegen die Dinge im Lichte der Frequenzverhältnisse, so werden die Entwicklungslinien für jeden Vokal sogar überraschend deutlich sichtbar; die sonderentwicklungen, Reihenübergänge und sonstigen zufäl- 
ligen Schwankungen bleiben ihren Frequenzziffern nach kleine Minderheiten. In den verhältnismässig seltenen Fällen, in denen eine gleichmässige Aufteilung des Urvokals auf verschiedene Vokalkategorien vorliegt, ist dieser Aufspaltungsprozess offenbar für die Herausbildung des urperm. Vokalsystems nötig gewesen, wie im 15. Kapitel näher erläutert werden wird.

\section{4. İ̉ber die Frequenz der Vokale.}

Gemeinpermische Wortstämme, die sowohl im Syrjänischen als auch im Wotjakischen vorkommen, kennen wir nahezu 1000 , eine Zahl, die ungefähr der Anzahl der gemeintscheremissischen Wortstämme entspricht. In diesem Material sind die Frequenzen der urperm. Vokalkategorien in der ersten Silbe, prozentual ausgedrückt, folgende: $u 15, i 13, o 8, a, o$ und ${ }^{*} j 7, i 6, \dot{0},{ }^{*} \varepsilon$ und $e+,{ }^{*} e 3^{0}{ }_{0} ;$; unklare Lautverhältnisse $21^{\circ} . \mathrm{Zu}$ der letztgenannten Gruppe sei bemerkt, dass sie den grössten Teil jener abweichenden Vokalverhältnisse enthält, deren gewöhnlichste Typen oben im 12. Kapitel betrachtet worden sind. Einige grössere Gruppen unter den abweichenden Lautverhältnissen lassen sich jedoch lautgeschichtlich klären und zwar mit dem Ergebnis, dass die Stellung der Vokale, die an der Spitze unserer Aufzählung stehen, gestärkt wird. Stellen wir neben diese Zahlen die Frequenzverhältnisse der urperm. Vokale in jenem ältesten Wortmaterial, das etymologische Entsprechungen in den fi.-wolg. Sprachen hat. Dies Material umfasst insgesamt etwa 300 Wörter und bei etwa 250 von ihnen kann man feststellen, welcher Vokal im Urpermischen in der ersten Silbe gestanden hat. Die Statistik ist folgende: $i 27, u 19, o 15, i 11, o 6, \dot{o}$ und $* \dot{o} 5, e 4, a$ und $* \varepsilon 3, \& 2 \%$.

Da das nächste Ziel unserer L'ntersuchung die Klärung der Entstehungsgeschichte des urperm. Vokalismus ist, interessieren uns in diesem Zusammenhang am meisten die Frequenzverhältnisse der Vokale in dem aus vorperm. Zeit stammenden Wortmaterial. Man muss nachdrücklich darauf 
hinweisen, dass das von uns behandelte Material, das aus älterer als der urperm. Zeit stammt, unvollständig ist: In ihm fehlen ja solche Wörter, die Entsprechungen in den ugr. (oder in den samojed.) Sprachen haben, aber nicht in denjenigen fi.-ugr. Sprachen, die westlich von der permischen Gruppe gesprochen werden. Anderseits besteht jedoch Veranlassung, sich daran zu erinnern, dass, auch wenn man eine Statistik aufstellen wollte, die auf einem vollständigen Wortmaterial fusst, der knappe permisch-ugrische Sonderwortschatz sicherlich keine tiefgreifenden Änderungen in die oben angegebenen Frequenzzahlen bringen könnte. Er könnte das schon aus dem Grunde nicht, weil wenigstens im heutigen Stadium der Forschung die genaue Bestimmung der vorperm. Vokalqualitäten sehr schwer fallen kann, wenn es nur aus ugrischen Sprachen Vergleichsobjekte gibt; somit würde ein erheblicher Teil eines solchen vollständigen Materials für die Forschung nutzlos bleiben und die Gruppe der "unklaren Lautverhältnisse» vermehren.

Noch eine dritte Statistik sei vorgelegt, nämlich über die Frequenz der vorperm. Vokale in dem 210 Wörter der perm. Sprachen umfassenden Material, bei dem sich die vorperm. Vokalqualität mit Sicherheit bestimmen lässt: ${ }^{*} a$ und $* u 15$, $*_{o} 14, *_{\ddot{a}}$ und $*_{i} 12, *_{e} 10, *_{\ddot{u}} 9, *_{\bar{o}} 5, *_{\bar{u}}$ und $*_{\bar{e}} 3, *_{i} 2 \%$. Wenn man sich daran erinnert, aus welchen Elementen sich jeder urperm. Vokal gebildet hat, kann man feststellen, dass die Frequenzzahlen der vorperm. und der urperm. Vokale einander gut entsprechen. Berechnet man z.B. die Gesamtfrequenz von vorperm. ${ }^{*} u,{ }^{*} \ddot{u},{ }^{*} \bar{u}$ und ${ }^{*} \bar{e}$, so erhält man ein Ergebnis von $30 \%$; in der urperm. Sprache kam die Prozentzahl des $i$, das zur hauptsächlichen Fortsetzung der genannten Vokale geworden war, diesem Wert sehr nahe, sie betrug nämlich 27. Ebenso entspricht den Erwartungen die Prozentzahl 19 des urperm. $u$, wenn wir wissen, dass vorperm. ${ }^{*} a$ und ${ }^{*} o$, deren Gesamtfrequenz sich auf $29 \%$ beläuft, ungefähr in zwei Dritteln der Vorkommensfälle zu $u$ geworden sind. Usw.

Die Frequenzziffern der vorperm. Vokale bilden eine gleichmässig absteigende Reihe: Ohne irgendwelche grösseren 
Sprünge stehen am Anfang die drei kurzen Hintervokale und danach die vier kurzen Vordervokale; eine deutliche Schwelle erscheint erst $z$ wischen den kurzen und langen Vokalen. Die Frequenz der langen Vokale ist erwartungsgemäss viel kleiner als die der kurzen; in beiden Gruppen sind die Hintervokale etwas stärker vertreten als die Vordervokale. Alles in allem hat im vorperm. Vokalismus nach unserem Material ein knappes Übergewicht der Hintervokale geherrscht, die $52 \%$ gegenüber $48 \%$ der Vordervokale ausmachten. Man kann annehmen, dass erst allmählich eine derartige Harmonie und Folgerichtigkeit erreicht worden ist, als Ergebnis instinktiver Tendenzen, die Jahrhunderte, vielleicht Jahrtausende hindurch in der Wortschöpfung wirksam gewesen sind.

Ein ganz anderes Gesamtbild öffnet sich vor uns, wenn wir die Frequenz der urperm. Vokale im ältesten Wortgut der Sprache betrachten. Hier gibt es keine Spur von Harmonie. Das frühere vorperm. System ist anscheinend in recht kurzer Zeit gründlich zerfallen und das aus seinen Trümmern zusammengestellte neue System ist so unausgeglichen wie nur möglich. An der Spitze erhebt sich wie ein einsamer Berggipfel

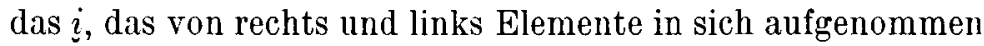
hat; darauf folgen, gleichsam durch hohe Treppenstufen voneinander getrennt, $u, o$ und $i$; in weitem Abstand kommen dann, schwach vertreten, die übrigen Vokale, sieben an der Zahl. In unserem Material beträgt die Gesamtfrequenz der letztgenannten Laute, die die Mehrheit des Vokalsystems bilden, $28^{\circ}$, was ungefähr der Frequenz des $\underset{i}{i}$ allein $(27 \%$ gleichkommt. Die Vokalqualität hat einen interessanten Wandel durchgemacht: Die frühere Zweiteilung in Hinter- und Vordervokale ist verschwunden, indem sich zwischen beiden Gruppen die neue Kategorie der Mittelvokale herausgebildet hat. Ihre Entstehung bedeutete vor allem für die Vordervokale einen grossen Aderlass, wie die Gesamtprozentzahlen der verschiedenen Gruppen zeigen: Hintervokale 40, Mittelvokale 37 und Vordervokale 23. Es dürfte lehrreich sein, hier an die Frequenz der urtscher. Vokale in dem aus vortscher. Zeit stammenden Wortmaterial zu erinnern. So fühlbare Veränderungen die einzelnen Vokale auch in diesem System 
durchgemacht haben, ist die Entwicklung doch nicht so schroff in neue Bahnen gerissen worden wie im Permischen. Daher haben sich die vom vortscher. System ererbte gleichmässig absteigende Reihe der Frequenzziffern der Vokale und die knappe Mehrheit der Hintervokale gut erhalten.

Der gemeinpermische Wortschatz der heutigen Sprachen, dessen ganz überwiegender Teil zweifellos das Ergebnis einer erst in urperm. Zeit vor sich gegangenen Wortschöpfung ist, scheint die Tendenz aufzuweisen, die anfangs in der Frequenz der urperm. Vokale aufgetretenen grossen Ungleichmässigkeiten zu eliminieren. Das $i$ hat seine führende Stellung an das $u$ verloren, das als einziger der an der Spitze stehenden Vokale seine frühere Frequenz ungefähr bewahrt hat. In der Frequenz des $i$ ist ein sehr starker Rückgang eingetreten, aber auch $o$ und $i$ haben merklich verloren. Hingegen haben die früher am Ende der Reihe stehenden Vokale, die bis dahin sehr schwach vertreten waren, ihre Stellung verbessert, der eine mehr, der andere weniger. Am grössten ist der Zuwachs bei a gewesen, das, wie wir schon eingangs (S. 266) feststellten, hauptsächlich im späten perm. Wortgut vorkommt. Das Ergebnis ist eine recht harmonische Reihe, in der eine Art von plötzlichem Abfall nur zwischen dem zweiten und dritten Vokal der Statistik, d.h. zwischen $i$ und $\underset{\alpha}{o}$, auftritt. Man könnte die Anordnung der verschiedenen Gruppen als Ausdruck des Strebens nach deutlichen Grenzen anslegen: Die Stellung der Hintervokale hat sich verstärkt, die der Mittelvokale ist geschwächt worden und die der Vordervokale ist gleich geblieben. Die wirklichen Zahlen dürften ungefähr folgende sein: Hintervokale 47, Mittelvokale 31 und Vordervokale $22 \%$. Von den einzelnen Vokalen durchbrechen diese Anordnung nur $i$, das zwischen die Hintervokale gedrungen ist, und $i$, das den am schwächsten vertretenen Mittelvokal knapp überholt hat. Wir müssen uns daran erinnern, dass in unserer Statistik der heutigen Sprache die Prozentzahlen der Fortsetzungen der urperm. Vokalkategorien durchweg niedriger als die wirklichen Prozentzahlen sind, weil aus den oben angegebenen Gründen ganze $21 \%$ des Materials ausserhalb der erwähnten Zahlen gelassen wer- 
den mussten. Zweitens besteht Veranlassung, zu unterstreichen, dass die in der heutigen Sprache herrschenden Vokalsysteme - ob es sich nun um das Syrjänische, die permjak. Dialekte oder das Wotjakische handelt - in manchen Einzelheiten keineswegs mehr der Lautvertretung im Urpermischen entsprechen. Von entscheidender Bedeutung ist der Umstand gewesen, dass die Gesamtzahl der Vokale sich überall verringert hat, nachdem mehrere urperm. Vokale miteinander verschmolzen waren. Die Untersuchung der Frequenzverhältnisse der Vokale in den heutigen Sprachen, so interessant sie an sich auch wäre, lassen wir hier beiseite.

15. Der urpermische Vokalismus als phonologisches System und seine Entstehung aus dem vorpermischen System.

\begin{tabular}{|c|c|}
\hline \multicolumn{2}{|c|}{ Vorpermisch } \\
\hline$a$ & $\ddot{a}$ \\
\hline$o-\bar{o}$ & $e-\bar{e}$ \\
\hline$u-\bar{u}$ & $\ddot{u} i-\bar{\imath}$ \\
\hline
\end{tabular}

Urpermisch

$\begin{array}{lll}a & & \varepsilon \\ o & \dot{o} & e \\ o & \dot{o} & \ell \\ u & \stackrel{i}{i} & i\end{array}$

Oben sehen wir zwei Systeme, zwischen denen kaum eine andere grundsätzliche Übereinstimmung besteht, als dass beide 11 Vokale haben. Im urperm. Vokalismus haben sich drei Veränderungen vollzogen, die den Charakter des ganzen Systems bis in die Grundfesten erschüttert haben: 1. Der Quantitätsunterschied zwischen kurzen und langen Vokalen ist verschwunden und die kurze Quantität ist alleinherrschend geworden. (Die mundartlich in einigen Wörtern aufgezeichnete halblange Quantität, z.B. SO pòz 'Nest', hat keine phonologische Bedeutung; ein vom Ganzen aus gesehen bedeutungsloser Sonderfall sind auch die langen Vokale des Ižma-Dialektes, die durch Assimilation von $l$ an den vorhergehenden Vokal entstanden sind, z.B. $p \bar{u}$ 'Preiselbeere' < pul. Auf welche Weise und in welcher Periode die Kürzung der vorperm. langen Vokale stattgefunden hat, davon sich ein genaues Bild zu machen, dürfte unmöglich sein. Jedenfalls 
geht beispielsweise aus solchen Lautverhältnissen wie ostseefi. $o \sim$ urperm. $u(o)$, ostseefi. $\bar{o} \sim$ urperm. ${ }^{*} \dot{o}$ und ostseefi. $e \sim$ urperm. $o, o$, ostseefi. $\bar{e} \sim$ urperm. $i$ deutlich hervor, dass der urperm. Vokalismus auf ein System zurückgeht, in dem eine dem Ostseefinnischen entsprechende und dem gleichen Ursprung entstammende quantitative Reihenaufteilung geherrscht hat, und dass diese Aufteilung noch während der perm. Sonderentwicklung in Geltung gewesen ist.) 2. Die frühere Zweiteilung in Hinter- und Vordervokale ist einer Dreiteilung in Hinter-, Mittel- und Vordervokale gewichen. 3. Ausser den drei Artikulationsgraden, die das vorperm. System bei den Vokalen kannte, weit, halbeng und eng, hat sich im urperm. System noch ein vierter Artikulationsgrad entwickelt, der in der Mitte zwischen dem halbengen und dem engen liegt.

Unsere Rekonstruktion des urperm. Vokalismus kann man wohl für alle Laute ausser $\varepsilon$ als sicher ansehen. Viele Gesichtspunkte legen es jedoch nahe, auch das Vorhandensein des $\varepsilon$ anzunehmen, von denen als ein recht beachtenswerter die Gesamtstruktur des Vokalsystems erwähnt sei. Selten sieht man ein so streng folgerichtiges System, das dabei doch alles andere als einfach ist. Die nichtweiten Vokale bilden drei einander genau entsprechende Reihen, von denen jede einen Hinter-, einen Mittel- und einen Vordervokal umfasst. Was die Reihe der weiten Vokale betrifft, so lässt sich schwer denken, dass zu ihr kein anderer Vokal gehört hätte als $a$ und dass diese Reihe also ganz im Unterschied zu den anderen sehr unsymmetrisch gewesen wäre. Somit entspricht vom Standpunkt der Systemganzheit $\varepsilon$ als Entsprechung von a der Erwartung. Ein drittes, mittelvokalisches Glied hat die weite Vokalreihe ganz offensichtlich nicht gehabt.

Oben im 13. und 14. Kapitel sind ausführlich die vorperm. Vorgänger und die Frequenz der urperm. Vokale angegeben worden. Wir sahen, wie einige Vokale sogar mit vielen starken Wurzeln im vorperm. Boden verankert sind, während der Ursprung gewisser Vokale, die im Wortmaterial schwach vertreten sind, beinahe zufällig wirkt. Die grosse Ungleichmässigkeit der Frequenzzahlen der urperm. Vokale im älte- 
sten Wortgut dieser Sprachform bildet einen krassen Gegensatz zu der qualitativen Symmetrie des urperm. Vokalsystems. Dieser Gegensatz bedeutet in Lichte des Kausalverhältnisses zwischen den beiden Erscheinungen keinen Widerspruch. Im Gegenteil, wir erhalten durch ihn offenbar den passenden Hintergrund für die Entwicklungsgeschichte des ganzen urperm. Vokalismus.

Als im Frühurpermischen das Streben nach grösstmöglicher Harmonie auf dem Gebiet der Vokalqualität dahin führte. dass das uns bekannte elfgliedrige System sich abzuzeichnen anfing, gab es in der Sprache anscheinend noch nicht alle für die Zusammensetzung dieses Systems erforderlichen Bestandteile. Diese fehlenden Vokale wurden jedoch kraft des S y s te m z w a g s geschaffen, wofür man sich sporadischer oder sonstiger von den allgemeinen Entwicklungslinien abweichenden besonderen Lautveränderungen bediente. Die Frequenz der auf diese Weise entstandenen Vokale war anfangs gering, aber ihre Stellung erstarkte mit der Schöpfung neuer Wörter. Mit Sicherheit berechtigt ist die Behauptung: Wenn die Weiterentwicklung aller vorperm. Vokale sich im Zeichen ausnahmsloser Lautgesetzlichkeit vollzogen hätte. dann hätte das urperm. Vokalsystem sich niemals in der Form herausbilden können, wie es geschehen ist.

Wir wenden unsere Aufmerksamkeit nun der Frage zu, in welchem Ausmass man urperm. Vokale als lautgesetzliche. spontane Fortsetzungen von vorperm. Vokalen betrachten kann und in welchem Ausmass dagegen der Zwang, Bestandteile für den Aufbaı einer Ganzheit zu gewinnen, ihre Entstehung veranlasst hat.

Wie auch im tscher. System können wir am passendsten mit den engen Vokalen beginnen. $i$ ist der Vokal, der sich am besten erhalten hat; es ist eins der wenigen Elemente des zerfallenen vorperm. Systems, das unverändert zum Baustein für das neue System taugte. Verstärkung erhielt es durch das vorperm. ${ }^{*} \bar{i}$, nachdem dieses gekürzt worden war. Der dem $i$ benachbarte Mittelvokal $i$ hat sich durch einen Delabialisierungsprozess sicherlich spontan aus $*_{u}$ und $* \ddot{u}$ entwickelt und wir verstehen auch gut, dass $* \bar{u}$ und sogar 
$*_{\bar{e}}$ (über dieses s. SS. 301-302) zu $\underset{i}{i}$ geworden sind. Hingegen erhebt sich die Frage, ob die Schliessung von vorperm. ${ }^{*} a$ und $*_{o}$ bis zum Aussersten, d.h. zu $u$, nicht dadurch einen Anstoss hat bekommen können, dass das System neben $i$ und $i$ auch einen engen Hintervokal brauchte. LYT'kis hält es für möglich, dass die Schliessung von fi.-ugr. ${ }^{*} a$ und ${ }^{*} o$ im Urpermischen in Verbindung mit einer Ersatzdehnung gewesen sei: als der Vokal der zweiten Silbe apokopiert wurde, dehnte sich der Vokal der Wurzelsilbe und schloss sich zugleich. Nach der allgemeinen Kürzung des langen Vokals wäre aus ihm ein enger kurzer Vokal geworden ('Труды I, SS. 61-64). Obgleich eine sekundäre Vokaldehnung auf permischem Boden in gewissem Masse stattgefunden zu haben scheint (vgl. oben SS. 297, $319-320$ ), ist die Hypothese Lytkins gewiss nicht notwendig gerade zur Erklärung der Schliessung der urspr. offenen Vokale.

Als kleine Abschweifung sei hier die Hypothese von den fi.-ugr. reduzierten Vokalen behandelt, die STEINITz und auf ihn gestützt Lytkin mit den engen Vokalen im Permischen verbinden. Nach Steinitz' Theorie wäre $i$ die Fortsetzung eines hinteren reduzierten Labialvokals $\left({ }^{*} \breve{o}\right), i$ wiederum die eines vorderen reduzierten Labialvokals $\left({ }^{*} \check{o}\right)$ und lllabialvokals $\left({ }^{*} \breve{e}\right)$. Die Entwicklung der Vordervokale hätte sich so vollzogen, dass $*_{\breve{o}}$ durch Delabialisierung mit $*_{\breve{e}}$ zusammenfiel und dieser Vokal dann mit dem fi.-ugr. Vollvokal $*_{i}$ verschmolz (Vok. 125). Lytkin geht noch weiter als Steinitz. Er glaubt nämlich, Beweise dafür zu sehen, dass a lle urperm. engen Vokale, also auch $u$, in Wirklichkeit seinerzeit reduziert gewesen sind (vgl. Древнепермский язык 92-95; Известия Акалемин Наук 1952, S. 481; Труды I, SS. $99-105)$. Es sei kurz festgestellt, dass $\underline{i}$ in einigen wotjakischen Mundarten reduziert worden ist, aber das ist deutlich eine junge Erscheinung; ebenso ist das $\theta$ des PO-Dialektes, das anscheinend ein an das reduzierte $a$ erinnernder Laut ist, nicht ursprünglich, wie Lỵtkin meint, sondern sekundär im Vergleich zum syrj.-wotj. $i$. Dieses, das - wie in diesem Zusammenhang besonders betont sei - nicht bloss kurzes $* u$ und $* \ddot{u}$, sondern auch zwei vorperm. lange Vokale lautgesetzlich vertritt, ist zweifellos gerade in seiner nichtreduzierten Gestalt ein Erbe 
der ältesten perm. Periode. Ein reduziertes $i$ stellt sich auch Steinitz im Urpermischen nicht vor, weil seiner Ansicht nach $*_{\breve{e}}$ und $*_{\check{o}}$ die Qualität des fi.-ugr. "Vollvokals» $i$ angenommen haben. Der Beitrag Lytkins zu dieser Diskussion bedeutet eine Verschlechterung der Theorie von Steinitz. Wie könnte man es für glaubhaft halten, dass $u$, das zur Fortsetzung von * $a$ und $*_{o}$ geworden war, in irgendeinem Stadium des Urpermischen reduziert gewesen wäre? Lytkin begründet seine Auffassung vor allem damit, dass $u, i(=\mathrm{PO} \theta)$ und $i$ zum Unterschied von den übrigen Vokalen der ersten Silbe in mehrsilbigen Wörtern unbetont gewesen sind. Aber diese Tatsache beweist ja nichts für die vorliegende Frage. Die urperm., im Ostpermjakischen immer noch auftretende Akzentuation erklärt sich ganz natürlich als eine Weiterentwicklung des alten - im Finnischen und Lappischen unverändert erhaltenen - anfangsbetonten Akzentuationstypus: Über enge Vokale glitt der Druck in mehrsilbigen Wörtern auf nichterste Silben. Eine ähnliche, wenn auch beschränktere Druckverschiebungserscheinung kennen wir im Urmordwinischen. Der Umstand, dass die engen Vokale hinsichtlich der Akzentuation in eine Sonderstellung geraten sind, rührt ausschliesslich daher, dass sie von viel schwächerer Sonorität sind als die übrigen. Vgl. Vir. 1951, SS. 450-452. Man braucht zur Erklärung der Akzentuationsverhältnisse keine zusätzliche Annahme einer Reduziertheit dieser Vokale und eine solche ist auch nicht mit den sprachlichen Tatsachen zu begründen. Lytkin betrachtet als Hinweis auf eine frühere reduzierte Aussprache ron $u$ und $i$ sogar einen solchen orthographischen Zug, dass diese Vokale in den altsyrjänischen Texten mit den gleichen Buchstaben bezeichnet werden wie die Konsonanten $v$ und j; diese Schreibart muss nach seiner Ansicht als Beweis der aussergewöhnlichen Kürze der beiden Vokale gedeutet werden. Wenn derartige Dinge von irgendwelcher Bedeutung wären, müsste man glauben, dass auch in der finnischen Sprache $u$ und $i$ zur Zeit von Agricola reduziert gewesen seien, ganz zu schweigen von vielen Parallelfällen in der älteren Rechtschreibung der germanischen Sprachen. Auch dem Umstand kann man keinerlei Beweiskraft zugestehen, dass syrj. enge 
Vokale bei der Entlehnung von Wörtern ins Ostjakische oft durch reduzierte Vokale ersetzt worden sind. Nach dem zu urteilen, was PaAsonen, Karjalainen und früher auch Steinitz selbst über die ostj. Vokale geschrieben haben, ist der einzige deutlich reduzierte Vokal in dieser Sprache a, vgl. FUF XXIX 252.

Die zwischen den engen und halbengen Vokalen liegende Vokalreihe $o,{ }^{*} \dot{o},{ }^{*} e$ bildet wegen ihres vom vergleichenden fi.-ugr. Standpunkt gesehen seltenen Artikulationsgrades die merkwürdigste Einzelheit des urperm. Systems. $o$ und $* \dot{0}$ gehören zum organischen Nachwuchs des vorperm. Systems, ersteres als lautgesetzliche Fortsetzung von * $e$ und letzteres von ${ }^{*} \bar{o}$. Die eigenartige Entwicklung des $*_{e}$ zu einem Hintervokal könnte so vor dem Hintergrund der Systemganzheit eine natürliche Erklärung finden: Anfangs wahrscheinlich ${ }^{*} e>{ }^{*} e$, aber als auch das ${ }^{*} \vec{z}$ zu einem Mittelvokal wurde. gab es im System keinen Platz mehr für einen zweiten Mittelvokal von gleichem Artikulationsgrad. Hingegen füllte das $*_{i}$ dadurch, dass es zu o velarisiert wurde, eine Lücke aus, die der Vorgänger des $u$ hinterlassen hatte (frühurperm. ${ }^{*}>u$. und frühurperm. $* e>0$ dürften wie aufeinanderfolgende oder fast parallele Wellen vor sich gegangen sein, was die vielen Fälle erklären würde, in denen die Fortsetzung von vorperm. ${ }^{*} a$ und ${ }^{*} o$ von neuem auf die Form $o$ zurückgeführt worden ist). Der dritte Vokal, ${ }^{*}$, dürfte wohl als lautgesetzliche Fortsetzung von $i$ vor $\check{z}$ angesehen werden. Ein derartiger Lautwandel hätte jedoch kaum stattgefunden, wenn nicht der Systemzwang die Herausbildung des ${ }_{e} e$ erfordert hätte. Dieses vermochte durch sporadische Lautveränderungen seiner äusserst geringen Frequenz einigen Zuwachs aus dem Kreise der benachbarten Vokale zu verschaffen.

Von den halbengen Vokalen ist $o$ hinsichtlich seines Ursprungs klar, es hat sich in ursprünglichen $\ddot{a}$-Stämmen lautgesetzlich aus * $e$ entwickelt. Den entsprechenden Mittelvokal $\dot{o}$ dagegen möchte man am liebsten gänzlich als Ergebnis des urperm. Systemzwangs betrachten. Im alten fi.-ugr. Wortgut erweist es sich in den verhältnismässig seltenen Fällen seines Vorkommens als Fortsetzung von vorperm. ${ }^{*} \ddot{a}$ und ${ }^{*} \ddot{u}$, 
aus denen es sich aber sporadisch entwickelt hat. Der einzige Zug, der eine Art von Regelmässigkeit aufweist, ist der, dass $\dot{o}$ sehr häufig vor $l$ vorkommt. Dafür gibt es etwa 15 Beispiele. Da uns anderseits kein Beispiel für ${ }^{*} \varepsilon$ in entsprechender Stellung bekannt ist, scheint das darauf hinzudeuten, dass vorperm. * $\ddot{a}$ vor $l$ durchweg labialisiert worden ist (hingegen ist ${ }^{*} \ddot{u}$ auch in dieser Stellung ausser zu $\dot{o}$ auch in gewöhnlicher Weise zu $i$ geworden). Der dritte Vokal der halbengen Reihe, $e$, ist ebenfalls im wesentlichen durch abweichende Lautveränderungen bzw. Fälle von Erhaltung entstanden. Zwar scheint ${ }^{*} i>e$ vor $r$ nahezu die Regel gewesen zu sein; ebenso hat vorperm. ${ }^{*} e$ eine starke Neigung gehabt, in der Nachbarschaft eines mouillierten Konsonanten unvelarisiert zu bleiben, aber dennoch ist zuweilen auch bei anderen umgebenden Konsonanten ${ }^{*} i>e$ und ${ }^{*} e \equiv$. Hier dürfte die Erklärung wieder darin zu suchen sein, dass das $e$ seine schwache Frequenz auf dem Wege der sporadischen Lautentwicklung verstärken musste, um als eigene Vokalkategorie in dem System, für das es unentbehrlich war, erhalten zu bleiben.

Die Vokalpaare $o: o, \dot{o}:{ }^{*} \dot{o}$ und $e:{ }^{*} e$ scheinen darauf hinzuweisen, dass im urperm. System das Prinzip des phonologischen Gegensatzes nicht bloss in horizontaler, sondern auch in vertikaler Richtung wirksam war. Wir können z.B. annehmen, dass das unter unklaren Verhältnissen entstandene $\dot{o}$ darin eine Stütze seiner Daseinsberechtigung fand, dass das organisch aus den vorperm. Prämissen gewachsene ${ }^{*} \dot{g}$ eine Entsprechung brauchte. Besonders interessant ist das Paar $e:{ }^{*} \ell$. Das $e$ ist also, wie wir sahen, aus ${ }^{*} i$ vor $r$, das * $e$ wiederum vor $z$ entstanden. Dieses Aufteilungsprinzip, das bei der Entstehung der $e$-Vokale eine wichtige Rolle gespielt hat, ist in merkwürdiger Weise auch im späteren, während der urperm. Zeit geschaffenen Wortgut in Geltung geblieben: Vor $r$ tritt lediglich $e$ auf und entsprechend vor $z$ lediglich *e. Wir kennen eine einzige Ausnahme von dieser Regel, nämlich syrj. t'ser, PO ćir 'Axt' $(<*$ t'śer $)$, wo wir also vor $r$ auf ein *e stossen. Als Gegengewicht haben wir ganze 25 Beispiele für die urperm. Lautverbindung -er-; die Wörter, die diesen Lautkomplex enthalten, bilden übrigens ein Drittel aller $e$-Wörter. (Ein 
zweites Beispiel für die Kontinuität bestimmter Lautkombinationen bietet das häufige Vorkommen von $e$-Vokalen in der Nachbarschaft mouillierter Konsonanten. In dieser Stellung blieben sie ja im Frühurpermischen im allgemeinen von der Velarisierung verschont. Wir haben insgesamt etwa 20 gemeinpermische $e$ - bzw. ${ }^{*} e$-Wörter, in denen unmittelbar vor oder nach dem Vokal ein mouillierter Konsonant steht. Vergleichshalber sei erwähnt, dass ein solcher neben urperm. ${ }^{*} \varepsilon$ in entsprechendem Wortmaterial nur etwa fünfmal aufzutreten scheint und neben $\dot{o}$ kein einziges Mal; neben $i$ wiederum sind mouillierte Konsonanten noch erheblich häufiger als neben $e$-Vokalen.)

Die Vokale der weiten Reihe, $a$ und ${ }^{*} \varepsilon$, haben wir noch nicht betrachtet. Das $a$ ist aus heterogenen Elementen entstanden, in erster Linie wohl unter unklaren Verhältnissen aus * $\ddot{a}$. Es ist recht wahrscheinlich, dass seine Aufnahme unter die Vokale der ersten Silbe durch die Fälle der Erhaltung von vorperm. ${ }^{*} a$ in den nichtersten Silben erleichtert wurde. In dieser Beziehung ähnelt das perm. $a$ dem tscher. $a$. Das ${ }^{*} \varepsilon$ ist eine der vielen Fortsetzungen des vorperm. ${ }^{*} \ddot{a}$, nämlich das Rudiment, das rom $* \ddot{a}$ als weiter Vordervokal übrigblieb, nachdem dieses Elemente von sich abgespalten hatte, um die stellung von 5-6 Vorder-, Mittel- und Hinterrokalen, die eine geringe Frequenz hatten, zu stärken. Einem recht ähnlichen Auflösungsprozess verfiel das ursprüngliche ${ }^{*} \ddot{a}$ auch im T'scheremissischen.

Noch einige Worte über die Entwicklung nach der urperm. Periode. Das gewöhnliche syrj.-wotj. Vokalsystem ist folgendes:

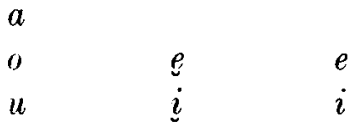

In diesem System hat sich die Zahl der auf der Weite des Artikulationsgrades beruhenden Vokalreihen auf drei verringert, da die zwischen den engen und den halbengen Vokalen stehende Vokalkategorie verschwunden ist. Lautveränderungen, die sich sowohl im Syrjänischen als auch im Wotjakischen 
vollzogen haben, sind folgende: a) $*_{a} \dot{a}>e$, b) ${ }^{*} \underset{x}{ }>e$. Nur im Syrjänischen haben folgende Lautveränderungen stattgefunden: a) $\dot{o}$ (sowohl ursprüngliches wie im Ursyrjänischen aus ${ }_{\varepsilon}$ entstandenes) $>e$, b) $o>0$. Nur im Wotjakischen anzutreffende Lautveränderungen sind folgende: a) ${ }^{*} \varepsilon>e$ (in einem Wiedemaxi bekannten seltenen Dialekttypus gewöhnlich $e$ ). b) $o, o>u$, c) $\dot{a}>o($ vor $l$ meist $a)$, d) $e,{ }^{*} e$ in einem Teil der vorkommenden Fälle $>0$. (Die Entwicklung der Lautverbindungen * ${ }_{-\alpha}$ und * $\boldsymbol{u}_{\alpha} \dot{0}$ übergehen wir an dieser Stelle.)

Abweichend rom allgemeinen Typus haben sich mundartlich einige archaische Züge erhalten. Im Syrjänischen erscheint a) $a$ in einigen altsyrj. Sprachdenkmälern, in der heutigen Sprache im Ober-Sysola-Dialekt und rudimentär auch in den Mundarten von der Unter-Sysola und von Ižma: b) $\ell$ in einigen altsyrj. Texten und in Spuren auch im IžmaDialekt. - Im Wotjakischen scheint sich $\varepsilon$ in begrenztem Umfang erhalten zu haben (in Munkácsis Material).

In einer ganz eigenen Richtung hat sich das ostpermj. Vokalsystem weiterentwickelt, das wir in folgendem Schema darstellen können:

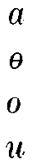

$\begin{array}{ll}\dot{o} & e \\ u & i\end{array}$

Dieses System weist folgende nach der urperm. P'eriode vor sich gegangenen Lautveränderungen auf: a) ${ }^{*} \varepsilon>\dot{o}$ (schon im Ursyrjänischen), b) $0>u$, vor $j u$, c) ${ }^{*} \dot{a}>u$, d) $* \ell>i$, e) $u>$ vor $j$ und mouilliertem Konsonanten $u$, f) $i>\theta$. Als Resultat dieser Veränderungen bildete sich ein interessantes Ganzes von vier Vokidpaaren: $a-\theta, o-u, \dot{o}-u, e-i$. Die Entwicklung des $\theta$ vom engen Mittelvokal zum halboffenen hinteren $a$-Laut hat ein Gegenstück in dem dunklen a mancher heutigen Dialekte des Lappischen. Vom Standpunkt der Systemganzheit aus betrachtet ist diese Entwicklung ja recht natürlich: Nachdem im Ostpermjakischen der Gegensatz $\dot{o}-u$ sich herausgebildet hatte, wäre $i$ als Mittelvokal paarlos geblieben, wenn nicht eine Tendenz sich auszuwirken begonnen hätte, dasselbe dem $a$ anzunähern. 


\section{V \\ SCHLUSSWORT.}

Es dürfte Veranlassung bestehen, einige grundsätzliche Stellungnahmen, zu denen wir in der vorliegenden Untersuchung gelangt sind, zum Schluss noch kurz zu wiederholen, weil sie es verdienen dürften, auch in der künftigen Forschung auf dem Gebiet der finnisch-ugrischen Vokalgeschichte berïcksichtigt zll werden.

Das tscheremissische und permische Material weist keine solchen Vokilverhältnisse auf, zu deren Erklärung man aus der finnisch-ugrischen Ursprache stammende "Ablautwechsel" voraussetzen müsste. Die Auffassung, dass man in beiden Sprachgruppen als Ausgangspunkt der Entwicklıng ein System anzunehmen hat, das dem im Ostseefinnischen anzutreffenden ähnelt, lässt sich nicht nur gut begründen, sondern erscheint überhaupt als die einzig mögliche. So ist eine quantitative Aufteilung, die der finnischen Aufteilung in kurze und lange Vokale entspricht, früher auch in diesen Sprachen vorgekommen, wie wir an Hand qualitativer Tatsachen feststellen können (vgl. besonders SS. 264, 332--333). Solche in früheren Sprachstadien entstandene Parallelformen wie *kolme $\sim{ }^{*} k u l m e$ 'drei' (s. SS. 202-203, 281, 283), *küse *kōse 'Fichte' (SS. 165, 296), *šuje *̌soje 'Spiess, Stachel' (SS. 166, 300), *muślie-

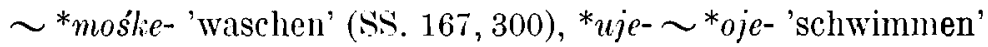
(SS. 169, 218, 287) usw., auf die die Sprachen der finnischpermischen Gruppe hinzudeuten scheinen, erklären sich auf die natürlichste Weise als Ergebnisse von sporadischen Lautveränderungen, nämlich Reihenübergängen zwischen Nachbarvokalen, die nur in einem Teil des Sprachgebiets ror sich gegangen sind. Die ursprüngliche Form hat sich dabei neben der sekundären erhalten und beide haben in den heutigen Sprachen Fortsetzungen. Die interdialektalen lautlichen Inkonsequenzen im Tscheremissischen und die gleichen Inkonsequenzen im permischen Sprachgebiet, die oben auf SS. 243-246 und 316-322 behandelt und in Typen gruppiert worden sind. dürften sich hinsichtlich ihrer Entstehung in keiner Weise von den in verschiedenen Sprachen auftretenden Parallelformen 13 - Finnisch-ugrische Forschungen 
unterscheiden, die soeben erwähnt wurden, sondern als späte Parallelerscheinungen derselben aufzufassen sein.

Sehr gewöhnlich ist es, dass neben der »lautgesetzlichen», d.h. durchweg einheitlich vor sich gegangenen Entwicklung der Elemente des früheren vortscheremissischen bzw. vorpermischen Vokalsystems auch viel lautliche spaltung vorkommt, die oft völlig unregelmässig erscheint, wenn man die Sache rom Standpunkt jenes früheren Systems betrachtet. Das beste Beispiel für eine im wesentlichen ausnahmslose Entwicklung eines bestimmten einzelnen Bestandteiles des Vokalismus der Ursprache bietet sowohl im Tscheremissischen als auch in den permischen Sprachen die Geschichte des urspr. kurzen ${ }^{*} u$. Dagegen weisen urspr. ${ }^{*} a$ und urspr. ${ }^{*} \ddot{a}$ eine starke Spaltung auf; beispielsweise aus $* \ddot{a}$ sind in der tscheremissischen Sprache sogar drei verschiedene Vokale entstanden, die man dennoch alle als normale Vertretungsweisen des *ä betrachten kann. Am verworrensten bleibt das Bild der Geschichte derjenigen vortscheremissischen bzw. vorpermischen Vokale, die nur eine recht geringe Frequenz gehabt haben. Es ist denn auch offensichtlich, dass eine Vokalkategorie um so bessere Voraussetzungen für eine einheitliche Entwicklung besitzt, je grösser ihre Frequenz ist.

Der letztgenannten Beobachtung müssen wir jedoch hinzufügen, dass eine mechanisch regelmässige Entwicklung der einzelnen Vokale lediglich dann erwartungsgemäss ist, wenn im Aufbau des Vokalsystems selbst keine grösseren Veränderungen eintreten. Eine derartige Regelmässigkeit ist solchen Lialutveränderungen eigen, die sich in nur wenig roneinander verschiedenen Mundarten einer Sprache vollziehen. Wenn hingegen im Lauf der Zeiten das Vokalsystem der Ursprache von Grund auf durcheinander kommt und zerbricht, so dass z.B. die Entwicklung der Vokalqualität zu einem erheblich rermehrten Formenreichtum führt - wie es gerade in den von uns untersuchten Sprachen geschehen ist - damn ist vielfache Spaltung unausbleiblich. W a r u m ein Vokalsystem sich in einem späteren Stadium der betreffenden sprache gänzlich verändert, ist eine Frage, die sich mit den Methoden der Sprachwissenschaft im allgemeinen wohl nicht beantworten 
lässt, wenn es sich nicht un eine fremde Einwirkung hanlelt. Eine Tatsache scheint es jedenfalls zu sein, dass dieses in der Herausbildung begriffene neue System die Veränderungen aller einzelnen Vokale lenkt. Also ist die ausnahmslos regelmässige Entwicklung irgendeines ursprünglichen Lautes im Leben der Sprache kein Selbstzweck, wie man es sich früher auch in der finnisch-ugrischen Sprachforschung oft vorstellte, sondern zweckmässig ist nur das, was der Ganzheit, dem System dient. Wenn das neue System seine nötigen Elemente aus dem Sprachmaterial nicht auf dem Wege lautgesetzlicher Entwicklung erhält, so benutzt es als Mittel sporadische Lautveränderungen. Auch die sporadischen Veränderungen jedoch sind gewöhnlich phonetisch nicht granz unmotiviert: Wir sehen, wie die umgebenden Konsonanten durch ihre assimilierende Wirkung den Boden erweitern, den eine neue, gleichsam zufällig entstandene Vokalkategorie in der Sprache hat.

Die Erforschung der finnisch-ugrischen Vokalgeschichte dürfte für einen Forscher, der nicht zugibt, dass ein scheinbar unregelmässiger Lautwandel in Wirklichkeit teleologisch sein kann, zu einer unlösbaren Aufgabe werden. Es wäre jedoch übertrieben, zu behaupten, dass a $11 \mathrm{e}$ unregrelmässigen Veränderungen ron dieser Art seien. Sicherlich sind zufällige Schwankungen vorgekommen, die für die systemganzheit keine Bedeutung gehabt haben; himzu kommen die psychischen Faktoren: das Streben nach Vermeidung ron Homonymie sowie die affektiven, euphemistischen und hypokoristischen Ausdrücke, in denen lautliche Iabilität jeder Art durchaus rewöhnlich ist.

ERkкi Itkonex.

\section{Die von den einzelnen tscher. und ferm. Dialekten gebrauchten Abkürzungen.}

Tscheremis is ch.

TcherO = Osttscheremissisch.

TscherW = Westtscheremissisch.

B $\quad=$ Das Birsker Dialektgebiet im allgemeinen; bei Beke der Dialekt des Dorfes Staro-Orjebaš. 
BC̀ = Der Birsker Dialekt des Dorfes Caškino (tscher. t'śškka) (nach Verfassers Aufzeichnungen vom Jahre 1943).

BČur. = Der Birsker Dialekt des Dorf es Curajeva (nach Paasonen).

BJ $=$ Der Birsker Dialekt des Dorfes Starajaš (tšer. $j a ̈ s$ ) (nach Beke).

BJp = Der Birsker Dialekt des Dorfes joškar-Bamaš (nach Beke).

$\mathrm{C}=$ Das Carevokokšajsker Dialektgebiet im allgemeinen; bei Wichmann der Dialekt des Dorfes Azjal (tscher. $a z-j a \cdot l$ ).

CČ $=$ Der Carevokokšajsker Dialekt des Dorfes Cihajdorova (nach Beke).

GK = Der Carevokokšajsker Dialekt des Dorfes kükš̀nur (nach Beke).

CKr. = Der Carevokokšajsker Diale kt des Dorfes Krasnojar (tscher. $t s a k$ ) (nach Verfassers Aufzeichnungen vom Jahre 1943).

CMU = Der Carevokokšajsker Dialek $\mathbf{t}$ des Dorfes Marij Ušem (nach Verfassers Aufzeichnungen vom Jahre 1942).

CÜ = Der Carevokokšajsker Dialekt des Dorfes üsüt-tür (nach Beke).

C $\quad=$ Das Dialektgebiet von Čeboksar im allgemeinen; bei Beke der Dialekt des Dorfes kuyo-molamas.

$\mathrm{CN}=$ Der Ċeboksarer Dialekt des Dorfes Smincy (tscher. norsola) (nach Beke).

= Das Jaransker Dialektgebiet im allgemeinen; bei Wichmann der Dialekt des Dorfes Lumpanur (tscher. lupà $\cdot n \circ r$ ).

Jo $=$ Der Jaransker Dialekt des Dorfes otâk-sala (nach Beke).

$\mathrm{JP} \quad=$ Der Jaransker Dialekt des Dorfes pokšta (nach Beke).

$\mathrm{JU}=$ Das Jaransk-Uržumer Dialektgebiet im allgemeinen; bei Wichmann der Dialekt des Dorfes Upša (tscher. u.pšo-sola).

JT $=$ Der den Jaransk-Uržumer Dialekttypus vertretende Dialekt des Dorfes Turšomučakš (tscher. turšo-motsaš) (nach Beke).

K = Das Kozmodemjansker Dialektgebiet (nach Aufzeichnungen von Ramstedt, Wichmann, Beke od. Verf.).

M = Das Malmyžer Dialektgebiet im allgemeinen; bei Wichmann der Dialekt des Dorfes Kiljmez (tscher. külmü̈ž), bei Beke der des Dorfes Starinonenger (tscher. tošto-jal).

MK = Der Malmyžer Dialekt des Dorfes Karmankina (tscher. mortal, morkj-jal) (nach Beke).

MM = Der Malmyžer Dialekt des Dorfes Mamakova (tscher. iż-malmâž) (nach Beke).

$\mathrm{P} \quad=$ Das Dialektgebiet von Perm; bei Genetz der Dialekt des Dorfes Nižnij Potam, bei Beke der des Dorfes Sarsi (tscher. sarsade).

$\mathrm{U}=$ Das Uržumer Dialektgebiet im allgemeinen; bei Wichmann der Dialekt der Dörfer Sernur und Kupsola. 
LJ = Der Uržumer Dialekt des Dorfes Serednyj Jadykbyljak (tscher. jajâkplak) (nach Beke).

UP = Der Uržumer Dialekt des Dorfes Petrušin (tscher. pet'sanpotšinga) (nach Beke).

US = Der Uržumer Dialekt des Dorfes Njižnaja süksa (tscher. sü̈kś) (nach Beke).

USj = Der Uržumer Dialekt des Dorfes Sabujal (nach Beke).

$\mathrm{V}=$ Das Dialektgebiet von Vetluga; bei Beke der Dialekt des Dorfes Arba.

S y r jän is c h.

I $\quad=$ Der Ižma-Dialekt.

L $\quad=$ Der Luza-Dialekt.

$\mathrm{P}=$ Das westpermjakische ("komi-permjakische») Dialektgebiet.

Peč. $\quad=$ Der Pečora-Dialekt.

PO = Das ostpermjakische (»komi-jazvinische») Dialektgebiet.

$\mathrm{S} \quad=$ Der Sysola-Dialekt.

SO $\quad=$ Der Ober-Sysola-Dialekt.

$\mathrm{U}=$ Der Udora-Dialekt.

$\mathrm{V} \quad=$ Der Vyčegda-Dialekt.

VL $=$ Der Vyčegda-Dialekt am Flusse Lökčim.

Wo t j a k is c h.

$\mathrm{B}=$ Der Bessermaner Dialekt.

$\mathrm{G}=$ Der Glazover Dialekt.

J $\quad=$ Der Jelabugaer Dialekt.

$\mathrm{II} \quad=$ Der Malmyžer Dialekt.

IUU $=$ Der Malmyž-Uržumer Dialekt.

$\mathrm{U}=$ Der Lifaer Dialekt. 